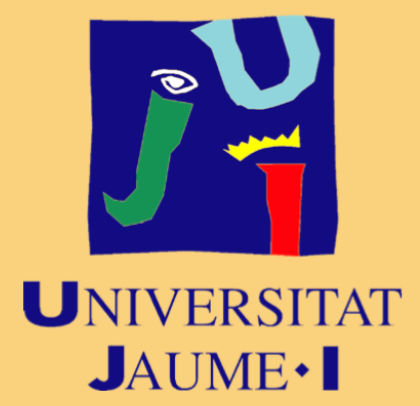

\title{
LA COMPRENSIÓN MORAL DE SITUACIONES ACCIDENTALES Y DELIBERADAS EN ESCOLARES CON AUTISMO
}

\author{
IRENE GARCIA MOLINA \\ Directores:
ROSA ANA CLEMENTE ESTEVAN
CLARA ANDRÉS ROQUETA \\ Directores:
ROSA ANA CLEMENTE ESTEVAN
CLARA ANDRÉS ROQUETA \\ Directores:
ROSA ANA CLEMENTE ESTEVAN
CLARA ANDRÉS ROQUETA \\ IRENE GARCIA MOLINA
}

Castelló, febrer 2019 



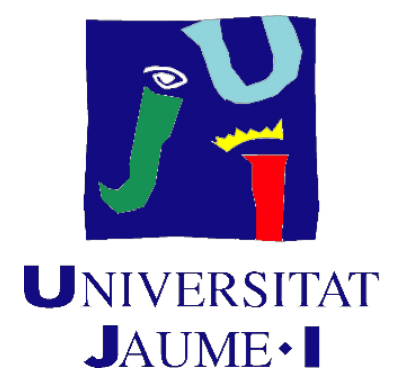

Programa de Doctorat en Psicologia

Escola de Doctorat de la Universitat Jaume I

LA COMPRENSIÓN MORAL DE SITUACIONES ACCIDENTALES Y DELIBERADAS EN ESCOLARES CON AUTISMO

LA COMPRENSIÓ MORAL DE SITUACIONS ACCIDENTALS I DELIBERADES EN ESCOLARS AMB AUTISME

MORAL UNDERSTANDING OF ACCIDENTAL AND DELIBERATE SITUATIONS IN AUTISTIC CHILDREN

Memòria presentada per IRENE GARCIA MOLINA per a optar al grau de doctora per la Universitat Jaume I

IRENE GARCIA MOLINA

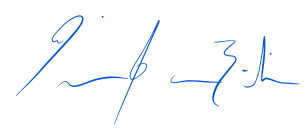

Castelló de la Plana, febrer de 2019.
ROSA ANA CLEMENTE ESTEVAN

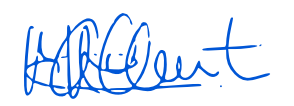

CLARA ANDRÉS ROQUETA

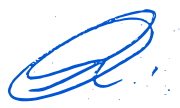




\section{Finançament rebut}

Aquesta tesi doctoral ha sigut possible gràcies al finançament rebut per la Generalitat Valenciana, Programa VALi+D per a investigadors en formació, de la que jo, Irene Garcia Molina, vaig poder gaudir del 1/7/2015 al 30/6/2018.

A més, l'any 2016 i l'any 2017, la Generalitat Valenciana, a través del programa de beques per a estades de contractats predoctorals en centres d'investigació fora de la Comunitat Valenciana, m'atorgà poder realitzar dues estades en el Centre for Research in Autistm and Education (CRAE) de l'Institute of Education, dintre de la University College London (UCL). Aquestes estades duraren un total de 6 mesos, la primera de 3 mesos (del 2/9/2016 al 14/12/2016) i la segona de 3 mesos més (del 8/6/2017 al 9/9/2017).

\section{Financiación recibida}

Esta tesis ha sido posible gracias a la financiación recibida por la Generalitat Valenciana, Programa VALi+D para investigadores en formación, de la que yo, Irene Garcia Molina, pude disfrutar del 1/7/2015 al 30/6/2018.

Además, el año 2016 y el año 2017, la Generalitat Valenciana, a través del programa de becas para estancias de contratados predoctorales en centros de investigación fuera de la Comunidad Valenciana, me otorgó poder realizar dos estancias en el Centre for Research in Autism and Education (CRAE) del Institute of Education, dentro de la University College London (UCL). Estas estancias duraron un total de 6 meses, la primera de 3 meses (del 2/9/2016 al 14/12/2016) y la segunda de 3 meses más (del 8/6/2017 al 9/9/2017). 
"We're told in school we've got five senses. Wrong! We've got six. The sixth one being our theory of mind; the ability to understand everybody else's thought processes. So, if you have the ability to do that, don't please, don't ever say you could be a little bit autistic."

Carly Jones, an autistic woman.

‘Things not to say', in BBC Three (21st of June, 2016). 


\section{Agradecimientos / Agraïments / Acknowledgements}

Als meus pares i a les meves amigues i amics (en especial a Judith, Ismael, Marta i Raquel), per la paciència i els ànims.

A les meves directores de tesi, Rosana i Clara, per encaminar-me en tot aquest treball.

I thank London and CRAE for having me during months and teaching me every day (especially to Liz Pellicano, Anna Remington, Melissa Bovis, Laura Crane, Ellie Buckley, Eilish Roy, Becca Sealy, Jana Brinkert, Lorcan Kenny, Eleanor Palser, and also to Dominique Girard).

Als mestres, xiquetes i xiquets dels centres que han participat en fer possible aquesta tesi (IES Bovalar, CEIP Gaetà Huguet, CEIP Penyagolosa, CEE Pla d'Hortolans, Psicotrade, CEIP Recaredo i CEIP Tombatossals).

Finalment, i especialment:

Al meu germà, per ser un referent per a mi, en el món de la investigació i fora d'ell.

A José Luis, per moure mar, cel i muntanyes, i aconseguir fer aquest camí el més planer possible. 


\section{ÍNDICE / TABLE OF CONTENTS}

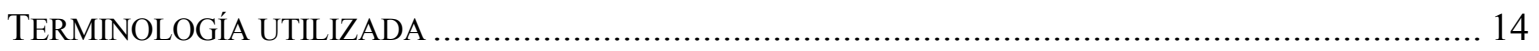

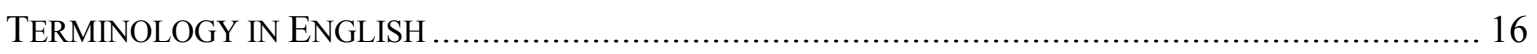

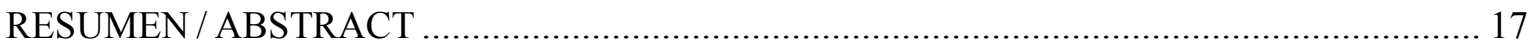

CAPÍTULO 1 INTRODUCCIÓN Y JUSTIFICACIÓN......................................................... 21

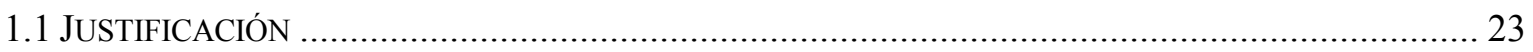

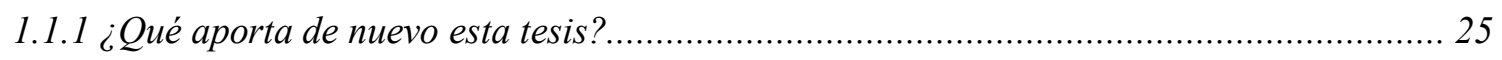

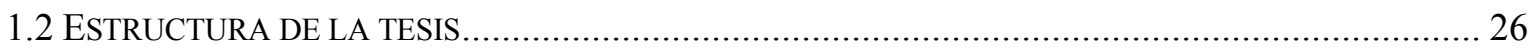

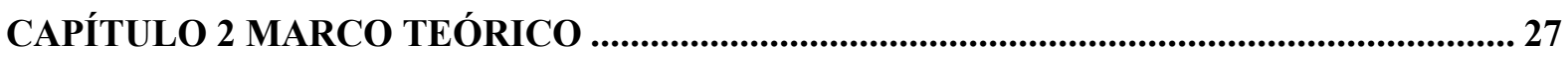

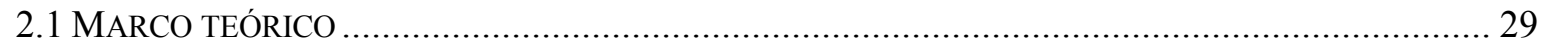

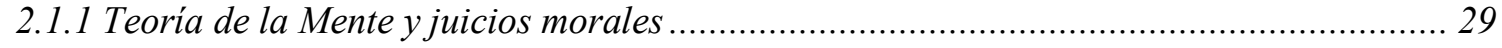

2.1.2 El peso de los estados mentales en los razonamientos morales ...................................... 35

2.1.3 El peso de los estados mentales en los razonamientos morales de las personas con TEA 42

2.1.4 Los juicios morales en las situaciones accidentales ...................................................... 44

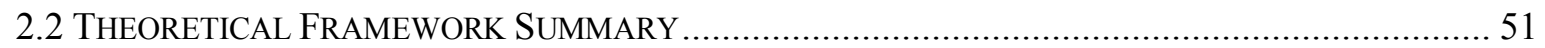

CAPÍTULO 3 OBJETIVOS DE LA INVESTIGACIÓN Y METODOLOGÍA........................... 53

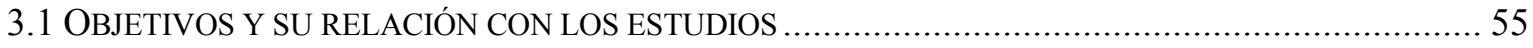

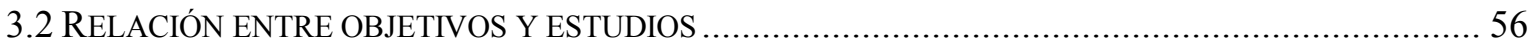

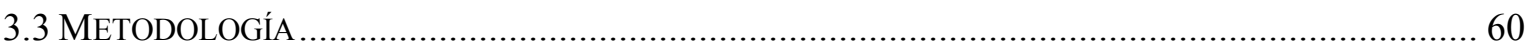

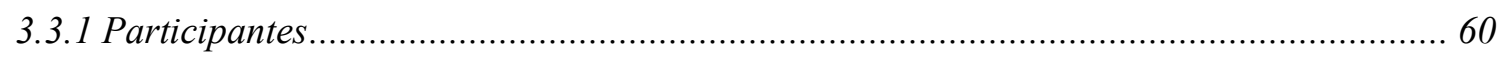

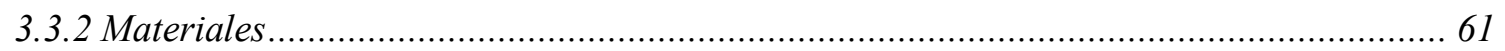

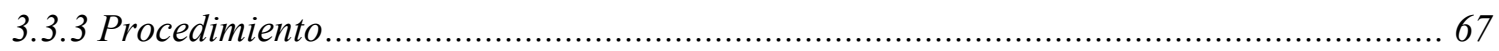

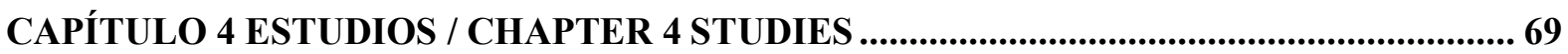

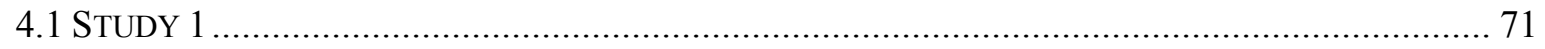

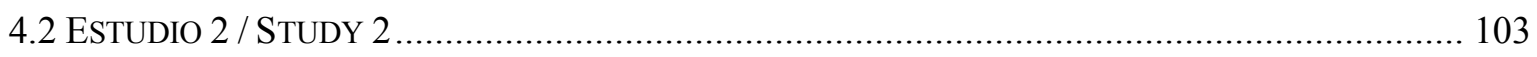

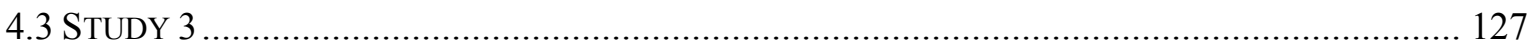

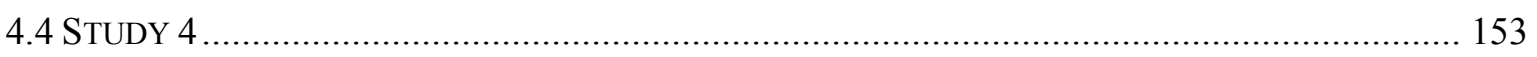

CAPÍTULO 5 / CHAPTER 5 GENERAL DISCUSSION AND CONCLUSIONS ..................... 177

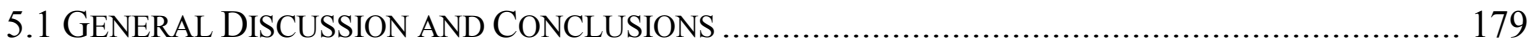

5.1.1 Discuss the differences between groups in deliberate and accidental situations. ........... 180 
5.1.2 Recognise whether certain factors influence the understanding of a faux pas. 189

5.1.3 Analyse the differences between Moral Transgressions and Faux Pas tasks for both autistic and NT participants (Study 4). 194

5.2 GENERAL DISCUSSIONS AND CONCLUSIONS SUMMARY .................................................... 196

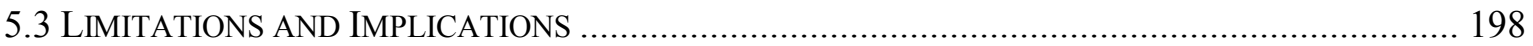

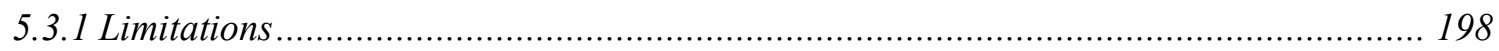

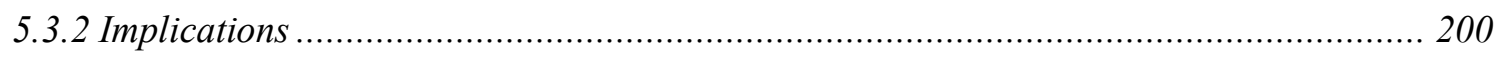

CAPÍTULO 6 REFERENCIAS / CHAPTER 6 REFERENCES........................................... 201

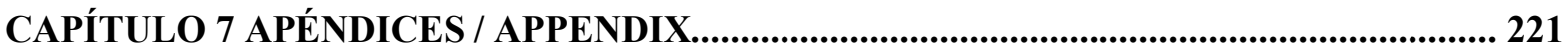

7.0 APÉNDICE 0. PERMISOS PARA LA ADMINISTRACIÓN DE LAS TAREAS (TEA Y NT) .................. 223

7.1 APÉNDICE 1. HiSTORIAS VISUALES DE METEDURA DE PATA / ViSUAL FAUX PAS STORIES...... 225

7.2 APÉNDICE 2. HiSTORIAS VERBALES DE METEDURA DE PATA / VERBAL FAUX PAS STORIES.... 226

7.3 APÉNDICE 3. HISTORIAS MIXTAS DE METEDURA DE PATA / MIXED FAUX PAS STORIES ........... 228

7.4 APÉNDICE 4. HISTORIAS VERBALES MORALES / VERBAL MORAL STORIES............................... 231

7.5 APÉNDICE 5. HISTORIAS MIXTAS MORALES / MIXED MORAL STORIES ..................................... 232

7.6 APÉNDice 6. Historia FALSA CREENCIA NO VERBAL / NON-VERBAL FALSE BELIEF STORY. 235 


\section{ÍNDICE DE TABLAS / LIST OF TABLES}

\section{Tablas Capítulo 3}

Tabla 3-1 Datos descriptivos de los grupos por estudio 61

Tabla 3-2 Tareas administradas en cada estudio 67

\section{Tablas Capítulo 4}

Table 4-1 Mann-Whitney U scores by question and groups in the moral task $(\min =0 ;$ max $=3) \ldots 90$

Table 4-2 Mean and Mode (the level of the category) in ASD and NT group (min =0; $\max =4)$.... 92

Table 4-3 Morality categorisation and real examples of justifications ......................................... 94

Table 4-4 Intention categorisation and real examples of justifications......................................... 95

Tabla 4-5 Coeficientes tipificados y valores de probabilidad ................................................... 119

Tabla 4-6 Medias y DT de las respuestas dicotómicas de elección y Suma Total ......................... 119

Table 4-7 Participants' Descriptive Data, Using Student's $t$ Statistic ....................................... 138

Table 4-8 Story Classification by Modality and Error ....................................................... 140

Table 4-9 Between-groups Differences on Faux Pas Test According to Modality (t test for

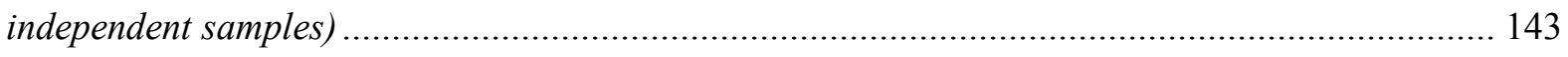

Table 4-10 Differences between Modalities, by Group (Paired t Test) ..................................... 144

Table 4-11 Bivariate Correlations between Faux Pas, Visual WM, Verbal WM, and IQ in the ASD and NT Groups.

Table 4-12 Summary of the Regression Analysis and Step-wise Procedure to Predict Faux Pas Test Results in Different Modalities (Visual, Verbal, and Mixed), in the ASD Group Error! Bookmark not defined.

Table 4-13 Decoding features involved in MT and FP stories 165

Table 4-14 Differences between groups (t-test) divided by personal or material mediators 169

\section{ÍNDICE DE CAJAS / LIST OF BOXES}




\section{ÍNDICE DE FIGURAS / LIST OF FIGURES}

Figura 2-1. Modelo sobre cómo se forma la intención, extraído de Tomasello et al. (2005). 36

Figura 2-2. Descomposición de una acción intencional basada en la transgresión del veneno (Cushman, 2015).

Figura 2-3. Situaciones que surgen de la combinación de los factores: intención, resultado y acción (de Cushman, 2015; Martin y Cushman, 2016; y Young, Cushman, Hauser y Saxe, 2007).............. 41

Figura 2-4. Comparación TEA - NT en los elementos que repercuten en una transgresión.............. 44

Figura 2-5. Descomposición real de una metedura de pata............................................................. 46

Figura 2-6. Estructura de las dos vías (diferentes perspectivas) en una metedura de pata. ................ 48

Figura 3-1(a). Construcción de los cuatro estudios según los objetivos planteados............................ 57

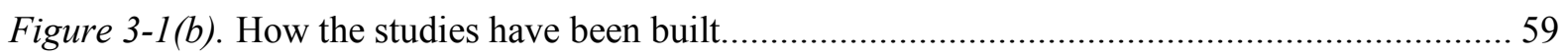

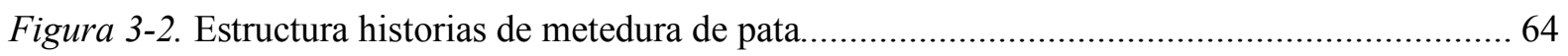

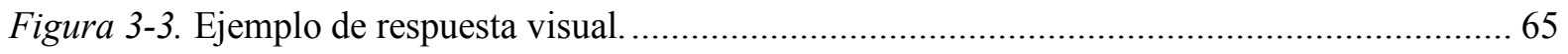

Figura 3-4 Estructura historias de transgresión moral. .................................................................. 66

Figure 4-1. Number of justifications put forward by participants with ASD versus NT, classified into categories (i), (ii), (iii) or (iv), in response to action-morality questions on the (a) Dog, (b) Car, and (c) Sandwich story. 92

Figure 4-2. Number of justifications put forward by participants with ASD versus NT, classified into categories (i), (ii), (iii) or (iv), in response to intention questions on the (a) Dog, (b) Car, and (c) Sandwich story.....

Figure 1-3. Number of justifications put forward by participants with ASD versus NT, classified into categories (i) ToM content and (ii) No-ToM content in response to action-morality questions on (a) Dog, (b) Car, and (c) Sandwich story. 96

Figure 1-4. Number of justifications put forward by participants with ASD versus NT, classified into categories (i) ToM content and (ii) No-ToM content in response to intention questions on (a) Dog, (b) Car, and (c) Sandwich story.

Figura 4-5. Número de niños por grupo y sus puntuaciones en explicación en las historias de metedura de pata.

Figure 4-6. Intention of the action broken down into its elemental features and morality (modified from Cushman, 2015).

Figures 4-7 and 4-8. Mean scores of the NT (2) and ASD (3) groups according to their judgements on: intention, agent, action and outcome in MT and FP tasks. See scores in section b) of Material: Scores on the ToM task (maximum of 4 on badness / deliberate). 


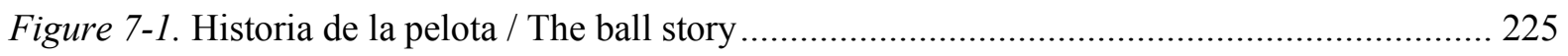

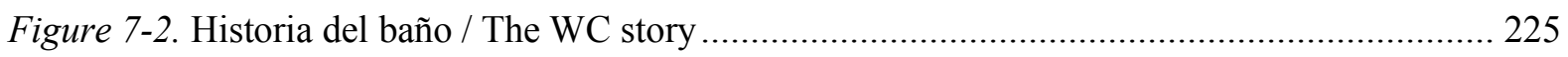

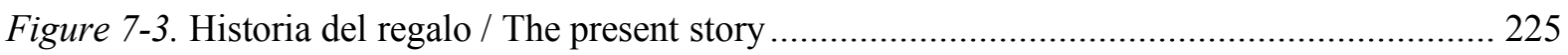

Figure 7-4. Historia del pastel de manzana / Apple pie story........................................................ 228

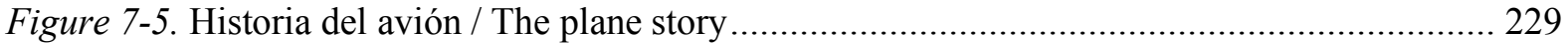

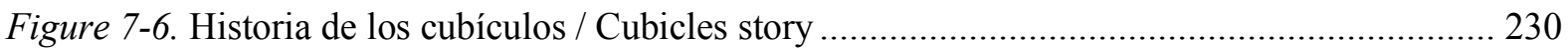

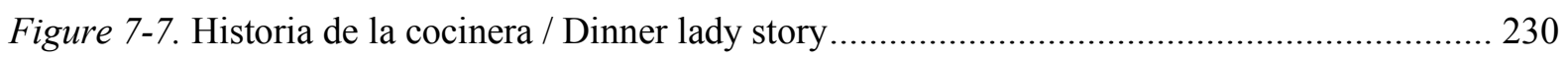

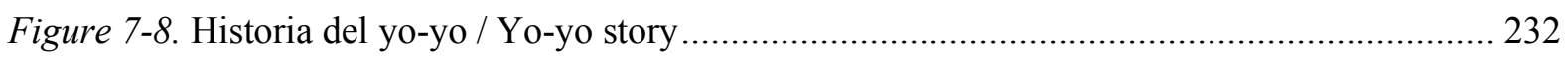

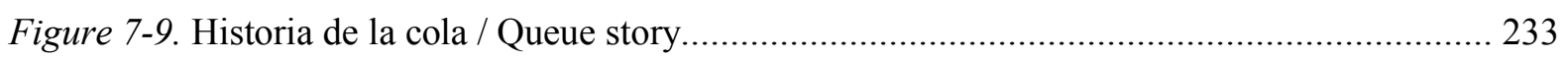

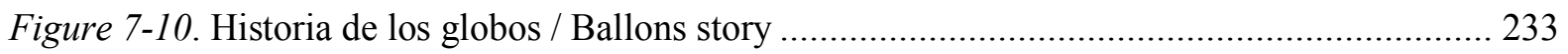

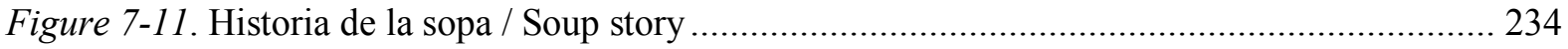

Figure 7-12. Historia de la falsa creencia no-verbal / Non-verbal false belief story ..................... 235

\section{LISTA DE ABREVIACIONES / LIST OF ACRONYMS}

\section{Castellano}

- CI: Cociente Intelectual

- FC: Falsa Creencia

- NT: Neurotípico/a

- TEA: Trastorno del Espectro Autista

- TM: Teoría de la Mente

\section{English}

- ASC: Autism Spectrum Condition

- ASD: Autism Spectrum Disorder

- FB: False Belief

- FP: Faux Pas

- IQ: Intelligence Quotient

- MT: Moral Transgression

- NT: Neurotypical

- ToM: Theory of Mind

- WCC: Weak Central Coherence

- WM: Working Memory 


\section{Terminología utilizada}

\section{Esta tesis respeta las preferencias terminológicas acerca del autismo.}

El lenguaje que se utiliza en referencia a la diversidad funcional, y más en concreto en este trabajo para describir el autismo, repercute en nuestras percepciones sobre dicha condición y, a su vez, es capaz de moldear un deseo común de lucha por los derechos de estas personas (Garcia-Molina, in press).

Por esta razón, Kenny, Hattersley, Molins, Buckley, Povey y Pellicano (2016), realizaron una encuesta en Reino Unido sobre las preferencias en el uso del lenguaje relacionado con el autismo. En base a los resultados de dicha encuesta, se crearon una serie de pautas sobre los términos más aceptados, con el fin de que las personas interesadas en escribir sobre autismo pudieran hacerlo de una forma adecuada.

Si bien estas pautas son flexibles, esta tesis es sensible a las preferencias expresadas por la comunidad autista del Reino Unido en dicho estudio de 2016, así como aquellas expresadas por la población de habla española en Garcia-Molina (in press). Aun así, se tiene que tener en cuenta de que éste se trata de un contexto académico, y tal y como muchos encuestados refirieron en sus respuestas, depende del contexto en el que se esté hablando o escribiendo, se deben seguir unas normas más académicas. Por tal razón, se nombrará como diagnóstico el término Trastorno del Espectro Autista, siguiendo el DSM-5 (APA, 2013), en la descripción de la población, o su acrónimo TEA en la comparación intergrupal (grupo TEA) y también en gráficas, tablas o figuras.

A continuación, se encuentra un resumen del uso de dicha terminología según las preferencias expresadas en la población inglesa e hispana. Siguiendo el formato de la guía terminológica de la revista Autism, se detalla la terminología utilizada en inglés también (Terminology in English). 


\section{Preferencias}

Para las personas con autismo:

- autistic person es el término exacto en inglés, pero debido a que la forma exacta en castellano sería Nombre + Adjetivo (ver la sintagmación de las lenguas romance en Luna, 2015) en la presente tesis se usará persona con autismo.

- Autismo

- En el espectro autista

- Tiene: autismo / Trastorno del Espectro Autista (TEA) / condición del espectro autista Para los grupos de comparación:

- Neurotípico

- Niños o adultos con desarrollo típico*

- Grupo de comparación

*Se usará el término 'neurotípico', y su acrónimo NT. Cuando en los estudios aparezca la abreviatura DT se referirá a la Desviación Típica (DT).

\section{Evitar el uso de...}

Para los individuos con autismo, se debe evitar:

- Sufre, padece, o es víctima del autismo

- Autismo de Kanner

- Autismo como enfermedad

- Autismo de bajo o alto funcionamiento (en lugar de esto, hacer hincapié en la descripción más precisa de las habilidades de dicha persona).

Para los grupos de comparación en investigaciones, se debe evitar:

- Normal, desarrollo normal, o sano.

- Grupo control. 


\section{Terminology in English}

\section{This thesis respects the preferences of autistic community}

\section{Preferred language}

The term 'autistic person / people' was the most commonly preferred term for autistic adults in Kenny et al. (2016). The most preferred term amongst all stakeholders, on average, was 'people on the autism spectrum'.

\section{Non-preferred language:}

- Suffers from or is a victim of autism. Consider using the following terms instead:

- is autistic

- is on the autism spectrum

- has autism / an autism spectrum disorder (ASD) / an autism spectrum condition (ASC)

- Kanner's autism

- Referring to autism as a disease / illness. Consider using the following instead:

- autism is a disability or autism is a condition

- Retarded / mentally handicapped / backward. Consider using the terms intellectual disability or developmental delay instead.

- Referring to Asperger's syndrome as a rare / mild form of autism. Just because someone appears to be verbally or cognitively able, does not mean they cannot be severely affected by their autism.

- Low or High Functioning Autism. A more precise description of people's abilities is encouraged (such as referring to their cognitive or verbal abilities).

Related to comparison groups:

- Avoiding the term normal or normally developing and healthy. Consider using the following terms instead:

- Neurotypical

- Typical adults or typically developing children

As a more general point, Kenny et al. (2016) and the journal Autism also advocate referring to comparison rather than control groups, as it is almost always unclear what is being controlled for. 


\section{RESUMEN / ABSTRACT}

\section{Resumen}

Esta tesis presenta una serie de evidencias teóricas y empíricas para comprender la interpretación que realizan los escolares con un diagnóstico de Trastorno del Espectro Autista sobre la moralidad utilizando situaciones en las que se manipula la intencionalidad y se contemplan los resultados a la luz de la Teoría de la Mente (TM). Tras una breve sección de justificación (Capítulo 1), se formulan y describen los antecedentes teóricos y literarios más relevantes sobre moralidad y autismo (Capítulo 2) ${ }^{1}$, estableciendo, de esta forma, la línea directriz que ha impulsado los trabajos empíricos derivados. Después del capítulo metodológico (Capítulo 3), en el que se describen los objetivos globales del trabajo, se accede al Capítulo 4, dónde se hallan los cuatro estudios concretos. Los Estudios 1, 3 y 4 (estudio escritos en inglés) comparan escolares con autismo y neurotípicos. El Estudio $2^{1}$ (estudio escrito en castellano) compara dos grupos de niños con autismo con diferente nivel intelectual. En relación con los objetivos de cada uno de los trabajos, todos ellos siguen una línea de continuidad que se basa en el análisis de los primeros resultados para construir los siguientes estudios. Por ejemplo, las conclusiones del primero y segundo estudio (peso verbal, memoria de trabajo, estados mentales y forma de procesar la información) se analizaron para construir el tercer y cuarto estudio. Así pues, en el Estudio 1 se comparan las respuestas de dos grupos de niños con y sin autismo ante una nueva tarea moral que se presenta en formato verbal. El Estudio 2 analiza las variables implicadas en la comprensión de las meteduras de pata (situaciones accidentales) en el autismo (como el Cociente Intelectual; CI o la Falsa Creencia; FC) y examina las dificultades que los niños con autismo han podido encontrar. Basados en las conclusiones del Estudio 1 y 2, acerca del peso verbal de la tarea y las posibles influencias del CI, Memoria de trabajo y estados mentales, el Estudio 3 (estudio en inglés), trata de discernir qué modalidad de presentación (visual, verbal o mixta) puede ser la más adecuada para que los escolares con autismo comprendan mejor una metedura de pata, y las posibles influencias de otros factores en su resolución (como la Memoria de Trabajo). En el Estudio 4 (estudio en inglés), se establece la posible relación entre intención y moralidad. También, se analizan las sutiles dificultades que los niños con autismo de este trabajo pueden encontrar en situaciones

\footnotetext{
${ }^{1}$ Tanto el Marco Teórico como el Estudio 2 tienen un resumen largo en inglés para que lectores ingleses puedan comprender lo que en dicha sección o estudio se detalla.
} 
accidentales y deliberadas debido a sus problemas en TM. Para finalizar, en el Capítulo 5 (Chapter 5. General Discussion and Conclusions) se encuentran la discusión general y las conclusiones que se centran en los objetivos de la tesis y remarcan las diferencias encontradas, las posibles influencias de otros factores en la resolución de tareas de TM avanzada y la importancia de los estados mentales en el razonamiento moral, así como las limitaciones e implicaciones posibles en la vida diaria de las personas con autismo. Debido a que la presente tesis opta a la mención de doctorado internacional, dicha parte está escrita completamente en inglés.

\begin{abstract}
This thesis presents some theoretical and empirical evidence to help understand the interpretations that children diagnosed with Autism Spectrum Disorder make about morality. The responses to the scenarios, involving accidental and deliberate situations, are analysed based on the concept of Theory of the Mind (ToM). After a brief section outlining the rationale (Chapter 1), the most relevant theoretical antecedents of morality and autism are described (Chapter 2) ${ }^{2}$, in order to establish the guidelines that have driven the derived empirical works. The methodology is then presented in Chapter 3, in which the aims of the thesis are set out. Chapter 4 contains the four studies, which are the most relevant part of the present research. Studies 1, 3 and 4 (written in English) compare autistic children to neurotypical children. Study 2 (written in Spanish) compares a group of autistic children with low average IQ to autistic children with high average IQ. Regarding the objectives of each study, all of them follow a continuous thread based on the previous findings. The results from one study lead on to the next. For example, the conclusions of Studies 1 and 2 (the possible influences of verbal weight, working memory, mental states and information processing) were analysed to shape Studies 3 and 4. Therefore, in Study 1, a comparison of responses to a new verbal moral task between two groups of children with and without autism is explored. Study 2 analyses the variables involved in the understanding of faux pas (accidental situations) in autism (such as the Intelligence Quotient - IQ - or the False Belief - FB) and examines the difficulties that autistic children can encounter in such situations. Based on the conclusions of Studies 1 and 2 about verbal weight and the effect of IQ, working memory and mental states, Study 3 tries to
\end{abstract}

\footnotetext{
2 Both the Theoretical Framework and the Study 2 have a long summary in English so that English-speaking readers can understand what is detailed in that section or study.
} 
distinguish what modality (visual, verbal or mixed) is the most adequate for autistic children when accidental actions are presented. It is also argued that some variables can affect the autistic children's answers (i.e., working memory). In Study 4, the possible relationship between intention and morality is established. The subtle difficulties of autistic children in accidental and deliberate situations due to their problems with ToM are discussed. Finally, in Chapter 5, we find the General Discussion and Conclusions, which focus on the objectives of the thesis and highlight the differences found, the possible influences of other factors in the resolution of advanced ToM tasks and the importance of mental states in moral reasoning. Furthermore, some limitations and possible implications for the daily life of autistic individuals are also discussed. Due to the fact that this thesis opts for the international doctorate distinction, this last part is written entirely in English. 
Capítulo 1 Introducción y Justificación 


\subsection{Justificación}

Según la última estimación, se ha conocido una prevalencia del Trastorno del Espectro Autista (TEA) del 1.7 por ciento, es decir, de 1 de cada 59 nacimientos en Estados Unidos (Centro para el Control y la Prevención de Enfermedades; CDC, 2018), y de 1 de cada 100 nacimientos en Europa (Autism-Europe, 2018). El crecimiento casi exponencial en estos últimos años ha tenido consecuencias en el campo de la investigación, tratando de dar respuesta a preguntas e hipótesis centradas en entender cómo procesan la información las personas con autismo, a qué se deben las dificultades sociales que suelen encontrar y cómo repercutir en una mejora de su calidad de vida. Esta indagación se ha puesto de manifiesto, mayoritariamente, los últimos años, gracias al modelo de inclusión y normalización educativa en nuestro sistema escolar, por el cual las personas con autismo - sobre todo aquellas sin una afectación cognitiva notable - pueden tener acceso a la Educación Secundaria, e incluso Superior. Debido a dicha inclusión en contextos donde existen personas con y sin autismo, es común que las dificultades que definen dicho trastorno, sobresalgan (ver DSM-5; APA 2013). Como es ampliamente conocido, los problemas más comunes que los individuos con dicha condición pueden encontrar se relacionan, de forma relevante, con el ámbito social y de la comunicación: los problemas para iniciar o responder a interacciones sociales, las limitaciones en interpretar los estados mentales ajenos durante las interacciones, o las dificultades para ajustar su comportamiento con el fin de encajar en diferentes contextos, son algunos de los ejemplos que pueden incidir de manera más diferencial en su día a día. Esta realidad, supone un reto para la figura del investigador, psicólogo o educador, acerca de la necesidad creciente de proporcionar a las personas con TEA una adecuada intervención en aspectos comunicativos y sociales para su generalización en la vida real.

La habilidad para comprender los estados mentales ajenos en nuestras situaciones sociales, cómo actúan los otros (en presente, pasado y futuro) o incluso cómo hacer amigos, es conocida como Teoría de la Mente (TM; ver Marco Teórico para incidir sobre el tema). A través de la cual, podemos interpretar y comprender los estados mentales de los demás, sus conocimientos, creencias y deseos, y actuar en consecuencia (Frith y Frith, 2005). Dicha TM, como todo proceso madurativo, contempla varios estadios o niveles que se alcanzan progresivamente con la edad y las experiencias sociales (Astington, 2001; Wellman y Lagatuta, 2000; Wellman y Liu, 2004). Sin embargo, en referencia a las tareas de evaluación de la TM, existe un amplio abanico de tareas mentalistas que se centran, de forma muy preferente, en los 
primeros niveles de adquisición, destinadas a niñas y niños pequeños. Hasta donde se conoce a día de hoy, existen muchas menos tareas centradas en habilidades mentalistas que exijan una capacidad más madura, habitualmente desarrollada en la etapa pre-adolescente o adolescente, y que, además, se relacionen con situaciones cercanas a la realidad y comunes en la interacción diaria. Por esta razón, la presente tesis doctoral, al mismo tiempo que adopta un formato clásico de investigación y conocimiento, aporta un plus en su contenido, debido a que las tareas de TM avanzada que en ella se utilizan, han sido creadas por el equipo y publicadas en Garcia-Molina, Clemente, Andrés-Roqueta y Rodríguez (2016).

Estas tareas contempladas en el presente trabajo y los diferentes artículos, constan de breves historias que ilustran y simulan situaciones que suelen ocurrir en diferentes contextos de interacción social, con altas implicaciones emocionales, morales y de intencionalidad. Dichas implicaciones, son cruciales en la comprensión de situaciones más morales, como reconocer a posibles amigos o enemigos, juzgar los comportamientos ajenos o percibir si un grupo de personas nos conviene o no para cualquier relación. Por ejemplo, no se juzga de la misma forma a una persona que hace daño deliberadamente a otra, que a una persona que realiza el mismo daño de forma accidental. Pues bien, en esta distinción, se debería considerar la intención de las personas, y para reconocer dicha intención, se debe atribuir y reconocer los estados mentales de los demás, y es en esto, como se ha referido en párrafos anteriores, en lo que encuentran dificultades las personas con autismo.

Dicha problemática, puede resultar más o menos complicada en las tareas psicológicas, sin embargo, el problema se encuentra en el día a día de las personas con autismo. En su vida cotidiana, las personas con autismo pueden sufrir casos de bullying, difícilmente detectables por ellos mismos o sus familiares, o ser consideraros un mate crime, un 'amigo' del que aprovecharse por su dificultad en interpretar las intenciones ajenas (Grundy, 2011; Thomas, 2011).

En esta misma línea, las complicaciones para entender los estados mentales ajenos pueden repercutir en situaciones más habituales, como las meteduras de pata, consideradas más difíciles de detectar por su alto nivel de comprensión mentalista, así como las valencias ambiguas entre intención y resultado: intención neutra-buena / resultado malo (ver Margoni y Surian, 2016). Este hecho, suele repercutir en malinterpretar la situación y atribuir intencionalidad a un accidente, por lo que implicaría también un malentendido moral con posibles repercusiones en las relaciones diarias (ver estudios de Moran et al., 2011; Zalla, Sav, Stopin, Ahade y Leboyer, 2009). 


\subsection{1 ¿Qué aporta de nuevo esta tesis?}

Tal y como se ha expuesto, es de vital importancia el uso de nuevas historias, más ecológicas y comunes, que contemplen aspectos mentalistas, sobre intenciones y moralidad en la misma tarea, con el fin de no recurrir a varias medidas independientes que midan varios aspectos, o a las tareas prototípicas de TM extensamente administradas, en su mayoría sobre creencias falsas, con poco contenido social y con dudoso aporte mentalista ligado a la realidad (Killen, Mulvey, Richardson, Jampol y Woodward, 2011). De hecho, son escasos los estudios que han utilizado medidas similares a las administradas en la presente tesis, y que, además, han comparado situaciones sociales del nivel de las meteduras de pata (acción accidental) con transgresiones morales (acción deliberada) en población con autismo de habla hispana, aportando reflexiones acerca de la moralidad que se pueden aplicar constantemente a nuestros razonamientos mentalistas habituales.

Por todo ello, la investigación que se muestra pretende ser útil en su literatura, aportes teóricos, novedades y discusiones a nivel mentalista y moral, tratando de dar respuesta a las dificultades - muchas veces sutiles y que pasan desapercibidas en el contexto social - que las personas con autismo suelen encontrar en su día a día en referencia a la intencionalidad y moralidad. Si, por un lado, las personas con autismo, malinterpretan la intención benévola del otro entendiéndola como negativa, esto podría repercutir en las relaciones con sus pares en el sentido de perder oportunidades para hacer amigos o conservarlos. Por otro lado, si malinterpretan una intención negativa como buena, esto podría ser peligroso para ellos, llegando a ser vulnerables como mate crimes o ante situaciones de bullying.

Así pues, la investigación de esta tesis pretende ser un primer paso que se espera pueda tener un efecto colateral positivo en la calidad de vida de las personas con autismo. 


\subsection{Estructura de la tesis}

A continuación, se expone resumidamente la estructura de la tesis.

En sí, la tesis está organizada por siete capítulos, entre los que se encuentran: Capítulo 1. Introducción y justificación; Capítulo 2. Marco teórico; Capítulo 3. Objetivos de la investigación y Método; Capítulo 4. Estudios; Capítulo 5. General Discussion and Conclusion; Capítulo 6. Referencias; y Capítulo 7. Apéndices.

Todos los estudios tendrán una estructura similar. Primero se dará una visión general del tema del artículo y se incidirá en los hallazgos acerca de los individuos con autismo, para finalmente detallar las conclusiones sobre el tema.

La información que se encuentra en esta tesis, además, ha sido enviada o se encuentra aceptada para la publicación en diversas revistas. A continuación, se detallan todas ellas y se especifica el estado del manuscrito.

Estudio sobre Terminología. Garcia-Molina, I. (EN PRENSA/IN PRESS). Preferencias terminológicas acerca del autismo según participantes de un MOOC sobre inclusión educativa. Psicología, Conocimiento y Sociedad.

Revisión del Marco Teórico. Garcia-Molina, I. \& Clemente-Estevan, R. (EN PRENSA/IN PRESS). El razonamiento moral en personas con Trastorno del Espectro Autista: una revisión teórica. Estudios de Psicología.

Estudio 1. Garcia-Molina, I., Clemente-Estevan, R.A. \& Andrés-Roqueta, C. (EN REVISIÓN/UNDER REVIEW). Moral Judgements in Autism. A novel moral judgement task. Cognitive Development.

Estudio 2. Garcia-Molina, I., \& Clemente-Estevan, R.A. (EN REVISIÓN/UNDER REVIEW). La comprensión de las meteduras de pata en escolares con autismo. Su relación con variables cognitivas y de teoría de la mente. Universitas Psychologicas.

Estudio 3. Garcia-Molina, I., y Clemente-Estevan, R.A. (EN PRENSA/IN PRESS). Autism and Faux Pas. Influences of Presentation Modality and Working Memory. Spanish Journal of Psychology.

Estudio 4. Garcia-Molina, I., Clemente-Estevan, R.A. \& Andrés-Roqueta, C. (EN REVISIÓN/UNDER REVIEW). Moral judgements in autistic children. Moral Transgressions and Faux Pas stories. Research in Autism Spectrum Disorders. 
Capítulo 2 Marco teórico 


\subsection{Marco teórico}

Una de las preguntas que podrían resumir el objeto de estudio de esta tesis sería: ¿Pueden los niños y niñas con autismo desarrollar la capacidad de atribuir mente a los otros para realizar así juicios morales adecuados?

Para responder a esta pregunta, en el presente trabajo se tratará de descomponer la acción intencionada en los elementos que la constituyen con el propósito de explicar cómo nuestros estados mentales pueden causar eventos físicos, $\mathrm{y}$, a su vez, esto tiene un impacto en la mente de otras personas. De esta forma, se facilitará el reconocimiento del peso mental en el juicio moral derivado de diversas situaciones que a continuación se detallan.

Muchas han sido las disciplinas, además de la psicología, que han estudiado cómo los humanos razonan moralmente sobre sus acciones y sus posibles consecuencias, así como también generan juicios sobre las acciones de los otros. Para dicho estudio, se han barajado diversas combinaciones de factores que pueden afectar a la total comprensión de un comportamiento o un escenario formado por valencias positivas o negativas en intención y resultado, que pueden derivar en situaciones intencionadas, accidentales, fallidas o, simplemente, benignas. En el caso de los diferentes estudios que se presentan, éstos se centran en situaciones intencionadas y accidentales.

Como novedad, en la presente tesis se aporta la relación entre diversas situaciones intencionadas y accidentales que no se ha hallado en trabajos anteriores, como sería la evaluación y razonamiento moral de una situación accidental (las meteduras de pata). Dicha comprensión se basaría en atribuir capacidades mentalistas - especialmente intenciones - a los personajes de las diferentes situaciones, hecho que puede resultar bastante complejo para niños y niñas con autismo. Por ello, como colofón, se reconocen diversas variables que pueden influir y ayudar para una mejor comprensión de este tipo de situaciones diarias o tareas mentalistas, proporcionando así un plus en la contribución social que esta tesis pretende aportar a la comunidad autista.

\subsubsection{Teoría de la Mente y juicios morales}

Los seres humanos somos expertos en - como decía Baron-Cohen (1997) - 'leer la mente', atribuyendo mente a las demás personas y actuando en consecuencia, a sabiendas de que el otro también puede entender la nuestra. Las personas tenemos más habilidades para 
discernir qué es lo que los otros perciben, desean, intentan, saben o creen, que cualquier otra especie, y es justamente ésta una de las habilidades más relevantes de entre aquellas que nos distingue de los animales (Ver Goldman, 2012). Si dicha capacidad - la de teorizar sobre la mente de los demás - se representara de forma piramidal, en el pináculo encontraríamos las creencias, y como habilidad fundamental, en la 'base' de la pirámide, las intenciones (Tomasello, Carpenter, Call, Behne y Moll, 2005); pues si bien entender y razonar acerca de las intenciones ajenas nos permite interpretar las acciones de los demás (Grant y Mills, 2011; Juhos, Quelhas y Byrne, 2015; Tomasello et al., 2005; Walsh y Byrne, 2007), inferir y razonar acerca de las creencias de los otros nos posibilitaría la comprensión del mundo interno-mental y su distinción del mundo físico-real (Wellman, Cross y Watson, 2001, para revisión).

Esta habilidad mentalista que nos permite detectar las intenciones, emociones, deseos y creencias ajenas es la llamada Teoría de la Mente (TM, a partir de aquí), y es muy necesaria en nuestro día a día, haciéndonos capaces de entablar relaciones sociales, evaluar los comportamientos de los otros y juzgarles, o incluso decidir qué personas nos convienen y cuáles no (Young y Tsoi, 2013). Así pues, este razonamiento mentalista, nos permitiría tomar consciencia de nuestras decisiones morales, confirmando, tal y como algunos estudios han remarcado, la asociación entre TM y juicios morales (Malle, 2004; Knobe, 2005; Pettit y Knobe, 2009; Young, Cushman, Hauser y Saxe, 2007; 2010; Young y Saxe, 2009).

Los estudios evolutivos señalan que la capacidad para entender uno de los precursores más complejos en la comprensión de la mente es la creencia ajena, y suele iniciar su desarrollo alrededor de los 4 años (Callaghan et al., 2006; Gopnik y Astington, 1988; Liu, Wellman, Tardif y Sabbagh, 2008; Perner, Leekam y Wimmer, 1987; Wellman, Cross y Watson, 2001). Sin embargo, existe cierta controversia acerca de cuándo los niños alcanzan a comprender las intenciones. Algunos autores remarcan fechas próximas al primer cumpleaños (Tomasello et al., 2005; Cushman, Sheketoff, Wharton y Carey, 2013), incidiendo en que, durante la infancia, los bebés empiezan a demostrar que pueden entender una acción como intencional, percibiendo y entendiendo que las personas: (1) generan su propio movimiento - y los objetos, no; (2) tienen un objetivo y persisten hasta realizarlo; y (3) consideran planes y eligen el más oportuno intencionadamente para realizar la acción (Tomasello et al., 2005). Sería este tipo de intención la que se nombraría anteriormente en la base de la pirámide, sin embargo, existe cierta controversia desde los inicios de las investigaciones sobre TM.

Muchos autores han investigado esta cuestión, siendo dos las aportaciones más distinguidas en relación con la intención. Una de las líneas de investigación se ha focalizado en acciones de carácter social e interactivo, en relación con el uso de gestos comunicativos o 
la capacidad de seguir a las personas con la mirada, practicar formatos interactivos, o comprender palabras. Esto pues, indicaría que las otras personas son agentes intencionales (Bretherton, 1991; Tomasello 1995). Otro enfoque teórico consideraría que los bebés tienen sistemas innatos para detectar la acción intencional recogiendo la capacidad temprana de dirigirse hacia metas, contacto perceptivo o reciprocidad. Entre los autores de esta segunda corriente se encuentran Baron-Cohen o Premack (Baron Cohen, 1995; Premack 1990). Al margen de explicaciones causales, parece claro que los bebés, antes de introducirse en las complejidades de comprender las dificultades de la relación comunicativa interpersonal, manifiestan tener intención.

Respecto a la comprensión de la intención para establecer juicios morales, ésta se manifiesta más tardíamente, iniciándose con la aportación adulta en la crianza. Así, se reconoce que las familias (y otros adultos) hacen más comentarios verbales sobre las transgresiones morales a los niños de 3 años que a los de 2 años (Smetana, 1989) desaprobando y justificando las diferencias entre acciones intencionales o accidentales a pesar de la incomprensión infantil.

Para la presente tesis, se partirá de la idea de que la TM nos permite comprender las intenciones de los demás, entendiendo la intención como el interés por conseguir unos deseos y la planificación de su logro junto a la creencia de las posibilidades para realizar una acción. En base a entender dichos estados mentales (y con ellos, la conformación de la intención) podremos realizar un juicio moral adecuado, siguiendo así una línea más acorde con los estudios clásicos, como el trabajo seminal de Piaget, en 1932.

Piaget, inauguró con dicho estudio ('The moral judgment of the child') la psicología moral, reportando un cambio en el desarrollo del niño entre los 6 y los 10 años, edad en la cual los niños empezarían a basar sus juicios morales en la intención, en vez de, en el resultado final. Para ello, presentó una serie de historias en las que un personaje, accidentalmente (intención), realizaba daños materiales graves - verter una gran cantidad de tinta en el escritorio - (resultado final); y otro que, de manera intencionada y maliciosa (intención), causaba daños menos graves - verter unas gotas de tinta en el escritorio - (resultado final). A continuación, se les preguntó a los niños qué personaje había sido más malo y si debería ser castigado. Esta pregunta acerca de la maldad o moralidad del agente permitió entrever el cambio evolutivo en el niño que iría de elaborar un juicio moral basando su razonamiento en el resultado a basarlo en la intención. Mientras los niños más pequeños considerarían moralmente peor un daño grave pero accidental (basándose en el resultado), los niños más mayores considerarían peor un daño menos grave pero intencionado (basándose en la intención). Dicha evolución comprensiva en 
la que se tienen en cuenta los estados mentales del agente (sus intenciones, deseos y creencias) en vez de los actos más tangibles (como el resultado final) ha sido ampliamente estudiada en trabajos posteriores a Piaget, con hallazgos similares (Armsby, 1971; Baird y Astington, 2004; Costanzo, Coie, Grumet, y Farnill, 1973; Helwig, Hildebrandt, y Turiel, 1995; Imamoglu, 1975; Killen et al., 2011; Moran y O’Brien, 1983; Nobes, Panagiotaki, y Pawson, 2009; Surber, 1977; Wainryb, Brehl, y Matwin, 2005; Yuill, 1984; Yuill y Perner, 1988; Zelazo, Helwig, y Lau, 1996).

Si bien se han encontrado resultados de manejo de la intención más precoces (Chandler, Sokol y Hallet, 2001) y se ha criticado la metodología utilizada en el trabajo piagetiano, en líneas generales, los niños pequeños suelen basar su juicio en relación al resultado final, corroborando así que la evaluación moral tiene, como pensaba Piaget, una evolución marcada por la edad.

Parte de la investigación reciente ha centrado su enfoque en comprender los estados mentales en el sentido de afirmar que tener TM es un requisito previo para el juicio moral, puesto que los niños y niñas necesitan poder reconocer las intenciones de un agente para realizar las evaluaciones morales correspondientes (Smetana, Jambon, Conry-Murray, SturgeApple, 2012).

Esta dificultad que experimentan niños y niñas en épocas tempranas para comprender transgresiones morales de valencias distintas, respondería a una falta de coordinación entre la comprensión de los estados mentales de los otros y el raciocinio moral propio derivado, puesto que ambas se presentan a la vez en una transgresión. Como ejemplifica Smetana, (2006) los niños pequeños rechazan la conducta de "pegar" al margen de la intencionalidad del perpetrador, puesto que razonan sobre el daño a la víctima y no sobre el estado mental del agente activo ('el que pega').

Por consiguiente, dada la importancia de la TM en los juicios morales, cabría remarcar si, así como los niños más pequeños, aún inmaduros en TM, tienen problemas en evaluar moralmente al agente 'perpetrador' o los diferentes elementos de la situación, también los individuos con un diagnóstico de Trastorno del Espectro Autista (TEA) podrían tener problemas en basar sus juicios morales en intenciones, dado su déficit en TM (Abell, Happé y Frith, 2000; Baron-Cohen et al., 1985, 2000; Bowler, 1992; Castelli, Frith, Happé y Frith, 2002; Margoni y Surian, 2016; Surian y Leslie, 1999).

Sin embargo, aunque los primeros indicios apuntarían a un déficit en TM que repercutiría en el juicio moral, tradicionalmente, los estudios iniciales acerca de juicios morales 
y autismo, se centraron en la distinción entre transgresiones morales y convencionales, o contemplaron el razonamiento acerca de una acción que estuviese bien o mal (ver crítica en Killen et al. 2011; o Margoni y Surian, 2016). Por esta razón, sería necesario analizar detalladamente estos aspectos morales en los que se focalizaron los primeros estudios 'menos mentalistas' y que evidenciaron un aspecto fundamental en la formación de la competencia moral.

\subsubsection{Transgresiones morales y convencionales}

¿Qué está bien y qué está mal? Para poder responder a esta pregunta aparentemente sencilla se deberían tomar en consideración diversos aspectos.

En término generales, se debería identificar si se trata de una transgresión moral universal, es decir, si el código por el que las personas se rigen para evaluar un acto como correcto o incorrecto moralmente es independiente de su nacionalidad, religión, edad, o género (ver DeScioli, Asao y Kurzban, 2012; Haidt, 2007; Hauser, 2006; Mikhail, 2007; Stich, Fessler y Kelly, 2009). Esto, aunque parezca extraño, no significa que todos los seres humanos de este planeta acepten y sigan las mismas normas morales, sino que existe una predisposición a evaluar moralmente de una forma similar - según Kahane (2010), esto sería comparable con la idea de Chomsky de que todos los lenguajes subyacen a una misma estructura gramatical.

En esta misma línea, otro aspecto clave en la moralidad y que ha sido ampliamente estudiado en niños pequeños o con algún tipo de condición como el autismo, es la distinción entre transgresión moral y transgresión convencional. Se podría pensar en un ejemplo de transgresión convencional, como el de un niño bebiendo su sopa directamente del bol, sin usar cuchara. A diferencia de la transgresión moral (como matar a otra persona), la acción descrita, considerada convencional, no contemplaría una 'víctima'; y, por ende, la transgresión moral estaría considerada más grave que la convencional. También, la permisibilidad y la autoridad tendrían un peso importante, pues las transgresiones morales son 'menos permisibles' que las convencionales y son independientes de la autoridad (el niño puede tener permitido beber directamente del bol). Además, aunque con cierta polémica cultural implícita, las transgresiones morales se considerarían de alcance general, es decir, su maldad o incorrección sería extensible a diferentes lugares y tiempos; mientras que las convencionales serían más locales, y su maldad o incorrección sería subyacente al lugar o momento en el que ocurren (Sousa, 2009). 
La presente tesis, aunque en una dimensión más cotidiana para los niños se basa en transgresiones morales (romper, robar, pegar, mentir...), las cuales implican que la víctima es lastimada física o psicológicamente y sometida a una injusticia y/o violación de los derechos básicos e importantes para el ser humano (Sousa, 2009).

Evolutivamente, es sobre los 3 - 4 años que los niños con desarrollo neurotípico (NT) empezarían a juzgar las transgresiones morales más duramente y menos dependientes de la figura autoritaria que las transgresiones convencionales (Nucci, 1987; Smetana 1981, 1985; Smetana y Braeges, 1990; Turiel, 1978). Aun así, existe cierta controversia acerca de si los niños preescolares son capaces de distinguir dicho binomio debido a la presencia de daño a la víctima en unas - morales - y ausencia en otras - convencionales - (Smetana, 1983; Turiel, 1983).

Al igual que los niños pequeños - con una TM inmadura - en lo relativo a los individuos con autismo, se aventuró, en estudios sobre moral ampliamente citados, que éstos podían realizar la distinción entre transgresiones morales y convencionales (Blair, 1996; Leslie, Mallon y DiCorcia, 2006). Sin embargo, literatura más reciente sugeriría la necesidad de una comprensión mentalista más madura para deducir qué intenciones pueden llevar a una persona a causar una acción y de este modo juzgar correctamente una transgresión (ya sea moral o convencional) (Buon, Dupoux, Jacob, Chaste, Leboyer y Zalla, 2013; Margoni y Surian, 2016; Moran et al., 2011).

Así pues, recientemente se cuestionó si los individuos con autismo eran realmente capaces de distinguir entre transgresiones morales-convencionales o existía un sesgo en sus juicios debido a factores externos irrelevantes o de los que no dependería la real comprensión de una transgresión. Por ejemplo, por la afectación del nivel emocional de los agentes implicados (la víctima llora), y no a factores más internos o mentales como la acción intencionada. En esta línea, el estudio de Weisberg y Leslie (2012) halló que, contrariamente a los niños NT, los niños con autismo parecerían juzgar más severamente las acciones en las que la víctima llora, que las mismas acciones que no causan el lloro de nadie. Por consiguiente, aunque los primeros estudios nos remarcarían el hecho de que las personas con autismo podrían hacer la distinción moral-convencional, cabría preguntarse cómo hacen esta distinción y si dicha conclusión no fue 'simplista' sin tener en cuenta otros factores que podrían estar afectando su juicio moral.

Una de las hipótesis que respaldaría dicha afirmación - y los estudios que se presentan en esta tesis - sería la que presentaron en su trabajo Margoni y Surian, (2016), los cuales 
destacaron que los niños con autismo responderían de forma similar a los niños NT cuando los casos fueran simples e inequívocos (entiéndase inequívoco como situaciones con valencias no ambiguas: resultado negativo producido por una intención negativa). Así pues, siguiendo esta hipótesis, los niños con autismo parecerían tener desarrollada la comprensión de juicios morales básicos - no ambiguos - aunque, posiblemente esto estaría enmascarado por la poca presencia de aspectos mentalistas en este tipo de tareas o cuestiones relacionadas (por la coordinación directa entre los estados mentales en estas tareas y las cuestiones relacionadas), por su atención preferente a variables externas no concluyentes (como el lloro), o por la noambigüedad en las valencias de resultados e intención.

\subsubsection{El peso de los estados mentales en los razonamientos morales}

\subsubsection{De lo mental al resultado y su posterior análisis moral}

La teoría causal de la acción intencionada sería uno de los hallazgos más antiguos y mejor documentados en el campo de la psicología moral, que como muchas investigaciones han remarcado tiene un papel fundamental en los procesos involucrados en los juicios morales, y que contempla deseos, creencias e intenciones en su desarrollo (Alicke, 2000; Cushman, 2015; Heider, 1958; Kiley-Hamlin, Ullman, Tenenbaum, Goodman y Baker, 2013; Malle, Guglielmo, Monroe, 2014; Mikhail, 2011; Weiner, 1995; Young y Tsoi, 2013).

Constantemente, atribuimos ‘intención’ al comportamiento ajeno (Malle, 1999; Baker, Saxe y Tenenbaum, 2009), ya que tendemos a explicar eventos físicos, visibles u observables como consecuencia de unos estados mentales; es decir: primero pensamos nuestro objetivo deseado y maquinamos un plan con intención, y luego producimos la acción para conseguir dicho objetivo como resultado. Así pues, tal y como ya se había citado en la explicación de la TM, si somos capaces de atribuir deseos, creencias e intenciones a los demás (y a nosotros mismos) podemos predecir, intuir y comprender conductas propias y ajenas; y, lo que más repercute en la moralidad, razonar acerca de los elementos implicados: agente, acción, resultado.

En sí, debemos reconocer que el resultado físico es producto de un estado / objetivo mental. Para entender cómo un deseo (objetivo mental) se convierte en un hecho físico, es necesario hablar de los elementos que conforman la intención, y para ello el concepto de 'acción intencionada' es particularmente relevante en cuanto a la formación de nuestros juicios morales (Cushman, 2015; Zalla y Leboyer, 2011). Para explicar dicho modelo, es necesario 
entender los elementos que conforman una acción con intención deliberada, centrándonos ante todo en los aspectos más mentales del agente. Según Malle y Knobe (1997), se deben considerar cinco principios en los que 'el agente debe': (1) desear el resultado de la acción; (2) creer que su acción llevará a ese resultado; (3) tener la intención de realizar la acción; (4) ser consciente de su acto mientras ejecuta la acción; y (5) tener las habilidades necesarias para realizar dicha acción.

Como se observa, el agente es el elemento principal por el que ocurre la acción: sus deseos, creencias, pensamientos, mecanismos e intenciones son aspectos iniciales en el proceso de una acción intencionada (aspectos no observables). Consecuentemente, todos estos estados mentales formarán una acción que tendrá el resultado deseado (aspecto observable).

Pero, además, se podría añadir un sexto elemento, siguiendo el paradigma de Tomasello et al. (2005), que sería (6) la consideración de nuestra realidad actual en el modelo 'mental' (aspecto no observable). Con el propósito de entender dicho paradigma mejor, en el estudio de Tomasello et al. (2005) se describió el ejemplo de cómo abrir una caja de cartón, siendo el objetivo deseado abrir esa caja de cartón, y la realidad de la situación del agente que dicha caja estaba cerrada y de qué forma creía el agente que estaba cerrada. Las opciones acerca de la realidad de esta caja cerrada podrían añadirse por el contexto, por ejemplo, la caja está cerrada con cinta adhesiva, y esta sería pues, nuestra creencia sobe la realidad. Siendo conscientes de esta creencia de la realidad y teniendo en cuenta conocimientos y habilidades, se podrá planear cómo abrir la caja y, de ese modo, conseguir el resultado deseado.

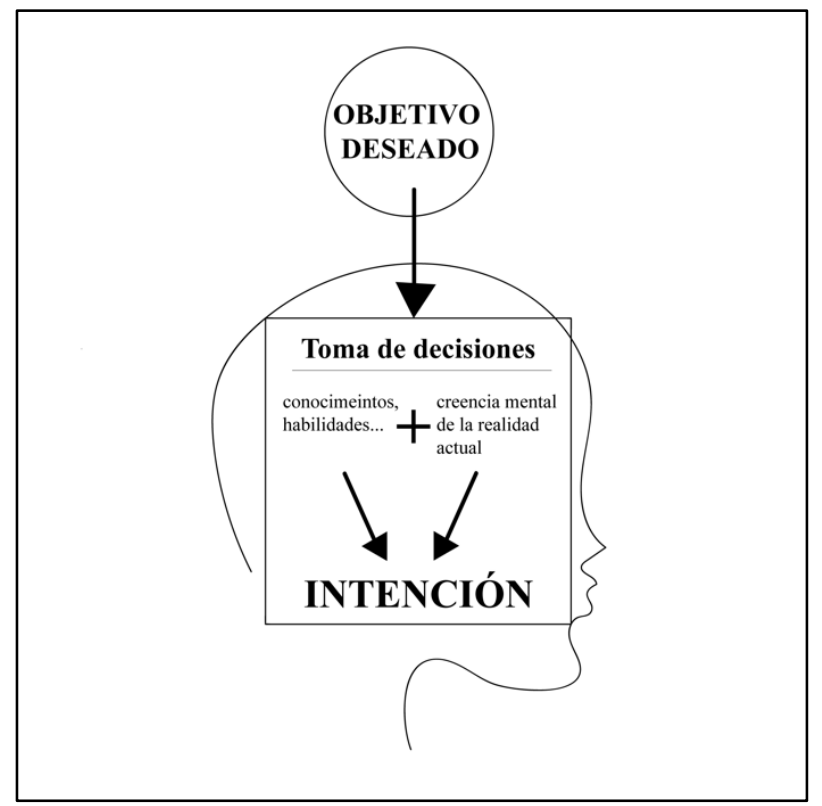

Figura 2-1. Modelo sobre cómo se forma la intención, extraído de Tomasello et al. (2005). 
Esta toma de decisiones (Figura 2-1) es conocida también como plan. Un plan es la representación mental de una acción (o de varias acciones) que deben llevarse a cabo para conseguir un resultado deseado (Cushman, 2015). Planificar implica una serie de decisiones intencionadas que pueden hacer que dicho plan sea muy fácil o muy difícil. Un ejemplo de plan ‘complicado' sería preparar una mudanza, mentir, robar una manzana de una tienda o cometer un homicidio. Estos planes estarían construidos para dar respuesta a unos deseos (se tiene el deseo de tener más espacio y una casa mejor) y unas creencias basadas en el resultado deseado de dicha acción (si se realiza la mudanza a esta otra casa se podrá tener más espacio y una casa mejor). Por eso, cuando se dice que una persona actúa intencionadamente, deliberadamente, voluntariamente o a propósito, se puede entender que está actuando según su plan (Cushman, 2015).

El análisis del modelo de acción intencionada tiene un alto impacto en el razonamiento moral de una situación: saber si una persona es buena o mala, si una acción es correcta o incorrecta, o qué tipo de castigo merece una persona, está asociado a la atribución de estados mentales, y, por tanto, a la intención de la persona que causa la acción. Para entender mejor dicha asociación intención - moralidad, el trabajo de Cushman (2015) nos ofrece de forma detallada la descomposición del modelo de acción intencionada y la reconstrucción y relación con la moralidad, a través de un ejemplo específico (Figura 2-2. Descomposición de una acción intencional basada en la transgresión del veneno (Cushman, 2015).

El delito que se explica es el de que una persona sabe que va a heredar de su tío una buena suma de dinero una vez éste muera (creencia). Esa persona desea el dinero y realmente no le importa su tío (deseo). Entonces, planea envenenarle deliberadamente para poder conseguir su dinero (plan - intención). Conscientemente y con el veneno necesario (control) pone el veneno en el café del tío (acción). Dicha acción tiene como consecuencia la muerte de su tío; y, por tanto, el dinero es heredado (resultado). 


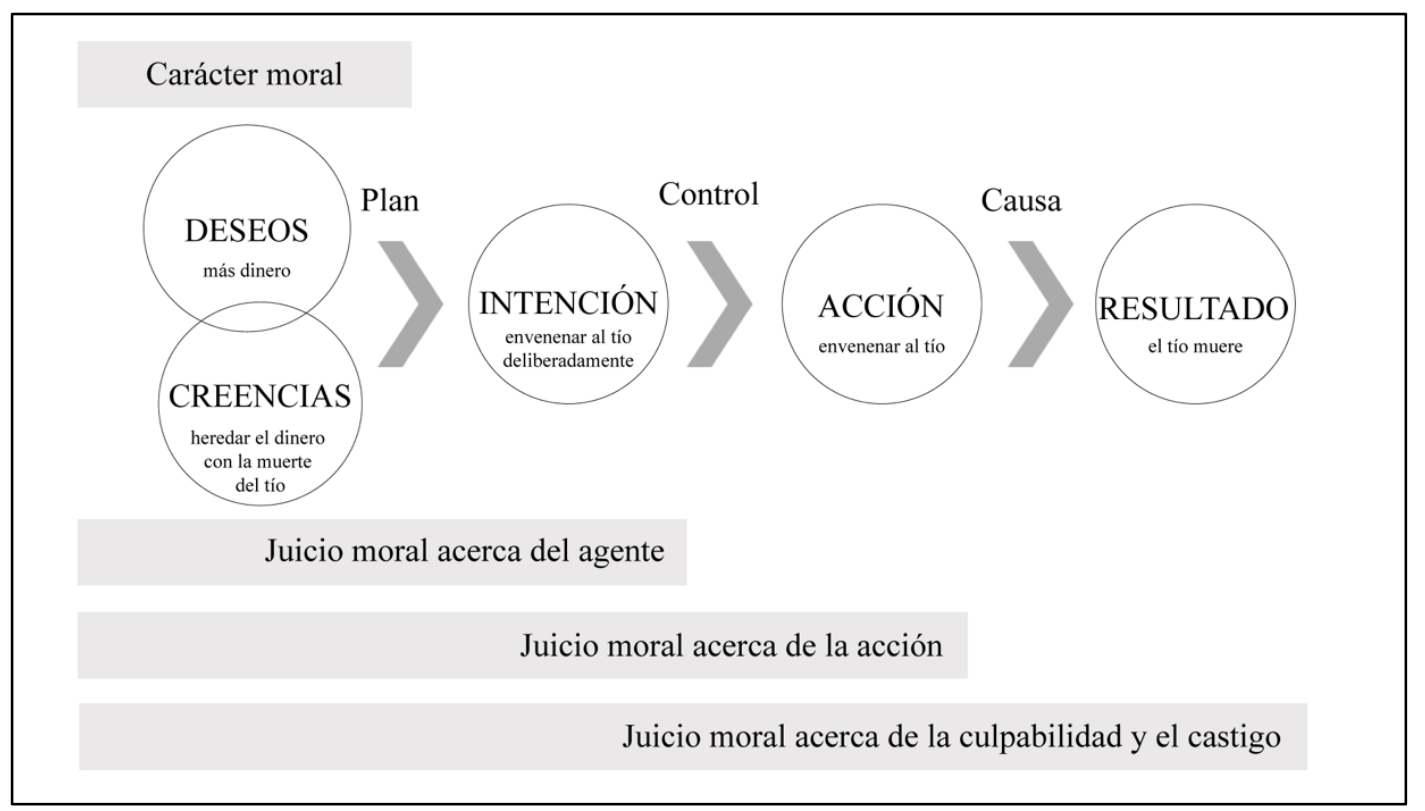

Figura 2-2. Descomposición de una acción intencional basada en la transgresión del veneno (Cushman, 2015).

Este ejemplo de valencias no-ambiguas sería considerado una transgresión moral básica. De hecho, desde el razonamiento moral, envenenar a alguien sería un delito grave, ya que violaría un derecho universal. Sin embargo, la comprensión total de la transgresión recaería en entender los estados mentales del agente activo que planifica el envenenamiento, hecho por el cual sería necesaria una TM madura. De manera importante, atribuir posibles estados mentales a los otros, más concretamente, la inferencia de deseos (querer matar a alguien porque se desea tener más dinero), permitiría juzgar la 'moral' del agente perpetrador de la acción. Más concretamente, juzgar el carácter moral de esa persona (Chakroff y Young, 2015; Inbar, Pizarro y Cushman, 2012). Entiéndase 'carácter moral' como un conjunto de virtudes culturalmente definidas, como no romper una norma (Hartshorne y May, 1930).

En resumen, y siguiendo la Figura 2-2, se destacaría que, el juicio acerca del carácter moral de una persona dependería de los deseos de esta persona. Los juicios acerca de la 'maldad' o ‘incorrección' del acto (wrongness en inglés) dependerían de la acción realizada, así como de los deseos y creencias de la persona que realiza la acción; y los juicios sobre el castigo merecido dependerían de la acción y los estados mentales de la persona, además del resultado o consecuencia de dicha acción (Cushman, 2015). Aunque la mayor parte de los estudios se centran de forma general en analizar la situación o la transgresión presentadas, recientemente en el ámbito de la moralidad, el foco de estudio se encuentra en la intención para 
culpar o juzgar al personaje que realiza la acción. Por eso, se ha añadido a la base original de procedencia de la Figura 2-2 el 'juicio moral acerca del agente', el cual dependería de sus intenciones reales, es decir, de la planificación de sus estados mentales (deseos y creencias).

El análisis descrito en la Figura 2-2 sería también necesario para explicar situaciones en las que resultado e intención no tienen la misma valencia, como en el caso de accidentes o intentos fallidos, en los que existiría un mayor nivel de dificultad en términos mentalistas por ser sus valencias ambiguas.

\subsubsection{Las valencias ambiguas y su repercusión en el juicio moral}

Cuando intención y resultado tienen valencias diferentes en una misma situación, se añade un plus de dificultad al razonamiento moral, ya que la intención del agente tiene un peso muy importante, requiriéndose así un análisis más exhaustivo de los estados mentales, con un menor peso de las consecuencias externas y observables de la acción (Margoni y Surian, 2016). Este sería el caso de los intentos fallidos y las situaciones accidentales. En el primer ejemplo, el caso de los intentos fallidos, un ejemplo concreto podría ayudarnos a discernir la importancia de los estados mentales y su repercusión en nuestro juicio moral sobre el agente. El ejemplo sería el siguiente: un niño tiene el 'deseo' durante años de matar el pájaro de su hermana, y un día lo intenta deliberadamente, pero en ese momento escucha que viene su madre y el niño huye, así que, finalmente, el pájaro no muere. Aunque el niño no haya conseguido su objetivo deseado, la mera presencia del deseo malicioso describirá más acerca del carácter moral del niño que el resultado final (Cushman, 2015; Young y Tsoi, 2013). Así pues, aunque el 'asesinato' del pájaro fue fallido, si somos capaces de reconocer su intención 'maliciosa' podemos reconocer la maldad del niño, pues, la información acerca de las intenciones, creencias y deseos, a menudo, dominan nuestros juicios morales (Young y Tsoi, 2013).

Sin embargo, aún cabría remarcar otra dimensión a tener en cuenta en este tipo de historias de valencias ambiguas, y sería la culpabilidad. En el caso del ejemplo anterior, no existe resultado bueno o malo, simplemente, la acción es fallida (o no controlada) y, como consecuencia, no ocurre el resultado deseado. Pero, ¿qué pasaría si ocurre algo inesperado, como un resultado malo, cuando no había intención deliberada?

Rectificando parte del ejemplo anterior del veneno, ¿qué ocurriría si la persona que hace el café hubiese echado el veneno en la taza pensando que ese polvo blanco era azúcar? 
En ese caso, la persona que hace el café no estaría controlando la acción (envenenar a su tío), tampoco tendría la intención deliberada de matarle, ya que su creencia sobre la realidad sería falsa (ella cree que el polvo es azúcar) y su deseo tampoco sería matarle. Como se detalla, una situación accidental es definida por más elementos que las situaciones fallidas, aunque, tal y como se puede ver en la Figura 2-3, ambas situaciones parten de tres factores clave, dos de ellos tomados de estudios previos: intención y resultado (Cushman, 2015; Martin y Cushman, 2016; y Young et al., 2007), y como novedad, en esta tesis se ha añadido al constructo un tercer factor: el control de la acción. Dicha combinación de factores, no es la que comúnmente encontramos en los estudios relacionados con el tema, puesto que la acción suele quedar implícita en la intención. Aún así, dada la oportunidad de este tercer factor añadido (control de la acción), podemos observar más concretamente que cuando las valencias en intención y resultado concuerdan, existe un control que repercutirá en que, esos deseos generen el resultado físico esperado a través de un control de cada acto. Por el contrario, cuando no existe un control de la acción, el resultado no será el esperado debido a diversas causas, que irán desde lo mental (una falsa creencia - no-control de la realidad) hasta lo físico (no-control del acto). Por ejemplo, no será lo mismo no disponer de toda la información para saber que acabas de echar veneno en la taza de café, que saber que estas echando veneno en la taza, pero en el último momento alguien tira el café al suelo y nadie llega a bebérselo. El control o no-control de la acción se puede tildar pues de suerte, aleatorización, efecto o, incluso responsabilidad.

Ciertamente, los deseos y las intenciones son importantes para evaluar una transgresión, pero, tal y como se vio en el primer modelo (Figura 2-1) según Tomasello et al. (2005), la información acerca de la realidad actual sería un factor clave a contemplar, ya que dicha creencia, tal y como creyó el personaje principal, podría ser falsa (creencia falsa: cree que el polvo blanco es azúcar, cuando realmente es veneno) y a partir de dicha creencia acerca de la realidad, sería que se desencadenaría toda la serie de catastróficas desdichas conocida en el ejemplo del veneno-azúcar. Es decir, la distinción de la intención (como deliberada o accidental) tendrá una gran importancia en el razonamiento moral, ya que un daño que ha sido planeado deliberadamente será considerado 'más malo’ que un daño accidental. 


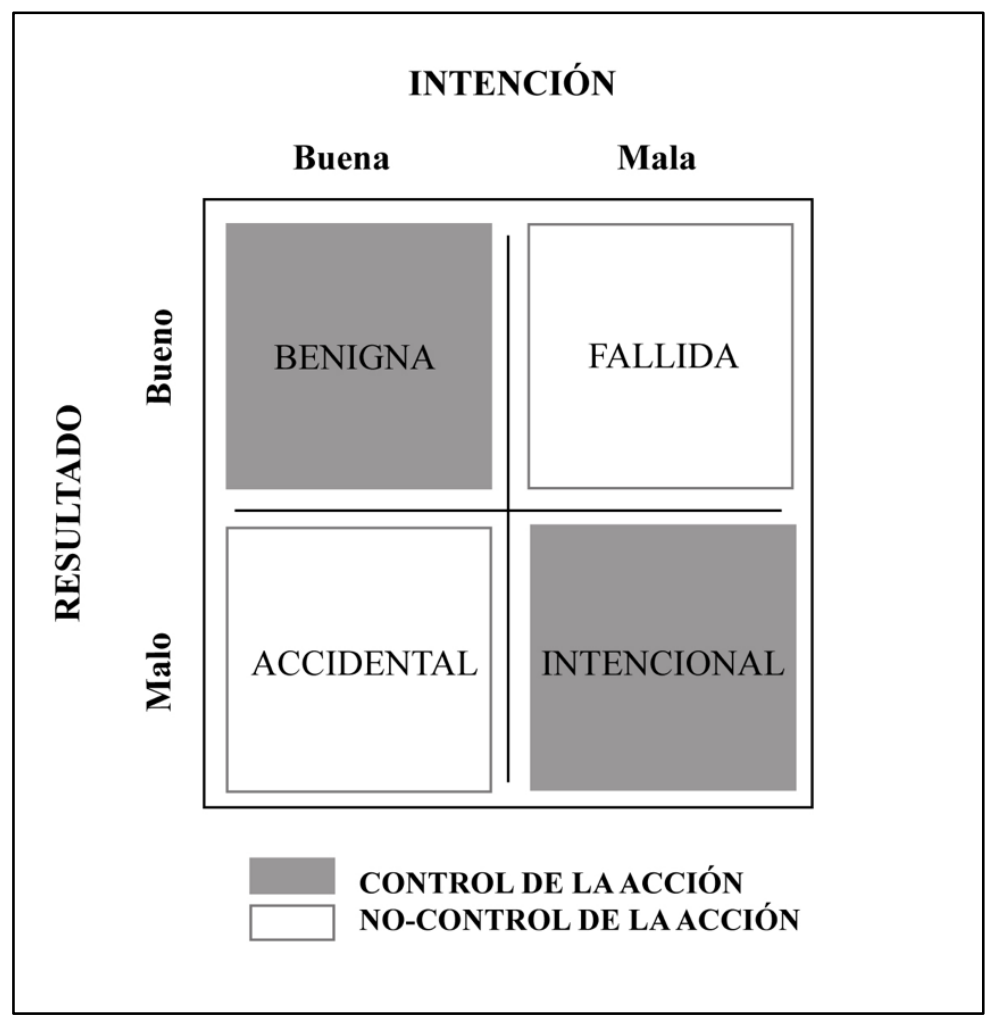

Figura 2-3. Situaciones que surgen de la combinación de los factores: intención, resultado y acción. Adaptado de Cushman (2015); Martin y Cushman (2016); y Young, Cushman, Hauser y Saxe (2007).

En líneas generales, los tratados de derecho contemplan estas diferencias. Así, si alguien mata a una persona deliberadamente (asesinato) será juzgado peor ('más malo, más culpable') que alguien que mata a una persona accidentalmente (homicidio involuntario) (Lagnado y Channon, 2008). Esta diferencia radica en la intención del perpetrador que lleva a cabo la acción (y en sus intenciones, creencias y deseos), aunque ambas situaciones tengan el mismo resultado sobre la víctima (la víctima muere en ambos casos).

En estas situaciones más complejas de valencias ambiguas, se encontró en varios estudios que los niños NT confiaron principalmente en el resultado para basar su juicio (Helwig et al., 1995; Helwig, Zelazo y Wilson, 2001; Zelazo et al., 1996) o en ambos, intención y resultado (Cushman et al., 2013; Killen et al., 2011; Nobes, Panagiotaki, y Bartholomew, 2016). Dos de los estudios más citados en esta área, son los de Helwig et al. (2001) y Zelazo et al. (1996), en los que se presentaron una serie de historias cuyas situaciones variaban en sus valencias - positivas o negativas - en intenciones y resultados. Después de cada historia, se preguntó a los niños acerca de la 'aceptabilidad de la acción' - tildado como incorrección o 
juicio de la acción en la presente tesis - (por ejemplo: ‘Está bien que Teresa le dé a Helena una tarántula?') y sobre el 'castigo' (por ejemplo: ‘¿Teresa se habrá metido en problemas?’). En sus resultados se destacó que el $68.7 \%$ de los niños entre 3 y 7 años (estudio de Helwig) y el $80.7 \%$ de los niños entre 3 y 5 años (estudio de Zelazo) basaron el juicio del acto en los resultados. Ningún niño de los dos estudios basó el juicio de la acción solamente en la intención. Por lo que se refiere al castigo, ambos estudios reportaron que los niños juzgaron los daños accidentales (el vendedor se equivocó y puso en la bolsa del regalo una tarántula) como 'más castigables' que los daños fallidos con intención deliberada (Teresa le quería asustar con una tarántula, pero a Helena le encantan las tarántulas), hecho que corroboraría otra vez la gran influencia del resultado por encima de la intención cuando la TM aún es inmadura.

\subsubsection{El peso de los estados mentales en los razonamientos morales de las personas con autismo}

Así como ocurre con los niños pequeños, por lo que se refiere a las personas con autismo, varios hallazgos parecen clarificar que suelen juzgar las transgresiones en base a los resultados (o consecuencias), en vez de según las intenciones del agente, debido a sus dificultades en TM (Margoni y Surian, 2016; Young y Tsoi, 2013; Zalla y Leboyer, 2011. Consultar Figura 2-4 extraída de Margoni y Surian, 2016). Sin embargo, por otro lado, hay estudios que destacan que los individuos con autismo son capaces de culpar más duramente a un agente que causa un daño intencionado de otro que lo hace accidentalmente, es decir, utilizan la información relativa a la intención para hacer un juicio moral sobre el agente (ver Grant, Boucher, Riggs y Grayson, 2005, o Rogé y Mullet, 2011).

En el caso del estudio de Grant y colaboradores, los niños con autismo juzgaron a una persona que hirió a otra persona como más culpable, que una que dañó un objeto perteneciente a alguien. Aun así, cabe remarcar que, en estos dos últimos estudios citados, las personas con autismo no resolvieron las pruebas tan bien como su grupo comparación, y, como en el caso del trabajo de Grant y su equipo (2005), sus explicaciones acerca de la moralidad fueron pobres y reiterativas (nombrando partes exactas de la historia escuchada). En este caso, menos de un tercio de los participantes hicieron referencia a la intención.

En el estudio de Rogé y Mullet (2011), en el cual se observaron varios grupos de edades (niños, adolescentes y adultos), se concluyó que la intención era más considerada en el grupo de adultos con autismo que en el de los niños - los cuales (niños con autismo y NT) tendieron a juzgar influenciados por el resultado (Cushman et al., 2013) 
Ciertamente, aunque el grupo TEA suele responder similar al grupo NT cuando se establecen variables de control intergrupal como inteligencia promedio o la correcta resolución de la creencia falsa - entre otras, las evidencias muestran que suelen existir diferencias entre grupos, sobre todo cuando se contemplan transgresiones de tipo accidental.

Por ejemplo, en Buon et al. (2013) y en Moran et al. (2011), los participantes con autismo difirieron de su grupo de comparación (NT) en los juicios sobre intención, llegando a juzgar más duramente al agente que hirió accidentalmente a la víctima que los adultos NT. Así pues, los participantes con autismo culparon más severamente al agente del daño accidental que su grupo de comparación. Este patrón de 'sobre-culpa' o de tendencia a considerar las intenciones más deliberadas de lo que realmente son, parece repetirse en situaciones accidentales cotidianas, como se verá más adelante con las meteduras de pata.

Aunque, como se ha visto anteriormente, existe cierta controversia referente a si el juicio moral de las personas con autismo se centra más en el resultado que en la intención, un estudio reciente de Koster-Hale, Saxe, Dungan y Young (2013) evidenció la activación de la unión tempo-parietal derecha en los sujetos con NT (el área asociada al razonamiento de los estados mentales - intenciones, pensamientos y creencias) durante la distinción de transgresiones accidentales versus intencionadas, activaciones que no se encontraron en los adultos con autismo. Los autores destacaron que no existieron diferencias neuronales en el grupo TEA cuando el daño era accidental o deliberado. Es decir, para los individuos con autismo, no existieron diferencias cuando los pares de situaciones de intención accidental o deliberada eran presentados. La activación neuronal era la misma independientemente de si el personaje le daba a un niño cacahuetes a sabiendas de que era alérgico y eso le provocaría la muerte (de forma deliberada), que cuando lo hacía sin saber que era alérgico y eso le provocaría la muerte (de forma accidental). Al parecer, dicho hallazgo seguiría en la línea de que las personas con autismo juzgarían la situación en términos de resultados finales, pues tendrían dificultades en discernir los estados mentales cuando se juzgan situaciones en las que las intenciones y los resultados presentan valencias diferentes (Margoni y Surian, 2016). En la Figura 2-4 encontramos un resumen de ello. En la clasificación que se detalla, se observa en qué se basarían las personas con autismo en comparación con las de desarrollo NT para juzgar una transgresión cuando la acción es deliberada o accidental, en referencia al mismo acto, así como también al agente. Las diferencias más abismales radicarían en el juicio de la culpabilidad del agente. En el caso de los individuos con autismo el juicio del agente estaría movido por el resultado final de la acción. En cambio, en las personas NT, el juicio estaría basado en la 
intención del agente. Así pues, la Figura 2-4 sería necesaria para entender repercusiones morales que se derivan de entender o no la intención del otro, como la culpabilidad o el castigo.

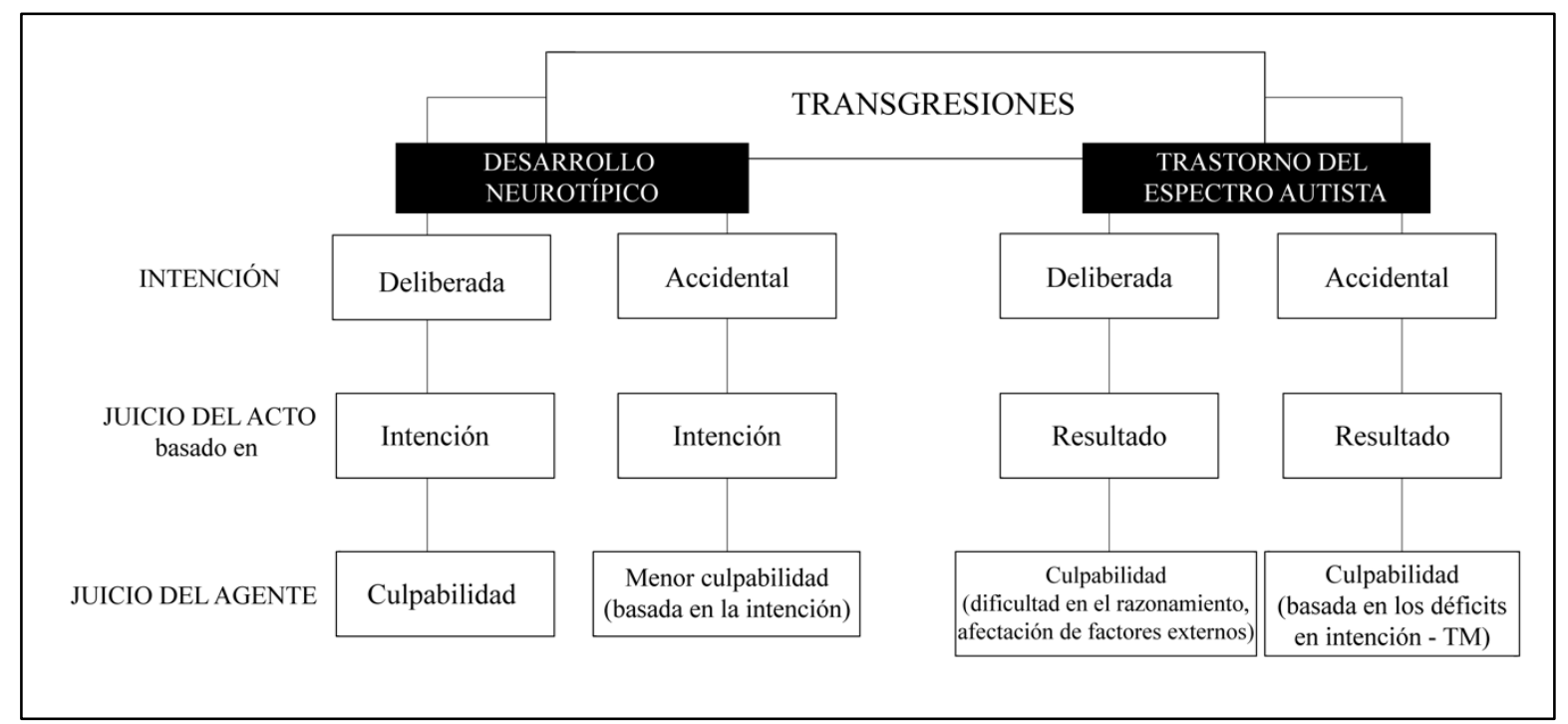

Figura 2-4. Comparación TEA - NT en los elementos que repercuten en una transgresión.

\subsubsection{Los juicios morales en las situaciones accidentales}

\subsubsection{Las meteduras de pata y los juicios morales}

A pesar de que la mayor parte de la investigación sobre este tema se ha realizado sobre dilemas morales, otras acciones de la vida diaria contemplan la capacidad humana de hacer daño de forma no intencional, en general por alguna característica mental del agente que impide o dificulta su controla de la intención (por la falta de atención, el desconocimiento de la información o la creencia errónea acerca de la situación en concreto). Este es el caso de las meteduras de pata, cuyos ejemplos son, sin duda, más habituales que un homicidio involuntario.

Aunque la metedura de pata ha sido contemplada en estudios para apreciar el peso de la intención, se remarca en Buon et al. (2013) la sugerencia de que una metedura de pata puede ser malinterpretada cuando el juicio acerca de la intención del agente está afectado por la valencia del resultado de la acción. Por dicha razón se ha considerado necesario incluir la metedura de pata en el presente trabajo para contemplar todas las ópticas que tiene el tema planteado. 
Una metedura de pata (traducción del término francés faux pas) se produce cuando existe un error social embarazoso o un desliz en la conducta, modales o reglas sociales (extraído de Garcia-Molina, et al., 2016). Según Baron-Cohen, O’Riordan, Jones, Stone y Plaisted, (1999), una metedura de pata podría resumirse por el 'Oh-oh' de reacción o el gesto de taparse la boca. Un ejemplo de metedura de pata, podría ser la siguiente historia (extraída de GarciaMolina et al., 2016, adaptada de Baron-Cohen et al., 1999, y utilizada en el estudio 2, 3 y 4. Para más ejemplos ver 7.1 Apéndice 1; 7.2 Apéndice 2 y 7.3 Apéndice 3.

Ismael le había comprado a Judit un avión de juguete para su cumpleaños. Meses más tarde, estaban jugando juntos con ese mismo avión, cuando accidentalmente, a Ismael se le cayó rompiéndose un ala. Entonces, Judit le dijo: 'No te preocupes, Ismael. De todos modos, nunca me gustó. Alguien me lo regaló por mi cumpleaños' (Garcia-Molina et al., 2016, p. 104).

Siguiendo el ejemplo, para entender bien dicha historia sería necesario, por un lado, detectar que el emisor (Judit) no recuerda o desconoce cierto hecho; y, por otro lado, apreciar el impacto emocional que puede producir en el oyente (Ismael) dicho comentario. Por tanto, existe una diferencia de nivel compartido de conocimiento entre emisor y oyente. Tal y como ya ocurría en el ejemplo del veneno - azúcar acerca de la creencia falsa de que el azúcar era realmente azúcar (y no veneno), en las meteduras de pata la creencia falsa de la realidad tendría un peso muy importante para su correcta comprensión. Así pues, Judit no sabe o no recuerda una información que Ismael sí sabe; por consiguiente, Judit tiene una creencia falsa de la realidad.

Si analizamos las meteduras de pata a partir del modelo de acción intencional que habíamos descrito anteriormente y esquematizado en la Figura 2-2, el hecho de que la creencia de la realidad sea falsa repercute en los otros elementos que se encadenan: la intención deja de ser deliberada, ya que el resultado de dicho comentario no fue premeditado o planeado y, el control de la acción no es tal, ya que el resultado es diferente al planeado en un inicio (cambia de informar al oyente a herir al oyente). Para una visión más general, ver Figura 2-5. 
Como ocurre en el ejemplo del veneno - azúcar, aunque el personaje principal tenga los mecanismos necesarios para poder realizar la acción, dicha acción no es controlada completamente, ya que existe una información oculta en la situación (Judit puede realizar el comentario, pero lo hace delante de la persona errónea y no controla totalmente la situación). Finalmente, este cúmulo de encadenamientos hace que el observador (quien tiene toda la información de tipo mentalista) llegue a la conclusión de que el resultado final ha cambiado totalmente como consecuencia de la creencia falsa del emisor (que a su vez repercute en la intención accidental), es decir, de que Judit, metió la pata.

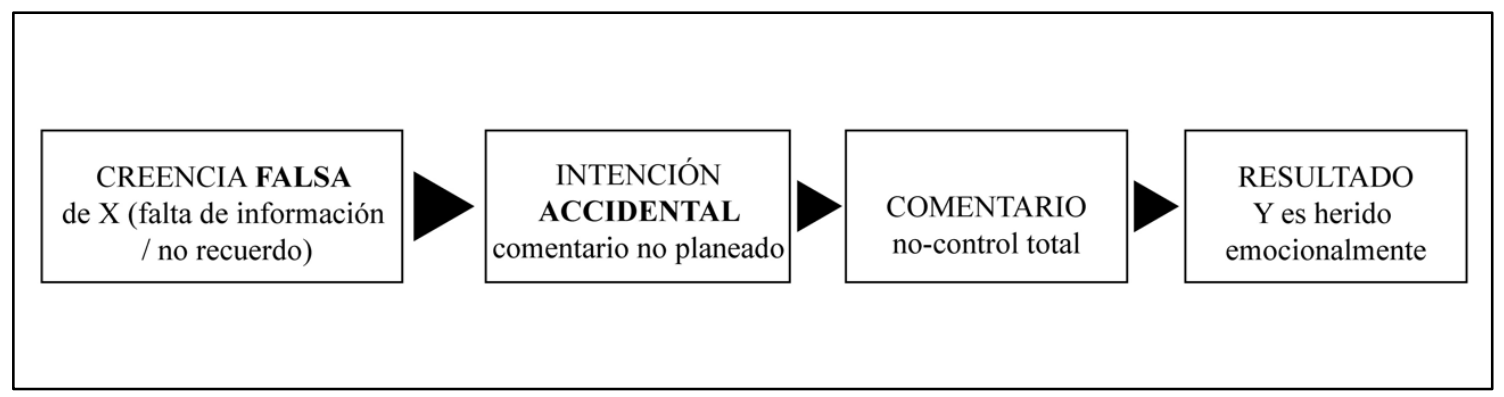

Figura 2-5. Descomposición real de una metedura de pata.

Pero, más allá de entender la metedura de pata desde una perspectiva situacional, es decir, analizando los elementos que forman dicha situación en general, cabría analizar la información presentada desde los diferentes puntos de vista que la comprenden.

Así pues, como se explica a continuación, existirían dos tipos de vías o perspectivas (vía 1 = mente del emisor; vía 2 = mente del observador) por las cuales circulan dos tipos de información muy diferente que conforman la metedura de pata. La primera vía se relacionaría con la información mentalista limitada del protagonista de la metedura de pata, y la segunda vía tendría en cuenta la mente de todos los personajes (normalmente es la información que tiene el observador):

- Vía 1, basada en los pensamientos de Judit: (a) Judit quiere mitigar el padecimiento de Ismael (deseo); (b) el avión se lo regaló alguien ajeno a Ismael (creencia falsa de Judit); (c) Judit hace un comentario para que Ismael no se preocupe (intención deliberada de Judit); (d) Judit realiza dicho comentario (acción controlada); (e) Judit cree que mitiga el padecimiento para que Ismael no se sienta culpable (resultado final falso - resultado que cree Judit).

- Vía 2, basada en lo que un agente externo puede observar: (a) Judit quiere mitigar el padecimiento de Ismael (deseo); (b) el avión se lo regaló Ismael a Judit (creencia 
verdadera de la realidad); (c) Judit hace un comentario ofensivo para Ismael (intención accidental de Judit); (d) Judit realiza el comentario ofensivo sin controlar varios aspectos a tener en cuenta (acción no controlada); (e) Judit hiere a Ismael (resultado final real no deseado por Judit). Ver Figura 2-6. Figura 2-6. Estructura de las dos vías (diferentes perspectivas) en una metedura de pata.

\subsubsection{Las meteduras de pata en el autismo}

Baron-Cohen y su equipo diseñaron en 1999 la tarea de faux pas, hipotetizando acerca de su dificultad para niños y niñas de entre 7 y 11 años (con y sin autismo). En dicho estudio, los niños con autismo - con un cociente intelectual alto o promedio y habiendo resuelto correctamente tareas de primer y segundo orden de TM - encontraron más dificultad en responder correctamente a las preguntas sobre meteduras de pata que los niños del grupo de comparación. Por ejemplo, el grupo de niños con autismo falló en la pregunta sobre detección de la metedura de pata, que hacía referencia a si alguien había dicho algo que no debía haber dicho. En este estudio, se corroboró de esta forma que, aunque los niños con TEA sí que pudieron identificar los estados mentales de los personajes en las tareas de primer y segundo orden administradas en primer lugar, estos mismos niños fallaron en entender la tarea mentalista más compleja de metedura de pata.

En uno de los primeros estudios de réplica en francés, Zalla et al. (2009) usaron la serie de historias de meteduras de pata extraídas del estudio inicial para adultos de Baron-Cohen et al. (1999). En dicho trabajo, se reportó que, en primera instancia, las personas con autismo del estudio identificaron las diferentes meteduras de pata, incluso las 'sobre-identificaron', indicando que algunas historias control también lo eran. Pero, si hay algo que llama la atención de dicho estudio, es la tendencia de los adultos con autismo en juzgar las conductas de los emisores como deliberadas, concretamente, en términos de intenciones malévolas criticables (por ejemplo: estaba celosa, es una mala persona, quería humillarle u ofenderle, es un estafador...). Así pues, los resultados en Zalla et al. (2009), sugerirían que las personas con autismo podrían considerar una metedura de pata como una violación de las normas sociales, no logrando captar su carácter accidental.

Esta dificultad de las personas con autismo en distinguir la intención como deliberada o accidental fue discutida en dicho estudio en referencia a la TM, como prerrequisito necesario para explicar y predecir las acciones ajenas. Sin embargo, Zalla y colaboradores, también destacaron la relación entre intención (reconocer la intencionalidad o accidentalidad de una 
acción) y la moralidad del comportamiento del agente. De hecho, los hallazgos encontrados sugirieron que los juicios de la metedura de pata entendida como más intencionada que accidental estarían motivados principalmente por las consideraciones morales atribuidas por el grupo con autismo del estudio ('era una mala persona'). En resumen, tal y como más tarde se explicó en el estudio de Zalla, Barlassina, Buon y Leboyer (2011), una metedura de pata - y así la entienden las personas NT - sería el subproducto accidental de un acto intencionado basado en creencias falsas; es decir, por definición, se destacaría aquello en lo que las personas con autismo suelen encontrar dificultades.

Para finalizar, en la Figura 2-6 se remarca esquemáticamente la vinculación existente entre los estados mentales y la comprensión correcta de una metedura de pata, y la posible dificultad que subyace a entender todo el conjunto de las dos vías - Vía 1, la del emisor, y Vía 2, la del observador. En resumen, sería:

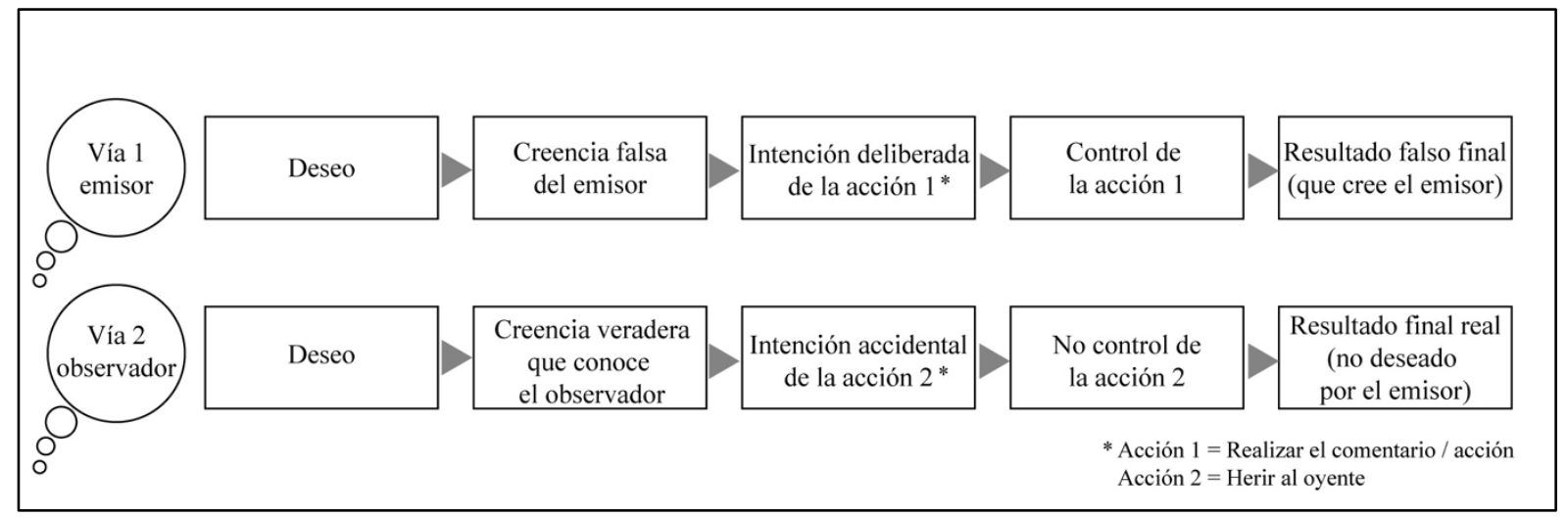

Figura 2-6. Estructura de las dos vías (diferentes perspectivas) en una metedura de pata.

Así, para entender en conjunto la metedura de pata, el observador debería tener cierto pensamiento flexible para deslizarse de una vía a otra (de la información y los estados mentales atribuibles al emisor, a la información que nosotros-observadores poseemos). Sin embargo, como es reconocido, las personas con autismo tienen problemas en flexibilidad cognitiva e inhibición de conducta, pudiendo encontrar de este modo, problemas para integrar la información de ambas vías y tomar una decisión moral basada en la creencia falsa del personaje o de la accidentalidad del acto.

Posiblemente, siguiendo esta teoría, aunque se reconociera que el personaje tiene una creencia falsa de la realidad (vía 1), las personas con autismo podrían encontrar dificultades 
para pasar a la siguiente vía y no reconocer la accidentalidad y el no-control de la acción (vía 2), es decir, se quedarían anclados en la información de una única vía.

Otra explicación que suele estar muy presente a la hora de describir el autismo, pero que no se tiende a aplicar a la discusión acerca de por qué las personas con autismo pueden tener dificultades en integrar los distintos elementos que configuran una metedura de pata, es la teoría de la Coherencia Central Débil (CCD, a partir de aquí). La CCD haría referencia al modo que tienen las personas con autismo de procesar la información, de cómo atienden preferentemente a diferentes elementos de información de forma aislada, en vez de integrar toda esa información en el contexto, lo que sería: entender las partes, pero no el todo (ver Happé y Frith, 2006). Es decir, aplicado a un texto, sería entender las palabras y saber qué significan, pero no encontrar coherencia a las frases ni entender la narración del texto. Trasladando dicha teoría a la comprensión de las meteduras de pata, es posible que las personas con autismo atiendan a pequeños fragmentos de las historias, o a aquello que puedan creer más importante como es el final, sin incidir en pequeños fragmentos que dan coherencia al daño accidental.

Muy posiblemente, además de éstos, existan otros factores o variables que estén influyendo en entender adecuadamente una metedura de pata, y que en la presente tesis se tratarán de determinar, como sería la influencia de la Memoria de Trabajo y el modo en el que los escenarios son presentados (verbales, visuales o mixtos).

Estas hipótesis, arrojan un poco de luz a la peculiaridad de la comprensión total de una metedura de pata que, en nuestro día a día tenemos bastante asumida e integrada, pero que puede resultar sumamente compleja en términos mentalistas cuando existe un déficit en TM.

El trabajo que se presenta, tratará de desentrañar las peculiaridades que subyacen a la comprensión de los estados mentales ajenos y el razonamiento moral de diferentes situaciones ecológicas, las cuales resultan de gran importancia en nuestro día a día. Tan solo hay que observar, durante un recreo, el patio de una escuela para observar las relaciones interpersonales, y también como una de las mayores fuentes de conflicto se debe a la mala atribución de intenciones al compañero (Killen et al., 2011). Ahora, imaginemos que en dicho escenario se encuentran niños y niñas con autismo, los cuales pueden tener problemas en entender la intención del otro. Por este motivo más social, la tesis doctoral que se detalla trata de entender la relación entre TM y moralidad, así como también aportar escenarios realistas dirigidos y administrados a personas con autismo. Su finalidad, es entender qué elementos están influyendo o dificultan entender un conflicto cotidiano en tareas de laboratorio, a sabiendas 
que una mejora en dicha comprensión puede conllevar implicaciones reales en situaciones diarias donde la TM es requerida.

Tras presentar los conceptos básicos del marco teórico y detallados los diferentes estudios centrados en individuos con autismo acerca de TM y moralidad, se presentan en el siguiente capítulo los objetivos de la presente investigación, así como la relación que existe entre estos y cada uno de los artículos realizados. Además, a continuación, se encuentra un resumen en inglés que puede ayudar al lector a remarcar los puntos más importantes de la lectura. 


\subsection{Theoretical Framework Summary}

Theory of Mind (ToM) is critical to evaluate others' intentions, desires and beliefs, and especially to form moral judgements about agents involved in a transgression or accidental situation. As it is well documented, individuals with Autism Spectrum Disorder (ASD) have difficulties in understanding and predicting others' behavior in terms of ToM.

One fundamental aspect of the moral competence is to distinguish between moral and conventional transgressions. This distinction depends on (1) type of action: injustice or rights violation, (2) permissibility of the act, (3) seriousness, (4) authority contingency and (5) generality: general vs local.

Pioneering studies in autism conclude that autistic people succeed in tasks devised to make the distinction between moral and conventional transgression. However, they rely mainly on external factors that could potentially interfere as irrelevant variables (such as crying), or because these moral cases could be presented as simple and unambiguous (negative intent/negative outcome). The central discussion is focused on the link between mental states and moral reasoning.

A person can morally judge another if he / she infers desires and beliefs in the mind of the agent. The concept of the 'intentional-action' explains how mental states cause physical events. Taken all the features together, it could be summarized as, judgements for moral character will depend mostly on people's desires; judgements of moral wrongness will depend on people's actions and mental states; and judgements of punishment will depend on actions, mental states and also outcomes (Cushman, 2015). Due to the problems in inferring mental-states to others, some autistic individuals can attribute moral condemnation mostly based on outcomes rather than intention, and this could represent misunderstandings in attempted harm or accidental situations. Therefore, accidental situations could be considered complex for people with immature ToM. One example of an advanced accidental situation is the so-called faux pas. In the faux pas, understanding another person's false belief has an important role for its correct comprehension. Some evidence suggests that autistic people usually consider intention more deliberated that their comparison groups, assign malevolence to the agent's intention. A novel contribution in the current thesis explains this difficulty regarding faux pas, based on (1) their false belief and intention misinterpretation, or (2) their Weak Central Coherence. Also, working memory and the modality of presentation will be analysed for understanding what variables can affect autistic individuals in the comprehension of a faux pas. 
Capítulo 3 Objetivos de la investigación y Metodología 


\subsection{Objetivos y su relación con los estudios}

En el Marco Teórico, se ha indicado cómo la Teoría de la Mente (TM) juega un rol importante en la comprensión de situaciones sociales, en la toma de decisiones, así como en realizar juicios morales, teniendo siempre en cuenta las diferentes perspectivas y la mente de los agentes que participan en un contexto concreto.

Junto a la definición más teórica de cada apartado y a la explicación más evolutiva de la resolución por parte de las personas con desarrollo neurotípico (NT), se ha incidido en las dificultades que las personas con autismo han denotado en diferentes estudios referidos a los juicios morales y a las meteduras de pata, relacionadas éstas mayoritariamente con sus problemas en TM.

Recientemente, existe un interés relativo a la comprensión de cómo la TM está relacionada con el juicio moral, debido a la comprensión de la intención. Sin embargo, una de las críticas más atribuidas a este tipo de investigaciones es que son pocos los estudios que han contemplado que una misma tarea de TM reúna cuestiones sobre estados mentales (implícita la intención) o moralidad. En esta línea, sería adecuado constatar si las personas con TEA responderían de la misma forma que las personas de su grupo de comparación (NT) en las preguntas relacionadas con intención y moralidad.

Por todo ello, cobra especial sentido la investigación que en esta tesis se presenta, cuyo objetivo general será el de reconocer posibles dificultades en tareas de TM avanzada (transgresiones morales y meteduras de pata) en niños y niñas con autismo, y discutir acerca de qué puede estar influyendo en dicha resolución.

Este objetivo más general, se dividirá en tres objetivos más específicos, que a su vez serán consolidados por sus respectivos sub-objetivos.

Objetivo 1. Discutir las diferentes entre grupos en situaciones deliberadas y accidentales.

1.1. Diferencias intergrupales en situaciones deliberadas - transgresiones morales (Estudio 1)

1.2. Diferencias intergrupales en situaciones accidentales - meteduras de pata (Estudio 2 у 3 ) 
Objetivo 2. Reconocer si ciertos factores pueden tener cierta influencia para la comprensión de las meteduras de pata

2.1. Influencia de la inteligencia (Estudio 2)

2.2. Influencia de la memoria de trabajo (Estudio 3)

2.3. Influencia de la modalidad de presentación de la tarea para el razonamiento complejo mentalista (Estudio 3)

Objetivo 3. Analizar las diferencias entre tareas (Transgresiones Morales y Faux Pas) para los participantes con autismo y NT (Estudio 4).

\subsection{Relación entre objetivos y estudios}

Con el objetivo de reconocer posibles dificultades en las tareas más sociales que implican la comprensión y el razonamiento de los estados mentales que puedan tener las personas con TEA, se han ideado y adaptado una serie de historias que se basan en transgresiones básicas morales, y meteduras de pata. Concretamente, se han diseñado o adaptado 7 historias sobre juicios morales, y 10 historias de meteduras de pata (la mayoría de ellas se pueden encontrar en Garcia-Molina et al., 2016; y cuatro de ellas han sido adaptadas de la tarea de faux pas de Baron-Cohen et al., 1999). Ver Apéndices (7.1; 7.2; 7.3; 7.4 y 7.5). Para una visión más general de cómo se han construido los estudios, ver Figura 3-1(a), y Figura 3-1(b) en inglés.

En respuesta al objetivo 1, todos los estudios consideran un primer apartado de resultados estadísticos que trata de observar si existen diferencias significativas entre grupos en el cómputo total de la tarea o en los sub-apartados que la componen. Aun así, el primer objetivo se respondería detalladamente en el Estudio 1, el cual será analizado en profundidad en el apartado de Discusión de la tesis.

Por lo que se refiere al objetivo 2 , se pregunta, de existir diferencias, ¿son estás debido a la influencia de algún otro factor?, ¿está influyendo la memoria de trabajo, por ejemplo, en la resolución de una metedura de pata? En el mismo sentido, el objetivo 2 trata de averiguar si factores como la inteligencia o la falsa creencia predicen o influyen en la resolución de una metedura de pata, así como si el hecho de presentar dicha tarea en diferentes formatos (visual, verbal o mixto) puede repercutir o influir en una mejor resolución de la tarea. Dicho objetivo se trata en los estudios 2 (inteligencia y falsa creencia) y 3 (memoria de trabajo y modalidades) 
y tiene como fin averiguar qué beneficios puede aportar el control de algunos factores en el diseño de una tarea o intervención en TM avanzada.

El objetivo tercero plantea si existen diferencias entre tareas, es decir, si las personas con autismo (y su grupo comparación) entienden y razonan moralmente mejor una tarea con intención y resultados con valencias no-ambiguas (como las transgresiones morales básicas) que las historias de intención y resultados ambiguos (como las meteduras de pata). Como conclusión final de todos los temas abordados, dicho objetivo se responde en el último artículo (Estudio 4).

\section{ESTRUCTURA \\ CÓMO SE HAN CONSTRUIDO LOS ESTUDIOS}

Reconocer diferencias entre grupos y posibles dificultades;

Determinar posibles influencias en la resolución de tareas de ToM avanzadas

CONCLUSIONES

ESTUDIO 1

\section{MORALIDAD.}

Resultados y conclusiones

Influencia de

- los estados mentales implicados

- la información verbal

- Memoria de Trabajo

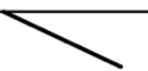

CONCLUSIONES

ESTUDIO 2

\section{METEDURAS DE PATA}

Resultados y conclusiones

Influencia de:

- Falsa Creencia

- Vocabulario

- Forma de procesar la información

De dichos resultados y conclusiones se realizó el Estudio 3 y 4

\section{CONSTRUCCIÓN}

ESTUDIO 3

Basado en:

METEDURAS DE PATA.

Influencia de:

- Presentación tarea (verbal/visual/mixta)

- Memoria de trabajo

- Forma de procesar información
CONSTRUCCIÓN

ESTUDIO 4

Basado en:

MORALIDAD Y METEDURAS DE PATA

Influencia de:

- Estados mentales inplicados

- Moralidad del agente

Figura 3-1(a). Construcción de los cuatro estudios según los objetivos planteados. 


\subsection{Aims, objectives and their relationship with the studies}

The research that is presented in this thesis is based on the general objective which aim is to recognize the possible difficulties that autistic children can have in advanced ToM tasks (specifically, moral transgressions and faux pas), and discuss what may be influencing in their resolution. See Figure 3-1(b) for understanding how the studies have been built.

This general aim is presented as three objectives, which in turn are consolidated by their respective sub-objectives.

Objective 1. Discuss the differences between groups in deliberate and accidental situations.

1.1 Differences between groups in deliberate situations - moral transgressions (Study 1)

1.2 Differences between groups in accidental situations - faux pas (Study 2/3)

Objective 2. Recognise whether certain factors influence the understanding of a faux pas.

2.1 Intelligence influence (Study 2 and 3)

2.2 Working memory influence (Study 3)

2.3 Modality of presentation influence on complex mentalistic reasoning (Study 3)

Objective 3. Analyse the differences between Moral Transgressions and Faux Pas tasks for both autistic and NT participants. 
STRUCTURE

HOW THE STUDIES HAVE BEEN BUILT

CONCLUSIONS

STUDY 1

\section{MORAL TRANSGRESSION}

Conclusions

Influence of:

- mental states

- verbal information

- Working Memory

CONCLUSIONS

STUDY 2

\section{FAUX PAS}

Conclusions

Influence of:

- False Belief

- Vocabulary

- Information processing

First results and conclusions build next studies

\section{CONSTRUCTION}

STUDY 3

Based on:

FAUX PAS

Influence of:

- Modality of presentation (verbal/visual/mix)

- Working Memory

- Information processing

\section{CONSTRUCTION}

STUDY 4

\section{Based on:}

MORAL AND FAUX PAS

Influence of:

- Mental states

- Morality of the agent

Figure 3-1(b). How the studies have been built. 


\subsection{Metodología}

\subsubsection{Participantes}

Un total de 70 participantes, de los cuales 38 tenían un diagnóstico previo al estudio de Trastorno del Espectro Autista (TEA) Nivel 1 de acuerdo con el DSM-5 según uno o más expertos los cuales habían realizado tal diagnóstico. Para su inclusión en el estudio, cada niño / niña debía tener su informe actualizado con fecha no superior a dos años, constando en todos ellos la administración del Autism Diagnostic Observation Schedule (ADOS; Lord, Rutter, DiLavore and Risi, 2001) y Autism Diagnostic Interview-Revised (ADI-R; Rutter, Bailey and Lord, 2003), así como también el Cociente Intelectual (CI) o la posible existencia de comorbilidad con otro tipo de trastornos o daños. En el grupo de comparación, 32 niños con desarrollo neurotípico (NT) fueron elegidos de las mismas clases de los niños con autismo, siendo el requisito sine qua non ser del mismo género que su homólogo/a y una inteligencia promedio. Más tarde, con la administración de las pruebas se constató tal CI. Ver Tabla 3-1 para datos descriptivos.

Los criterios de inclusión fueron:

- Hablar castellano.

- Estar incluidos en su grupo-clase, aunque sean alumnos que asisten determinadas horas a una Unidad de Comunicación y Lenguaje.

- Tener entre 7 y 12 años.

- Obtener una puntuación igual o superior a 80 en la escala Wechsler (CI) - hasta 130.

○ En el Estudio 2, se añadió población con diagnóstico de TEA con un CI promedio-bajo, para así dividir la muestra en este estudio en dos: de 70 - 85; y de $100-120$.

Además, en el grupo de niños con TEA:

- Debían tener un diagnóstico previo de TEA Nivel 1, apareciendo en sus relativos informes la puntuación total en la escala de inteligencia para niños WISC-III (Wechsler, 1991), y el diagnóstico partiendo de la escala ADOS y ADI-R.

Los criterios de exclusión fueron:

- Tener comorbilidad con otro trastorno psiquiátrico, retraso mental o cualquier enfermedad o daño que haya podido afectar al cerebro. 
Tabla 3-1

Datos descriptivos de los grupos por estudio.

\begin{tabular}{lcccccccc}
\hline & \multicolumn{2}{c}{ Estudio 1 } & \multicolumn{2}{c}{ Estudio 2 } & \multicolumn{2}{c}{ Estudio 3 } & \multicolumn{2}{c}{ Estudio 4 } \\
\hline & TEA & NT & TEA 1 & TEA 2 & TEA & NT & TEA & NT \\
\cline { 2 - 9 } $\mathrm{N}$ & 30 & 30 & 18 & 16 & 30 & 30 & 30 & 32 \\
Edad $^{\mathrm{a}}$ & 112.87 & 114.40 & 112.17 & 118.06 & 112.87 & 114.40 & 112.73 & 114.56 \\
$\mathrm{CI}^{\mathrm{b}}$ & 102.83 & 107.03 & 109.56 & 81.10 & 102.83 & 107.03 & 102.83 & 105.75 \\
\hline
\end{tabular}

${ }^{\mathrm{a}} \mathrm{y}^{\mathrm{b}}$ son la media de edad e inteligencia de cada grupo.

\subsubsection{Materiales}

A continuación, se recogen las diferentes tareas que se administraron a los participantes.

En la Tabla 3-2, se encuentra una tabla-resumen de todas ellas clasificadas por estudio.

Cociente de Inteligencia (CI). Para evaluar la inteligencia en los diferentes estudios, se utilizó la adaptación corta de Sattler (1992) de la Escala Wechsler (WISC-III; Wechsler, 1991). Es necesario decir, que la puntuación en la escala Wechsler de los niños y niñas con TEA aparecía en los informes e información los cuales la investigadora pudo tener acceso, con un máximo de dos años de su última administración. Por ello, el proceso a seguir fue:

1. Conocer la puntuación del CI de los participantes con TEA a través del informe realizado por un psicólogo o neurólogo especializado en el que se constataba que el participante tenía un diagnóstico de Trastorno del Espectro Autista según el ADOS y el ADI-R, así como su CI Total.

2. Se administró a la población con TEA la prueba corta de Sattler (1992), la cual garantizó ser un screening fidedigno para el cálculo del CI, tal y como otros estudios ya habían afirmado (Ryan, 1981; Sattler, 2001; Strauss, Sherman y Spreen, 2006).

3. Dados los resultados comparativos, se administró la prueba screening de Sattler (1992) para calcular el CI en la población NT, como medida fiable para medir la inteligencia, con el fin de asegurar un CI comparable en ambos grupos.

Prueba de lo Cubos (WISC-IV; Wechsler, 2005). Como prueba de inteligencia manipulativa se usó el subtest de la escala Wechsler llamada 'Cubos', para todos los participantes, ampliamente administrada para medir la percepción visual, la capacidad de asociar espacialmente y la coordinación visomotriz. Para realizar dicha prueba, se disponía de 9 cubos para formar varios diseños. Los cubos tenían 6 caras, las cuales eran rojas, blancas o 
mitad rojas y mitad blancas. Principalmente, el participante tendría que componer diseños con dichos cubos en 3D, partiendo del dibujo de unas láminas en $2 \mathrm{D}$, sin extralimitarse en su tiempo, o con bonificaciones de tiempo si termina antes.

Prueba de Vocabulario (WISC-IV; Wechsler, 2005). Como prueba de inteligencia verbal se usó el subtest de la escala Wechsler llamada 'Vocabulario', para todos los participantes, ampliamente administrada para medir el nivel de formación de conceptos y el conocimiento de las palabras, así como la capacidad de expresar y explicar el significado de dichas palabras y conceptos. La tarea trataba de presentar al participante una serie de conceptos o palabras para que éste o ésta las explicase lo más precisamente posible.

Memoria de trabajo visual. Se administró la subprueba Memoria Revertida de la escala Leiter-R (Farmer, 2013), la cual mide la capacidad memorística a través de series de dibujos que el sujeto deberá repetir mediante el señalado en el orden inverso del que fue presentado. Por ejemplo, si el instructor señala "jirafa, rana, zapato"; la persona examinada señalará "zapato, rana, jirafa".

Memoria de trabajo verbal. Se administró la subprueba Dígitos de la escala Wechsler (WISC-IV; Wechsler, 2005) utilizando sus dos variantes: dígitos "hacia delante" y dígitos "hacia atrás".

Teoría de la Mente de primer orden: Falsa creencia (FC). Se administró la adaptación de la tarea de FC no-verbal de contenido inesperado basada en las viñetas de De Villiers y De Villiers (2012), a su vez extraída de Schick, De Villiers, De Villiers and Hoffmeister (2007), dónde existía relación entre los personajes (se apreciaba que eran hermanos y el 'objeto' era un animal / mascota), siguiendo el razonamiento de Killen et al. (2011) sobre el contenido social limitado. Ver 7.6 Apéndice 6.

Teoría de la Mente de nivel avanzado. La presentación de las historias de la tarea de metedura de pata y la tarea de las transgresiones morales se realizó mediante el programa multimedia E-prime para ordenador con pantalla táctil, mostrándose dichas historias en orden aleatorio. Las dos tareas de TM de nivel avanzado se podían encontrar en tres formatos: visual, verbal y mixto. Independientemente de su formato, todas se presentaron y sus respuestas fueron 
grabadas a través del programa E-prime (ya fuese como respuesta táctil o como grabación de voz).

Para la construcción de ambas tareas, se analizaron distintos escenarios y estudios similares. Anteriormente a la administración de la muestra total de la presente tesis, se usaron como experimento piloto en población de niños pequeños (ver Garcia-Molina, ClementeEstevan y Andrés-Roqueta, 2014) y en población adolescente con y sin autismo. Además, ambas tareas se tradujeron al inglés y se administraron a participantes ingleses con y $\sin$ autismo (Girard y Garcia-Molina, 2018). De estas primeras lecturas y administraciones, se extrajeron una serie de conclusiones para la mejora de las tareas, como, por ejemplo, no reconducir la respuesta del participante a través de la pregunta. Un ejemplo de reconducción, podría ser: “¿Está bien o está mal que José le tire del pelo a Marcel?”. Para responder a esta pregunta no habría ninguna necesidad de recordar qué ocurrió en la historia. Esta posible influencia en la respuesta fue controlada en las diferentes preguntas, por ejemplo, se preguntó “¿Lo que hizo José, está bien o está mal?”. Además, dentro de lo posible, sin llegar a influir, se utilizaron recordatorios durante las preguntas para que la memoria estuviese mejor controlada. En las historias en las que había viñetas (historias visuales y mixtas), éstas se quedaron permanentes durante las preguntas y respuestas. Por lo que se refiere a las historias verbales, el texto no se situó por escrito delante del participante, sino que durante las preguntas se mostraron algunos recordatorios. Un ejemplo de ello, sería recordar el nombre de los personajes con la voz del personaje a la hora de reconocer quién había hecho o dicho algo malo (ver pregunta 2 y respuestas a continuación).

Las diferentes preguntas se extrajeron de trabajos como el de Banerjee y Watling (2005), Baron-Cohen et al. (1999) y Zalla et al. (2009) para ambas tareas. Además, en referencia a las preguntas sobre moralidad, éstas se basaron en estudios como el de Grant et al. (2005). Aunque cabe recordar que en algún estudio no se utilizó alguna de las preguntas o se añadieron preguntas de respuesta abierta, en general, las preguntas para ambas tareas fueron las siguientes (seguidamente se encuentran las posibles respuestas):

1. Detección del enunciado equívoco en la historia: ¿Alguien dijo algo que no debería haber dicho? [Si/No];

2. Reconocimiento del personaje: En ese caso, ¿quién? [(A)/(B)/Nadie];

3. Emoción provocada: ¿Cómo se habrá sentido (B)? [Bien/Contento; Mal/Triste];

4. Intención del personaje: ¿Tú crees que A quería hacerle sentir así? [Si/No];

5. Moralidad del personaje: ¿(A) es bueno o malo? [Bueno/Malo]; 
6. Moralidad del acto: ¿Lo que hizo (A) está bien o está mal? [Bien/Mal];

7. Ignorancia: ¿Crees que (A) sabía (información que A no tiene/ignorancia)? (Sí/No)* *La pregunta 7, sobre la ignorancia, se utilizó tan solo en la tarea de metedura de pata.

A continuación, se encuentran ambas tareas de TM avanzada analizadas más detalladamente.

Tarea de metedura de pata. Para la creación de los escenarios, se siguió la misma estructura en todas las historias de metedura de pata (ver Figura 3-2).

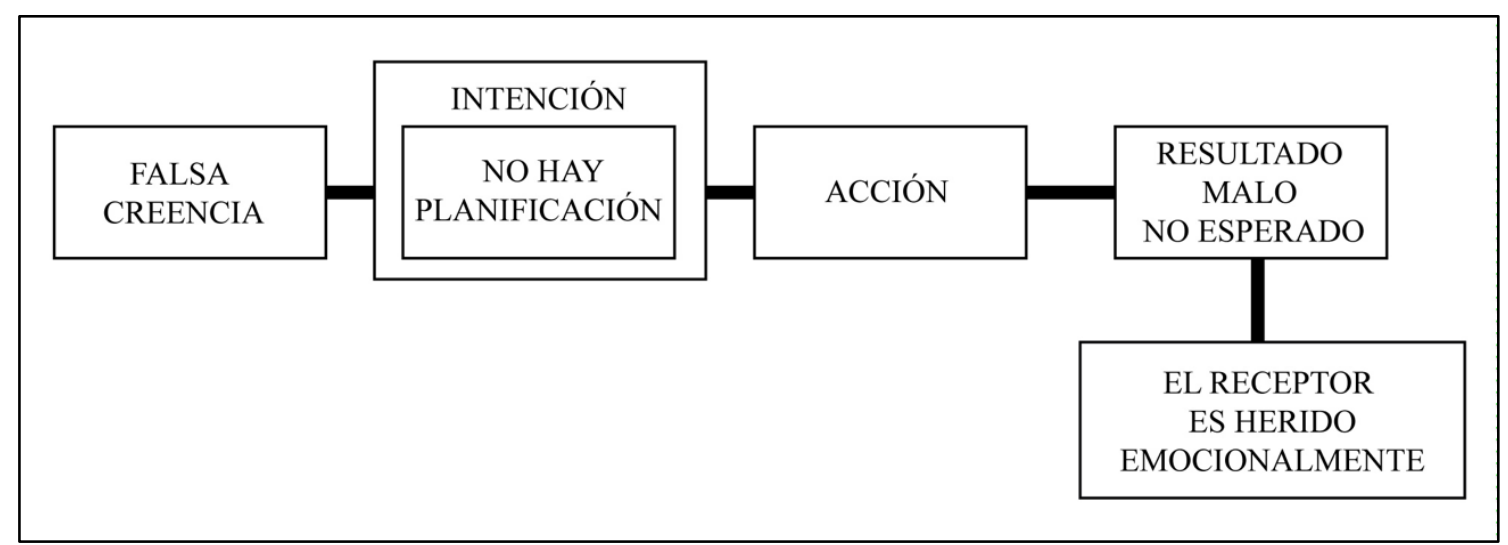

Figura 3-2. Estructura historias de metedura de pata.

Un total de 10 historias fueron administradas, las cuales se pueden dividir por formato de presentación en:

- Tres historias visuales. Viñetas extraídas de Garcia-Molina et al. (2016). Consistían en dos viñetas simples recreando una situación embarazosa o de error. Sus preguntas aparecieron en pantalla ( $\sin$ audio) y sus respuestas fueron visuales. Ver las historias visuales en el 7.1 Apéndice 1, y ejemplo de pregunta y respuesta en la Figura 3-3. 


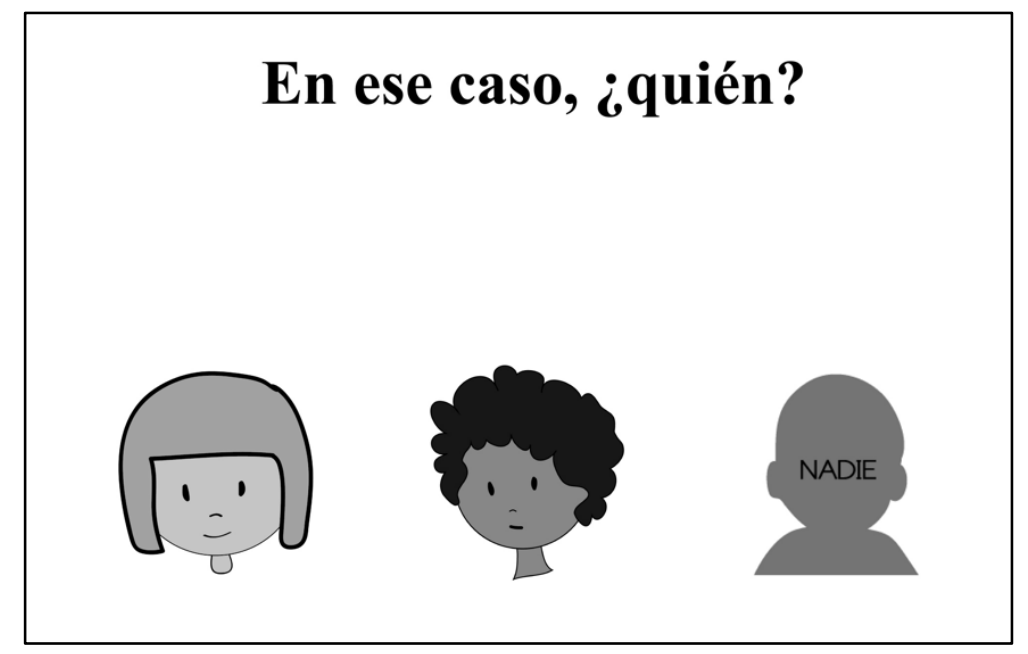

Figura 3-3. Ejemplo de respuesta visual.

- Tres historias verbales. Creadas expresamente para este trabajo, siguiendo el patrón de la tarea de faux pas de Baron-Cohen et al. (1999). Consistían en un audio que recreaba una situación mediante diálogos y narración (se usaron diferentes voces bien diferenciadas). Sus preguntas se realizaron a través del audio (no escritas) y sus respuestas fueron verbales (el escolar respondía en voz alta a las preguntas y éstas fueron grabadas por el programa). Ver historias en 7.2 Apéndice 2.

- Cuatro historias mixtas. Viñetas extraídas de Garcia-Molina et al. (2016), basadas en la tarea de faux pas de Baron-Cohen et al. (1999). Consistían en viñetas simples (muy poco distractores y sin fondo), narración escrita y audio que recreaba simultáneamente la situación completa. Sus preguntas aparecieron en pantalla (y con audio) y sus respuestas fueron visuales (muy similares a las de las historias visuales). Ver historias en 7.3 Apéndice 3.

Un total de 7 historias fueron administradas, las cuales se pueden dividir por formato de presentación en:

Tarea de transgresiones morales. Para la creación de los escenarios, se siguió la misma estructura, basada en el modelo de Cushman (2015), en todas las historias de transgresiones morales, en inglés BBB; Bad desire - Bad intention - Bad outcome (Figura $3-4)$. 
- Tres historias verbales. Creadas expresamente para este trabajo, basadas en situaciones ecológicas habituales entre escolares y siguiendo el patrón del modelo explicado de Cushman (2015). Consistían en un audio que recreaba una situación mediante diálogos y narración (se usaron diferentes voces bien diferenciadas). Sus preguntas se realizaron a través del audio (no escritas) y sus respuestas fueron verbales (el escolar respondía en voz alta a las preguntas y éstas fueron grabadas por el programa). Ver historias en 7.4 Apéndice 4.

- Cuatro historias mixtas. Viñetas extraídas de Garcia-Molina et al. (2016). Consistían en viñetas, narración escrita y audio que recreaba simultáneamente la situación completa. Sus preguntas aparecieron en pantalla (y con audio) y sus respuestas fueron visuales (muy similares a las de las historias visuales). Ver historias en 7.5 Apéndice 5.

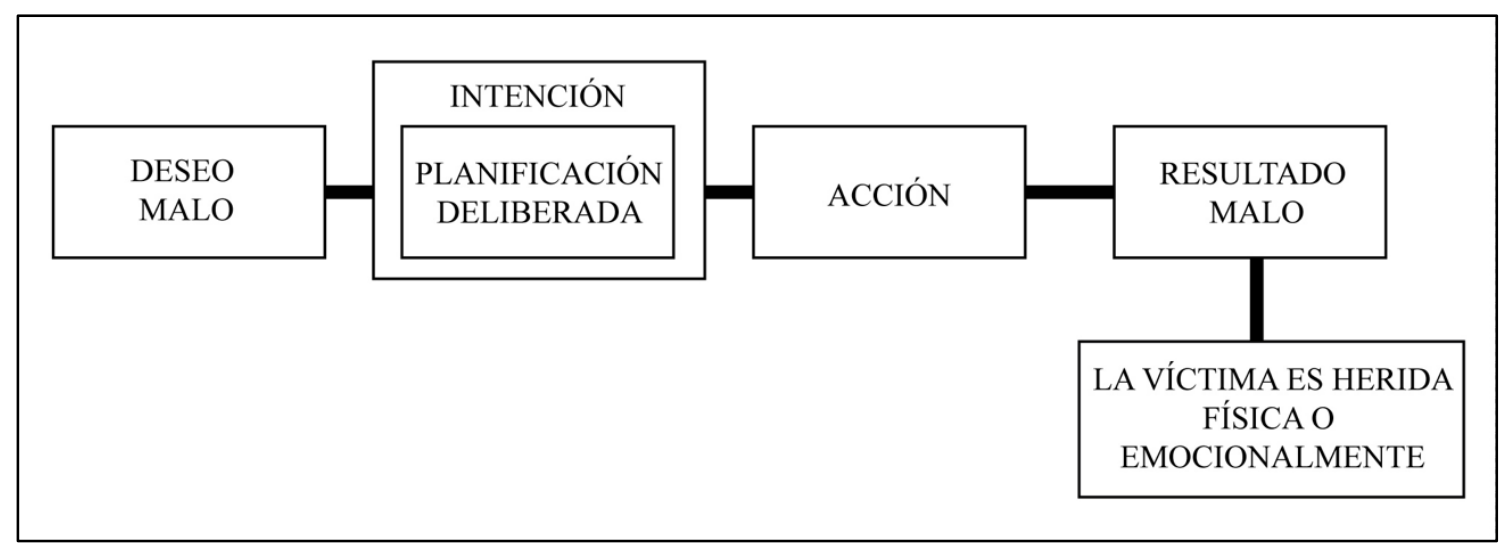

Figura 3-4. Estructura historias de transgresión moral.

Para una visión más general, se presentan en la Tabla 3-2 las tareas que se han administrado en cada uno de los estudios que comprende esta tesis. 
Tabla 3-2

Tareas administradas en cada estudio

\begin{tabular}{|c|c|c|c|}
\hline Estudio 1 & Estudio 2 & Estudio 3 & Estudio 4 \\
\hline CI. Adaptación corta & CI. Adaptación corta & CI. Adaptación corta & CI. Adaptación corta \\
\hline Wechsler (Sattler). & Wechsler (Sattler). & Wechsler (Sattler). & Wechsler (Sattler). \\
\hline $\begin{array}{l}\text { Tarea de creencia } \\
\text { falsa de primer orden } \\
\text { de TM. }\end{array}$ & $\begin{array}{l}\text { Tarea de creencia } \\
\text { falsa de primer orden } \\
\text { de TM. }\end{array}$ & $\begin{array}{l}\text { Tarea de creencia } \\
\text { falsa de primer orden } \\
\text { de TM. }\end{array}$ & $\begin{array}{l}\text { Transgresiones } \\
\text { morales mixtas. }\end{array}$ \\
\hline \multirow[t]{5}{*}{$\begin{array}{l}\text { Transgresiones } \\
\text { morales verbales. }\end{array}$} & $\begin{array}{l}\text { Tarea Cubos } \\
\text { (WISC-IV). }\end{array}$ & $\begin{array}{l}\text { Memoria de Trabajo } \\
\text { visual (Leiter-R). }\end{array}$ & $\begin{array}{l}\text { Meteduras de pata } \\
\text { mixtas. }\end{array}$ \\
\hline & $\begin{array}{l}\text { Tarea Vocabulario } \\
\text { (WISC-IV). }\end{array}$ & $\begin{array}{l}\text { Memoria de Trabajo } \\
\text { verbal (WISC-IV). }\end{array}$ & \\
\hline & $\begin{array}{l}\text { Meteduras de pata } \\
\text { mixtas. }\end{array}$ & $\begin{array}{l}\text { Meteduras de pata } \\
\text { visuales. }\end{array}$ & \\
\hline & & $\begin{array}{l}\text { Meteduras de pata } \\
\text { verbales. }\end{array}$ & \\
\hline & & $\begin{array}{l}\text { Meteduras de pata } \\
\text { mixtas. }\end{array}$ & \\
\hline
\end{tabular}

\subsubsection{Procedimiento}

\subsubsection{Creación y elección de las tareas}

Revisada la literatura reciente sobre la temática que en esta tesis se plantea, en el 2013 se solicitó el material necesario al servicio de la Docimoteca de la Universitat Jaume I (WISCIII; WISC-IV y Leiter-R), y se creó la primera versión de la tarea de Transgresión Moral y la adaptación y creación de la tarea de Faux Pas. Para esta última adaptación, Baron-Cohen aprobó previamente que dichas historias se adaptaran y se ilustraran.

Las diferentes historias de redactaron, ilustraron (Adobe Illustrator), grabaron (Adobe Audition), y montaron para administrarlas a través del programa E-Prime. Cuando esta primera versión estuvo completa, se administró a diferentes participantes adultos y niños sin un diagnóstico de autismo para observar posibles fallos. Partiendo de las conclusiones de dicho primer estudio piloto (Garcia-Molina et al., 2014) se revisó la tarea, y finalmente, se administró la versión final a un grupo de adolescentes con autismo, los resultados de los cuales no se incluyeron en la muestra total de esta tesis por su edad (entre 14 y 17 años). 


\subsubsection{Permisos y Recogida de datos}

La investigación que a continuación se detalla fue previamente aprobada por las autoridades pertinentes (Generalitat Valenciana), la Comisión Deontológica de la Universitat Jaume I, las escuelas y los centros, los maestros, profesionales y las familias de los participantes (ver cartas modelo en 7.0 Apéndice 0). De las reuniones concretadas con los directores, psicólogos y orientadores de los centros, un total de 6 escuelas públicas de la Comunidad Valenciana participaron en el estudio. Además, también tuvimos acceso a un centro de neuropsicología donde los participantes habían sido diagnosticados para comprobar puntajes en ADOS y ADI-R, así como sus CI.

Durante meses, las tareas fueron administradas a los participantes en una sala tranquila, sin distractores, con una mesa y dos sillas. Se programaron dos sesiones de evaluación, cada una de una duración aproximada de entre 45 y 60 minutos.

Siguiendo el criterio de inclusión para los estudios explicado en el apartado de la Muestra (CI y pasar una tarea de falsa creencia de primer orden), se administraron posteriormente las tareas de TM avanzada. En cada estudio se puede encontrar detalladamente cuántos participantes se incluyeron para su investigación más concreta (ver apartado de Procedimiento de cada estudio). Después de la administración de las pruebas, se hizo un informe concreto de cada participante dirigido a familias y maestros, que mostraba las puntuaciones, implicaciones y recomendaciones detalladas para cada niño y niña. 
Capítulo 4 Estudios / Chapter 4 Studies 


\subsection{Study 1 \\ Moral Judgements in Autism. A Novel Moral Judgement task}

Garcia-Molina, I., Clemente-Estevan, R.A. \& Andrés-Roqueta, C. (UNDER REVIEW). Moral Judgements in Autism. A novel moral judgement task. Cognitive Development. 


\begin{abstract}
This study compared the theory of mind features of moral judgements in 60 children with and without Autism Spectrum Disorder (ASD), using a novel verbal moral judgement task. The task focused on three different scenarios which were morally unacceptable. Five measures were assessed with forced-choice responses, whilst the other two were categorised according to the quality of the response (categories: (i) related to mental states; (ii) descriptions; (iii) literal reiterations; (iv) inappropriate). No significant differences regarding the forced-choice answers were found between the groups. Justifications were classified mostly in category (ii) description by the ASD group, whereas the justifications of neurotypical children referred to the use of mental-state words and descriptions for explanations. Implications of ToM in moral reasoning for children with ASD are discussed.

Keywords: moral judgements; theory of mind; morality; intention; autism.
\end{abstract}


Human-beings tend to read others' actions and intentions in order to understand social interactions and communication (Astington, Harris and Olson, 1988). Importantly, based on what we predict from a specific behaviour, we can form a judgement and decide to act accordingly. For example, if we recognise that a child hit someone by accident, the moral evaluation we might make of that child will be different from that formed if we recognise that the action was carried out on purpose. In this recognition, the capacity that allows us to attribute mental states (such as desires and beliefs) to oneself and to others plays a central role and is known as Theory of Mind (ToM; see Frith and Frith, 2005; Hughes and Leekam, 2004, or Perner, 1999, for a review). Therefore, ToM ability is critical when evaluating others' intentions and, as in the example given, it is important to make a distinction between intentional and non-intentional actions of an agent. In consequence, ToM enables us to form a moral judgement about the agent involved in a specific situation (Buon et al., 2013; Turiel, 2006; Wellman and Liu, 2004). Indeed, there is a link between ToM reasoning and moral judgement that runs from the intention of an action to the moral evaluation (Leslie, Knobe and Cohen, 2006). Therefore, the elemental features involved in an intentional action should be analysed, according to Cushman (2015), in order to better understand: (i) mental states - beliefs and desires: the agent has a mind and establishes a goal; (ii) intention - deliberate: the agent may make a plan to obtain his/her goal; (iii) control of the action: the agent carries out the actions included in that plan; and (iv) outcome: that action triggers an outcome or consequence. Thus, to evaluate the morality of the agent, the observer should correctly infer the agent's desires (whether they are prosocial or antisocial), beliefs (whether the agent has true or false beliefs according to reality) and the agent's intention (whether the act was carried out on purpose or accidentally) and, finally, use this information to decide whether the agent is a good or bad person, or even his/her innocence or guilt in the event. According to this model, the moral evaluation of the agent would be in some cases independent of the outcome. For example, we 
could imagine this situation: Mark wants to kill a bird and he has the deliberate intention of doing it, but finally, he failed to do it. Mark will be judged as a "bad person" for the mere presence of a malicious desire (regardless of whether the bird does not die), true belief (he knows he can kill the bird using, for example, a stone) and his deliberate intention of doing this action, which speaks more strongly against his morality than the final outcome.

Thus, it seems that, first, the moral judgements that a person makes about another agent will depend mostly on the inferences made about the agent's desires, beliefs and intention, and not only on the outcome of his/her action (as in the case of the bird). Second, another judgement can be contemplated in the bird situation: Judgements of moral wrongness, which will depend on the agent's mental states and also the agent's action, i.e., if the child kills the bird on purpose, this will be wrong because of the action itself, but also because the child planned it deliberately. Finally, judgments concerning punishment will depend not only on actions and mental states, but also on the outcome caused by his/her actions (in this case, laws make no provision for his/her punishment) (Cushman, 2015).

Therefore, the mature development of the capacity for understanding mental states will be important for adequate moral reasoning. For this reason, younger children between 3 and 4 years of age usually focus more on the outcome (e.g., the child hit his sister and now she is crying), and less on the intent or the mental states of the agent (e.g., he did not mean any harm or he did not do it on purpose...). See Cushman et al. (2013) and Margoni and Surian (2017), for more information.

\section{Moral judgements in autism}

It is well established that autistic people have difficulties in ToM (see the classic studies of Baron-Cohen, Jolliffe, Mortimore and Robertson, 1997; or Happé, 1994a). These difficulties to use the mental information about agents' desires and beliefs can directly affect moral 
judgements, and for this reason it appears relevant to assess whether the ability of autistic people to understand morality is consequently impaired. The literature on moral judgements in autism suggests two significant findings (see Margoni and Surian, 2016). First, it has been proposed that autistic people exhibit difficulties when making moral judgements in terms of intention due to their impairment in understanding mental states (Moran et al., 2011; Zalla et al., 2009). Second, it has been observed that they appear to develop a basic moral judgement (i.e., a bad outcome produced by a bad intention), probably because they rely on evident clues such as the transgression outcomes or the emotions of the victim (Blair, 1996; Rogers, Viding, Blair, Frith and Happé, 2006; Shulman, Guberman, Shiling and Bauminger, 2012; Weisberg and Leslie, 2012; Zalla et al., 2011). Even so, it is important to take into consideration that autistic individuals could face difficulties in recognising emotions and this may, in turn, affect moral decisions. This could be caused by comorbid alexithymia (impairments in identifying one's own emotional responses), associated with approximately $50 \%$ of the ASD population (see Brewer et al., 2015; Patil, Melsbach, Hennig-Fast and Silani, 2016).

\section{Moral judgement tasks and ToM tasks}

In general, studies often use the administration of two separate tasks to assess ToM and moral judgements. In this regard, few studies contemplate a measure that involves both ToM and moral judgement evaluations in the same task. While moral judgement tasks cover a wide range of measures across studies (distinguishing moral from conventional transgressions, punishment acceptability, ratings of severity, or desires to acquire something that has been prohibited by someone, and so forth), prior research on moral judgements and ToM has used first- or second- order false belief (FB) tasks, which are administered to younger children and are based on the inferences of beliefs and not on intentions or social/affective aspects (see Killen et al., 2011). 
For example, Blair (1996) pioneered moral judgement research in autism in comparison to mild learning difficulty (MLD) and neurotypical (NT) by distinguishing moral transgression (e.g., hitting someone) from conventional transgression (e.g., talking in class). Autistic participants (age range $=8-17$ years old) were divided into Non-ToM and ToM groups, depending on whether they passed the first-order FB task successfully or not. Their findings showed that the group of autistic children who lacked the ability to mentalise were sensitive to the distress of others. Autistic children made the distinction between moral and conventional transgression. Moreover, their results in the false belief tasks were not associated with the tendency towards this distinction. Later, following Blair's study, Leslie, Mallon and DiCorcia (2006) also compared autistic children ( 7 to 16 years old; mean verbal mental age of 5 years and 11 months) to NT children ( 3 to 5 years old) on basic moral judgements (basic distinction between good and bad, avoiding to test the comprehension of intention) and the punishment or reward they deserved. The authors found that only one autistic participant successfully passed the two standard first-order FB tasks. However, the ASD group understood how bad or good the acts were (item example: 'Was it good, bad, or just okay that Patty hit Sarah?' [good, OK, bad]). Hence, both studies found that autistic children were as good as NT children at distinguishing morally acceptable acts from morally unacceptable acts. Thus, the ASD groups in these two studies would be capable of reaching a basic understanding of right and wrong moral aspects.

It could therefore be seen that moral judgements would be relatively independent of ToM, thereby showing that autistic children who failed standard FB tasks may yet retain a basic sense of morality. However, in both papers the authors argued that a minimal level of ToM would be necessary to understand some transgressions, such as the mental states involved in the act of lying. Telling a lie requires that the liar deliberately and successfully creates a FB in the mind of another person. Thus, ToM is an important tool to enable the agent to do so but is also a 
valuable aid enabling the observer to fully understand the transgression (Talwar and Lee, 2008). As a highlighted point, the two studies cited above were based on a basic distinction of good or bad in stories with both positive and negative valences, and they did not ask children about the agents' intentions, nor were the participants required to deploy ToM to make moral distinctions.

Importantly, a working hypothesis formulated in Margoni and Surian's work (2016) paves the way to understanding that when tasks are simple with unambiguous moral cases (i.e., negative outcome produced by an intentional action), autistic children can respond as well as NT children. Therefore, when the moral judgement tasks involve ToM competence, differences can appear and reveal difficulties in reasoning about others' mental states when autistic people are able to pass first- or second-order classical FB tests.

Some studies centred on advanced ToM tests (complex scenarios where an advanced level of ToM is required) have recently shown that autistic individuals have difficulties in distinguishing the intentionality of the actions (accidental/deliberate) and these misunderstandings could be affecting their moral judgements. In their study, Moran et al. (2011) reported a mismatch between the comprehension of the negative outcome of the agent's action and his/her actual intention (good / accidental), and it was mediated by the ToM impairments of the ASD group. Specifically, as a consequence of the ToM deficit, autistic participants blamed the agent who caused the accidental harm more severely than the NT group did. However, no differences between groups were found in the moral judgements of neutral acts, attempted harm or intentional harm.

In another study based on an accidental action task (Zalla et al., 2009), the so-called faux pas test (Baron-Cohen, O'Riordan, Jones, Stone and Plaisted, 1999), the interpretations of blunders or faux pas were analysed. A faux pas occurs due to a FB of the real situation, when the speaker says something that can hurt the listener emotionally, although this negative 
emotional impact is not intentional. Zalla and colleagues (2009) observed that adults with ASD could correctly detect the faux pas, but they failed to interpret the FB of the speaker. They also provided explanations in terms of malevolence, judging the speaker's intention to humiliate or offend the listener as deliberate (intentional).

Consequently, the studies cited above may indicate the idea that autistic individuals do have difficulties in understanding intentions in terms of ToM reasoning when the situations are socially complex (or ambiguous, as pointed out by Margoni and Surian, 2016). This probably occurs because they tend to judge the culpability of the agent on the basis of the explicit observation of his/her outcome (hurting a person) rather than his/her implicit intention; that is, they judge the agent as "bad" because the outcome was bad or harmful, regardless of whether the agent's intention of his/her action (Zalla and Leboyer, 2011).

Another study which asked whether autistic children were able to judge transgressions in terms of outcomes or intentions was that of Grant et al. (2005). Grant and her team (2005) presented pairs of vignettes adapted from Elkind and Dabek (1977), in which the actions were either deliberate or accidental and caused a bad outcome (harm to a person or to an object). Results showed that autistic children used information about intention as the basis of culpability judgements, considering the agent's intention to be more relevant than the consequences for the moral evaluation. The members of the ASD group were also capable of judging the negative consequences as worse (i.e., as more culpable) when a person, rather than an object, was involved. Nevertheless, results differed when the participants were asked to verbally justify their responses. Grant and colleagues classified the verbal responses of this study into categories, giving importance to the pain (i.e., greater culpability in the case of injury to persons than damage to property or objects), reversibility (i.e., property can be replaced, but damage to another person cannot) and intent/motive (whether an act was deliberate or accidental). Although autistic children could offer some appropriate verbal justifications about 
moral judgement of the agent, their explanations were poorer, as they usually reiterated the story rather than elaborating on their explanations regarding the culpability, with fewer references to the agent's intention. The majority of the justifications in Grant's study were classified as 'not scorable'. These findings are related to the study conducted by Shulman et al. (2012), in which the authors found that NT children used significantly more abstract rules as rationales than autistic participants and, in addition, autistic children provided more nonspecific condemnations of the behaviours and poorer explanations, i.e., "that's bad" or "you can't do that". Authors argued that high scores (of appropriate answers) were linked to the use of mental state words in the children's explanations (e.g., Bishop and Norbury, 2002).

\section{Present study: a novel moral judgement and ToM task}

In the current study, we investigate whether or not autistic individuals are able to use relevant implicit information about intention to form their moral reasoning. Indeed, although autistic participants could have problems in moral tasks which require more complex mentalising abilities, the studies analysed showed no differences between NT and ASD groups when responses were forced-choice (i.e., good/bad; see Leslie et al., 2006) and when tasks were not ambiguous (Margoni and Surian, 2016). Instead, verbal justifications have played an important role in showing the problems that those with ASD have in dealing with intentions (in terms of mental states and plans) and morality (act-wrongness or agent-culpability), even in basic moral stories. This importance of verbal information (justifications) remains to be further explored, specifically in the justifications made about the intentions and morality of the agents' actions in different moral scenarios with intentional actions (accidental actions, as in the faux pas task, are not considered in this study due to their complexity and ambiguous features). 
Our approach is innovative in two main ways. First, the present study introduces a novel moral task based on understanding moral stories which involve both ToM competence and moral judgement evaluations (as argued in the study by Killen and colleagues, 2011) with both types of questions: open-ended and also with dichotomous answers. Second, our dichotomous questions were different from those used in the other studies mentioned (such as Blair, 1996; Leslie et al., 2006) in that they were intended to ask a general question rather than being specific and repeating the act or the transgression (e.g., Was what [the agent] did right or wrong?" versus "Was it bad for Catherine to pull Sally's hair?"). As in the study by Nobes, Panagiotaki and Bartholomew (2016), this information was excluded here because it provided the answer to the wrongness question, and thus, could increase children's tendency to make outcomebased judgments in their reasoning. Other questions were also added (i.e., emotions triggered in the victim - such as the resulting harm). In addition, as commented on in Leslie et al. (2006), different actions were contemplated in terms of mental states involved in some transgression (i.e., it is more necessary to appeal to mental states in the act of 'lying' than in the act of 'breaking' an object). Finally, open-ended questions were based on morality and intention in order to examine the importance of the mental states involved in moral judgement stories (as in Grant et al., 2005).

Therefore, the goal of the current study was to compare ASD and NT groups in terms of their comprehension of the moral stories which include complex ToM events. To address this goal, some research questions will be answered.

Related to the forced-choice answers:

1. Are there any differences between autistic and NT children in the forced-choice answers to the moral stories regarding (1) detection of the transgression, (2) identification 
of the perpetrator who caused the bad action, (3) emotion triggered in the victim, (4) perpetrator's morality, and (5) wrongness of the act?

2. a) Are there any differences in the forced-choice answers to the stories depending on the mental states involved in the transgression (for example: when the bad action is 'lying' to someone, the story could be more difficult to understand than when the action is more basic, such as 'breaking' an object)?

b) Do these differences exist in the ASD group?

c) Do these differences exist in the NT group?

Related to verbal justifications:

3. Are there any differences between autistic and NT children in action-morality and intention justifications?

4. Which justifications (classified in categories) are more reiterative in the two groups?

5. Are there any differences between autistic and NT children in their justifications based on ToM and no-ToM content?

\section{Predictions}

Related to forced-choice answers:

The first hypothesis of the present study was that autistic and NT children will not differ in their choice answers in the moral judgements task (as per Blair, 1999, and Leslie et al., 2006) since the transgressions contemplated in the study are based on unambiguous moral transgressions (bad intention - bad outcome; following Margoni and Surian's hypothesis).

The second hypothesis was that autistic children should find it more difficult (and differences are expected between stories) to understand when the agent's action involved mental states than when the action is more basic (as commented in Leslie et al., 2006). No differences between stories are expected for the NT group. 
Related to verbal justifications:

The third hypothesis was that differences could appear between groups in their verbal justifications about intention and action-morality questions, due to the expectation that autistic children would use less ToM terms than NT children. Difficulties and poor explanations are expected in the ASD group's justifications, with the most of the classifications in reiterations or nonsensical justifications (as in Grant et al., 2005, and Shulman et al., 2012).

\section{Method}

\section{Participants}

A priori sample size analyses using $\mathrm{G}^{*}$ Power indicated that 27 participants per group would be adequate for t-tests $(d=.80 ; \alpha=.05$; desired level of assurance $=.08)$; and a total sample size of 52 participants would be adequate for $\chi^{2}$ tests $(d=.80 ; \alpha=.05$; effect size $w=.05)$. This sample was comparable to those used in previous research in this field (i.e., Shulman et al., 2012). A total of 63 children took part in the present study, three of whom did not pass Task 1 (first-order false belief task) and were excluded, thus leaving 60 final participants (see Procedure section). All the participants were native Spanish speakers and attended ordinary schools in the Valencian Community (Spain) in an inclusive setting. Thirty children had been diagnosed with level 1 Autism Spectrum Disorder (26 boys, 4 girls). Their ages ranged between 7 and 12 years old (mean $=112.87$ months, $\mathrm{SD}=18.58$, age range: $88-145$ months) and they all had a typical IQ range $($ mean $=102.83, \mathrm{SD}=14.23$, IQ range: $80-130)$. Each child had been diagnosed with autism by a qualified paediatric neurologist or psychologist prior to taking part, using the Autism Diagnostic Interview-revised (Rutter, Bailey and Lord, 2003) and the Autism Diagnostic Observation Schedule (Lord, Rutter, DiLavore and Risi, 2001). At the time of the study, all the autistic children were attending ordinary schools and receiving specific intervention from a speech therapist at school. In addition, the autistic students were also 
receiving specialist support from a specialised neuropsychological intervention centre at that time.

The autistic children were compared to 30 NT children on the basis of gender ( 26 boys, 4 girls) and age $($ mean $=114.40$ months, $\mathrm{SD}=19.11$, age range: $84-145$ months; $t(58)=.32, p=.75$, $d=0.11$ ) and no differences were found between the groups in terms of IQ (mean $=107.03$, $\mathrm{SD}=12.02$, IQ range: $80-130 ; t(58)=1.23, p=.22, d=0.32)$, measured with Sattler's $(1992)$ short version of the Wechsler scale (WISC-III).

\section{Materials}

All tasks were administered and performed in Spanish, since all the participants speak Spanish as their mother language.

\section{Intelligence Quotient (IQ):}

Sattler's short adaptation (1992) of the WISC-III (Wechsler intelligence scale for children (Wechsler, 1991) was administered first (in the NT group). The WISC-III full scale IQ was highly correlated with the short form (Vocabulary and Block Design) as found in classic studies (Ryan, 1981; Sattler, 1992). Comparison of scores was possible as all the participants with autism had diagnostic reports made by a qualified psychologist or neurologist at their specialist neuropsychology centre within the previous two years. Given this high correlation between the short form and the full scale of the WISC-III, the short form WISC-III was administered to the NT group as a reliable estimate of the group's intelligence quotient, with the main objective of ensuring comparable IQ levels in both groups.

\section{Theory of Mind tasks:}

Task 1: ToM first-order false belief task. All participants were screened by means of a ToM first-order FB task adapted and designed from the study by De Villiers and De Villiers in 2012 (adapted from Schick et al, 2007). This false-belief task was designed as a sequence of 
pictures, where the first character (a girl) places an unusual object (a little chick) into a box originally containing shoes. The children are then asked which object the second character (a boy) thinks will be inside the box (by presenting the option of a chick or shoes). Specifically, they were asked: "What goes in here?", "What is he thinking?" and "What is actually inside the box?". The maximum score was required (3 points, 1 point for each question).

Task 2: Moral Transgression (MT). The MT task was a novel task created by the authors for this study. The moral scenarios (lying, stealing, physical and emotional harm to someone else) were based on universal moral norms (regardless of the authority or social context, which involve a victim and are general in scope) (see Sousa, 2009). Following Cushman's model (2015), the "structure" of the stories was unambiguous: bad (or selfish) desires - deliberate intention - wrong action - bad outcome. Based on this structure, three different basic moral stories were created for the purpose of the study:

1. Dog story. The transgression involves a lie and inculpating an innocent: the child's desire was not to be punished and she deliberately blamed the dog, which cannot defend itself.

2. Car story. The transgression involves breaking a valuable object which belongs to someone else: the boy's desire was to reach his destination as quickly as possible and he deliberately broke the window of his friend's car to get the keys.

3. Sandwich story. The transgression involves stealing or doing something without permission: the man's desire was to satisfy his hunger and he deliberately ate his friend's sandwich without permission.

Information was collected through dichotomous choice answers and verbal justifications and was subsequently categorised (see Questions section).

For better comprehension, the three stories are placed in the 7.4 Appendix 4. 


\section{Questions}

After each story, five questions were asked that required forced-choice responses (correct answers are in bold): (1) detection of the transgression (Did someone do something that they should not have done? [Yes/No]), (2) identification of the perpetrator who caused the bad action (If so, who? [perpetrator/victim/nobody]), (3) emotions triggered in the victim (How does [name of the victim] feel? [feeling happy/feeling sad), (4) perpetrator morality (Is [the perpetrator] good or bad? [good or bad]) and (5) wrongness of the act (Was what [the perpetrator] did right or wrong? [right/wrong]). In this last question (5), all participants chose the correct response ('it was wrong'), and it was not included in the analysis; however, this result is commented on in the discussion. Therefore, the score range per story was $0-4$, excluding the point for the fifth question, and the score range of each variable (e.g., total score of (1) detection of the transgression) was $0-3$.

In addition, two other open-ended questions were asked to obtain more verbal information: (6) action-morality: 'Why was what [the perpetrator] did wrong?' and (7) intention: 'Why did the character do this? What was his/her intention?'. The answers to both questions were classified into four categories according to: (i) appeal to mental states - especially desires - that are involved in the transgression; (ii) superficial description - just the description of the action; (iii) literal reiterations - the child copies the same sentence from a character in the story; and (iv) inappropriate or nonsensical - the child provides an inappropriate justification which does not fit into any of the categories.

For analysis, the mean value was understood as useful in interpreting participant comprehension in the descriptive analysis and its subsequent comparative analysis of the categories (for example in Table 4-4). Thus, the scores were distributed in relation to the adequacy of the answers on an inverse scale: score of $4(\max )=$ category (i) justification, down to a score of $1(\min )=$ category (iv) justification. A score of 0 was given for a non-response, 
since one child in the ASD group did not reply to the justification questions. These scores did not represent a linear or ordinal scale from low to high ToM content. Instead, the scale represents the complexity of the explanations classified from low to high. Scores from $0-3$ essentially reflect no ToM content in the justifications, while scores of 4 indicate ToM content. For this reason, an additional score was added based on the analysis of the above-mentioned categories: $0=$ no-ToM content category (no reponse or categories iv, iii, ii), and $1=$ ToM content category (category i).

\section{Procedure}

This study was approved by the research ethics committee of Valencian Community, 'Universitat Jaume I' university and the school authorities. Prior to taking part in the study, the parents of each child gave informed consent for their children to participate. As a result of these meetings, six mainstream centres all from the Valencian Community participated in the study. Over several months, the different tasks were administered to the children, including the short Sattler adaptation (1992) of the WISC-III, the ToM task adapted from De Villiers and De Villiers (2012) and the verbal moral task.

Each task was performed in a quiet room free of any distractors with a table and two chairs. The duration of the session was approximately 50 minutes per participant and only children who passed the ToM first-order FB task (Task 1) went on to the additional MT task (Task 2). Three children did not pass Task 1 and were excluded. Children listened to the audio recording via E-prime, which randomised the order in which the stories were presented for each participant. Children were informed that they would listen to a story and be asked questions at the end. They were told to listen carefully and do their best. The five questions with dichotomous answers were asked first, followed by the two for explaining the justifications regarding morality and intention. Participants' responses were recorded and transcribed 
verbatim. For the categorisation of responses, two raters (the first author and a colleague blinded to the children's diagnoses and the hypotheses of the study) independently coded all 360 justifications (Cohen's kappa $=0.75$; good strength of agreement). Disagreements between coders were resolved through discussion. Also, to examine whether the age could affect the responses of the MT task, we calculated the main effect of participants age on MT task, which was not significant $(F(1,16)=.93 ; p=.59)$.

\section{Data Analysis}

Data analysis was conducted using the statistical package SPSS (v. 24). Non-parametric statistics were used, as the variables of interest did not follow the normal distribution in the two groups (using the Kolmogorov-Smirnov test for normality). Therefore, the data for dichotomous answers were analysed using non-parametric tests (Mann Whitney-U and Wilcoxon Signed-Rank test) and the justifications were analysed using Chi-squared $(\chi 2)$. The significance level for all the analysis was $p=.05$. However, as the comparison in all the analysis was based on the average across the three scenarios, in the first question (differences between ASD and NT group in forced-choice answers) a threshold of significance of $.05 / 3=.017$ was adopted.

\section{Results}

Results based on the dichotomous choice answers of both groups (ASD-NT): Are there any differences between autistic and NT children in forced-choice answers?

In these analyses a threshold of significance was adopted of a significance of $.05 / 3=.017$. The ASD group scored lower (trend towards statistical significance) than the NT children on (1) detection of the transgression $(U=375.00, p=.02, r=.30$, two-tailed) and (2) identification 
of the perpetrator $(U=320.00, p=.02, r=.31$, two-tailed $)$. There were no significant differences between groups on (3) emotions triggered in the victim $(U=372.00, p=.06, r=$ .24 , two-tailed) and (4) perpetrator morality $(U=352.50, p=.13, r=.20$, two-tailed) (see Table 4-1). Means on (5) wrongness of the act were the maximum score in both groups. Following the scenario-analysis detailed above, no significant results were found when forcedchoice answers were compared between groups.

Table 4-1

Mann-Whitney $U$ scores by question and groups in the moral task ( $\min =0$; $\max =3)$.

\begin{tabular}{ccccccccccc} 
& \multicolumn{2}{c}{ Means } & \multicolumn{2}{c}{ Median } & \multicolumn{2}{c}{ Mean Range } & Z & U & $p$ & $r$ \\
\hline & ASD & NT & ASD & NT & ASD & NT & & & & \\
\hline$(1)$. & $2.70(.75)$ & $3.00(.00)$ & 3 & 3 & 28.00 & 33.00 & -2.31 & 375.00 & .02 & .30 \\
$(2)$. & $2.33(.96)$ & $2.83(.38)$ & 3 & 3 & 26.17 & 34.83 & -2.40 & 320.00 & .02 & .31 \\
$(3)$ & $2.67(.66)$ & $2.93(.25)$ & 3 & 3 & 27.90 & 33.10 & -1.86 & 372.00 & .06 & .24 \\
$(4)$ & $1.83(1.08)$ & $2.20(1.09)$ & 2 & 3 & 27.25 & 33.75 & -1.53 & 352.50 & .13 & .20
\end{tabular}

ASD = Autism Spectrum Disorder; NT = Neurotypical children; (1) detection of the transgression; (2) identification of the perpetrator; (3) emotions triggered to the victim; (4) perpetrator morality

Are there any differences between groups in the resolution of the stories depending on the mental states involved in the transgression (lying/blaming, breaking, stealing)?

On dividing the scores among the three stories in the forced-choice answers, significant differences only existed in the Dog story (lying/blaming) between groups (ASD group mean $=$ 3.43, $\mathrm{Mdn}=4 ; \mathrm{NT}$ group mean $=3.83, \mathrm{Mdn}=4 ; U=315, p=.011, r=.33$, two-tailed).

\section{Do these differences exist in the ASD group?}

In the ASD group, differences were found when the sum of the forced-choice answers was compared among stories, specifically between the Dog story - lying/blaming $(\mathrm{M}=3.43$; Mdn 
$=4)-$ and the Car story - breaking $(\mathrm{M}=3.77 ; \mathrm{Mdn}=4 ; \mathrm{Z}=-2.14, p=.032, r=.39$, twotailed); and also between the Car story - breaking - and the Sandwich story - stealing $(\mathrm{M}=$ 3.46; $\mathrm{Mdn}=4 ; \mathrm{Z}=-2.16, p=.030, r=.39$, two-tailed).

\section{Do these differences exist in the NT group?}

In the NT group, no differences were found among the three stories: all of them were answered in a similar way in the forced-choice answers.

\section{Results based on verbal justifications of both groups (ASD-NT).}

\section{Are there any differences between autistic and NT children in justifications regarding action-morality and intention?}

To analyse the verbal information about the act-morality and the intention, responses were classified into categories. Relevant differences were found. The ASD group scored significantly lower than the NT group on the justifications of action-morality in the following stories: Dog story $\left(\chi^{2}(3)=13.60, p=.004, \varphi=.48\right)$, Car story $(\chi 2(3)=13.54, p=.004, \varphi=$ $.47)$ and Sandwich story $(\chi 2(3)=20.18, p<.001, \varphi=.58)$. There were also significant differences in the same way in the justification of intention, as follows: Dog story $\left(\chi^{2}(3)=\right.$ 9.22, $p=.026, \varphi=.39)$ and Car story $\left(\chi^{2}(3)=12.04, p=.007, \varphi=.45\right)$. There was no significant difference in the Sandwich story $\left(\chi^{2}(3)=6.83, p=.077, \varphi=.34\right)$. The mean value is understood as useful in interpreting participant comprehension in the descriptive analysis and its subsequent comparative analysis (see Table 4-2 for means). 
Table 4-2

Mean and Mode (the level of the category) in ASD and NT group ( $\min =0$; $\max =4)$.

\begin{tabular}{lllll} 
& \multicolumn{3}{c}{ ASD } & \multicolumn{2}{c}{ NT } \\
\cline { 2 - 4 } Act-Moral & Mean & Mode & Mean & Mode
\end{tabular}

\begin{tabular}{lcccc}
\hline Dog & 2.47 & cat. iv - inappropriate & 3.30 & cat. i - mental states \\
Car & 2.33 & cat. ii - description & 3.20 & cat. ii - description \\
Sandwich & 2.57 & cat. ii - description & 3.60 & cat. i - mental states
\end{tabular}

\section{Intention}

\begin{tabular}{lcccc}
\hline Dog & 2.67 & cat. i - mental states & 3.47 & cat. i - mental states \\
Car & 2.53 & cat. ii - description & 3.23 & cat. ii - description \\
Sandwich & 2.90 & cat. ii - description & 3.30 & cat. ii - description
\end{tabular}

Categories

i) mental states

ii) description

$\mathbb{Z}$ iii) literal

iv) inappropriate

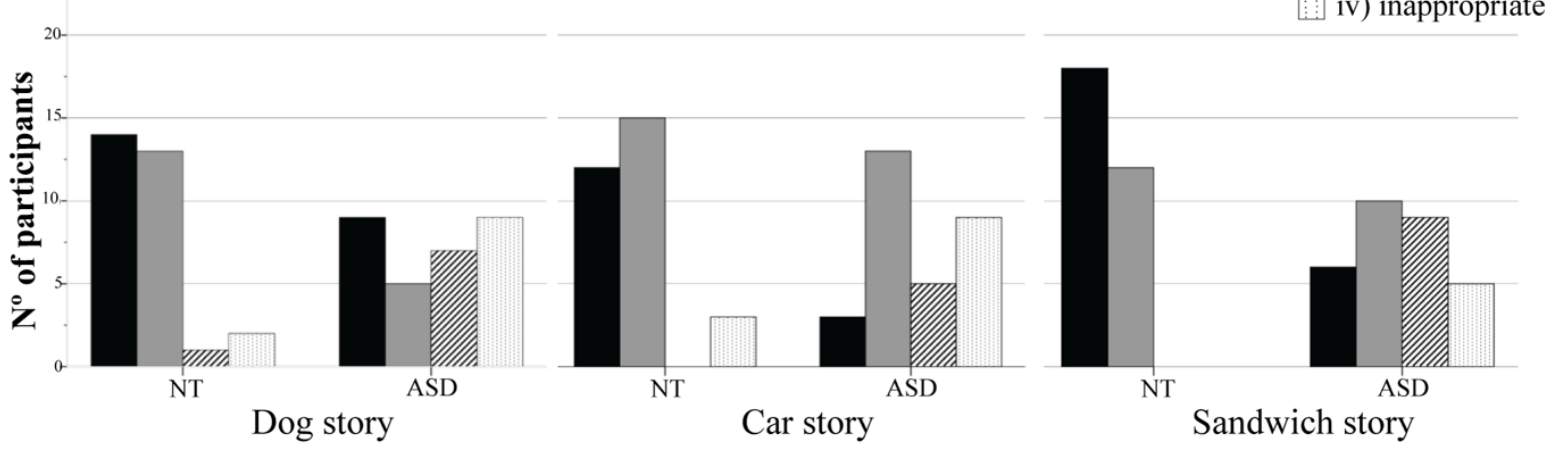

Figure 4-1. Number of justifications put forward by participants with ASD versus NT, classified into categories (i), (ii), (iii) or (iv), in response to action-morality questions on the (a) Dog, (b) Car, and (c) Sandwich story. 


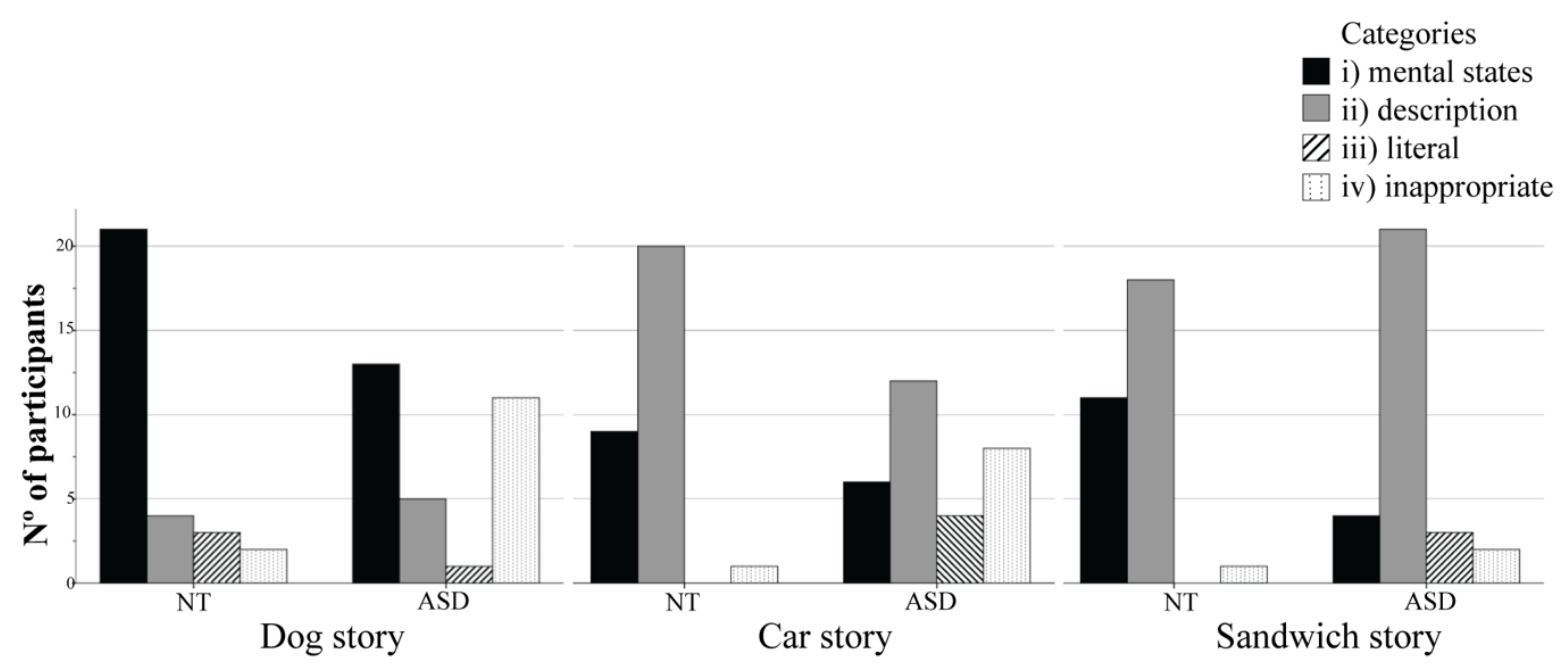

Figure 4-2. Number of justifications put forward by participants with ASD versus NT, classified into categories (i), (ii), (iii) or (iv), in response to intention questions on the (a) Dog, (b) Car, and (c) Sandwich story.

\section{What justifications are more reiterative between groups?}

As shown in Figure 4-1 and 4-2 while the NT children performed well in both the actionmorality and the intention questions, giving responses that fell into categories (i) and (ii) (max scores) across all three stories, the autistic children displayed more varied responses (across all categories), especially regarding action-morality. Thus, the NT group was more focused on the mental states of the characters, and also on the description of the action (e.g., breaking, lying), when it came to reasoning about the action-morality (NT child, real example: "because he ate the food" - classified as category ii: descriptive, in the Sandwich story. See Table 4-3 for more examples). For the intention questions, the NT group focused on the actions, except in the Dog story (Figure 4-2) for which it is important to note that the justifications were based on mental states (NT child, real example: "because if she had told the truth, she would have been punished by her mother" - classified as category (i): mental states. See Table 4-4, category (i) for more examples).

In the ASD group, the justifications were more random in action-morality, with more inappropriate answers and superficial descriptions of the act in comparison to the NT group 
(autistic child, real example: "because he hit it" - classified as category (ii): descriptive). Nevertheless, justifications for intention were commonly better classified in category (ii): the superficial description of the act (autistic child, real example: "because she likes chocolate cakes". See Table 4-4, category (ii) for more examples).

Table 4-3

Morality categorisation and real examples of justifications.

\begin{tabular}{|c|c|c|c|}
\hline Category & Dog story & Car story & Sandwich story \\
\hline i) mental states & $\begin{array}{l}\text { She blamed her dog } \\
\text { without regrets and she } \\
\text { lied her mother. She } \\
\text { made the dog feel very } \\
\text { sad. }\end{array}$ & $\begin{array}{l}\text { He broke the glass of } \\
\text { her friend's car and now } \\
\text { she will be angry and } \\
\text { she will need to pay for } \\
\text { it. }\end{array}$ & $\begin{array}{l}\text { He ate completely the } \\
\text { sandwich of his friend } \\
\text { without permission when } \\
\text { she was not there and } \\
\text { then she was upset. }\end{array}$ \\
\hline ii) description & She lied. & He broke the glass. & Fred stole the food. \\
\hline iii) literal & $\begin{array}{l}\text { Mmm... let me take a } \\
\text { bite of the cake... }\end{array}$ & $\begin{array}{l}\text { "Oliver, what did you } \\
\text { do!?!" }\end{array}$ & $\begin{array}{l}\text { She said: "You've eaten } \\
\text { everything". }\end{array}$ \\
\hline iv) inappropriate & $\begin{array}{l}\text { Dogs used to eat } \\
\text { everything. }\end{array}$ & He likes it. & $\begin{array}{l}\text { It's OK because now she } \\
\text { has more time for } \\
\text { playing. }\end{array}$ \\
\hline
\end{tabular}


Table 4-4

Intention categorisation and real examples of justifications.

\begin{tabular}{|c|c|c|c|}
\hline Category & Dog story & Car story & Sandwich story \\
\hline i) mental states & $\begin{array}{l}\text { She doesn't want that } \\
\text { her mother quarrels her } \\
\text { nor punish her. }\end{array}$ & $\begin{array}{l}\text { He tried to help her } \\
\text { quickly, but the solution } \\
\text { was worst. They should } \\
\text { have called the police } \\
\text { number. }\end{array}$ & $\begin{array}{l}\text { Because he was very very } \\
\text { hungry and he does not } \\
\text { matter anything else. }\end{array}$ \\
\hline ii) description & She loves this cake. & She wanted the keys. & $\begin{array}{l}\text { He loved her sandwich } \\
\text { and she went for water. }\end{array}$ \\
\hline iii) literal & $\begin{array}{l}\text { "She did something } \\
\text { that she should not } \\
\text { have done" (as the first } \\
\text { question). }\end{array}$ & $\begin{array}{l}\text { She said "what could I } \\
\text { do?" }\end{array}$ & $\begin{array}{l}\text { He said: "This sandwich } \\
\text { looks so good!". }\end{array}$ \\
\hline iv) inappropriate & She did. & For hearing the alarm. & He didn't realise. \\
\hline
\end{tabular}

Are there any differences between autistic and NT children in their justifications based on ToM and no-ToM content?

Justifications were re-classified into two categories depending on whether they used mental state terms in their explanations: ToM content and no-ToM content categories. Significant differences between groups were found in the verbal responses of action-morality in the Car story $(\chi 2(1)=7.20, p=.007, \phi=.34)$, and Sandwich story $(\chi 2(1)=10.00, p=.002, \phi=.40)$. In the responses of intention, significant differences were found in the Dog story $(\chi 2(1)=4.34$, $p=.037, \phi=.27)$, and Sandwich story $(\chi 2(1)=4.36, p=.037, \phi=.27)$.

As shown in Figure 4-3 and Figure 4-4, in general, the ASD group gave more justifications classified as No-ToM content compared to the NT group across the three stories. In most action-morality responses to the Car and Sandwich stories, autistic children did not use ToM terms. Significant differences are easily observed in the high number of autistic participants 
who responded with No-ToM terms in Car and Sandwich stories (Figure 4-3). However, in intention responses, both groups were more comparable to each other, with the ASD group always using less mental state terms in their justifications. As in the four categories scores detailed in the previous question, in the Dog story (intention, Figure 4-4), NT group used significantly more ToM-content in their reasoning than in any story, and a high number of participants in both groups gave justifications classified as No-ToM content in the Car and Sandwich stories.
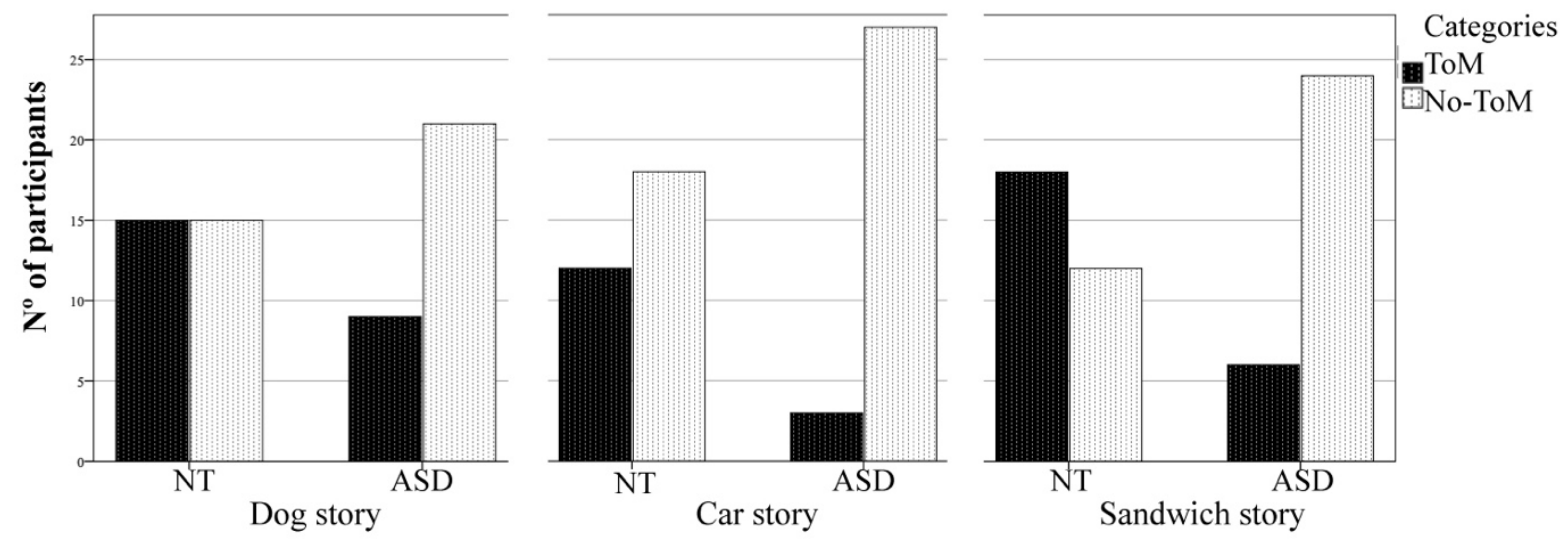

Figure 4-3. Number of justifications put forward by participants with ASD versus NT, classified into categories (i) ToM content and (ii) No-ToM content in response to action-morality questions on (a) Dog, (b) Car, and (c) Sandwich story.
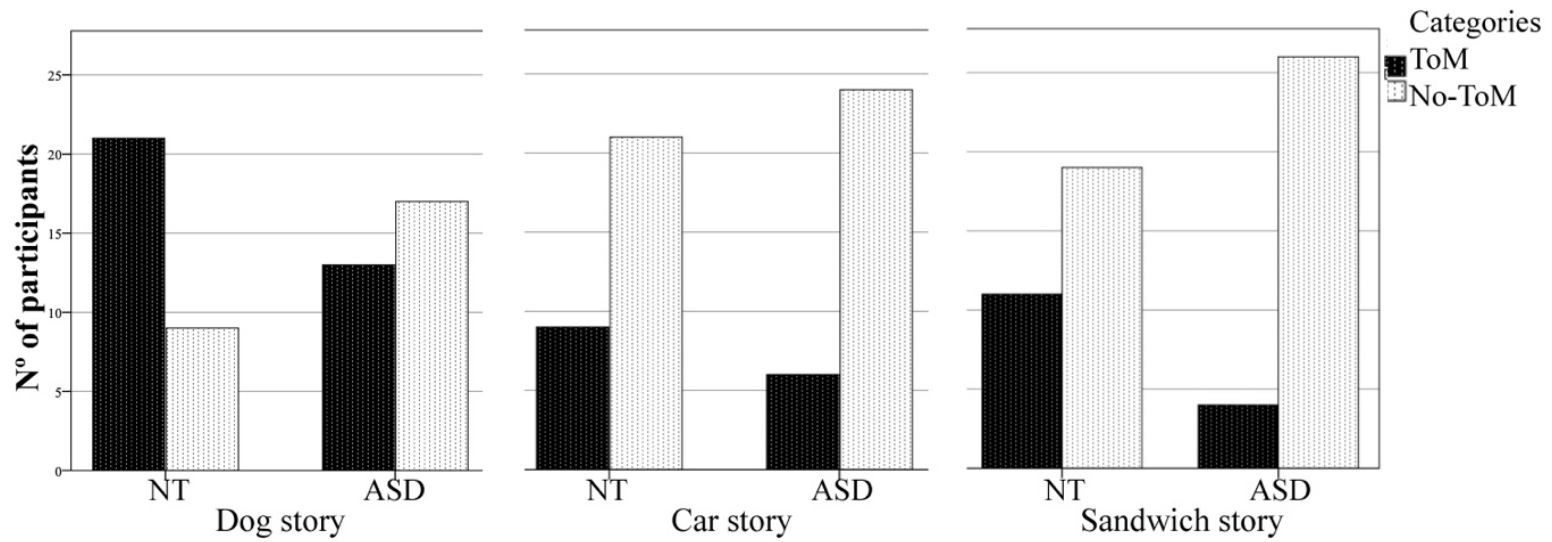

Figure 4-4. Number of justifications put forward by participants with ASD versus NT, classified into categories (i) ToM content and (ii) No-ToM content in response to intention questions on (a) Dog, (b) Car, and (c) Sandwich story. 


\section{Discussion}

The main aim of this study was to investigate whether there were any differences between autistic and NT children on a verbal MT task in forced-choice answers and verbal reasoning.

The findings of this study confirmed our expectations, indicating that moral reasoning was more difficult for children on the autism spectrum, compared to NT participants, especially when mental states are involved in the transgressions. Nevertheless, in our study, autistic children were capable of responding as well as NT children in the forced-choice answers, since the transgressions were unambiguous (bad intention / bad outcome), as Margoni and Surian (2016) argued. Results are discussed in the following.

Firstly, as in Blair's pioneering study, members of the ASD group were sensitive to question (5) about wrongness and were capable of distinguishing the moral transgression correctly. As expected, the capacity to understand wrongness is intact in autistic children when they are asked to distinguish between right/wrong actions. However, the aim of this study goes further in the moral field, in order to gather information about what children on the autism spectrum do and do not understand when other aspects are presented.

As expected, no significant differences were found in relation to the dichotomous choice answers between autistic and NT children. These results are consistent with our first hypothesis based on Leslie et al. (2006), who found basic moral judgements were substantially intact in autistic children. Importantly, in the choice answers, the autistic children showed some understanding of the aspects considered to be 'more difficult', namely, the recognition of basic emotions and the perpetrator-morality. Our results showed that autistic children could distinguish when an action may feel "bad" or "sad" to others. These findings highlighted the importance of others' feelings and emotions and agree with research by Grant et al. (2005), who demonstrated that autistic and NT children (and mild learning difficulty) were able to 
complete their memory and comprehension questions satisfactorily, most of which were related to emotions (i.e., "Did this make John happy or sad?"), as are the questions in the present study. Although in our stories the actions of the characters were always bad, this may be controversial in (3) emotions triggered in the victim in the Car story. For example, some autistic children commented in their verbal justifications, "he did it for her, maybe she could be happy because she retrieved her keys", without considering the bad consequences in their explanations, nor the conflicting emotions caused to the girl (i.e., "however, she is sad or upset because the window is broken"). This is another reason to focus further research on verbal explanations. According to Leslie et al. (2006), the distinction between 'good' and 'bad' is necessary to be able to make basic moral judgements. This fundamental ability underlies the capacity to judge the appropriateness of someone's social behaviour. However, studies usually focus on how bad or good the acts are, and questions related to character-morality are usually asked as culpability judgements or explanations (such as Grant et al., 2005: 'Why do you think X was naughtier?'; see Killen et al., 2011). Our study considered both perpetrator's morality and wrongness (actmorality) in the forced-choice answer questions. As regards the judgement of the character as good or bad, no significant differences between groups were observed because both groups scored the lowest mean value (compared to the other variables) and both were equally unable to correctly judge the perpetrator's morality, as in some of the stories they responded that the character who had caused the bad action was good. This finding is inconsistent with the results in wrongness (as explained in the Method section and Results), since all the participants in both groups judged the action as bad. This discrepancy may be explained by the fact that judging a character can be more difficult than judging an action. Following the model of Cushman (2015), to distinguish intention it is critical to evaluate the morality or culpability of an agent, and the evaluation of the wrongness of the act depends upon the agent's mental states but also on the agent's action. In this study, particularly, the intention and the outcome were not 
ambiguous and children could find it easier to understand the bad action because they linked it to the bad consequences or the victim's emotion, as commented in Margoni and Surian (2016), but they probably do not link it to the agent's mental states. These results highlight one of the reasons why more research is required in the moral field in autism, particularly, on the difficulties in comprehending ecological situations and judging the intentions and morality of others and their acts. Emerging evidence in the criminal justice field shows that autistic adolescents (even children and adults) are more vulnerable to becoming involved in "mate crime" - where someone deliberately befriends an autistic person in order to take advantage of them - as a consequence of the fact that they have these difficulties in interpreting others' intentions and judging whether an act or person is good or bad (Grundy, 2011; Thomas, 2011). These difficulties became especially visible in the resolution of one of the three stories, in which the selfish action resulted in harm to another (the dog). This action affected the dog directly and involved more mental states to understand the reason for that action. The Dog story, as expected, was the most difficult story for autistic children, given that the main act lying - is of such a nature that a certain level of ToM is required for its complete understanding. Furthermore, this story was also considered the most difficult of the three in the second part of the study, when justifications were asked for in the open-ended questions (Talwar and Lee, 2008).

In relation to verbal justification in moral judgements, autistic children showed wider variation, as expected, compared to the NT group. Autistic children had greater difficulties in recognising and explaining intentionality and action-morality in basic moral stories due to the ToM ability required to understand and judge the intention and the morality of the act described in the task. Significant differences were found between autistic and NT children in five of the six explanations. As in previously published research studies (Grant et al., 2005; Shulman et al., 2012), autistic children were able to provide some appropriate justifications, although most 
of them were of poorer quality than those of the NT group, using descriptions of the narrative, reiterations and not attributing mental-state words, as described in Bishop and Norbury (2002). In our study, judgements put forward by the ASD group differed importantly from those of the NT group when the explanations were related to the morality of the act - more so than in the case of intention. This important result is inconsistent with the findings in Blair (1996) and Leslie et al. (2006) based on bad/good/OK choice answers, meaning that ToM could be influencing moral understanding and, especially, the explanations when mental states are involved. Particularly, in their justifications for the Dog story a greater frequency of lower scores were classified - category (iv) - due to more inappropriate answers and verbal reiterations of the story. Therefore, the NT group was a more homogeneous group, with answers predominantly classified between categories (i) and (ii), and the ASD group was more heterogeneous, giving justifications classified in all categories; especially, justifications were classified in the lower categories in the explanations about act-morality (as in Grant et al., 2005). Only considering the ToM (or no-ToM) content in the justifications, graphs provided visual information on how autistic children used less mental state terms in their verbal responses than the NT group in all the stories in both action-morality and intention questions.

In relation to the stories, taking a closer look at their contents of the stories could be useful. For example, the Sandwich story was set in a school, and the scenario was believed to be closer for children than, for example, the Car story, which is more related to adolescents or adults. However, beyond the scenarios and the value of the objects (i.e., car and sandwich), the trust and emotions involved in the stories were more salient for children in the more relatable scenario. It is important to highlight that most children appreciated the trust and friendship in the Sandwich story more, as they explained some similar experiences in their justifications. However, in the Car story, both groups simply explained what was happening and they did not look at the feelings or the culpability of the character arising from breaking an expensive object. 
Possibly, the most striking difference between groups was in the Dog story, which was the easiest to explain for the NT group by attributing mental states to the child's intention. On the other hand, the ASD group tended, on average, to provide repeated elements of the story, which were descriptive, rather than using their own words.

\section{Limitations}

There are some limitations of the present study that should be recognised. First, a limited number of stories were used and group sizes were moderate. Therefore, future studies could include a greater range and variety of moral scenarios such as telling white lies, deceit, cheating, or hitting; and also test larger samples. In addition, we should recognise that the stories were novel and the scenarios were created by the research team. Thus, for future research they could be compared to another advanced ToM tasks, such as Strange Stories (Happé, 1994a) or the Faux Pas test (Baron-Cohen et al., 1999). With regard to the scenarios presented, there is a need for more novel ecological stories and tasks related to real life within the context of children's and adolescent's interaction are needed. One finding that may account for this is based on the differences in content of the scenarios presented. For example, the Sandwich story was set in a school. We noticed that children performed best on tasks that were closest to their own experiences and with dialogues that they could experience in their daily life. In Grant et al.'s (2005) study, the good scores in the ASD group were explained by the fact that they learned by their own experience or because they were explicitly taught. The lowest scores could be explained by the effect of working memory and this must be controlled for in future research, although of course working memory is required in peer-to-peer dialogues and other real-world social situations. Indeed, working memory would be relevant to handle and interpret the information, given all the elements of verbal content one needs to remember and comprehend. For this reason, some working memory control measures should be analysed 
in relation to moral judgements. In a study by Amit and Greene (2012), working memory tasks were used to identify the cognitive styles of NT individuals. As predicted, individuals with visual cognitive styles made more deontological judgements than individuals with verbal cognitive styles; i.e., disapproving of killing someone to save several others. Furthermore, in Moore, Clark and Kane (2008), NT persons with a higher working memory capacity found certain types of 'killing' more appropriate than did those with lower working memory and were more consistent in their judgements. In addition, future studies should further explore the differences between autistic children and comparison participants using moral situations with visual and verbal formats, considering differences in working memory - especially in the verbal information - and, as commented earlier, different scenarios by focusing on the comprehension of emotions, morality, intentions and beliefs.

Overall, difficulties associated with ToM, along with intrinsic understanding of morality and possible links to poor judgement, provide a rationale for further research on the different aspects of the interrelatedness of ToM and moral reasoning, as is evident from recent studies (Killen et al., 2011; Shulman et al., 2012). Another explanation for the difficulties found in the justifications lies in the broad area of complex information processing (Minshew and Goldstein, 1998), the weak central coherence theory (Frith, 1989) and executive function (Ozonoff, 1997; Russell, 1997), in the sense of ignoring distracting information, planning, processing, memorising and working with complex information to decode the story and formulate appropriate answers, based on their own beliefs. It can be summarised that ToM is a multidimensional process that requires the integration of multiple components to understand and predict both our own and others' mental states. 


\subsection{Estudio 2 / Study 2}

La comprensión de las meteduras de pata en escolares con autismo. Su relación con variables cognitivas y de teoría de la mente.

Garcia-Molina, I. \& Clemente-Estevan, R.A. (under review). La comprensión de las meteduras de pata en escolares con autismo. Su relación con variables cognitivas y de teoría de la mente. Universitas Psychologica. 


\section{Resumen}

El presente estudio tiene una doble finalidad. La primera es analizar dos de las variables implicadas en la comprensión de las meteduras de pata en el autismo (Cociente Intelectual; CI, y la Falsa Creencia; FC). La segunda, examinar en detalle las dificultades encontradas en la tarea de metedura de pata, dividida la población con autismo en dos grupos según su CI. De dicha tarea de metedura de pata, se adaptaron cuatro historias (viñetas y narración) de BaronCohen, O'Riordan, Jones, Stone, y Plaisted (1999), y se administraron a dos grupos de niños y pre-adolescente con autismo $(\mathrm{N}=34)$ de entre 7 y 12 años, que las resolvieron a partir de respuestas de elección dicotómica, y de explicación verbal. Los resultados demuestran la capacidad predictiva del CI verbal y de la FC; como también diferencias significativas tanto en algunas respuestas de elección como en su explicación. Estos resultados se discuten profundizando en la comprensión que tienen los escolares con autismo sobre comportamientos sociales, su relación con el CI y la FC, y su posible repercusión en su vida diaria.

Palabras clave: metedura de pata, teoría de la mente, autismo, falsa creencia, inteligencia.

\section{Lay Abstract}

The present study has a double purpose. The first is to analyse two of the variables involved in the understanding of the faux pas in autism (Intelligence Quotient - IQ - and False Belief FB). The second is to examine in detail the difficulties encountered in the Faux Pas test, when autistic individuals were divided into two groups according to their IQ. Thus, two groups of children with autism $(\mathrm{N}=34)$ between 7 and 12 years of age took part of this study. There were eighteen children were in the Group 1, all of whom had a typical or high average IQ. Group 2 contained sixteen children, who all had a low average IQ. Four stories from the Faux Pas test (Baron-Cohen, O'Riordan, Jones, Stone, \& Plaisted, 1999) were adapted (vignettes and narration in Spanish) (see 7.3. Appendix 3). The faux pas stories were based on daily life 
situations in which the speaker said something awkward or behaved in an inappropriate way that hurt the listener emotionally. The person who commits the faux pas, however, would have a false belief of the reality and would not realise that the statement or behaviour is inappropriate (he/she acted accidentally). To resolve the test, the participants responded to closed (choosing among different forced-choice answers; e.g., Yes/No) and open questions (verbal explanations). The results demonstrated the predictive capacity of full-scale IQ $(r=.53 ; p<$ $\left..001 ; \mathrm{R}^{2}=.28\right)$ and FB $\left(\mathrm{r}=.51 ; p<.001 ; \mathrm{R}^{2}=.26\right)$ in the performance of the Faux Pas test. Additionally, a multiple regression analysis explained that verbal IQ and FB could explain $40 \%$ of the variability in the results of the Faux Pas test. The model excluded the performance IQ (Blocks subtest). In addition, when the autistic individuals were divided into the two groups defined above, significant differences were found overall in the forced-choice responses, and more specifically in ii) the recognition of the faux pas speaker, and vii) the ignorance of the information, as well as in their verbal explanations. Group 1 always scored better than Group 2, and thus it could be concluded that the higher the cognitive level is, the better the score in the task will be. The difficulties in the recognition of the faux pas speaker by Group 2 (low average IQ) would be related to the speaker's belief and what he/she says. That is, the lower level group would not actually understand the awkward statement, but instead would judge the listener's comments inappropriately and deliberately. Findings are discussed in depth due to their possible repercussion on the daily lives of autistic individuals.

Keywords: faux pas, theory of mind, autism, false belief, intelligence. 
El auge poblacional y el especial interés en el ámbito clínico, evolutivo y educativo que plantea el autismo, ha repercutido en la definición de detalles más precisos sobre los criterios utilizados en su diagnóstico, así como en la clasificación de sus dificultades que a partir del nuevo DSM-5 (APA, 2013) definen dicho trastorno. Como es conocido, entre las dificultades más comunes en el autismo se encuentran los problemas en la comunicación e interacción social, como, por ejemplo: su disposición reducida por compartir intereses o emociones, los problemas en iniciar o responder a interacciones sociales, las limitaciones en interpretar estados mentales durante dichas interacciones, o las dificultades para ajustar su comportamiento con el fin de encajar en diferentes contextos sociales, entre otros. Estas limitaciones, que ya per se son identificativas del Trastorno del Espectro Autista (TEA), pueden verse aún más afectadas dependiendo del nivel intelectual de la persona con autismo, llegando a repercutir dicha dificultad no solo en el ámbito académico, sino también en sus relaciones sociales y afectivas. En la actualidad, es común que niños y niñas con autismo se encuentren escolarizados en centros ordinarios, especialmente aquellos que por su nivel cognitivo y conductual pueden seguir las exigencias de los curricula escolares y de las normas de relación e interacción de los grupos de iguales. Este marco inclusivo que hoy en día rige nuestros centros educativos, ofrece oportunidades de relación e interacción social a los escolares con autismo. Siguiendo la importancia de la comprensión de estas situaciones sociales, en el presente trabajo se pretende profundizar en un aspecto característico del autismo como es el déficit en la comprensión de los estados mentales de los otros y su relación con la inteligencia general. Diferentes estudios, han relacionado la inteligencia con varios ámbitos de la vida de las personas con autismo, como el éxito escolar y académico (Eaves y Ho, 1997; Mayes y Calhoun, 2003), los comportamientos adaptativos o la independencia personal (Charman, Pickles, Simonoff, et al., 2011; Duncan y Bishop, 2011). Más afín con la propuesta de este estudio, se encuentra el clásico trabajo de Happé (1994b). Esta autora, relacionó los problemas en tareas mentalistas con las dificultades 
en la prueba de Comprensión (de la escala Wechsler) en adolescentes con autismo. La relación se estableció de manera significativa para esta prueba verbal, pero no para el desempeño de la prueba manipulativa Cubos; constatando así, la importancia del efecto de las tareas lingüísticas para detectar posibles problemas ligados a creencias, intenciones o deseos interpersonales. Por tanto, el nivel verbal cognitivo sería un factor importante a analizar, ya que podría afectar dependiendo de su grado - a la comunicación y a la construcción de nuestras relaciones interpersonales, sobre todo en su comprensión e interpretación de situaciones sociales, lo que se conoce como entender la mente de los demás, o en tener Teoría de la Mente (Durrleman y Franck, 2015; Fisher, Happé, y Dunn, 2005; Milligan, Astington, \& Dack, 2007).

La Teoría de la Mente (TM; término acuñado por Premack y Woodruff en 1978) es la habilidad que nos permite predecir, entender y explicar el comportamiento de los otros basándonos en lo que pensamos o sabemos acerca de los conocimientos, creencias y deseos ajenos (Frith y Frith, 2005). Su desarrollo, como todo proceso madurativo, se establece según diferentes hitos o niveles que se alcanzan progresivamente con la edad y la complejidad de las relaciones sociales (Astington, 2001; Wellman y Lagattuta, 2000; Wellman y Liu, 2004). Por esta razón, se han desarrollado tareas sencillas para niños pequeños, las conocidas como de primer orden (de contenido inesperado o de cambio de contenido), y tareas más complejas de segundo orden para escolares, que incluyen mayor grado de comprensión para la toma de perspectiva (ponerse en el lugar de un personaje de la historia de forma recursiva con coste de la memoria de trabajo). Estas tareas se han administrado a niños preescolares y escolares con y sin autismo (Baron-Cohen, Leslie y Frith, 1985; Wimmer y Perner, 1983), principalmente con la finalidad de evaluar aspectos cognitivos fríos (creencias), dando relativamente poco valor a los aspectos de TM más cercanos al ámbito socio-afectivo, de naturaleza más personal y ecológica. Sin embargo, hasta donde se conoce a día de hoy, existen pocos estudios y tareas de diagnóstico o intervención de este último tipo, más relacionados con situaciones cercanas a 
la realidad y a la interacción diaria de niños y niñas próximos a la pubertad. Estas tareas, además de sus implicaciones emocionales, morales y de intencionalidad, suelen contener un considerable soporte lingüístico (gramatical y de uso) con significado ligado a la intencionalidad pragmática del hablante. Por toda esta amalgama de requerimientos y apreciaciones mentalistas, tareas como la utilizada en este trabajo: la de metedura de pata son consideradas de TM de alto nivel, por el majeo de estrategias más complejas que se van perfilando según el desarrollo evolutivo, y que cuya ausencia puede tener consecuencias desadaptativas en la vida del escolar.

\section{Meteduras de pata}

Una metedura de pata (faux pas en francés; término más utilizado en los estudios internacionales) se produce cuando existe un error social embarazoso o un desliz en la conducta, en los modales de interacción, o en las reglas sociales. Según Baron-Cohen, O’Riordan, Jones, Stone, y Plaisted en su trabajo de 1999, para ilustrar de una forma más cotidiana la definición de faux pas, se debería incluir el "Oh-oh" de reacción, el ";Ojalá no hubiera dicho eso!", o el gesto de taparse la boca en actitud de arrepentimiento por la conducta verbal producida. Un ejemplo de historia que incluye una metedura de pata sería la siguiente: Inés ayudó a su mamá a hacer un pastel de manzana para su tío, que vino a visitarlas. Ella lo llevó hasta su tío y le dijo: «Lo hice solo para ti». «Mmm», se le ve buena pinta... —respondió el tío Tomás, «Me encantan los pasteles, el único que no me gusta nada, nada, jes el de manzana!» (historia original de Baron-Cohen et al., 1999; ver 7.3 Apéndice 3).

Como es conocido, las personas con autismo suelen cometer meteduras de pata en sus interacciones, o suelen perderse en las pistas o los enunciados, mostrándose ingenuos o teniendo dificultades en entender y justificar correctamente algunos comportamientos que puedan implicar la comprensión de estados mentales del nivel de estas tareas o historias (como 
bromas, ironías, engaños, mentiras) ya sea en la vida diaria o en la comprensión de pruebas (Sotillo y Rivière, 2001). Por esta razón, es de suma importancia profundizar en los procesos cognitivos mediadores que se incluyen en estas tareas más complejas. En este sentido, para la comprensión de la metedura de pata sería necesario, por un lado, detectar que el emisor no sabe o no recuerda cierto hecho (actúa no intencionadamente) y, por otro lado, apreciar el impacto emocional (habitualmente negativo) que puede hacer el comentario sobre el oyente. Por tanto, existe una diferencia entre el nivel compartido de conocimiento sobre el tema que tienen el emisor de la metedura de pata y el oyente (Baron-Cohen et al., 1999). Es decir, recordar la información presentada, reconocer los estados mentales - intenciones y deseos - de los protagonistas intervinientes y las emociones provocadas. Además, sería importante remarcar la atención a pequeños matices por parte del espectador, detalles concretos que son clave para entender el daño emocional al personaje-oyente. En el caso del ejemplo, la metedura de pata se produjo por el detalle concreto de que el pastel que no le gustaba era únicamente el de manzana - siendo el pastel de Inés de manzana; así pues, el matiz "manzana” sería significativo también para la total comprensión de la historia.

Por esta complejidad, en uno de los trabajos de referencia obligada, Baron-Cohen et al. (1999), se hipotetizó su posible dificultad para niños de entre 7 y 11 años con y sin autismo. Los niños con TEA - con una media igual o mayor en las pruebas de inteligencia manipulativas y verbales, y habiendo resuelto correctamente tareas de primer y segundo orden de Falsa Creencia (FC) - tuvieron más dificultad en responder correctamente a las preguntas sobre meteduras de pata que los del grupo control. En este estudio, se corroboró que, aunque los niños con TEA pudieron identificar los estados mentales de los personajes en las tareas de primer y segundo orden administradas al inicio del experimento, fallaron en entender tareas mentalistas más sociales y ecológicas como las meteduras de pata y discernir, de este modo, su posible impacto en el otro. Curiosamente, se hallaron problemas en la resolución de esta misma 
prueba, en pacientes con Síndrome de Tourette (Eddy, Mitchell, Beck, Cavanna y Rickards, 2010), TDAH-Combinado (Maoz, Tsviban, Gvirts, Shamay-Tsoory, Levkovitz, Waterberg y Bloch, 2014) y esquizofrenia (Guinea, Tirapu-Ustárroz, Pollán, 2007).

Uno de los primeros estudios de réplica en francés de la tarea de metedura de pata, se centró en las respuestas de adultos con autismo con un CI normal (Zalla et al., 2009). El trabajo reveló que los individuos con autismo, aunque detectaron la metedura de pata e identificaron a la persona que la cometió, fallaron en las preguntas sobre la falta de información del personaje que provocaba la metedura de pata y sobre el impacto emocional resultado del comportamiento inadecuado en el personaje afectado. Además, en general, fueron incapaces de proporcionar justificaciones correctas en términos de razones o intenciones en la descripción de lo ocurrido. Por ejemplo, raramente mencionaron las emociones provocadas a los personajes afectados; por otro lado, referente a las explicaciones del faux pas, describieron sesgadamente las intenciones como maliciosas, con propósito de humillar u ofender al otro, o incluso mediante términos no apropiados para describir al personaje (egoísta, arrogante, estúpido). Las respuestas de explicación aportarían pues, más información sobre las dificultades encontradas por las personas con autismo en este tipo de historias más complejas. Sin embargo, pocos estudios han contemplado puntuar o categorizar este tipo de explicaciones en análisis más cualitativos.

El objetivo principal de este trabajo consiste en averiguar la relación de la comprensión de la tarea comentada de metedura de pata, con la FC y el nivel intelectual (representado por el CI) en el desempeño demostrado por un grupo de niños y niñas con autismo; así como conocer la influencia del CI dividiendo el grupo de participantes en dos grupos en función de su nivel. La tarea se analizará a través de respuestas de elección (siguiendo el estudio de Banerjee, Watling y Caputti, 2011), pero también a través de razonamientos de explicación (como en Zalla et al., 2009). De este modo, el presente estudio, nace con la finalidad de avanzar 
en la comprensión que tienen los escolares con autismo sobre los comportamientos sociales, siendo extrapolables las respuestas en la tarea de metedura de pata a la posible comprensión de situaciones reales (Banerjee y Watling, 2005).

Presentados los objetivos, en primer lugar, y considerando el grupo completo de escolares con TEA, se pretende:

1. Averiguar si la FC y el Cociente Intelectual Total (CIT) son predictores de la tarea de metedura de pata. Por lo tanto, se espera que las variables analizadas: CI (total, prueba lingüística y prueba manipulativa del WISC-IV; Wechsler, 2005) y tarea de FC, sean predictores de la resolución de la tarea de metedura de pata. Además, se espera que las variables de FC y el Vocabulario (prueba lingüística) sean mejor predictores de la tarea de metedura de pata que la prueba más manipulativa Cubos (siguiendo la propuesta de Happé, 1994b).

En segundo lugar, dividida la muestra total en Grupo 1 (mejor nivel de puntuaciones de CI) y Grupo 2 (nivel más bajo de puntuaciones de CI), se pretende:

2. (a) Comparar si los niños diagnosticados de TEA clasificados en el Grupo 1 responden y entienden mejor la tarea de metedura de pata que los del Grupo 2 a través de las respuestas a las elecciones dicotómicas. $\mathrm{Y}$ en este sentido, se espera, que haya diferencias en tres de las variables analizadas como en el estudio de Zalla et al. (2009): emociones provocadas, intención de la acción e ignorancia.

2. (b) Examinar si el Grupo 1 responde mejor a la única cuestión en la que se pedía argumentar las razones de las meteduras de pata, y determinar si existen diferencias entre grupos. Así como analizar qué tipo de errores cometen en las explicaciones los dos grupos. 


\section{Metodología}

\section{Participantes}

La muestra del estudio estuvo formada por 34 participantes diagnosticados de TEA, de los cuales 30 eran niños y 4 niñas, todos ellos escolarizados en centros ordinarios. El rango de edad estaba comprendido entre los 7 (88 meses) y los 12 años (152 meses), con una media de edad de 115 meses.

Todos los participantes fueron diagnosticados de TEA por uno o varios psicólogos especializados, siendo administradas pruebas específicas para su diagnóstico diferencial: ADOS (Lord, Rutter, DiLavore y Risi, 2008) y ADI-R (Lord, Rutter y Le Couteur, 1994). Además, los resultados de la Escala de Inteligencia de Wechsler para niños (WISC-III; Wechsler, 1991), nos permitieron la clasificación diferenciada de los dos grupos. La diferencia entre grupos fue de 1 desviación típica entre ellos en el CI Total.

Para la hipótesis 2 ( a y b) el Grupo 1 se compuso por 16 niños y 2 niñas $(\mathrm{N}=18 ; \mathrm{M}=$ 112.17 meses; DT $=19.60)$, con un rango de CIT entre 100 y $120(\mathrm{M}=109.56 ; \mathrm{DT}=7.73) . \mathrm{El}$ Grupo 2 se compuso por 14 niños y 2 niñas $(\mathrm{N}=16 ; \mathrm{M}=118.06$ meses; $\mathrm{DT}=17.42)$, con un rango de $\mathrm{CI}$ entre $70-85)(\mathrm{M}=81.10 ; \mathrm{DT}=5.85)$. Por lo referente a la edad (meses), no difirieron significativamente $(\mathrm{t}=1.09 ; \mathrm{p}<.30)$; en cambio, tal y como se esperaba, las diferencias en el CI fueron muy significativas $(t=-11.83 ; \mathrm{p}<.00)$.

\section{Medidas}

Además de las puntuaciones totales de la escala WISC-III, para determinar los CI, se usaron específicamente las puntuaciones de la prueba de Cubos (prueba manipulativa) y Vocabulario (prueba verbal) de la escala WISC-IV (Wechsler, 2005). 
Falsa creencia $(\boldsymbol{F C})$. Se administró la tarea de FC no-verbal de cambio de contenido usada en Schick et al. (2007) adaptada a su vez de De Villiers y De Villiers (2012). La tarea trataba de una secuencia de imágenes, donde un primer personaje $\mathrm{X}$ encontró un objeto inusual (un pollito) el cual guardó en una caja que originalmente contenía unos zapatos (cuyo dibujo constaba en la caja). Observada la historia, en la viñeta final, se le preguntaba al escolar acerca de la creencia del segundo personaje Y (no presente cuando ocurrió el cambio de contenido). Las preguntas de evaluación fueron: ¿Qué iría aqui?, ¿Qué cree Y que hay dentro de la caja? y ¿Qué hay realmente dentro de la caja? Las opciones visuales presentadas (de reconocimiento) fueron un pollito y unos zapatos. La puntuación máxima a obtener fue de 3 puntos en la tarea (1 punto por acierto). Ver 7.6 Apéndice 6.

Metedura de pata. Se administraron cuatro de las 10 historias originales del estudio de Baron-Cohen (1999). Éstas fueron: 1) historia de la cocinera, 2) historia del avión, 3) historia de los baños y 4) historia del pastel de manzana. Las historias fueron reescritas en castellano por el equipo e ilustradas (con el consentimiento necesario) por la primera firmante de este estudio, en Garcia-Molina et al. (2016). Ver 7.3 Apéndice 3. La presentación se realizó mediante el programa multimedia E-prime para ordenador con pantalla táctil. Cada historia consistió en dos viñetas y un audio recreando las diferentes situaciones de metedura de pata. Para controlar la dificultad en la memoria de trabajo, las imágenes quedaron permanentes mientras se realizaban las preguntas y respuestas. Los personajes que aparecían en las historias eran tanto adultos como niños de género masculino y femenino.

Cuestiones de Reconocimiento. Al finalizar cada historia, una serie de preguntas fueron realizadas y el niño debía responder a las preguntas de forma dicotómica de elección ( $\mathrm{Si} / \mathrm{No})$ pulsando en la pantalla la respuesta correcta. Cada pregunta tenía su respuesta dibujada; por ejemplo, en el caso del Si o No, había dos botones dibujados con un $\mathrm{Si}$ y un No; en el caso de 
la pregunta sobre personajes, estaban las caras de los personajes dibujados de la historia. Toda la información sobre las preguntas dicotómicas fue captada por la pantalla táctil del ordenador.

Las preguntas de reconocimiento y con soporte visual permanente, fueron las siguientes (la respuesta correcta se identifica en cursiva). Todas ellas fueron administradas, aunque la respuesta fuese errónea. Las seis preguntas fueron presentadas en este mismo orden fijo. La forma de puntuar las preguntas dicotómicas fue: $0=$ respuesta errónea; $1=$ respuesta correcta.

i) Detección del enunciado equivoco en la historia: ¿Alguien dijo algo que no debería haber dicho? [Si/No].

ii) Reconocimiento del personaje: En ese caso, ¿quién? [El personaje que comete la $M P(\mathrm{~A}) /$ El personaje que se siente ofendido (B)/Nadie]

iii) Emoción provocada: ¿Cómo se habrá sentido B (el personaje ofendido)? [Bien/Contento; Mal/Triste].

iv) Intención del personaje: ¿Tú crees que A (el personaje emisor) quería hacerle sentir así? [Si/No].

v) Moralidad del acto: ¿Tú crees que lo que hizo A está bien o está mal? [Bien/Mal],

vi) Afirmación de la comprensión no-literal: ¿Crees que alguien metió la pata? (Sí/No).

vii) Ignorancia: ¿Crees que (A) sabía de la relación entre B y la trasgresión? (Sí/No).

Finalmente, se planteó una última pregunta abierta para su explicación con la cuestión: (¿Por qué alguien dijo algo que no debería haber dicho?), la cual se grabó y guardó en el programa como audio para su posterior calificación. Esta pregunta fue contemplada para observar las diferencias entre grupos en la hipótesis 2(b), siendo excluida para la verificación de las hipótesis 2(a), ya que la influencia lingüística de las explicaciones podría repercutir en las correlaciones y predicciones en el CI (sobre todo verbal) y FC. 
La forma de puntuar las explicaciones fue la siguiente (se especifican ejemplos reales siguiendo la historia de la tarta de manzana):

$0=$ No sabe o no contesta; responde algo absurdo. Ejemplo: "porque no era su cumpleaños". 1= Recuerda lo que se dijo. Ejemplo: "porque él dice: el único que no me gusta nada, nada... es el de manzana”.

$2=$ Recuerda lo que se dijo y el personaje afectado (emoción implicada). Ejemplo: "porque hizo que Inés se pusiera triste porque se lo dijo en la cara, que no le gustaba el de manzana".

3= Recuerda lo que se dijo, el personaje afectado (emoción implicada) y la intención accidental del emisor (desconocimiento/el no recuerdo/el accidente del emisor). Ejemplo: "porque él no sabía que era de manzana, y dijo que no le gustaban delante de ella, y a ella le sentó mal, se puso triste, porque lo había preparado con amor".

La autora principal de este trabajo y una colega independiente de dicha investigación puntuaron las respuestas según el criterio establecido, siendo su índice de fiabilidad del 90\% (muy fiable). Las dudas se resolvieron tras su discusión.

\section{Procedimiento}

Anteriormente a la aplicación del material, se pidieron los permisos pertinentes a las autoridades autonómicas y escolares. Una vez conseguidos, se realizó una reunión con el equipo directivo, psicólogos y maestros de los centros. Tras la aceptación de los centros, la familia o tutor legal del niño/niña firmó los permisos correspondientes para su valoración. Como resultado de estas reuniones, 34 niños con un diagnóstico formal de autismo de 5 centros públicos y 1 centro privado de atención neuropsicológica (todos ellos de la Comunidad Valenciana, España) participaron en el trabajo. Todos los participantes tenían las puntuaciones 
del WISC-III actualizadas por un máximo de 1 año, por lo que la división de la muestra se realizó siguiendo el criterio del profesional en los resultados de dicha escala. En los días sucesivos, se administraron las tareas de FC y la de metedura de pata en espacios habilitados para ello: sin distractores, con una mesa y dos sillas. La duración de la sesión se calculó en 25 minutos aproximadamente por participante. Se descartaron 3 participantes por no entrar dentro de los parámetros de CI establecidos y la muestra se clasificó por grupos según su nivel en el CI Total.

\section{Resultados}

Los datos se analizaron a través del paquete estadístico SPSS 24. Las puntuaciones en CI, FC y metedura de pata, se analizaron inicialmente, - hipótesis 1- a través de un análisis de regresión lineal simple y múltiple. Seguidamente, para las hipótesis 2a, por cumplir la muestra las características propias para el análisis paramétrico, las puntuaciones en la tarea de metedura de pata fueron analizadas mediante el test $t$ para muestras independientes. Para la comparación de la hipótesis $2 \mathrm{~b}$, se usaron pruebas no-paramétricas (U de Mann-Whitney). Para todos los análisis, el valor de $p$ para establecer la significación estadística fue de .05.

En primer lugar, para dar respuesta a la primera pregunta: si la FC y el CI son predictores de la tarea de metedura de pata (hipótesis 1), se estimó primero, la regresión lineal simple de la prueba de FC con la tarea de metedura de pata. La hipótesis de que la FC está positivamente relacionado con la resolución de la tarea de metedura de pata fue probada $(r=.51 ; p<.001)$. La relación entre las variables viene explicada por la ecuación: $Y=15.03+3.69 X$. Además, se encontró que la pendiente $\beta=.51, t(33)=3.35, p<.002$; fue estadísticamente significativa y por tanto se aceptó la hipótesis de relación lineal entre FC y la tarea de metedura de pata. El valor de $\mathrm{R}^{2}$ fue de .26 indicando así que el $26 \%$ de la variabilidad de la resolución en la tarea de metedura de pata podría ser explicada por la tarea de FC. 
A continuación, se repitió el mismo análisis de regresión simple con la puntuación total de CI (WISC-III) con la tarea de metedura de pata. La hipótesis de que el CI está positivamente relacionado con la resolución de la tarea de metedura de pata fue probada $(r=.53 ; p<.001)$. La ecuación estimada de dicha regresión viene explicada por: $\mathrm{Y}=5.88+.16 \mathrm{X}$. Se encontró que la pendiente $\beta=.53, t(33)=3.53, p<.001$; fue estadísticamente significativa y por tanto se aceptó la hipótesis de relación lineal entre CI y la tarea de metedura de pata. El valor de $\mathrm{R}^{2}$ fue de .28 indicando así que el $28 \%$ de la variabilidad de la resolución en la tarea de metedura de pata podría ser explicada por el CI total.

Posteriormente, dado el interés en las variables de CI y FC y su relación con la tarea de metedura de pata observada en la regresión lineal simple, se estimó un análisis más exhaustivo mediante la regresión lineal múltiple de las siguientes variables: FC, Vocabulario (prueba lingüística del WISC-IV), Cubos (prueba manipulativa del WISC-IV) con la tarea de metedura de pata.

El análisis de regresión lineal múltiple mostró la existencia de una relación entre las variables que viene explicada por la ecuación: $\mathrm{Y}=11.02+3.11 \mathrm{X} 1+0.55 \mathrm{X} 2$; siendo excluida la variable Cubos en el análisis del modelo. En la Tabla 4-5, se pueden observar los coeficientes tipificados de las variables FC y Vocabulario y sus valores de probabilidad. El valor de $\mathrm{R}^{2}$ en las dos variables predictores: FC y Vocabulario fue de .40 , indicando así el alto grado de variabilidad de la tarea de metedura de pata que se vio explicada por las variables FC y Vocabulario. Así mismo, en ambas regresiones se comprobó mediante el análisis de residuos que los datos se ajustaban bien a los supuestos del modelo de regresión lineal. 
Tabla 4-5

Coeficientes tipificados y valores de probabilidad.

\begin{tabular}{lccc}
\hline & B & SE B & B estandarizado \\
\hline Constante & 11.02 & 2.42 & \\
Falsa creencia & 3.11 & 1.04 & $.43^{* *}$ \\
Vocabulario & .55 & .21 & $.38^{*}$ \\
$* * \mathrm{p}<.005 ;{ }^{*} \mathrm{p}<.05$ & & &
\end{tabular}

Seguidamente, para dar respuesta a la segunda hipótesis 2a: si el Grupo 1 responde mejor a las tareas de meteduras de pata que el Grupo 2, la muestra fue dividida según los criterios explicados en el apartado Participantes, y se observaron las puntuaciones en ambos grupos en las sub-respuestas dicotómicas de elección y el total de la tarea de elección (Ver Tabla 4-6 para medias y desviaciones típicas). El Grupo 1 obtuvo mejor puntuación en la suma total de las respuestas de toma de decisión, siendo la diferencia significativa $(t=-3.69 ; p<.001)$. Las diferencias significativas se encontraron en: ii) personaje $(t=-3.63 ; p<.001)$; y en iv) ignorancia $(t=-3.58 ; p<.001)$. No se encontraron diferencias significativas ni en iii) emoción provocada ni en iv) intención, tal y como se esperaba en la hipótesis 2a.

Tabla 4-6

Medias y DT de las respuestas dicotómicas de elección y Suma Total.

Medias (DT)

\begin{tabular}{lcc}
\cline { 2 - 3 } & Grupo $1($ CI 100-120) & Grupo 2 (CI 70-85) \\
\hline i) Detección & $3.72(.46)$ & $2.94(1.34)$ \\
ii) Personaje & $3.50(.71)$ & $2.50(.89)$ \\
iii) Emoción & $3.50(.62)$ & $3.31(.79)$ \\
iv) Intención & $3.28(.96)$ & $2.56(1.21)$ \\
v) Moralidad & $3.67(.49)$ & $3.25(.93)$ \\
vi) Comprensión & $3.17(1.30)$ & $2.63(1.67)$ \\
vii) Ignorancia & $3.06(.94)$ & $1.75(1.18)$ \\
Suma total tarea & $23.89(3.80)$ & $18.69(4.40)$ \\
\hline
\end{tabular}

Puntuación mínima en la Media: 0; Puntuación máxima en la Media: 4; Puntuación máxima Total: 28. 
En referencia a la hipótesis $2 \mathrm{~b}$ sobre las diferencias entre grupos en la pregunta de explicación: ¿Por qué alguien dijo algo que no debería haber dicho?, se valoraron sus explicaciones repartiendo los puntos según la adecuación de las respuestas $(0,1,2$ o 3; ver el apartado Medidas), siendo 3 puntos el máximo y 0 puntos el mínimo.

Se encontraron diferencias significativas entre los dos grupos $(Z=-2.35 ; p<.019)$, proporcionando el Grupo 1 respuestas más adecuadas que el Grupo 2. Para una observación más gráfica, la Figura 4-5 muestra el número de niños en cada grupo y sus puntuaciones en las diferentes historias según la adecuación de sus explicaciones. En ella, se observa que, algunos niños del Grupo 1 pudieron alcanzar la máxima puntuación: identificando lo que se dijo, haciendo alusión al personaje afectado e indicando la intención por desconocimiento, el no recuerdo o el accidente del emisor. En cambio, ningún niño del Grupo 2 alcanzó la máxima puntuación. También se distingue que, en ambos grupos, una cantidad importante de niños puntuaron 0 (no sabe o no contesta/responden algo absurdo) o 1 (solo identifican lo que se dijo, obviando al personaje afectado y la acción) en las cuatro historias.

Siguiendo con el ejemplo de la historia de la tarta de manzana, se podría decir que se percibieron problemas en ambos grupos en la identificación de: el personaje afectado (porque lo dijo el tío Tomás delante de Inés y/o eso puso triste a Inés) tal y como ya ocurrió en las respuestas dicotómicas relativas al personaje, y de la intención por desconocimiento/accidente del personaje emisor de la metedura de pata (porque él no sabía que la tarta era de manzana). Así pues, en las verbalizaciones, ambos grupos demostraron que mayoritariamente no habían detectado el personaje que cometía la metedura de pata, ni entendido su intención accidental, reincidiendo en sus explicaciones acerca de que Inés hizo el pastel a propósito de manzana, o acusándola de la información que no tenía sobre los gustos de su tío. Ejemplos del Grupo 1: "porque ellas le hicieron una tarta al tío Tomás del sabor que no le gustaba"; o "porque Inés debería saber que no le gusta el pastel de manzana, y al tío le debería gustar”. Ejemplos del 
Grupo 2: "porque le mintieron" o "porque le metió manzana". Obsérvese también el número de palabras utilizadas - explicaciones más largas en el Grupo 1 que el 2 - o los verbos utilizados. Aunque en ambos grupos se denotó la intencionalidad de los actos de la madre y la hija (Inés), en el Grupo 1, los verbos fueron más mentalistas, relacionados con la información de la historia - saber, gustar; en cambio los verbos del Grupo 2, fueron más duros y directos; y el verbo “meter" (relacionado con la comprensión de 'meter' la pata) apareció recurrentemente en las explicaciones del Grupo 2. Sin embargo, se encontró que la mayoría de los niños, tanto de un nivel como del otro, recordaron lo que dijo el personaje (el único que no me gusta es el pastel de manzana). Haciendo hincapié en esta respuesta simple, muchas veces pudo suceder que la respuesta se puntuó con un 1 por repetición exacta de la propia historia ecolalia o repetición literal-, ya que la mayoría de los niños respondían citando literalmente lo ocurrido en la historia o lo que el personaje decía durante el diálogo (el único que no me gusta nada, nada, es el de manzana), y por tanto no entendiendo la historia en sí misma.

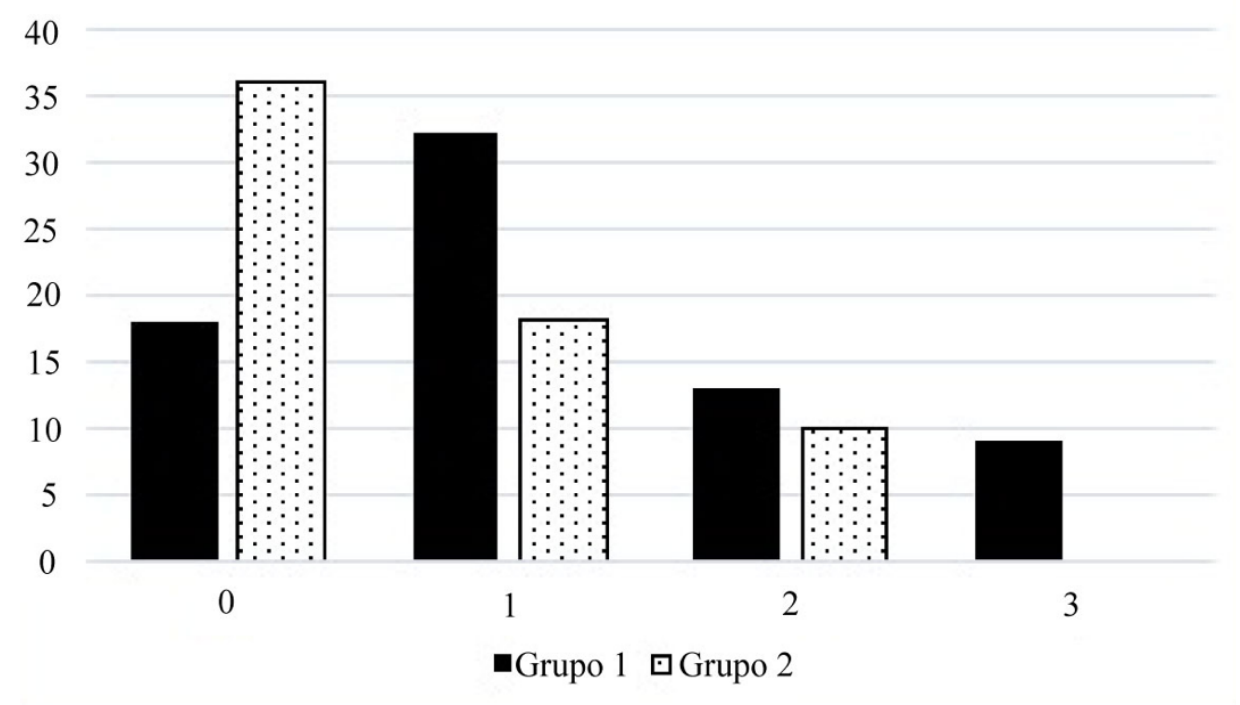

Figura 4-5. Número de niños por grupo y sus puntuaciones en explicación en las historias de metedura de pata. 


\section{Discusión}

El objetivo principal de este estudio fue averiguar en primer lugar, la capacidad predictiva que la FC y la inteligencia - y en particular, en uno de sus representantes verbales más clásicos (Vocabulario) - tenía sobre la tarea de metedura de pata. En la segunda parte del presente estudio, el grupo fue clasificado según su nivel de inteligencia para observar las diferencias entre grupos en dicha tarea de metedura de pata, de forma cuantitativa y cualitativa. Así pues, diversos hallazgos relevantes se han considerado en el presente estudio:

Primero, según las predicciones, se encontró que tanto la variable FC como el CI fueron predictores en la resolución de la tarea de metedura de pata. Después de un análisis más complejo, se halló que la prueba de lenguaje (Vocabulario) estaba influyendo significativamente - junto con la FC - en esta relación. Estos primeros resultados, fueron en la línea del estudio clásico de Happé (1994b) en el que se encontró correlación entre la TM y la prueba verbal de Comprensión. Además, tal y como también se produjo y se esperaba en la hipótesis 1, el modelo de regresión múltiple rechazó la prueba manipulativa (Cubos) de la escala de inteligencia, no siendo esta aceptada para la predicción. Esta predicción de las variables FC y CI, corrobora la importancia de entender la interacción respecto a los aspectos afectivo-sociales y mentalistas de los personajes; y, por otro lado, la comprensión gramatical y pragmática ligada a la intención del hablante (al lenguaje representado por la prueba Vocabulario). Algunos estudios que analizaron tareas de TM cognitivamente más complejas, han argumentado su dificultad por la influencia de la memoria de trabajo verbal en el recuerdo de la historia (Ozonoff, Pennington, Rogers, 1991). Así también, la explicación de esta diferencia entre las dos pruebas de CI, se argumentó por Happé y Frith (2006) a través de atribuir a una teoría diferente de la TM - la Coherencia Central Débil (CCD) - la distinta ejecución en estas capacidades. La CCD sugiere que las personas con TEA necesitarían un 
menor esfuerzo en la habilidad de segmentación de elementos, hecho demostrable por sus altas puntuaciones en la ejecución de la prueba Cubos (Happé y Frith, 2006).

Una vez la población total de niños con TEA fue dividida en dos grupos según su nivel intelectual, se observaron diferencias significativas en la puntuación total de la tarea de metedura de pata, con la mejor resolución por parte del Grupo 1 (grupo con el CI más alto). Así pues, el nivel intelectual estaría influyendo en la resolución de la tarea de metedura de pata, de forma que, a mayor nivel cognitivo, mejor puntuación en la tarea. Aunque la primera explicación podría deberse, tal y como se comentaba en líneas anteriores, al CI verbal, cabría destacar que la tarea, en esta fase, fue totalmente visual, así como sus respuestas de identificación dicotómicas: Sí/No, Bueno/Malo; es decir, el participante tan solo eligió entre opciones y no justificó ni expresó mediante lenguaje aquello que había entendido. Por tanto, el CI verbal podría estar afectando más a la dificultad lingüística y pragmática de la comprensión de los diálogos y de la narración del planteamiento de la tarea; ya que, el peso en memoria de trabajo se trató de controlar mediante la permanencia de las viñetas durante las respuestas.

Tras un análisis más detallado de las cuestiones planteadas, se encontraron solo diferencias significativas entre grupos en ii) personaje e vii) ignorancia. Las dificultades en la ii) detección del personaje - quién mete la pata - por parte del Grupo 2, estarían relacionadas con la creencia de los escolares más en consonancia con la acción real (ella hace la tarta de manzana, por tanto, ella se equivoca), que con la razón mentalista (el tío Tomás dijo algo que no debía por su falta de información) y los estados mentales propios y los atribuibles a los personajes (el escolar que responde tiene conocimiento de la historia y los personajes no). Siguiendo con las diferencias encontradas, sería destacable la pregunta acerca de vii) ignorancia, pudiendo ser clave para la comprensión total de la metedura de pata. Dicha pregunta sobre la ignorancia del personaje (por ejemplo: ¿Acaso el tío Tomás sabía que la tarta era de manzana?) se encontraría altamente ligada a los estados mentales atribuibles a los 
personajes de las diferentes historias; es decir, a la información al alcance del niño-espectador, pero con desconocimiento por parte del personaje emisor de la metedura de pata. En el estudio original de Baron-Cohen et al. (1999) la pregunta sobre ignorancia se formuló para detectar si el escolar entendía la metedura de pata como consecuencia de la creencia falsa del hablante, más que como una acción de intención maliciosa.

Por otro lado, así como en el estudio de Zalla et al. (2009) se detectaron problemas en emociones por parte del grupo con autismo, por lo que se refiere a nuestro estudio, ambos grupos respondieron a las preguntas dicotómicas sobre emociones provocadas correctamente. De hecho, éste fue el punto más fuerte del Grupo 2; aunque, cabría remarcar que, en las cuestiones de reconocimiento, las respuestas eran tan solo dos emociones básicas (Contento/Triste). Este hecho se vio contrastado con resultados diferentes en las preguntas de explicación detalladas a continuación.

En la argumentación de sus razonamientos - pregunta final de explicación general de la tarea de metedura de pata - hipótesis $2 \mathrm{~b}$-, ambos grupos encontraron dificultades, sobre todo en términos de emociones, intencionalidad y desconocimiento de la información. La mayor parte de las puntuaciones se obtuvieron en la barra de 1 punto (ver Figura 4-5), hecho que muchas veces se relacionó con la ecolalia o repetición literal de parte del diálogo, debido a la capacidad memorística del escolar, y no a la interpretación mentalista de la historia narrada. Así pues, existiría una diferencia notoria en el hecho de reconocer mediante elección (preguntas dicotómicas) y de expresar libremente la razón general de la tarea, tal y como se puede observar en el ejemplo de las iii) emociones y la iv) intención. Es en esta pregunta general, en la que el profesional se puede percatar de que pocos niños con autismo alcanzan su comprensión completa. Esta diferencia según el tipo de respuesta afecta a la disparidad de resultados en la detección de emociones y el reconocimiento de la intención real (accidental) en las preguntas cerradas y abiertas. Por ejemplo, fueron escasos los participantes que expresaron léxico 
emocional en las verbalizaciones, aunque ambos grupos respondieron adecuadamente en la pregunta dicotómica de emociones. Además, se denotó cierta culpa e intencionalidad en sus explicaciones hacia Inés, hecho que se correspondería con los hallazgos en Zalla et al. (2009) con descripciones maliciosas y términos no apropiados hacia el personaje; observándose además que el Grupo 2 dio más respuestas inapropiadas en este sentido (frases más cortas y ofensivas) que el Grupo 1 (ver resultados en hipótesis 2(b) para más ejemplos).

Así pues, la pregunta final resultaría de suma importancia para entender qué es lo que realmente los escolares con autismo entienden y creen sobre la situación. El hecho de no entender o no saber explicar estos comportamientos sociales como accidentales o de no percatarse del daño emocional que podía sufrir el otro, podría repercutir en la vida diaria de estos niños y en su relación con los otros. Esta declaración, confirmaría los resultados encontrados en Banerjee et al. (2011), los cuales indicaron que el rechazo por parte del grupo de iguales estaba impidiendo la comprensión de las meteduras de pata. Al mismo tiempo, se halló que las dificultades en entender estas meteduras de pata, eran un claro predictor del rechazo por parte de los pares. Con más razón, Banerjee y Watling (2005) concluyeron en su estudio que las respuestas en las historias de metedura de pata eran un índice útil y efectivo para la comprensión de los comportamientos sociales reales. Siendo esto así, son necesarios, por tanto, materiales nuevos que trabajen TM de alto nivel o centrados en aspectos pragmáticos del lenguaje, tanto de diagnóstico como de intervención, y teniendo en cuenta el nivel cognitivo y comprensión mentalista del individuo. Tareas que, tal y como se ha analizado en este estudio, superen el simple hecho de reconocimiento, el uso mecanicista y simple, y de situaciones no contextualizadas; es decir, combinar las intenciones con las emociones en una situación real.

Cada vez más, los niños con autismo se encuentran en ambientes inclusivos en escuelas e institutos, entornos en los cuales la interacción social prima entre sus iguales, y es necesario 
el trabajo en situaciones sociales y de relación que suelen ocurrir regularmente en su día a día, como entender las meteduras de pata, juzgar acerca de las situaciones morales o captar las sugerencias indirectas.

\section{Limitaciones y líneas futuras}

Como limitaciones, se debe reconocer que la muestra de este estudio es pequeña, y aunque, la mayoría de nuestras hipótesis han seguido el sentido que esperábamos, se debe ser cauteloso con la generalización de los resultados. Además, cabe remarcar que, tras la experiencia del material construido y utilizado, cabría la posibilidad de realizar otros trabajos o materiales que también ofrezcan apoyo visual a historias complejas narradas en diferentes situaciones sociales de nivel interactivo y pragmático complejo, como juicios morales o ironías. Además, con el fin de entender de forma más detallada las dificultades de comprensión en estas tareas de alto grado mentalista, se podría incidir en más preguntas dirigidas a la comprensión de las situaciones y de los estados mentales implicados. Por ejemplo, con más de una pregunta relacionada con las emociones (tanto de la persona afectada como del emisor de la metedura de pata), o con la moralidad del personaje y de su pretensión intencional (el personaje es bueno porque no lo hizo a propósito; aunque lo que hizo estuvo mal). En definitiva, materiales didácticos y de intervención, que puedan incidir en los sesgos mentales - normalmente ingenuos - con respecto a la moralidad, las emociones causadas, las intenciones y las creencias de las personas, dada la necesidad de comprensión que exige nuestra sociedad hoy en día para nuestra adaptación social. 


\subsection{Study 3}

\section{Autism and Faux Pas. Influences of Presentation Modality and Working}

Memory

Garcia-Molina, I. \& Clemente-Estevan, R.A. (in press). Autism and Faux Pas. Influences of Presentation Modality and Working Memory. Spanish Journal of Psychology.

(C) Universidad Complutense de Madrid and Colegio Oficial de Psicólogos de Madrid 


\begin{abstract}
People diagnosed with an autism spectrum disorder (ASD) often have difficulties on Theory of Mind (ToM) tasks involving social situations, such as faux pas. The objective of this study was to find the modality of presentation (visual, verbal, or mixed) that yields the best understanding of a faux pas, and the possible influence of other variables, including intelligence (IQ), age, and working memory. Thirty autistic children and 30 neurotypical children, all aged 7 to 12 years old and comparable in age and IQ, participated in this study. They were asked to resolve nine faux pas stories (three per modality). Significant betweengroups differences were found in the visual and verbal modalities, such that the NT group had higher scores than the ASD group in the Faux Pas test. The ASD group's comprehension was better via the mixed modality than the verbal modality. In addition, working memory had a bigger impact on Faux Pas understanding in cases of autism than in typical development $\left(R^{2}\right.$ explained between .19 and .28 of variance in Faux Pas test outcomes in autistic children), and could therefore explain some of the difficulties previously reported in this area. Future research should include a measure of control for working memory among the stimuli presented to test for group differences in faux pas understanding.
\end{abstract}

Keywords: faux pas; autism; modality; working memory; theory of mind. 
Understanding other people's mental states - and one's own - is central to understanding social behavior, enabling one to interact with and relate to other people. The ability that allows us to attribute, predict, and comprehend mental states is known as Theory of Mind (ToM; Baron-Cohen, Leslie, \& Frith, 1985). At first glance, its definition is simple, but it involves a series of intrinsic and predictive aspects that were previously referred to, almost mystically, as "mind reading." To understand other people's mental states means not only analyzing their intentions, plans, personality, knowledge, emotions, beliefs, and desires; it also requires an understanding of the social context and situation in which events occur (Killen et al., 2011).

Much of our current understanding of ToM is based on studies of autism because autistic people show deficits in their mentalistic understanding of others. For that reason, prototypical ToM tasks have been widely administered to autistic individuals, for instance the classic "Sally-Anne" first-order false belief (FB) test pertaining to location change (BaronCohen et al., 1985). As noted by Killen et al. (2011), this task involves limited social content, because there is no relationship between the characters (for example, if they are friends or strangers), or between the person and object. Nor does the task recognize the intention or motivation that leads the character to take action, or the emotions the action provokes. Changeof-location tasks such as Sally-Anne are referred to as cold, or cognitive ToM, and involve understanding thoughts and beliefs. According to the model proposed by Shamay-Tsoory, Harari, Aharon-Peretz, and Levkovitz (2010), cognitive ToM is a prerequisite to understanding situations that more closely resemble real-life social contexts where empathy and comprehension of emotional states and feelings are the focus - that is, hot or affective ToM. Fewer tests of advanced ToM contemplate 'hot ToM', including the well-known "Strange Stories" (Happé, 1994a), and Faux Pas test (Baron-Cohen, O’Riordan, Stone, Jones, \& Plaisted, 1999), which is the main focus of the current study. 


\section{Faux Pas, Theory of Mind, and Autism}

A faux pas can be defined as making a statement or taking an action that unintentionally turns out to be wrong, inopportune, or socially inappropriate.

Therefore, understanding a faux pas means activating two types of mental state: understanding that the person said/did something they should not have - cognitive ToM - and that the listener is insulted and hurt - affective ToM (Baron-Cohen, Jolliffe, Mortimore, \& Robertson, 1997; Shamay-Tsoory et al., 2007). In other words, to fully understand a faux pas would require someone to, first, detect that the speaker either does not know or does not remember something (unintentional action), and second, to appreciate the emotional impact usually negative - the comment or action may have on the listener (Baron-Cohen et al., 1999). Importantly, attention to specific details can be quite taxing on working memory (WM); for that reason, WM has been controlled for in previous studies of faux pas understanding (see Gregory et al., 2002; Zalla et al., 2009).

\footnotetext{
"The term working memory refers to a brain system that provides temporary storage and manipulation of the information necessary for such complex cognitive tasks as language comprehension, learning, and reasoning” (Baddeley, 1992, p. 556).
}

Given the aforementioned important cognitive and emotional processes that mediate faux pas, autistic people often misunderstand social mores or misinterpret information, making them vulnerable to this type of situation in real life. Those difficulties are also apparent on advanced ToM reasoning tasks (Sotillo \& Rivière, 2001).

Baron-Cohen et al. (1999) created one of the most widely utilized tests of faux pas recognition. Several studies have administered it to autistic people and a neurotypical (NT) comparison group, especially in adults. Their findings indicate that judging different scenarios as they are presented is complex, especially when they involve accidental actions, due to ToM 
difficulties and respondents' particular way of processing important information (GonzalezGadea et al., 2013; Pedreño, Pousa, Navarro, Pàmias, \& Obiols, 2017; Spek et al., 2010; Zalla et al., 2009). According to Zalla et al. (2009), in which study 15 autistic adults and 15 NT adults participated $\left(\mathrm{M}_{\mathrm{ASD}}=28\right.$ years; $\mathrm{M}_{\mathrm{NT}}=27.8$ years $)$, a significant proportion of autistic adults answered incorrectly that the character acted intentionally, believing that the character had deliberately intended to humiliate or offend the other person.

However, at its inception, the Faux Pas test was created to measure advanced ToM in schoolchildren or pre-adolescents. At 7 to 11 years of age, when comprehension of similar situations would mature, children are able to recognize the recursiveness of the mental states involved in a faux pas, and its relationship to intentions, beliefs, and emotions (Baron-Cohen et al., 1999; Pearson \& Pillow, 2016). In their study, the original authors of the Faux Pas test mentioned that autistic children had difficulty recognizing other people's mental states. They found it hard to integrate all the information into a coherent picture, and comprehend the underlying psychological impact of a faux pas (Baron-Cohen et al., 1999). In the same study, NT children (girls and boys) aged 9 and 11 years detected the faux pas, with no significant difference in the performance levels between NT girls and boys when they were 11 years old. Likewise, Pearson and Pillow's (2016) study of NT children (7, 8, 9 and 11 years old) and NT adults found that after 9 years of age, participants were able to recognize the character/speaker's ignorance ( $\mathrm{s} /$ he does not know / s/he does not remember), a fact which the 7 and 8 years old did not detect.

It is noteworthy that in both studies, the youngest NT children - 7 to 9 years old - were unable to recognize all the aspects involved in a faux pas, an ability which seems more mature from nine years of age onward. 


\section{Working Memory Controls in Theory of Mind Tests}

Shifting our attention to working memory, authors such as Gregory et al. (2002) and Zalla et al. (2009) attempted to control the burden on memory and attention - given how demanding a faux pas story is - by placing the text of the faux pas story in front of the participant.

Similar WM controls have been applied to other ToM tasks (mostly first-order FB tasks). Studies of patients with brain damage corroborate the finding that when ToM tasks increase the burden on $\mathrm{WM}$ or executive function (EF), performance drops significantly (Apperly, Samson, \& Humphreys, 2005). Other studies tried to reduce the likelihood of error by using visual stimuli to support WM (Stone, Baron-Cohen, \& Knight, 1998), or shortened the tests to reduce the demands on EF (Apperly, Samson, Chiavarino, \& Humphreys, 2004).

In light of the above, although Baron-Cohen and colleagues (1999) mentioned that their autistic participants do not have WM issues, it remains the case that comprehending a dialogand narrative-based story can be complicated, given all the elements of verbal content one needs to remember.

\section{Verbal Faux Pas}

In studies by Baron-Cohen et al. (1999) and Zalla et al. (2009), participants listened to different faux pas stories, delivered vocally by the test administrator or on a cassette tape. This sort of verbal/auditory modality has been the most widely utilized, along with mixed (vignettes plus audio), due to the importance of the verbal content to overall comprehension of a faux pas story. The controversy, then, lies in whether or not the linguistic and WM demands of these more complex tasks influence participants' responses. Along those lines, the first part of BaronCohen et al.'s (1999) study found that NT children's mistakes on faux pas stories were not due to lack of comprehension or memory, despite a correlation between verbal mental age (VMA) 
and correctly completing the Faux Pas test. They remarked that the correlation could simply be the result of growing maturity, on both measures: older respondents perform better, so mistakes on the Faux Pas test should not be attributed to deficits in linguistic abilities, per se. Those authors conducted a second study as part of the same research, in which autistic children also participated. The authors reported that the significantly worse results in the ASD group were not due to their verbal profiles. Unlike Study 1, no correlation was found between VMA and Faux Pas test resolution, with both groups exhibiting similar VMA (no significant between-groups differences).

\section{Presentation Modality in Relation to Autism}

Interventions have been developed in line with evidence that autistic individuals are helped more by visual interventions than by other modalities (Dettmer, Simpson, Myles, \& Ganz, 2000; Cohen \& Sloan, 2007; Hayes et al., 2010; Johnston, Nelson, Evans, \& Palazolo, 2003). Similarly, in the research literature, autistic individuals score on par with, or outperform comparison groups on visual tasks related to perception and intelligence assessment. That is true of embedded figures (Almeida, Dickinson, Maybery, Badcock, \& Badcock, 2014), block design (Shah \& Frith, 1993), or visual search (Joseph et al., 2017). It is what Blaser, Eglington, Carter, and Kaldy (2014) called the "ASD advantage" in visual tasks. Few studies have examined this visual advantage on more abstract or ecological reasoning tasks, likely because to attribute mental states and emotions to others, verbal information (intonation, pause, indecision, speech, etc.) can sometimes be even more illuminating than visual information (e.g., facial expressions) (see Wiseman's 1995 study of lies in a NT population). In a study by Kleinman, Marciano, and Ault (2001), autistic adults and NT solved an advanced ToM task using two modalities: visual (expression of the eyes) and verbal (tone of voice). As predicted, 
the group of autistic adults performed significantly worse than the comparison group in both modalities, being unable to attribute mental states in either case.

Two of the studies most closely related to visual faux pas are Pierce, Glad, and Schreibman (1997), and Loveland et al. (2001), who explored schoolchildren and adolescents' moral reasoning during social situations. Pierce et al. (1997) found that understanding social situations in moral terms (as correct or incorrect) was harder when autistic children had to attend to several stimuli; as such, a non-verbal scenario would be easier. Loveland et al. (2001) showed a series of videos depicting appropriate and inappropriate social interactions containing verbal stimuli (speech) and non-verbal stimuli (non-narrative scenes). Following Pierce and team's study, Loveland et al. (2001) ventured that the easiest scenes for autistic children and adults to comprehend would be non-verbal. The ASD group had significantly more difficulties than the comparison group identifying inappropriate behavior when the scenario was verbal. For both groups, judging conversations was harder than judging purely non-verbal interactions.

Therefore, the visual "ASD advantage" should not just occur in studies of intelligence assessment; it would also be expected during ecological tasks (containing real-life scenarios). Conversely, verbal ecological tasks and situations with multiple stimuli to attend to can be more difficult to comprehend for autistic individuals.

\section{A Different Way to Process Information}

Further examining the possible influence of verbal or non-verbal presentation, it is worth asking if the so-called visual "ASD advantage" could be explained by Weak Central Coherence theory (WCC; Frith, 1989). WCC entails that autistic people fail to integrate information into an overall context; instead they focus preferentially on pieces of information in an isolated fashion. WCC is another plausible explanation for the superior performance of autistic individuals compared to NT people on tests involving fragmented elements, such as 
the block design or embedded figures tests, because such tasks favor local processing. This poses a question: Is there an advantage to visual problem solving when the stimulus is nonverbal because there are fewer stimuli to attend to, or due to a particular way of processing visual information (as in the block design test)? In fact, Baron-Cohen et al. (1999) proposed that deficient faux pas comprehension could be explained by how autistic people process information - textually citing WCC theory - such that it is laborious for them to reintegrate all the information and realize the true impact of a faux pas statement on the listener.

In light of the above, ToM is definitely a complex process, and it is affected by many other cognitive processes. To ascertain whether extraneous variables such as presentation modality, WM, IQ and age influence comprehension on advanced ToM tasks, the present study tested the influence of these variables on performance in the Faux Pas task.

In greater detail, the present study hypothesizes that (1) there will be significant differences between the groups of autistic and NT children on the Faux Pas test such that the NT group will score higher regardless of modality (Baron-Cohen et al., 1999); (2) we speculate that the most difficult modality for autistic people will be either verbal, or mixed (verbal plus visual stimuli), and we expect significant differences according to modality in the ASD group such that their Faux Pas test outcomes are best in the purely visual modality (Loveland et al., 2001; Pierce et al., 1997); (3) we predict that while WM demands are to some degree controlled (Apperly et al., 2004; Apperly et al., 2005; Stone et al., 1998), WM will correlate with and explain variance in Faux Pas test outcomes in both groups. Furthermore, even though both groups are of a similar age and IQ, we predict that age will influence Faux Pas test outcomes since the study's participants include young children (Baron-Cohen et al., 1999; Pearson \& Pillow, 2016). 


\section{Method}

\section{Participants}

A priori power analyses using $\mathrm{G}^{*}$ Power indicated that a sample size of 26 participants per group would be adequate, with power set at $d=.80, \alpha=.05$ and a desired level of assurance of .80. Sixty participants in total took part in this study, all of them enrolled in mainstream schools. They ranged in age from 84 to 145 months ( 7 years old - 12 years and 1 month old), with an average age of 113.63 months $(\mathrm{SD}=18.71)-9$ years and 5 months old. Thirty participants (5 girls and 25 boys) were diagnosed with level 1 Autism Spectrum Disorder (ASD) by one or more specialists or psychologists, using specific tests for differential diagnosis: the Autism Diagnostic Observation Schedule (ADOS; Lord, Rutter, DiLavore, \& Risi, 2008) and the Autism Diagnostic Interview-Revised (ADI-R; Rutter, Le Couteur, \& Lord, 2003). Another 30 participants ( 7 girls and 23 boys) comprised the neurotypical (NT) group. We compared the two groups according to chronological age (in months); intelligence (IQ, range: 80 - 130); visual WM; and verbal WM. In addition, they all correctly completed the first-order FB ToM task (see Materials). Table 4-7 presents descriptive data and betweengroups comparisons, which did not indicate significant differences on all measures.

Table 4-7

Participants' Descriptive Data, Using Student's t Statistic

\begin{tabular}{lccccccc}
\hline & ASD (30) & NT (30) & & & & \\
& \multicolumn{1}{c}{$\mathrm{M}(\mathrm{SD})$} & $\mathrm{M}(\mathrm{SD})$ & $\mathrm{t}$ & $p$ & $d^{\text {a }}$ & \multicolumn{1}{c}{$C 1.95^{b}$} \\
\hline Age (months) & $112.87(18.58)$ & $114.40(19.11)$ & .32 & .75 & -.11 & $-8.21-11.28$ \\
Intelligence (IQ) & $102.83(14.23)$ & $107.03(12.02)$ & 1.23 & .22 & -.32 & $-2.61-11.01$ \\
Visual WM & $9.59(3.26)$ & $10.57(1.94)$ & 1.41 & .16 & -.37 & $-4.12-2.37$ \\
Verbal WM & $9.69(3.64)$ & $11.33(2.93)$ & 1.92 & .06 & -.50 & $-.07-3.36$ \\
\hline
\end{tabular}

${ }^{\text {a }} d=$ Effect size calculated using the Cohen's $d$ formula; ${ }^{b} C I .95=$ Confidence interval 


\section{Materials}

All tests were administered in Spanish since all the participants speak Spanish. The tests were of intelligence, working memory (visual and verbal), false belief, and the Faux Pas test (visual, verbal, and mixed modality).

Intelligence (IQ). To measure intelligence, we administered Sattler's (1992) shortform adaptation of the WISC-III, Wechsler Intelligence Scales for Children (Wechsler, 1991). WISC-III scores of the autistic children were redacted from their psychologist or neurology specialist's report (maximum 2-year-old report). Total WISC-III IQ scores in autistic children were highly correlated with the short form version (Sattler, 1992). Therefore, the NT group was administered the short-form version to get a reliable estimate of intelligence quotient in that group. The main goal was to guarantee that the two groups had comparable IQ levels.

WM tasks were divided into visual and verbal modalities:

Visual working memory. We administered the Reverse Memory subscale of the Leiter-R (Farmer, 2013), which measures memory capacity using a series of pictures. Respondents are asked to retain the image long enough to replicate the series by choosing the reverse order in which it was initially presented. For example, if the instructor points with his or her finger at "giraffe, frog, shoe," the respondent should select "shoe, frog, giraffe."

Verbal working memory. We administered the Digit Span subtest of the Wechsler scale (WISC-IV) (Wechsler, 2005), using two variants: "forward" and "backward" digits.

First-order false belief ToM task. We administered an adaptation of the non-verbal FB test. Its unexpected content is based on vignettes by De Villiers and De Villiers (2012), in which there is a relationship between characters (they seem to be brothers, and the "object" is an animal/pet). This supports Killen et al.'s (2011) line of reasoning about limited social content. 
Advanced theory-of-mind Faux Pas test. Nine faux pas stories were presented in random order using the E-prime multimedia computer program for touch-screen. For the purposes of the present study, these stories were analyzed according to presentation modality (see Table 4-8 and Appendices 1, 2 and 3 - 7.1; 7.2; 7.3):

Three visual stories. These were taken from Garcia-Molina et al. (2016), and consisted of two vignettes recounting a situation of embarrassment or error. Questions appeared on a screen (without audio) and respondents answered visually (images choice). See 7.1 Appendix 1.

Three verbal stories. Created for the purposes of this study (see 7.2 Appendix 2), these are based on Baron-Cohen et al.'s (1999) Faux Pas test. They include audio that recreates a faux pas situation through dialog and narration (different, very distinct voices were used). The questions were posed via audio (not written) and respondents answered verbally (the student answered out loud, and the program recorded their answers).

Three mixed stories. These vignettes, taken from Garcia-Molina et al. (2016), are also based on Baron-Cohen et al.'s (1999) Faux Pas test. They include vignettes, written narrative, and audio to simultaneously recreate the full situation of a faux pas. Questions appeared on the screen (with audio) and respondents answered visually (similar to the visual stories test). See 7.3 Appendix 3.

Table 4-8

Story Classification by Modality and Error

\begin{tabular}{llll}
\hline Error due to... & Visual & Verbal & Mixed \\
\hline Confusion & Ball & Hair & Plane \\
Lack of information & Present & Exam & Apple pie \\
$\begin{array}{l}\text { Not paying attention or } \\
\text { remembering }\end{array}$ & WC & Birthday & Cubicles \\
\hline
\end{tabular}


To control for working memory, on the visual- and mixed-modality tests, the pictures remained on the screen while the questions were asked and answered. During the verbal stories test, the written text was not placed in front of the participant, but they were verbally reminded of important details. For instance, while the respondent chose who said something strange, or something he/she should not have said, characters' names were repeated along with their own voice: "If so, was Joan (in the boy's voice), Olga (the girl's voice), or no one (narrator's voice)?"

Recognition questions. At the end of each story, the same series of questions was asked - in the visual, verbal, or mixed modality. To answer, the child was asked to choose among different forced-choice answers.

All responses to forced-choice questions (by touch or voice) were captured by the program E-prime and saved for later analysis.

The questions appear below. For ease of comprehension, the correct answer is underlined, character A is the speaker of the faux pas, and B the listener. Every single question was asked, even if the student answered an item incorrectly. The six questions were presented in the same set order, but the stories appeared in random order. Answers were scored as follows: $0=$ incorrect answer; $1=$ correct answer.

1. Faux pas detection: In the story, did someone say something they should not have said? $(\underline{\text { Yes}} / \mathrm{No})$

2. Identification of the character: If so, who? $(\underline{A} / B / N o b o d y)$

3. Emotion elicited: How must B have felt? (Good/Happy; $\underline{\text { Bad/Sad })}$

4. Character's intention: Do you think A meant to make B feel that way? $(\mathrm{Yes} / \underline{\mathrm{No}})$

5. Character's morality: Is A a good or bad person? ( $\mathrm{Good} / \mathrm{Bad})$

6. Morality of the action: Was what A did right, or wrong? (Right/Wrong) 
7. Awareness or ignorance: Do you think A knew (information A does not have/is ignorant of $)$ ? (Yes/ $\underline{\mathrm{No}})$

\section{Procedure}

After schools consented to participate, the boy/girl's family or legal guardian signed the corresponding consent forms for their child to be evaluated. As a result of these meetings, 32 children with a formal autism diagnosis participated in the study, from five mainstream schools and one specialized center for neuropsychological assessment and treatment in Spain; an additional 32 NT children from the same schools and levels were selected by teachers according to established criteria. In the days that followed, they administered the FB and intelligence tests. Depending on their results (passing the first-order FB test and obtaining an IQ score over 80 ), we proceeded with the tests of working memory (verbal and visual) and the Faux Pas test (all modalities) in spaces specially set up for it: clear of acoustic or visual distractions, with a table and two chairs. Assessment took between 75 and 90 minutes per participant and was split into two sessions. Four participants were excluded, not falling within the established parameters, and one did not attend the last session.

\section{Statistical Analysis}

The data were analyzed using the SPSS 24 statistical package. This sample had the characteristics necessary to conduct parametric analysis. According to Kolmogorov-Smirnov's test, the sample was normally distributed; Student's $t$ test for independent samples was used to observe between-groups differences; and paired $t$ tests were carried out to determine if there were differences between modalities within each group - hypotheses 1 and 2. With hypothesis 3 in mind, we started by calculating correlations between WM, IQ, age (independent variables), and Faux Pas test outcomes (dependent variable). Next, we undertook a step-wise regression 
procedure, adding in variables that were initially correlated. For all analyses, a $p$ value of .05 was considered to establish statistical significance.

\section{Results}

\section{Between-groups Differences (ASD - NT) in Faux Pas Test Outcomes}

With regard to hypothesis 1 , between-groups differences were calculated in the three modalities of faux pas - visual, verbal, and mixed. Significant between-groups differences were found when the story's presentation modality was visual and verbal, however differences were not significant in the mixed modality. See Table 4-9.

Table 4-9

Between-groups Differences on Faux Pas Test According to Modality ( $t$ test for independent samples)

\begin{tabular}{|c|c|c|c|c|c|c|c|c|}
\hline & \multicolumn{2}{|c|}{ ASD } & \multicolumn{2}{|c|}{ NT } & \multirow[b]{2}{*}{$\mathrm{t}$} & \multirow[b]{2}{*}{$p$} & \multirow[b]{2}{*}{$d^{\mathrm{b}}$} & \multirow[b]{2}{*}{ CI. $95^{\mathrm{c}}$} \\
\hline & M (SD) & $\begin{array}{c}\text { Range }^{\mathrm{a}} \\
\text { (Min-Max) }\end{array}$ & $\mathrm{M}(\mathrm{SD})$ & $\begin{array}{c}\text { Range } \\
\text { (Min-Max) }\end{array}$ & & & & \\
\hline Visual & $15.60(3.17)$ & $14(7-21)$ & $17.73(2.27)$ & $8(13-21)$ & 2.99 & .004 & -.77 & $.71-3.56$ \\
\hline Verbal & $15.10(2.98)$ & $12(9-21)$ & $17.03(2.70)$ & $10(11-21)$ & 2.64 & .011 & -.68 & $.47-3.40$ \\
\hline Mixed & $16.13(2.85)$ & $10(11-21)$ & $16.33(3.04)$ & $11(10-21)$ & .26 & .794 & -.07 & $-1.32-1.72$ \\
\hline
\end{tabular}

${ }^{\mathrm{a}}$ Maximum score $21 ;{ }^{\mathrm{b}} d=$ Effect size calculated using the Cohen's $d$ formula; ${ }^{\mathrm{c}} C I .95=$ Confidence interval.

Differences according to modality (visual, verbal or mixed) on Faux Pas test. With respect to hypothesis 2 about the differences between modalities (visual, verbal, and mixed), results in the ASD group showed significant differences between verbal and mixed modalities (with lower scores in the verbal modality). The NT group, on the other hand, displayed significant differences between visual and mixed modalities (scoring lower on mixed). See Table 4-10. 
Table 4-10

Differences between Modalities, by Group (Paired t Test)

\begin{tabular}{lccccccc}
\hline \multicolumn{7}{c}{ ASD } & \multicolumn{5}{c}{ NT } \\
\hline Modality & M (SD) & $\mathrm{t}$ & $p$ & $\mathrm{M}(\mathrm{SD})$ & $\mathrm{t}$ & $p$ \\
\hline Visual - Verbal & $15.60(3.17)$ & & & $17.73(2.27)$ & 1.36 & .184 \\
& $15.10(2.98)$ & .85 & .403 & $17.03(2.70)$ & & \\
& & & & & & & \\
Visual - Mixed & $15.60(3.17)$ & & & $17.73(2.27)$ & 2.45 & .021 \\
& $16.13(2.85)$ & -1.15 & .260 & $16.33(3.04)$ & & \\
& $15.10(2.98)$ & & & & & & \\
Verbal - Mixed & $16.13(2.85)$ & -2.48 & .019 & $17.03(2.70)$ & 1.68 & .103 \\
& & & & & & & \\
\hline
\end{tabular}

Effect of WM and IQ on Faux Pas test. Prior to conducting the predictive analyses relevant to hypothesis 3 , we obtained bivariate correlations between $\mathrm{WM}$, intelligence, age, and Faux Pas test results across three modalities.

In the ASD group, significant correlations were found between: visual WM, and visual and mixed Faux Pas; verbal WM, and verbal, visual, and mixed Faux Pas; and intelligence (IQ), with visual and mixed Faux Pas.

Conversely, in the group of NT children, we found no significant correlation among these variables (WM, IQ, and Faux Pas test). In this group, only one correlation was found, between participants' age and mixed faux pas. 
Table 4-11

Bivariate Correlations between Faux Pas, Visual WM, Verbal WM, and IQ in the ASD and NT Groups

\begin{tabular}{lcccccc}
\hline & \multicolumn{3}{c}{ ASD } & \multicolumn{3}{c}{ NT } \\
\cline { 2 - 7 } & \multicolumn{3}{c}{ Faux pas } & & \multicolumn{3}{c}{ Faux pas } \\
\cline { 2 - 7 } & Visual & Verbal & Mixed & Visual & Verbal & Mixed \\
\hline Visual WM & $.44^{*}$ & .23 & $.43^{*}$ & .18 & .35 & .11 \\
Verbal WM & $.49^{* *}$ & $.46^{*}$ & $.42^{*}$ & .00 & .31 & .21 \\
IQ & $.46^{*}$ & .27 & $.38^{*}$ & .19 & .18 & .26 \\
Age & .11 & .28 & .29 & .33 & .29 & $.43^{*}$ \\
\hline
\end{tabular}

*correlation is significant to the level of .05

** correlation is significant to the level of .01

Next, to determine which variables were independent predictors of Faux Pas test results (visual-, verbal-, and mixed-modality), we conducted multiple regression analyses using a stepwise procedure. However, this was only done in the group of autistic children, given their correlations between WM and IQ (see Table 4-11 for detailed correlations by group). We excluded linear regression data from the NT group, about age in relation to mixed Faux Pas, because the model was not found to be significant $(F(1,28)=2.12 ; p=.156)$.

Following the step-wise procedure cited above, significant regressions were found when the model included just the dependent variable and WM. All models excluded IQ. As TaError! Reference source not found. shows, $19 \%$ to $28 \%$ of variability in Faux Pas test $\mathrm{r}$ esults was explained by WM test outcomes (visual or verbal). See TaError! Reference source not found. for all these analyses. 
Table 4-12

Summary of the Regression Analysis and Step-wise Procedure to Predict Faux Pas Test Results in Different Modalities (Visual, Verbal, and Mixed), in the ASD Group

Variables

$F \quad$ S $B$

$\beta \quad t \quad \mathrm{R}^{2}$

\section{Visual Modality}

Model 1

$\begin{array}{ccccccc}\text { Constant } & 6.41^{*} & 11.58 & 1.71 & - & 6.77^{* * *} & \\ \text { Visual WM } & & .43 & .17 & .44 & 2.53^{*} & .19\end{array}$

Model 2

$\begin{array}{ccccccc}\text { Constant } & 8.71^{*} & 11.50 & 1.51 & - & 7.59^{* * *} & \\ \text { Verbal WM } & & .43 & .15 & .49 & 2.95^{*} & .24\end{array}$

Model 3

\begin{tabular}{|c|c|c|c|c|c|}
\hline Constant & $4.92^{*}$ & 10.56 & 1.75 & - & $6.02^{* * *}$ \\
\hline Visual WM & & .21 & .21 & .22 & 1.05 \\
\hline Verbal WM & & .32 & .18 & .36 & 1.72 \\
\hline
\end{tabular}

\section{Verbal Modality}

Model 1

$\begin{array}{ccccccc}\text { Constant } & 7.10^{*} & 11.47 & 1.47 & - & 7.81^{* * *} & \\ \text { Verbal WM } & & .38 & .14 & .46 & 2.66^{*} & .21\end{array}$

\section{Mixed Modality}

Model 1

$\begin{array}{ccccccc}\text { Constant } & 6.12^{*} & 12.58 & 1.55 & & 8.12^{* * *} & \\ \text { Visual WM } & & .38 & .15 & .43 & 2.47^{*} & .19\end{array}$

Model 2

\begin{tabular}{|c|c|c|c|c|c|c|}
\hline Constant & $3.71^{*}$ & 11.96 & 1.63 & - & $7.31^{* * * *}$ & \\
\hline Visual WM & & .25 & .19 & .28 & 1.31 & \\
\hline Verbal WM & & .19 & .17 & .24 & 1.12 & .22 \\
\hline
\end{tabular}




\section{Discussion}

The present study was devised to answer two questions: via which modality do autistic children best understand faux pas? And do certain variables (age, WM and IQ) influence Faux Pas test outcomes?

To begin with, we should point out that hypothesis 1 was consistent with our results in that autistic participants showed difficulty on mentalistic tasks (Baron-Cohen et al., 1999; Zalla et al., 2009). In our study, autistic participants fared worse than NT participants on the Faux Pas test regardless of modality. Significant differences were observed between groups when test presentation was purely visual or purely verbal. That fact reiterates that faux pas comprehension is challenging for autistic people; it is classified as requiring an advanced level of ToM, and involves cognitive as well as affective ToM sub-processes (Baron-Cohen et al., 1997; Shamay-Tsoory et al., 2007). Nevertheless, the two groups scored very similarly in the mixed modality, where there were no differences between groups.

Shifting our attention to Faux Pas test modalities, we shall discuss several novel findings that may have repercussions to related future research, and possible explanations closely related to information processing in autistic individuals.

\section{The Complexity of Verbal Stimuli for Autistic Individuals}

First of all, according to the so-called ASD advantage on visual tasks (Blaser et al., 2014) - especially on mentalistic tasks (Loveland et al., 2001; Pierce et al., 1997) -hypothesis 2 predicted that within the ASD group, the visual modality would have the best outcomes, and that differences might surface between the two modalities with verbal stimuli: verbal and mixed. According to our data, the visual modality was easiest for children in the NT group, with significant differences compared to the mixed modality. Contrary to expectations (it was expected that autistic children would score higher in visual modality), in the ASD group, there 
were no differences between visual and verbal modalities, nor between visual and mixed. The highest score in the ASD group occurred in the mixed modality, and significant differences were only found compared to the verbal homologue. This difficulty with verbal story comprehension for the ASD group could be explained by the high imaginative and symbolic demands of deducing what is happening in a purely verbal scenario, as in a telephone conversation. An important criterion in autism diagnosis is that imagination appears to be limited (see Rutter, Le Couteur, \& Lord, 2003). Meanwhile, that skill was performed by NT participants without issue in the verbal modality of the Faux Pas test.

Another possible explanation for autistic children scoring lower than the NT group in the verbal modality could be that autistic children found it difficult to integrate and understand all the information provided (Baron-Cohen et al., 1999). Moreover, the linguistic and WM burden of comprehending verbal fragments (presented as dialog) in a Faux Pas test, with no other type of support, may have affected overall comprehension of the verbal stories (Apperly et al., 2004; Apperly et al., 2005). However, importantly, Baron-Cohen et al. (1999) argued that between-groups differences on the Faux Pas test could not be attributed to their respective linguistic ability; rather, the ASD group's difficulty comprehending a faux pas story might be explained by how they process the information and comprehend it cohesively, as a whole. That proposal would be supported by WCC theory in autism (Frith, 1989), that is, recognizing the parts without comprehending the big picture. For instance, in the verbal Faux Pas modality, pieces of audio from a dialog (verbal fragments) must lead the participant to make sense of an overall story. NT participants, however, had scarcely any problem solving the Faux Pas questions in verbal or visual modalities. They were able to recognize all the defining aspects of faux pas, understanding it - based on all the information together - as one entity. Additional theoretical support for these results is the theory of neural complexity, which also postulates that autistic people are good at local processing, whereas global processing is impaired. The 
same theory proposes that auditory processing in autistic people is the result of better perception when auditory stimuli are simple and low-level. However, there is altered perception when the auditory information is more complex (Lin, Shirama, Kato, \& Kashino, 2017). Along those lines, Lin et al.'s (2017) study revealed that autistic people tend to show atypical responses to social auditory stimuli, namely, voices. For example, they show less preference for their mothers' speech, and lower attention when someone calls their name, compared to NT children. Moreover, autistic adults often have difficulty perceiving prosody.

Therefore, another possible explanation for the ASD group's results in the verbal modality - in addition to what Baron-Cohen and team argued in their seminal study - could be reduced attention to, or altered perception of, auditory information. Difficulty recognizing voices and interpreting dialog would make that modality more challenging for autistic individuals.

\section{Working Memory in Predicting Faux Pas Test Outcomes}

One of the most striking results is the correlation and prediction displayed by WM in all modalities of the Faux Pas test, specifically, solely in the group of autistic children, and likewise for IQ. These predictive analysis support hypothesis 3, since visual and verbal WM would influence Faux Pas test outcomes, despite our attempt to reduce the demands on memory - as other studies have suggested the same to reduce the effect of WM (Apperly et al., 2004; Apperly et al., 2005; Stone et al., 1998). Whereas in the NT group, WM did not correlate with Faux Pas results in any modality, in the ASD group, visual and verbal WM explained $19 \%$ to $28 \%$ of variability in Faux Pas test outcomes (verbal, visual, mixed), and IQ was excluded from all the regression models. In terms of age, although previous studies reported improved mentalistic test outcomes in older children - over 9 years old (Baron-Cohen et al., 1999; Pearson \& Pillow, 2016), in our study, age correlated only with mixed-modality Faux Pas results in the NT group. Since the predictive model was not significant, it was not included 
in our detailed analyses. Therefore, our participants' age (7 to 12 years) did not affect their answers on the Faux Pas test. The original age range of the test on which our study is based was children up to seven years old. Therefore, our results were in line with the Baron-Cohen study's preliminary sample.

One last possible explanation for the unique influence of WM on remembering central aspects of faux pas in the ASD group is that autistic individuals process information and abstractly assign meaning differently. This is consistent with WCC theory (Frith, 1989). Its premise is that autistic people do not integrate information as an overall whole - generally but instead attend to and preferentially remember small visual (face) or verbal elements (a sentence) separately - locally (see Happé \& Frith, 2006). Along those lines, autistic participants would rely more heavily on WM than the NT group to remember the complete story and answer the test's comprehension questions. Consequently, comprehending and memorizing contingent information as separate elements (without generalizing to form overall meaning) might be hindering overall memory of the correct understanding of the story. On a similar note, BaronCohen and his team (1999) specifically controlled "parroting," because autistic children tend to resort to echolalia or repeat a particular fragment - usually the last thing they were listening to with no concrete meaning. It would stand to reason that "parroting" might also occur during the mental process of listening to the story and the corresponding questions, mentally repeating a particular fragment, and entering into a loop, impeding the person from completely construing the story's overall meaning.

It is certainly the case, as Baron-Cohen and his team concluded, that our results shed some light on the possibility that how autistic people process information may influence their outcomes in situations requiring mentalistic reasoning. Our study contributes the novel finding that WM and presentation modality influence outcomes on advanced ToM testing. 


\section{Limitations}

Some limitations of the present study should be considered. The first concerns the small sample size of our groups. The second issue was the lack of validated measures of Verbal Faux Pas stories, although all the stories were created following the same structure as Baron-Cohen et al. (1999). In addition, for future research, it would be useful to find a more robust way to control WM, better than the measures used in other studies (placing the text in front of the participant) and ours (reminders while the questions were asked). Likely, attending to different stimuli at the same time (reading and listening), or to additional information (voices, background noise, etc.), could affect the final outcome. The third point being, it would be interesting to administer another presentation modality. For example, stories of faux pas in video format could capture variables closer to real-life situations, such as action, gestures, glances, and body language. Finally, as emotion is clearly important in processing faux pas (e.g., one may appreciate the emotional impact that the comment or action may have on the listener), in future research, the effect of emotion recognition would have to be included as a predictor of faux pas understanding.

\section{Conclusion}

This study provides empirical evidence and practical implications for autistic children and adolescents. On the one hand, the study tries to answer the question: What type of presentation is most beneficial to autistic children in advanced ToM tasks, as in the case of Faux Pas test? Results indicate the mixed format (a mix with verbal and visual information) would be the most suitable to access visual information - more closely related to affective ToM - (faces, emotions, details, etc.) and verbal information - related to cognitive ToM - (tone of voice, prosody, dialog, etc.), which are both necessary subprocesses to completely comprehend a faux pas. On the other hand, the present study emphasizes the creation of new stories and 
presentation modalities, to offer new resources to this field of study and practice. More materials based on advanced ToM are needed for assessment as well as intervention purposes. This study's findings also underscore the importance of WM and the attentional demands of verbal or nonverbal stimuli during tasks based on social situations. Therefore, future intervention tools or activities should consider including reminders to enable the participant to follow the thread of the story.

Our findings could be the first step in creating new intervention materials that include simple visual stimuli rather than purely verbal stimuli to improve comprehension of daily-life situations. 


\subsection{Study 4}

\section{Moral judgements in autistic children. Moral Transgressions and Faux Pas stories}

Garcia-Molina, I., Clemente-Estevan, R.A. \& Andrés-Roqueta, A. (under review). Moral judgements in autistic children. Moral Transgressions and Faux Pas stories. Research in Autism Spectrum Disorders. 


\begin{abstract}
This study establishes the possible relation between intention and morality, and the difficulty this entails for autistic children due to their theory of mind impairments. For this purpose, a set of everyday situations that require the capacity of theory of mind were compared: Moral Transgressions $\left(\mathrm{MT}^{3}\right.$ ) and Faux Pas (FP) tasks, the intention of which varied (MT: deliberate, FP: accidental). Sixty-two participants matched by age and intelligence (30 autistic and 32 neurotypical children - NT) answered forced-choice questions about the situations. The competence of the two groups did not differ significantly in either the MT or the FP tasks, as they displayed a similar ability to make an adequate moral decision when using information about intention, rather than using only the outcome. Nevertheless, in the FP task the agent was judged more severely by the autistic group than the NT group (as "bad"), since the final outcome could have influenced their moral decision. In between-group comparisons, the autistic group showed significantly less competence in the MT, when the action directly affected another person (personal mediator). The complexity of FP in terms of intention and beliefs involved is discussed and the subtle difficulties of autistic children are analysed.
\end{abstract}

Keywords: Theory of Mind; Morality; Intention; Autism.

\footnotetext{
${ }^{3}$ MT and FP acronyms were used in this Study due to Moral Transgression and Faux Pas terms being used repeatedly.
} 
The capacity that allows us to understand and hypothesise about mental states is known as Theory of Mind (ToM), and it is crucial for reasoning, making us capable of judging actions and also the agents involved in a specific situation. For example, if we recognise that a child breaks something by accident, the moral evaluation we can make of that child will be different from that formed if the action was carried out deliberately. Hence, understanding the intentions is a key aspect that allows us to attribute moral responsibility to an agent of an offensive or hurtful act (Baird and Astington, 2004; Cushman, 2015; Young and Saxe, 2009; Zalla and Leboyer, 2011). The shift in reasoning from outcome- to intent-based moral judgement has been a critical aspect of development since Piaget's (1932) seminal study. As young children's judgements are outcome-based (due to their immature ToM), also in this sense, it is well established that ToM is often impaired in autistic individuals ${ }^{4}$, and this deficit can affect their moral evaluations. For this reason, it appears relevant to assess whether autistic children have consequently problems to understand that the agent's morality can be influenced by the intention. In this context, the novelty of the present work is that no other study has been found that analyses the moral reasoning in two types of everyday situations which can be classified as advanced ToM tasks. These tasks are related, as ToM ability is necessary to understand the situations and both have a bad outcome. However, they differ in the intent: deliberate situations (moral transgressions, MT) and accidental situations (faux pas; FP).

\section{Moral judgements construction from the deconstruction of the intentional-action}

The folk concept of 'intentional-action' explains how mental states can cause physical events (Cushman, 2015). The understanding of the elemental features that form an intentionalaction enables us to reconstruct its interrelation to moral judgements (see Figure 4-6). The

\footnotetext{
${ }^{4}$ From here onwards the term autistic will be used, see Kenny et al. (2016). The acronym of Autism Spectrum Disorder - ASD will be employed only when groups are compared.
} 
above-mentioned example of breaking something can illustrate this relationship. Imagine that firstly, the girl's plan (intention) was to kick the ball (action) to score a goal (outcome). However, the lack of control over this action implied that the girl accidentally (intention) kicked the ball with her heel (action) and broke a window (outcome which was not planned). Thus, the judgement as to whether the girl was good or bad will depend more upon her intention than the outcome caused (see Cushman, 2015; and Rogé and Mullet, 2011).

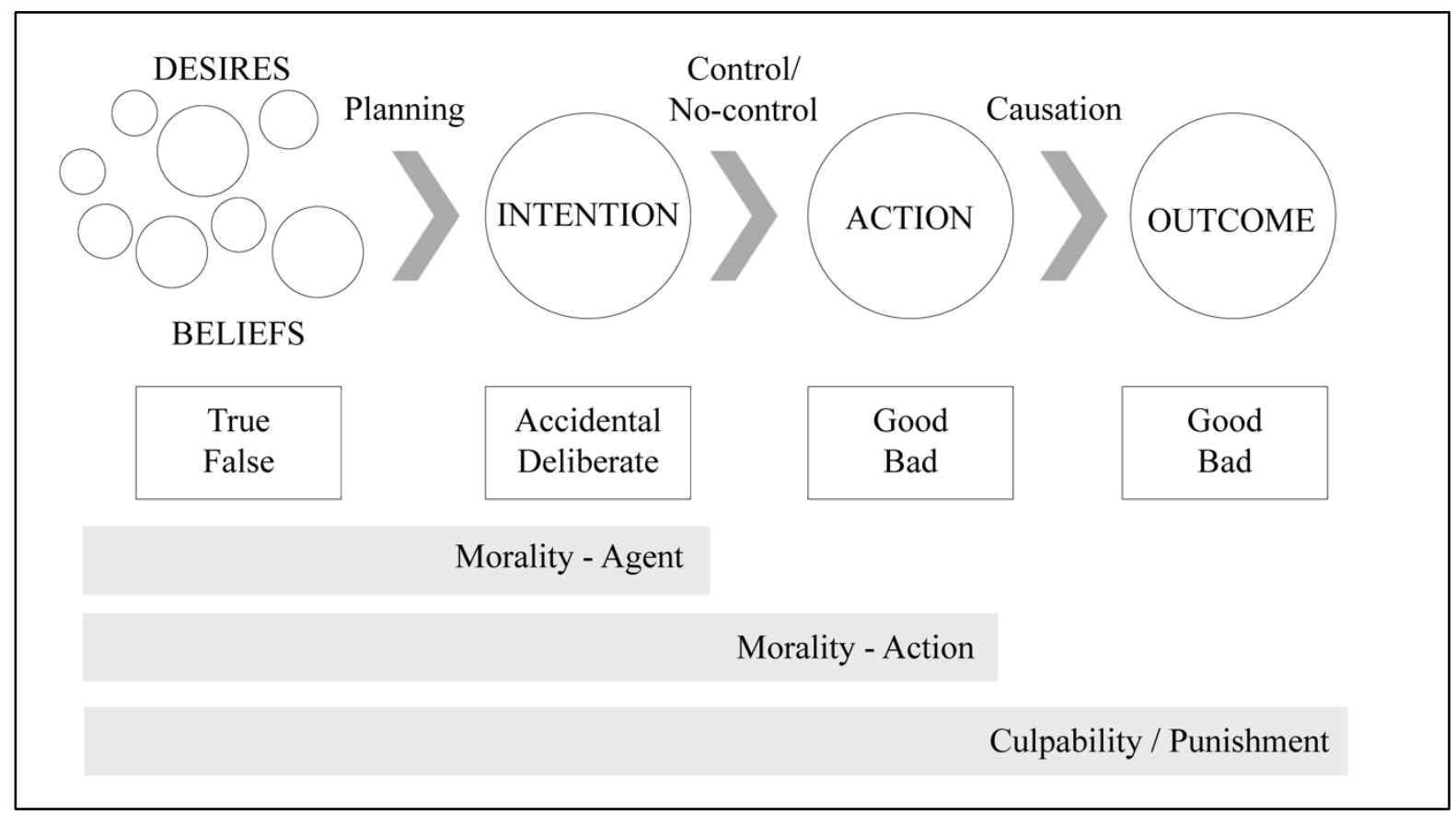

Figure 4-6. Intention of the action broken down into its elemental features and morality (modified from Cushman, 2015).

Figure 4-6 shows the relation between the mental states about others or situations (nonobservable categories) and the actions and consequences (observable categories - events). These actions can be the product of a deliberate intention (plan under control) or an accidental intention (non-controlled plan due to causes such as a mistake, forgetfulness or lack of information). In short, people's knowledge of others - or lack thereof - can give rise to beliefs that may or may not match reality. This case would be different from that explained before (the breaking a window example), because when there is insufficient information, the false beliefs 
affect the deliberate intention of the action, accidentally resulting in a completely different action. Consequently, the final outcome will be neither the one that was expected nor the one planned. For this reason, this new approach of analysing morality in faux pas situations will be explored in the present study.

\section{Accidental action and the Faux Pas test}

The most common task used to explain the accidental situations is the Faux Pas test, and it has been widely administered mainly to autistic people, due to their impairments and the importance of recognising the agents' mental states to understand the socially inappropriate behaviour of the FP stories. As far as we know, the moral implication of the agent who commits the FP (i.e., says an awkward comment) has not been analysed or compared with tasks involving moral scenarios. Recently researchers are paying attention to the FP test, suggesting that even when autistic individuals attribute mental states correctly, intention is not fully used for moral reasoning (Buon et al., 2013; Zalla and Leboyer, 2011; Zalla et al., 2011; Zalla et al., 2009).

Baron-Cohen and his team presented the FP test as a psychologically relevant method by highlighting its mentalist aspects (Baron-Cohen et al., 1999). The task is composed of a set of everyday stories. In each story, the speaker usually has a false belief about reality (he/she does not know some important information), and consequently grasping his/her intention is a key aspect to understand the story. The observer (the participant being assessed) can access all the information and may be able to distinguish the intention of the speaker as accidental (not planned), regardless of whether the action and outcome are judged as 'bad'. In the next example, the two paths cited (speaker's and observer's perspective) can be analysed: "Thea wants to make a negative comment about Will; Thea does not know that Will is nearby and can hear what she says to Anne about him". 
Path 1 (speaker's perspective): Thea has the desire to say to Anne that Will looks weird and both of them believe that they are alone and nobody can hear them (False belief) - Thea plans the comment (Deliberate - $1^{\text {st }}$ intention) - Thea says the comment as she planned (control over the action) - Thea communicates her comment to Anne (Neutral Outcome).

Path 2 (observer's perspective): Thea has the desire to say to Anne that Will looks weird, but Will is close to them and can hear everything (True belief: Will is hiding and listening to them)

- Thea does not plan to hurt Will (Accidental $-2^{\text {nd }}$ intention) - Thea makes the offensive comment without controlling for certain aspects (No control over the action) - Thea's comment hurts Will (Bad Outcome).

Therefore, the recognition of a faux pas depends specifically on the understanding of false beliefs and intentions, and these are aspects that autistic people often misinterpret. In this sense, Zalla et al. (2009) observed that autistic adults could correctly detect the faux pas statement (someone said something that he/she should not have said), but they failed to interpret the false belief of the speaker. Moreover, they provided explanations in terms of malevolence by judging the speaker's intention to humiliate or offend the listener as deliberate.

\section{Intention- or outcome-based judgements.}

When there is an emotional impact (e.g., someone being hurt), autistic people tend to make judgements of the agent based on the consideration of the outcome, instead of the implicit intention. This outcome-bias in judgements has been previously studied through stories of ambiguous valences (good intention / bad outcome) which required a more substantial contribution of mental state reasoning (Margoni and Surian, 2016; Young and Tsoi, 2013; Zalla and Leboyer, 2011).

Several studies have focused on experiments involving MT scenarios based on the combination of intentions and outcomes (accidental / deliberate, good / bad), in neurotypical 
(NT) adults (Cushman, Dreber, Wang and Costa, 2009; Young and Saxe, 2011), young children (Cushman et al., 2013; Killen et al., 2011), and both autistic children (Grant et al., 2005) and autistic adults (Buon et al., 2013).

We must highlight the study by Grant and colleagues (2005), who assessed autistic, NT and moderate learning difficulties children with moral stories based on taking into account two factors: intentions (accidental or deliberate) and harmful outcomes (harm to a person or damage to property). Contrary to expectations, autistic children judged the agent's culpability on the basis of his/her intention, and they also stated that harm to a person was 'worse' than damage to property.

In a similar study, Rogé and Mullet, (2011), tested if autistic participants (age: from 7 to 36 years old, distributed in three groups of age) were able to judge the perpetrator of the act in MT taking into account two factors: intention (accidental / deliberate) and severity (not severe, medium and severe; i.e., severe consequences: hitting someone or breaking a radio). Although autistic participants did not perform as well as their chronological and developmental comparison group, they were able to use intent consistently and to recognise and distinguish more serious consequences from medium or not severe ones. In this line, the authors did not explore whether there was a difference in the severity factor when the mediator implied was personal or material (i.e., differences between stealing an object and someone who hurts another). In both cases, the victim would always be (emotionally) hurt differently: through damaging a property (material) or directly (personal). This is an aspect which it would be interesting to explore in depth because stories classified as having a personal mediator would imply more complex mental states inferences than those in which the mediator is material.

Rogé and Mullet's findings have been reproduced in similar studies related to morality in autistic adults. For example, in the study by Buon et al. (2013), no differences were found between groups of autistic and NT adults in questions about the suffering of the agent or the 
recognition of the cause of suffering. However, small difficulties were found in the autistic reasoning about intention and moral implication. The most interesting result of this work was related to severity, since autistic adults judged the agent who harmed the victim accidentally more severely than the NT group, even though when the intention was correctly assigned, as already mentioned by Moran et al. (2001). These results may show that, although the information about the mental states of the characters is inferred in the task, the autistic participants do have problems when it comes to using this information and generating a socially appropriate moral judgement (Zalla et al., 2011; Zalla and Leboyer, 2011). As explained above, this difficulty can be more salient in a faux pas situation, due to the important role of false beliefs and intentions (mental states) in its understanding.

To sum up, MT and FP tasks have usually been analysed separately in different studies with autistic population. In this sense, it appears relevant to find out if autistic people are impaired when it comes to understanding the agent's morality due to their poor ToM abilities in both tasks. Hence, the present research aims to use FP and MT tasks to determine whether the moral reasoning of autistic children differs depending on whether an action is deliberate (MT) or accidental (FP). In both tasks, the action is always considered 'bad', and it has a negative outcome for the listener / victim (feeling emotionally bad or sad), but on the one hand the intention was classified as deliberate (MT task) and on the other it was accidental (FP task).

\section{Hypotheses}

Three hypotheses derive from three relevant aspects based on the literature abovementioned: (1) ambiguous and unambiguous cases; (2) severity; and (3) intention- versus outcome-based judgements.

Due to the intergroup similarities in NT and autistic participants in Grant et al. (2005) and Rogé and Mullet (2011), Hypothesis 1 states that no significant intergroup differences will 
occur in the MT task (situations with unambiguous valences: bad intention / bad outcome) between autistic and NT children. Nevertheless, it is predicted that the FP test (situations with ambiguous valences: good intention / bad outcome) will be more complicated to solve for autistic children, and significant differences between groups could appear (Margoni and Surian, 2016; Moran et al., 2011; Zalla and Leboyer, 2011; Zalla et al., 2009).

Following the line of studies that focus on the severity of the situations and the agents involved (e.g., Grant et al., 2005; Rogé and Mullet, 2011; Zalla et al., 2011; Zalla and Leboyer, 2011), Hypothesis 2 posits that differences between groups are expected when the stories are divided by the mediator through which the damage is caused: personal mediator (getting revenge against someone, insulting a partner) / material mediator (stealing an object, disregarding a present). Those referring to a personal mediator are more difficult to judge for the ASD group, due to the complexity of mental states involved in these cases.

For Hypothesis 3, both groups are predicted to judge the agent (as good or bad) based on his/her intention, instead of the outcome (e.g., Grant et al., 2005; Rogé and Mullet, 2011). Thus, if the judgements are based on intention, both groups are expected to offer a more severe moral judgement of the agent (as bad) in the MT task (deliberate act) than in cases of the FP task (accidental act). In this sense, differences between tasks are predicted in intention (accidental-deliberate) and, as a consequence, the judgment of the agent (good-bad).

\section{Method}

\section{Participants}

A priori power analyses using $G^{*}$ Power indicated that a sample size of 26 participants per group would be adequate, with power set at $d=.80, \alpha=.05$ and a desired level of assurance of .80. This sample is comparable to previous studies conducted in the autism and ToM field (e.g., Grant et al., 2005, or Zalla et al., 2011). Sixty-two Spanish participants from mainstream 
schools took part in the present study. Their ages ranged between 84 and 145 months $(\mathrm{M}=$ 113.68 months $-9 ; 5, \mathrm{SD}=17.85)$; and the range of intelligence, based on participants' IQ, was between 80 and $130(\mathrm{M}=104.34, \mathrm{SD}=13.43)$.

Autistic group: it was formed by thirty participants ( 5 girls and 25 boys) previously diagnosed with level 1 ASD by specialised psychologists, using standardised and specific tests: ADOS (Lord, Rutter, DiLavore and Risi, 2008) and ADI-R (Rutter, Le Couteur and Lord, 2003).

NT group: it was formed by thirty-two participants ( 7 girls and 25 boys) matched by their chronological age and intelligence. No significant differences were found between groups on their age $\left(\mathrm{M}_{\mathrm{ASD}}=112.73(18.01), \mathrm{M}_{\mathrm{NT}}=114.56(17.96), t(60)=.40, p=.690, d=.10\right)$, and intelligence $\left(\mathrm{M}_{\mathrm{ASD}}=102.83(14.23), \mathrm{M}_{\mathrm{NT}}=105.75(12.69), t(60)=.85, p=.397, d=.22\right)$.

\section{Materials}

Intelligence Quotient (IQ) tests. To assess intelligence, Sattler's short adaptation (1992) of the WISC-III intelligence scale was administered (Wechsler, 1991). The total scores on this scale appeared in the report of the autistic children written by the psychologist or specialised neurologist (with a maximum of two years since the last administration). Due to the high correlation in the ASD group between the total IQ on the scores in the report and the scores on the short form (as found in the classic studies: Ryan, 1981; Sattler, 1992), the short form was administered to the NT group as a reliable estimate of the group's intelligence quotient, with the main objective of ensuring comparable IQ levels in both groups.

Advanced Theory of Mind Stories. A set of eight stories were presented by the research group using the E-prime multimedia program for computers with a touch screen for their forcedchoice responses. The stories were classified as:

- MT task: Four stories of intentional situations extracted from Garcia-Molina et al. (2016); 
- FP task: Four stories of accidental situations extracted from the faux pas task by BaronCohen et al. (1999), Spanish translation by Garcia-Molina et al. (2016).

Each story consisted of two vignettes (illustrations) with dialogues and narration. Both situations (MT and FP) followed the same structure: (1) speaker / perpetrator agent with his/her beliefs and desires - (2) action (bad) - (3) victim or emotionally affected agent (bad outcome). Situations differed in terms of the intent: agents in MT scenarios had a bad or selfish desire and they acted deliberately; however, agents in FP scenarios had a false belief and they acted accidentally. The decoding features involved in the MT and FP stories can be seen in Table

\section{4-13.}

In addition, each story was classified according to the severity of the harm caused by a person or an object as the mediator, the harm caused to an agent through a person being understood as more severe (e.g., getting revenge by hurting a person, see 7.5 and 7.3 Appendix; MT stories: the balloons, the soup stories; FP stories: the cook, the bathroom stories) than the damage caused to an agent through a material object (e.g., stealing the object; MT stories: the yo-yo, the ice-cream stories; FP: the plane, the apple pie stories).

Table 4-13

Decoding features involved in MT and FP stories.

\begin{tabular}{llllllll}
\hline \multirow{2}{*}{ Stories } & & Agent & & \multicolumn{2}{c}{ Action } & \multicolumn{2}{c}{ Outcome } \\
\cline { 2 - 7 } & Belief & Intention & Moral & Control & Moral & Emotion & Moral \\
\hline MT & True & Deliberate & Bad & Control & Bad & Sad & Bad \\
FP & False & Accidental & Good & Control/No & Bad & Sad & Bad \\
\hline
\end{tabular}

\section{Scoring of ToM tasks (MT and FP)}

Scoring (a) explained the scores of the recognition questions in general - Hypothesis 1 and 2. Scoring (b) was used only for Hypothesis 3 , in order to focus the comparison between groups in the questions related only in relation to intention and morality. 
a) Scoring in recognition questions.

At the end of each story, the same set of questions was asked for the MT and FP stories. The correct answer to each question is indicated in brackets after each question for the reader (Scoring a). The answers were analysed through questions related to:

(i) detection of the bad action or sentence: 'Did someone do/say something that they should not have done/said?' (the correct answer changes depending on the story);

(ii) recognition of the perpetrator / speaker agent: 'If so, who?' (it depends on the story);

(iii) intention: 'Do you think X wanted to make [the victim] feel like that?' (MT: yes / deliberate; FP: no / accidental);

(iv) morality of the agent: 'Is [the agent] good or bad?' (MT: bad, FP: good);

(v) morality of the action: 'Was what [the agent] did right or wrong?' (bad or wrong);

(vi) morality of the outcome / emotion: 'How does [name of the victim] feel?' (bad or sad). The answers were scored as: $0=$ wrong; $1=$ correct, with a total of 6 correct points per story, which means is a total of 24 points in MT stories and another 24 in FP.

b) Scoring for the comparison of intention and morality between MT and FP stories.

For comparing the relevant answers in MT and FP tasks only questions related to intention (iii) intention, and morality (iv) morality of the agent; (v) morality of the action, and (vi) morality of the outcome, were analysed. The questions (i) detection of the bad action or sentence and (ii) recognition of the perpetrator / speaker agent were excluded for analysis because both did not provide information about intention or morality.

The value for the FP task in questions (iii) intention and (iv) morality of the agent was reverse scored to compare the same valences between tasks. Each answer was scored as 0 or 1 point. Since the minimum score was 0 and the maximum was 4 in each task (four stories), an overall 
score closes to 4 would be bad or deliberate, while a score closes to 0 would be good or accidental. Answers were scored and expected as follows:

(iii) intention: 1 point $=$ Deliberate; $0=$ Accidental (in an MT story it is expected to be 1 ; in an FP story it is expected to be 0 );

(iv) morality of the agent: 1 point $=\mathrm{Bad} ; 0=$ Good (in an MT story it is expected to be 1 ; in an FP story it is expected to be 0$)$;

(v) morality of the action: 1 point $=\mathrm{Bad} ; 0=$ Good (in both an MT and an FP story it is expected to be 1);

(vi) morality of the outcome: 1 point $=\mathrm{Bad} / \mathrm{Sad}$; $0=$ Good $/$ Happy (in both an MT and an FP story it is expected to be 1).

\section{Procedure}

The study protocol was granted ethical approval from the university and Valencian Community Research Ethics Committee, and written informed consent was obtained from each child's parents or legal guardian. NT children were chosen from the same schools as the autistic children according to previously established criteria (IQ within the typical range and no diagnosis of comorbid psychiatric disorders, learning disabilities or injury involving the brain). In the following days, intelligence and ToM tasks were administered individually, in one or two sessions of approximately an hour and a half held in quiet rooms at the schools. All the tasks were administered in Spanish.

Two out of sixty-four participants were discarded because neither of them was within the established parameters for their inclusion (having an IQ $>80$ and passing a first-order false belief ToM task). 


\section{Data analysis}

The data were analysed using the statistical package SPSS 24 . The sample met the assumptions of the parametric analysis, according to the Kolmogorov-Smirnov homogeneity test. $T$-test for independent samples and $t$-test for related samples was used to establish differences within and between groups. For all the analyses, the $p$ value used to establish the statistical significance was .05.

\section{Results}

Differences in the MT and FP tasks between autistic and NT children.

In relation to hypothesis 1, the comparison between groups in the MT and FP tasks show that there are not significant differences and both groups performed better in the MT tasks (MT: $\left.\mathrm{M}_{\mathrm{NT}}=20.22, \mathrm{M}_{\mathrm{ASD}}=19.93, t(60)=1.73, p=.088, d=.44\right) ; \mathrm{FP}: \mathrm{M}_{\mathrm{NT}}=18.84, \mathrm{M}_{\mathrm{ASD}}=18.40$, $t(60)=.56, p=.577, d=.14)$.

When performance in both tasks is compared within groups, it can be observed that ASD group behave very similar in MT and FP tasks, although the lowest score was found in the FP $(t(29)$ $=.86, p=.397, d=.18$ ). Within the NT group, it can be seen - see means in the previous paragraph - that their performance was clearly better in the MT task, but with no significant differences in comparison to the FP task $(t(31)=1.86, p=.073, d=.44)$.

\section{Differences when the mediator is personal or material.}

With regard to hypothesis 2 , stories were divided into personal mediator and material mediator, according to the severity of the harm. Significant differences between groups are only observed in the MT stories classified as personal mediator $(t(29)=2.66, p=.010, d=$ .67). See Table 4-14 
Table 4-14

Differences between groups (t-test) divided by personal or material mediators.

\begin{tabular}{|c|c|c|c|c|c|c|c|c|}
\hline Mediator & \multicolumn{2}{|c|}{ ASD (30) } & \multicolumn{2}{|c|}{ NT (32) } & \multirow[b]{2}{*}{$\mathrm{t}$} & \multirow[b]{2}{*}{$p$} & \multirow[b]{2}{*}{$d^{a}$} & \multirow[b]{2}{*}{$\mathrm{SE}(r)^{\mathrm{b}}$} \\
\hline MT & $\mathrm{M}(\mathrm{SD})$ & Range & $\mathrm{M}(\mathrm{SD})$ & Range & & & & \\
\hline Personal & $9.23(1.74)$ & $5-12$ & $10.37(1.64)$ & $6-12$ & -2.66 & .010 & .67 & .32 \\
\hline Material & $9.70(1.74)$ & $6-12$ & $9.84(1.87)$ & $3-12$ & -.313 & .756 & .07 & .03 \\
\hline $\mathrm{FP}$ & & & & & & & & \\
\hline Personal & 8.57 (1.89) & $5-12$ & 9.09 (1.98) & $5-12$ & -1.07 & .290 & .27 & .13 \\
\hline Material & $9.83(1.42)$ & $7-12$ & 9.75 (1.67) & $6-12$ & .212 & .833 & .05 & .02 \\
\hline
\end{tabular}

$\mathrm{MT}=$ Moral judgements task; FP = Faux pas task; $d^{a}=$ Cohen's $d ; \mathrm{SE}(r)^{\mathrm{b}}=$ Size Effect

\section{Differences between groups in intention and agent's morality.}

Related to hypothesis 3 , intention and morality responses were compared in both groups for MT and FP tasks.

The analysis of the paired $t$-test revealed several significant differences between MT and FP stories in the NT group regarding different aspects: deliberate intentions $\left(\mathrm{M}_{\mathrm{MT}}=2.13, \mathrm{M}_{\mathrm{FP}}=\right.$ $1.03, r=.050 ; t(31)=4.16, p<.001, d=.85)$, morality of the agent $\left(\mathrm{M}_{\mathrm{MT}}=2.84, \mathrm{M}_{\mathrm{FP}}=2.03, r\right.$ $=.000, t(31)=4.10, p<.001, d=.58)$ and morality of the action, understood as "bad" $\left(\mathrm{M}_{\mathrm{MT}}=\right.$ 3.91, $\left.\mathrm{M}_{\mathrm{FP}}=3.59, r=.030, t(31)=2.40, p=.023, d=.49\right)$.

In the ASD group, significant differences were only found in deliberate intentions $\left(\mathrm{M}_{\mathrm{MT}}=1.83\right.$, $\left.\mathrm{M}_{\mathrm{FP}}=.90, r=.004, t(29)=4.26, p<.001, d=.77\right)$ and morality of the action $\left(\mathrm{M}_{\mathrm{MT}}=3.83, \mathrm{M}_{\mathrm{FP}}\right.$ $=3.57, r=.024, t(29)=2.50, p=.018, d=.50)$.

The variables related to the FP stories were always judged as 'less bad' or 'less deliberate' than those of the MT stories. 

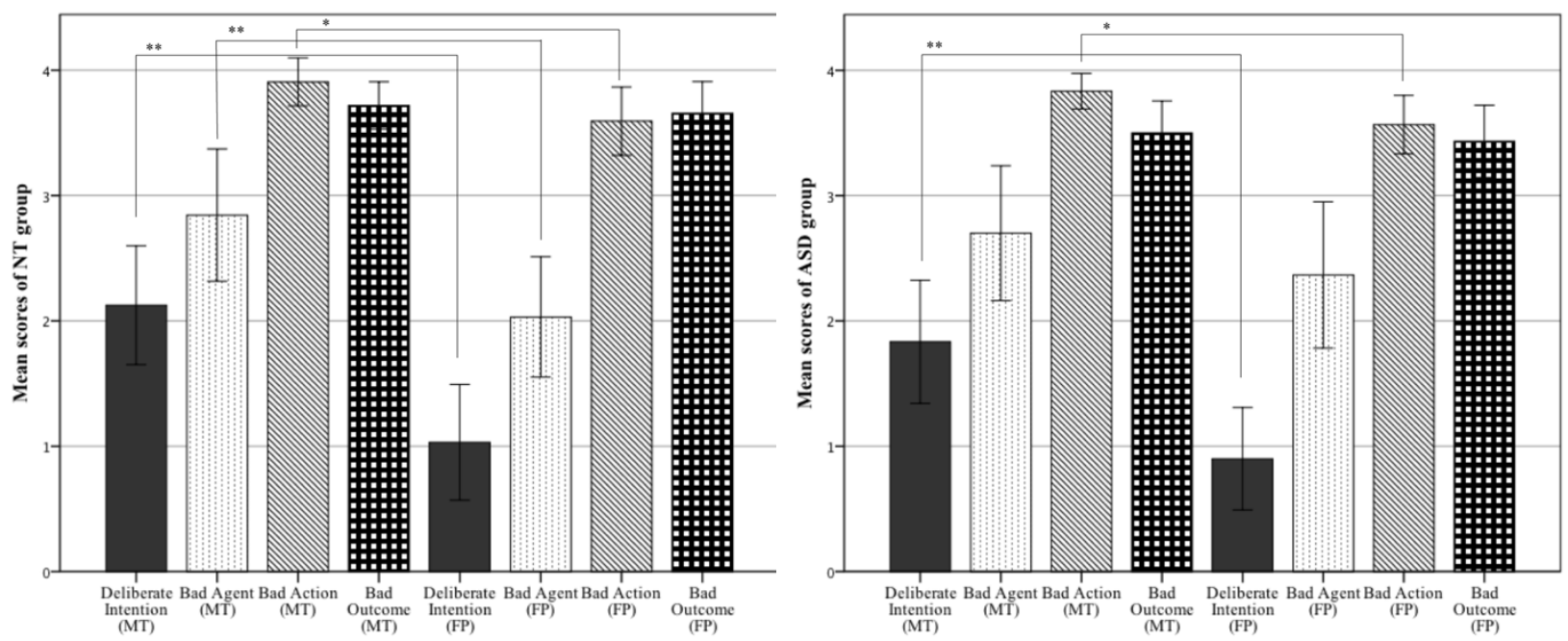

Figures 4-7 and 4-8. Mean scores of the NT (2) and ASD (3) groups according to their judgements on: intention, agent, action and outcome in MT and FP tasks. See scores in section b) of Material: Scores on the ToM task (maximum of 4 on badness / deliberate).

In general, Figures 4-7 and 4-8 show similar scores. Taking as a reference the fact that the higher they score, the more 'deliberate' the intentions are and the worse (bad) the agents / actions / outcomes are, it is easy to see the relationship between bad action and bad outcome in both groups (the action and outcome of the situations were judged as bad). However, it is necessary to perform a more detailed analysis to determine whether intention has more or less weight in the judgement about the agent's morality:

Regarding the NT group in the MT task (Figures 4-7 and 4-8) the recognition of the intention seems confused, not being understood as accidental or as deliberate $(\mathrm{M}=2.13)$. Yet, the character is judged as bad $(\mathrm{M}=2.84)$. In the FP stories, the intention is quite correctly understood as accidental $(\mathrm{M}=1.03$, close to the minimum $=$ accidental $)$. The agent's moral judgement denoted that the intention could have had an influence because the score is not understood as being bad, but neither he/she is seen as $\operatorname{good}(\mathrm{M}=2.03)$.

Regarding the ASD group (Figure 4-8), in the MT stories, the intention is not understood as deliberate $(M=1.83)$. Instead, the morality of the agent approaches being judged as bad more than as $\operatorname{good}(\mathrm{M}=2.70)$. In the FP stories, the intention was correctly understood as accidental, 
due to its value being close to $0(\mathrm{M}=.90)$, but the morality of the agent tends to be understood as bad more than as $\operatorname{good}(\mathrm{M}=1.63)$.

In both the NT group $\left(\mathrm{M}_{\mathrm{MTaction}}=3.91, \mathrm{M}_{\mathrm{MToutcome}}=3.72, \mathrm{M}_{\mathrm{FPaction}}=3.57, \mathrm{M}_{\text {FPoutcome }}=3.43\right)$ and the ASD group $\left(\mathrm{M}_{\mathrm{MTa} \text { ation }}=3.83, \mathrm{M}_{\mathrm{MToutcome}}=3.50, \mathrm{M}_{\mathrm{FPaction}}=3.57, \mathrm{M}_{\mathrm{FPoutcome}}=3.43\right)$ the action and outcome were judged as bad in the MT and FP stories.

\section{Discussion}

The present study was designed to investigate differences between groups (ASD - NT) according to their ability to interpret intentions and judge morality, in two types of deliberate and accidental scenarios (MT and FP), both based on social situations of everyday life. First, it was hypothesised that autistic participants would be able to respond to the MT task as well as their comparison group. However, in the FP task significant differences could appear. Second, it was predicted that significant inter-group differences could be found in the stories classified as involving a personal mediator. Third, it was expected that both groups could base their judgements on intention instead of outcomes. These predictions were partially confirmed. The ASD and NT groups obtained very similar scores in both tasks, although with subtle difficulties in the ASD group regarding the judgements of MT when the mediator was personal and also the FP agent's morality. Several important aspects of this research are specified in more detail below.

Within hypothesis 1, it should be noted that autistic children responded in a similar way to their comparison group (NT), thereby confirming the results of other studies similar to the current work (Grant et al., 2005; Moran et al., 2001; Rogé and Mullet, 2011). In relation to hypothesis 1 , although no significant differences were found between MT and FP stories, both groups obtained the lowest scores in FP task. This finding is in agreement with the established knowledge that accidental intention is more complex to understand and appears later in child 
development (Cushman, 2013), due to the need to understand the false belief and intentions of the agent involved in the stories (Baron-Cohen et al., 1999; Zalla et al., 2009).

In the present work, most of the intentions of the FP stories were correctly identified as 'accidental'. As a noteworthy point, the agent's intention question of the present study was linked to the plan/desire to emotionally hurt another person, as stated in previous studies (Banerjee and Watling, 2005). Thus, participants from both groups were aware of the listener's emotions. In other studies, this question was associated only with desires or motives (for more examples see Buon et al., 2013, or Zalla et al. 2009).

With regard to the Hypothesis 2, when stories were classified according to the mediator of the harm or damage (person or object), significant differences were found between groups only in the MT stories. As expected, the fact that the mediator through which the action occurs is directed towards another person (for example, having the desire to take revenge on a person) may be mentally more complex to interpret than when the action is directed towards an object (for example, having the desire to obtain an object belonging to someone else and to steal the object). In addition, in the case of the person as a mediator, the process usually involves more painful harm than in the case of an object, and it also seems to imply the concept of reversibility (objects can be replaced, but hurting someone cannot be undone; Grant et al., 2005).

The fact that the mediator is personal implies two understandings of mental states. First, there is the understanding of mental states of character $\mathrm{X}$ (the perpetrator of the action), with specific desires and beliefs. Second, there is the understanding that character Y, who is affected by the action, also has a mind and does not know the information about X's desires. Regardless whether the cases are ambiguous or unambiguous, to resolve them correctly, all this mentalstates information has to be integrated, which shows the complexity that autistic individuals could find in both situations analysed (Moran et al., 2001; Zalla et al., 2011; Zalla and Leboyer, 2011). This explanation is confirmed by the fact that both groups responded very similarly to 
the stories classified as involving a material mediator, in which the inference of mental states is less complex.

Regarding the hypothesis 3, both groups obtained very similar scores in the judgements of intention and morality, as can be observed visually in the graphs. Thus, autistic children in this study understood the intention (as happened in previous studies, e.g., Grant et al., 2005). However, they rated the agent as 'bad' even when the intention was understood to be accidental (Buon et al., 2013; Moran et al., 2011, Zalla et al., 2009). This fact is corroborated by the nonsignificant differences between the MT and FP stories regarding the morality of the agent in the ASD group. As expected, important differences did exist in the NT group, since the agent of the MT stories was judged more severely ('worse') than in the FP stories. However, in the ASD group, the morality of the agent tended towards 'bad' (even in FP), and such responses may be influenced by the bad outcome (character Y was hurt) even when the agent's intention was understood. This discrepancy suggests that autistic individuals could have problems with ToM capacity, since they show outcome-based judgements when cases were ambiguous: the agent is bad because the outcome was bad (Margoni and Surian, 2016). Another possible explanation is that autistic individuals have difficulties when it comes to integrating both paths required in faux pas (paths 1 and 2 of the model of intention for the FP, see Figure 4-6) or changing one path to another. On the one hand, there is the information and the mental states attributed to the speaker. On the other hand, there is the information that we as observers have. It is well established that autistic persons have problems in social flexibility and inhibition; namely, to inhibit the information from the first path in order to be flexible and integrate the information from the second path. For this reason, autistic individuals could have problems to make a moral decision based on, first, the false belief of the speaker committing a faux pas path 1 - or, second, the accidental harm - path 2 (Figure 4-6). 
Autistic participants could remain on one of the two paths, understanding the main character's false belief or the intention. Therefore, they could have difficulties processing all this information, making a correct decision regarding the agent's morality.

\section{Limitations}

Some shortcomings regarding this study need to be acknowledged here. The first issue concerns the small sample size of our population. The novelty of this work lies in the fact that, as far as we know, no other study has used these two types of tasks (MT - FP). However, moral dilemmas and Faux Pas task have been widely administered. Although this work has focused on distinguishing intentions (accidental and deliberate), this distinction has not been made in the outcomes (good / bad), and there are no good results in any of our tasks. For future studies, neutral or good outcomes might be considered in order to see whether the results obtained (e.g., agent's morality) are affected by the outcome of the action. Similarly, it may also have been useful to include scenarios with negative outcomes with no-intention, namely the negative outcome is not the fault of any individual (e.g., a scenario where two children are happily making a snowman but, suddenly, the sun comes out and the snowman melts and becomes water). Finally, it would have been interesting to collect responses using Likert scales ('Who do you think was guiltier?', 'How guilty?') or collect verbal information related to more variables, e.g., punishment ('Would you punish X?'). In this sense, we could control ceiling effects, due to it seems that some aspects are not sensitive enough (i.e., action-morality) to individual differences. While whether we would use Likert scales for responses, findings could be more accurate than in dichotomous forced-choice answers.

\section{Conclusions}

In sum, the present findings show that autistic children do not have severe difficulties in reasoning adequately about judgements of moral tasks. The subtle differences found for 
beliefs or intentions when comparing it to the NT group can shed some light on the difficulty that autistic people can face in their social relationships which can be more complex in real life: with movements, gestures, body language or gazes. Finally, it is relevant to mention the support of the explanation in Figure 4-6, adapted from previous works, which contributes in a visual way to break a faux pas down into aspects that play an important role in its understanding (in terms of intentions and morality). This decoding allows the professional in education and psychology to understand the subtle difficulties that autistic individuals encounter in similar tasks as well as in their interpersonal relationships. 
Capítulo 5 / Chapter 5 General Discussion and Conclusions 


\subsection{General Discussion and Conclusions}

Little research has been carried out on the moral reasoning of situations which differ from intention (e.g., negative intention - Moral Transgressions and good intention - Faux Pas), specifically in level 1 autistic individuals whose native language is Spanish. Also, another aim was to investigate which factors could influence the resolution of these social scenarios (e.g., an awkward comment). To address this issue, the present thesis incorporates different studies focusing on the study of these complex ToM skills. Moreover, these abilities have been assessed through tasks designed to be similar in their structure (both with bad outcomes), but different in their intentionality. In this line, some subtle differences between groups (ASD NT; or lower IQ - higher IQ ASD) and tasks were expected.

According to the aims set out at the beginning of this thesis, the results of the four studies presented in Chapter 3 are discussed in the following paragraphs. A summary of these aims can be found in Box 1, followed by the numbers of the studies addressing them.

Following the structure of Figure 3-1(a) and 3-1(b), page 57 (which shows the construction of the four studies following the aims cited), the empirical findings of the different studies have been analysed. Objective 1 aimed to study the differences between ASD and NT groups in deliberate situations (Study 1) and accidental situations (Study 2 and Study3). Objective 2 intended to examine the possible influences of other variables (e.g., intelligence, working memory and modality of presentation) on the resolution of advanced ToM tasks by autistic children (Study 2 and 3). Finally, Objective 3 aimed to find out whether there are differences in the understanding of everyday situations when actions are accidental or deliberate (Study 4). 
Box 1. Summary of objectives

5.1.1. Discuss the differences between groups in deliberate and accidental situations

5.1.1.1. Differences between groups in deliberate situations - moral transgressions (Study 1)

a) Structure of the story. Non-ambiguous valences

b) Working memory influences in moral judgements

c) The verbal weight and the abstraction level

5.1.1.2 Differences between groups in accidental situations - faux pas (Study 2 / 3)

- Differences in Study 2

- Differences in Study 3

- Two perspectives for understanding a faux pas

5.1.2. Recognise whether certain factors influence the understanding of a faux pas

5.1.2.1 Intelligence influence (Study 2 / 3)

- Intelligence in Study 2

- Intelligence in Study 3

5.1.2.2 Working Memory influence (Study 3)

5.1.2.3 Modality of presentation influence on complex mentalistic reasoning (Study 3)

5.1.3. Analyse the differences between Moral Transgressions and Faux Pas tasks for both autistic and NT participants (Study 4)

\subsubsection{Discuss the differences between groups in deliberate and accidental situations.}

In order to fulfill the first objective, it has been necessary to analyze the between-group differences in Moral Transgressions and Faux Pas tasks. These conclusions have been collected throughout the four studies of this thesis. More specifically, Studies 1 (moral transgressions), and 2 and 3 (faux pas) have been conducted.

As understanding of mental states is always important in terms of moral reasoning, both types of tasks - Moral Transgressions and Faux Pas - were considered here as advanced ToM tasks and therefore, difficult for autistic individuals. First, unambiguous cases will be considered (moral transgressions). Secondly, ambiguous cases (faux pas) will be analysed more in detail. 


\subsubsection{Differences between groups in deliberate situations - moral transgressions}

\section{(Study 1)}

Study 1 compared the moral judgement performance of 60 children (with and without autism), using a novel verbal moral judgement task. This task focused on three different scenarios for children which were morally unacceptable (e.g., lying). Five measures were assessed with forced-choice responses (Yes/No; Good/Bad), whilst the other two were categorised according to the quality of the response (categories: (i) related to mental states; (ii) descriptions; (iii) literal reiterations; (iv) inappropriate) and the low to high complexity of the information (ToM content and No-ToM content).

As cited above, in this first study, scenarios and questions followed a traditional format for this type of task, which is verbal modality (information was auditory and answers were collected orally). Focusing on the differences, which is the point that this first objective concerns, it should be noted that no differences were found between groups in forced-choice questions related to (1) detection of transgression, (2) identification of the perpetrator, (3) emotions, (4) perpetrator morality, (5) wrongness of the act, with both groups (ASD - NT) responding in a similar way.

However, when scenarios were compared (e.g., lying, stealing and breaking), intergroup differences were found in the story about lying, in which scenario it was necessary to understand more aspects related to mental states - and the double morality that emerged from lying and blaming another person (two bad acts). In this sense, as detailed in some research based on the study of lies (see Spence et al., 2003, or Talwar and Lee; Talwar et al., 2012), a lie exists if the speaker causes a false belief in the mind of the listener with the intention of deceiving. As in the story, the child (speaker) knows something that her mother (listener) does not know, allowing the child a certain degree of control (deliberate action). Also, the observer may take into account that, as a dog cannot speak, the moral strategy of the child is doubly bad. As a consequence that the child blames the dog, the girl will be innocent, the mother will put the blame on the dog due to a lie (false belief), and the dog will be emotionally hurt.

Furthermore, an important finding of the first study is reflected in the verbal justifications of the morality of the act and the intention of the agent. Verbal justifications were classified in established categories, and differences appeared significant between groups in five out of the six verbal justifications. Moreover, upon reflection, this pattern tends to repeat itself 
throughout the different studies when they are contemplated. Regarding the differences in morality, verbal reasoning of NT children was classified into mental states (category i) and descriptive (category ii) categories. In the autistic group, the classifications were more heterogeneous, between descriptive (category ii) and inappropriate (category iv) categories. In the differences about intention, the responses of NT children were based on the description of the actions, and they used mentalist arguments as well (categories $\mathrm{i}$ and ii). The autistic group focused their justifications on the description of the event (classified as category ii, descriptive).

Intergroup differences were more marked when both groups reasoned about the morality of the act rather than the intention, appearing more complicated for autistic children to judge the morality of an event. Actually, as detailed in the Theorical Framework section, reasoning about morality implies a series of elements to be taken into account, among which the intention is implicit: one needs to contemplate the intention for judging an event (following Cushman's model, 2015).

These intergroup differences based on ToM capacity can be clearly observed in the Figures 4-3 and 4-4 which graphically show the classification of the justifications into ToM content and No-ToM content categories. These differences could explain what the studies analysed showed (e.g., Grant et al., 2005) that considering an appropriate answer is linked to the use of mental state terms in the individual's explanation (Bishop and Norbury, 2002). This kind of differences between groups has been repeated throughout studies. Some influences were analysed in order to know what variables could affect autistic people in their reasoning of everyday situations. These factors were not tested in Study 1; however, these conclusions were used to build the subsequent studies. The influences were the following:

\section{a) Structure of the story. Non-ambiguous valences}

The structure of stories is highlighted in Study 1, based on unambiguous valences (negative intention / negative outcome). More deeply, the structure could be analysed following Cushamn's model (2013): Bad desire (selfish desire) - Bad intention (action is planned) - Bad action (wrong act) - Bad outcome (hurting someone emotionally).

In Study 1, as the intention and the outcome were not ambiguous, children appear to find it easier to understand the bad action because they may have linked it to the bad consequences or the victim's emotion, as commented in Margoni and Surian's study (2016). Likely, they do not link it to the agent's mental states, as demonstrated in the reasoning section. 
This variable can be better analysed in similar everyday life-based studies with ambiguous valences: good intention / bad outcome, as it will be contemplated in the next section (5.1.2).

\section{b) Working memory influences in moral judgements}

WM is another factor that should be considered as a possible influence in moral transgressions, as cited in the conclusion section of Study 1. Indeed, Study 3 was constructed based on the conclusions of Study 1, related to the possible influence of WM in advanced ToM tasks.

Study 1 mentioned that WM is required in peer-to-peer dialogues and other real-world social situations, but also this capacity is important in laboratory tasks, and this capacity could have an influence in the moral judgements of children.

Research mentioned in Study 1, is the work of Moore et al. (2008). In this study, individual WM differences predicted that participants with higher WM capacity made more appropriate judgements than did subjects with lower WM capacity (i.e., participants with higher WM contemplated factors as inevitable death for deciding the appropriate judgement). Also, WM affected consistency with which people made similar moral judgements across personal dilemmas (i.e., serious bodily harm or death to another person or persons which the perpetrator directly initiates). It is important to consider another study, Greene et al. (2001, 2004), which found that personal dilemmas engage brain regions involved in emotion (e.g., amygdala, posterior cingulate / precuneus). However, impersonal dilemmas - dilemmas that do not meet the criteria mentioned in personal dilemmas - activated areas involved in deliberative reasoning and working memory (e.g., middle frontal gyrus, bilateral parietal lobe).

Stories in Study 1 (i.e., lying, breaking and stealing) would be classified as impersonal (Greene et al., 2001, 2004; Moore et al., 2008). However, emotion would be involved as well, due to the importance in the questions and scenarios created deliberately by the group. For example, in the Dog story (lying), the dog cries and the mother was disappointed; in the Car story (breaking), the girl was surprised by the selfish behavior of her friend; in the Sandwich story (stealing), the girl was disappointed because she trusted her friend. Also, in Study 1 there were questions related to emotions, such as 3) emotion triggered in the victim. Thus, emotion is important in the moral scenarios of the current work as well. Furthermore, in accordance 
with Moore et al. (2008), WM capacity only predicts the judgements in personal dilemmas, which are more related to emotion (Greene et al., 2001, 2004).

WM capacity would be relevant to interpret the moral scenarios presented and must be controlled for in future research. For this reason, it was contemplated in Study 3. WM could affect the responses of autistic children in the different scenarios closely classifiable as impersonal, and the emotions involved could intensify the difficulty to the scenarios presented, as autistic children may link the understanding of moral scenarios to the victim's emotion (Moran and Surian, 2016).

\section{c) The verbal weight and the abstraction level}

When someone verbally reasoned about a moral scenario, the most mentalistic difficulties referring to the perpetrator can be appreciated, such as details that have been misinterpreted or repetitive descriptions. For this reason, verbal responses are important.

In the current work, verbal responses were needed in some studies (i.e., Study 1 and 2 were carried through open questions). According to this rationale, the second objective will be focused on the possible differences between modalities of presentation of the tasks (purely verbal, purely visual, mixed scenarios).

In Study 1, scenarios and responses were completely verbal (narration, dialogues, questions and responses) and the verbal justifications of autistic children were more related to descriptions than mental states. Verbal representations implied a high-level of abstraction of reasoning, which could also play an important role in understanding and judging.

According to construal-level theory, the same objects and events may be represented (construed) at multiple levels of abstraction (Liberman \& Trope, 2008; Trope \& Liberman, 2010). Co-workers, Amit, Algom, and Trope (2009), proposed that verbal representations facilitate more abstract, high-level construals, whereas visual representations facilitate more concrete, low-level construals.

As it is well-documented, autistic children may have problems in abstract or symbolic representations, and in some cases, imagination appears to be limited (structured-interviews as ADI-R or observation schedules as the ADOS contemplate these items).

The verbal weight (and its difficulty for autistic children) will be seen in objective 2 (specifically in Study 3) as well, as a possible influence in faux pas stories. 


\subsubsection{Differences between groups in accidental situations - faux pas (Study 2 and}

3)

Two studies specifically focused on faux pas situations, ambiguous cases based on positive intention and negative outcome. These studies are Study 2 and Study 3.

In Study 2, both groups had a diagnosis of ASD, and the sample was divided by their IQ. In contrast, in Study 3, the sample was divided by diagnosis (autistic and NT children). These studies are analysed in more detail here:

\section{Differences in Study 2}

Focusing on Study 2, the ToM task presentation used was mixed, containing both images and audio. As expected, Group 1 (high-average IQ) responded better than Group 2 (low-average IQ). The two groups differed significantly in the total score of the Faux Pas task (forced-choice questions). After analysing the answers of forced-choice questions in more detail, significant differences were found in the (ii) recognition of the faux pas speaker, and the (vii) ignorance of the information. It is highlighted that this is the only study in which there are intergroup differences when forced-choice responses are analysed, whose sample is divided into two groups, both with a diagnosis of ASD.

The difficulties in the (ii) recognition of the faux pas speaker on part of Group 2 (low average IQ), would be related to the speaker's belief and what he/she says. That is, the lower level group would not understand the awkward statement, but contrary, would judge the listener's comments inappropriately and deliberately. In the Apple Pie faux pas story (see 7.3 Appendix 3), one can observe it more clearly. Children of group 2 would tend to point out that the character who said something awkward was the one who said that she had made an apple pie - the victim / listener - misunderstanding that she did it on purpose, and not the one who said he did not like apple pies - the speaker - due to ignorance of what the cake was made of.

Thus, it is suggesting that both groups differed in the question about ignorance as well, since this variable is related to the false belief that the character-speaker has (due to lack of information). False belief is of great importance for understanding a faux pas and possibly is the more mentalistic question in the task. The responses of the low-average IQ group would be influenced by the information that they as observers do have (true belief), in contrast of knowing the speaker's mental states (speaker's false belief). 
As regards to the verbal justifications of Study 2, Group 1 provided significantly more adequate answers than Group 2. Even so, both groups found difficulties, and their explanations were classified as the (1) repetition of the statement. This fact was related to the echolalia or literal repetition of part of the dialogue, due to the parroting effect of the autistic children.

In addition, "over-guilt" and deliberate intentionality were denoted in both groups' explanations. This pattern was repeated throughout the studies, an observation that corresponds with Zalla et al. (2009) faux pas findings: the descriptions were malicious and the terms used were not appropriate to the character intention in the ASD group. In Study 2, Group 2 (lower IQ) gave more inappropriate responses in this sense - shorter and offensive sentences - than Group 1.

In reference to this pattern of judgments (i.e., judging a character more maliciously or intentionally a character), more detailed information would need to be researched, and for this reason, Study 4 was constructed. In fact, as shown in objective 3, the same judgments were repeated in this work.

\section{Differences in Study 3}

Focusing on Study 3, participants were divided into ASD and NT group, and the ToM task presentation was very important, because there were three modalities of presentation: visual, verbal and mixed. Differences between groups were found when the presentations of the stories were purely visual and verbal, with lower scores from the autistic group. When modalities were compared to the ASD group, the verbal presentation of the task was considered as the most difficult for them.

Again, as mentioned in the Study 1, in Study 3 the high level of abstraction of the task could have affected the story comprehension in the autistic group. In these verbal scenarios, high imagination and symbolic demands were required for the total comprehension of the story.

As both Study 2 and 3 were based on faux pas stories, it is necessary to conduct a deeper analysis for understanding where the differences could have originated.

\section{Two models for understanding a faux pas}

To understand a faux pas, the present thesis provides, as a novelty, two figures in the Theoretical Framework (Figure 2-5 and Figure 2-6; pages 46 and 48). There would be two ways of approaching the decomposition of a faux pas: a) from the analysis of the situation, which is 
usually found in previous studies - demonstrated in Figure 2-5; and b) from the analysis of the two perspectives of people involved (what the speaker knows and what we as observers know) - Figure 2-6.

In a general way, in a faux pas situation, the belief of reality is false, and therefore, it affects the action (comment) which becomes accidental. Successively, this action causes an unwanted outcome in the listener (commonly, the listener is hurt emotionally).

However, the faux pas can also be illustrated and deconstructed via two pathways, thus observing the importance of the belief that forms the intention of the commentary in the mind of both, the speaker and the observer. These beliefs would differ according to the knowledge of reality that the person has (false / true). For this reason, the observer should be able to recognize his/her own perspective and the mental states of the other characters of the story. The most important perspective would be the speakers' mental states (false belief) and the recognition of the two types of actions that compose the faux pas: normal comment (action 1 - speaker perspective) and offensive comment (action 2 - observer perspective). That is, the observer should recognise that he / she knows the truth, but the speaker does not know it because he / she has a false belief of the reality.

This way of recognising the two paths has not been analysed before, and after the model presented in this thesis a faux pas could be understood in a clearer and more structured way. What happens in the mind of the speaker and the observer has been explained from a more detailed analysis to make it more understandable for autistic people, who may process information in a different way, and this structure may be helpful for them.

Most likely, the two models deconstructing a faux pas recognise the false belief as, probably, the main requirement to completely understanding a faux pas. For this reason, it is not surprising that in the predictive analyses of Study 2, the False Belief task correlated with and could explain the variability in the Faux Pas test. False belief is understood as what it is, the false or true belief of our or others' reality, and it depends on the perspective (a reasoning based on ToM ability).

It seems obvious that the comprehension of others' belief (and our own belief) is understood from the ToM competence. However, some researchers investigated whether False Belief tasks are actually measuring ToM ability (Bloom \& German, 2000; Killen et al., 2016). As Killen and colleagues (2016) stated, prototypical false belief tasks measure one's access to knowledge about the physical world, such as in the case of the change of location task, in which 
design there is limited social content: a) no relationship between the characters $\mathrm{X}$ and $\mathrm{Y}$ (e.g, if they are friends or strangers); b) the relationship between the object and the person who moves it is not explained either; c) no explanation of the intention or motivation that leads the character that manipulates the object to put it in another place, d) no recognition of the emotions caused by the action to the character (Killen et al., 2016).

For these reasons, in the current thesis a well-known or typical False Belief task (e.g., Sally and Anne task) was not used. The False Belief task used was extracted from De Villiers and De Villiers (2012) for different reasons. First, it was completely visual, because it was created originally for deaf children, and the information was clearly structured in vignettes and very understandable. Second, the characters seem to be relatives and the 'object' is a pet. See 7.6 Appendix 6.

In addition, following Killen and team's work, false belief competence was measured also with advanced ToM tasks. Therefore, the main advanced ToM tasks presented here (Moral Transgressions and Faux Pas) contemplated all the points mentioned above, including relationship, intention and emotion. Questions in the advanced ToM tasks were related at mentalist aspects as well. Concretely, the observer may understand the characters' perspective directly, such as intention, emotions or judgements questions. In the case of the Faux Pas, the characters' perspective in the ignorance question is very complex. Actually, observers may understand the second path of a faux pas, as if they could read the characters' minds.

Above all the questions, the ignorance question deserves more attention due to, in the seminal faux pas study, has also be named as 'False Belief Question' (Baron-Cohen et al., 1999). This is the only question that the participant must fight against the information that he / she knows from the information that the character-speaker knows, for a correct answer.

The Faux Pas test seems to be one of the most complicated tasks, conformed by two ambiguous valences. Furthermore, moral aspects were included in the scenarios and questions for analysing the judgements of a faux pas in terms of intention, action and agent, according to Buon et al. (2013) discussion.

Due to these possible influences and the conclusions of the first two studies (the weight of the intelligence, the level of abstraction of verbal and visual stimuli or the WM influence), it was decided that Study 3 would be carried out with the aim to find these possible influences for understanding Faux Pas test. 


\subsubsection{Recognise whether certain factors influence the understanding of a faux pas}

The results of this second aim are considered the most useful in terms of intervention or construction of advanced ToM tasks, in order to facilitate the comprehension of this type of situation. Study 2 and 3 tried to recognise the possible factors that can influence in the resolution of an advanced ToM task, as the Faux Pas test.

The section 2.1 will be analysed based on Study 2 and 3 .

The sections 2.2 and 2.3 were answered based on Study 3 results.

\subsubsection{Intelligence influence (Study 2 and 3)}

\section{Intelligence in Study 2}

The main goal of Study 2 was to understand whether ToM ability could be related to intelligence. It seems that in Study 2, the IQ could have an influence in the resolution of the Faux Pas test: for example, the higher cognitive level, the better the score in the task.

Different studies have linked intelligence to various areas of life for autistic people, such as school and academic success (Eaves \& Ho, 1997, Mayes \& Calhoun, 2003), adaptive behavior or personal independence (Charman, Pickles, Simonoff, et al., 2011; Duncan \& Bishop, 2011). Happé's work (1994b) established a significant relationship between the problems in mentalist tasks and the difficulties in the Comprehension subtest of the Wechsler scale in autistic adolescents. Contrary, this relationship was not found for the performance of the Blocks - a manipulative test.

One of the most fundamental bases of hypothesised as to why verbal IQ could correlate with ToM is because ToM tasks are mainly verbal tasks, and verbal IQ involves, language ability (Happé, 1995; Jenkins \& Astington, 1996; Sullivan \& Ruffman, 2004; Tager-Flusberg \& Sullivan, 2000; Wang \& Su, 2006).

In Study 2, IQ full-scale and Vocabulary subscale predicted - as well as False Belief the performance in Faux Pas test (no verbal explanations were contemplated). Importantly, our results presented here supported the findings of Wang and $\mathrm{Su}$ (2006), due to crystallised ability (the ability to use skills, knowledge, and experience) correlated with Faux Pas test. For measuring crystallised intelligence, in Wang and Su (2006) study, the Vocabulary subtest of the Wechsler scale was administered, as in Study 2.

However, it should be noted that in Study 2, the Faux Pas test presentation was mixed (included illustrations) and some reminders were left on the screen (such as the visual story). 
Contrary, in Wang and Su (2006) study (as well as in Stone et al., 2003), the stories were printed on separate pages and participants were required to answer the question on a blank piece of paper, remembering what happened in each scenario. One of the questions was answered orally, as in Study 2. Possibly, verbal WM could be influenced in the responses of the participants of Wang and Su (2006), and for this reason, as commented in Study 1, we decided to analyse the WM in Study 3. Below (5.1.2.2), WM prediction will be analysed in more detail.

Importantly, this confirms the relevance of the effect of linguistic tasks in performing tests about social situations linked to interpersonal beliefs, intentions or desires. Grammatical and pragmatics comprehension would be an important part for understanding a faux pas, even so when verbal explanations are not contemplated.

In addition, the model excluded performance IQ (Blocks subtest) for predictive analyses, as in Happé (1994b). Conclusions of Study 2 consider the explanation of Happé and Frith (2006) for this rejection based on Weak Central Coherence theory (WCC). Focusing on this different way to process information, the authors would suggest that autistic individuals would need less effort in the capacity to segment elements, as it could otherwise typically be seen in their Blocks subtest high scores.

However, it should be mentioned that the whole sample of Study 2 had a large range of IQ full-scale: from 70 to 120 (the sample was divided into two groups depending on their IQ full-scale after the predictive analyses). For this reason, with the purpose of knowing more about the effect of IQ in the Faux Pas test in individuals with and without an autism, Study 3 was designed.

\section{Intelligence in Study 3}

In Study 3, IQ full-scale was controlled between groups: autistic and NT children. Importantly, only some significant correlations were found within the ASD group between visual and mixed Faux Pas test and IQ full-scale, which was in the opposite direction of what we concluded in Study 2: the importance of IQ when ToM tasks are mainly verbal, and the important part of auditory information in a faux pas. On the other hand, no correlations were found within NT group. 
In addition, an important effect found in the prediction analyses was when IQ scores were similar between groups of average IQ, the regression model excluded the IQ from all the predictions.

Therefore, when IQ scores are on average or high, regression models seem to highlight other variables as more predictive than intelligence. In our case, as Study 1 and 2 mentioned as an important future line of research enquiry, visual and verbal WM had an important effect on Faux Pas test (visual, verbal and mixed).

\subsubsection{Working Memory influence (Study 3)}

As explained in more detail in the faux pas explanation of two models of perspectives, it is easy to see how a faux pas is sensitive to individual differences in two Executive Functions. The first would be inhibition, because in order to understand a faux pas it is necessary to inhibit one's own perspective or reality. The other, would be the WM, as one needs to update the relevant information in WM for each new scenario (Li et al., 2013).

Following this line, in Study 3, we attempted to control the burden on visual and verbal WM, given how demanding a faux pas scenario can be, by placing the images (visual and mixed modality) or using some auditory reminders (verbal modality) while the questions were answered by the participants. Autistic and NT children participated in this third study.

One of the most striking results of Study 3 is the correlation and prediction displayed by WM in all modalities of the Faux Pas test, specifically, solely in the group of autistic children. The prediction of WM was approximately the same in all the faux pas scenarios: visual and verbal WM explained $19 \%$ to $28 \%$ of variability in faux pas test outcomes (verbal, visual, and mixed).

In addition, the correlation between WM and Faux Pas results was coherent when modalities were analysed (e.g., visual WM correlated with visual Faux Pas results). Verbal WM correlated to verbal, visual and mixed Faux Pas. This may be explained by the fact that in all the modalities there was at least some type of verbal information (i.e., in the visual modality, verbal information appeared when the questions were asked). Visual WM correlated only with visual and mixed Faux Pas. In this case, no significant correlation was found between visual WM and verbal Faux Pas, due to any visual information being presented in verbal Faux Pas. 
On the other hand, although there were no differences in WM between NT and ASD groups, in the autistic group the WM caused an effect in their results of the Faux Pas test, whereas in the NT group WM did not correlate with any modality of Faux Pas. Therefore, WM was only correlated and explained the variability in Faux Pas test in the autistic group.

One of the reasons that could explain these results was contemplated in Study 3. Autistic individuals may process information and abstractly assign meaning differently, which is consistent - again - with the WCC. Following this theory, if autistic individuals attend to small elements, it is more like that the memory load may be higher, in order to remember the complete story. Also, this process may happen in parroting, which could occur during the mental process of listening to the story and the corresponding questions, mentally repeating a particular fragment, and entering into a loop. Parroting effect also happened in Study 2, when children repeated the last sentence that the narrator or character said, or even the question that was asked to them. Therefore, this factor would be another element to control, as it appears in the germinal study of Baron-Cohen and team (1999).

How autistic people process information may influence their understanding, and therefore, their responses.

\subsubsection{Modality of presentation influence on complex mentalistic reasoning (Study}

\section{3)}

Study 3 contemplated three modalities: visual, verbal and mixed modality of faux pas stories. Autistic children's comprehension was better in the mixed modality and significant differences were found in the ASD group between mixed and verbal modality.

Thereby, the third study investigated how complex is verbal mentalistic reasoning for autistic individuals, through purely verbal ToM stories. Indeed, this difficulty seemed to decrease when visual stimuli are added to the verbal information in advanced ToM task. This mix (visual and verbal stimuli) may benefit autistic children, because, as commented in the objective 1 (Study 1) the level of abstraction is lower as the level of imagination is lower as well. Again, verbal information difficulties found in autistic children could be explained by the high imaginative and abstraction demands of deducing what is happening in a purely verbal scenario, as happens in everyday life in a telephone conversation scenario. In this sense, the presence of images in this study could reduce the imagination loads of the task.

As commented in the objective 1, visual imagery is inherently concrete, depicting specific things. For example, the word kitchen refers to many types of furniture, tools or electronic 
appliances. Nevertheless, an image of a kitchen will represent a specific kitchen, with a specific table, fridge, oven, forks, knives and spoons. In the same way, when someone visualises an accidental situation (i.e., in vignettes), some elements employed to achieve the understanding may be depicted. For example, in the case of the Cook story (Appendix 3, Figure 7.8), images can help to visualise that the speaker was not present when the important information was said. Also, images can help to better understand the role of the three characters of the story. Importantly - and regarding to ToM competence - visual representations, as compared to verbal representations, are more emotionally salient. Therefore, visual scenarios (vignettes, illustrations, cartoons) can facilitate emotional responses, and in the same way, it could facilitate the link between reasoning to mental states and empathy.

Moreover, as commented in 5.1.2.2 section, these difficulties when processing verbal information could be supported by WCC theory. Following this proposal, autistic children could have understood the verbal fragments of the text (per separate, locally), but they may not be able to make sense of an overall story (a whole story, globally). In other words, they could recognise the parts without comprehending the big picture.

On the other hand, as commented in Study 3, autistic people could have the so-called 'ASD advantage' on visual tasks (Blaser et al., 2014, terminology). As in Blocks subtest it has been well-documented that happens, autistic individuals can show extraordinary performance in some visual abilities. This capacity is also shown in Embedded Figures (Almeida et al., 2014) or Visual search (Joseph et al., 2017). However, this 'advantage' could be explained also based on WCC theory and the way that autistic individuals process the information.

To summarise, the empirical evidences of Study 3, can provide valuable clues for practical implication for autistic individuals. As commented at the beginning of this second aim, the finding that mixed modality may benefit autistic children is considered the most useful in terms of intervention or construction of ToM tasks.

Regarding the implications of the mixed modality for autistic children, some points could be highlighted in order to understand more concretely how visual images can help in a verbal faux pas scenario.

Firstly, mental states are not tangible in a purely verbal scenario. However, if the scenario is verbal and visual, autistic children may understand the scenario better through the images, concretely if the face of the characters is illustrated: face gestures or emotions.

Second, some details can be more salient when the scenario is mixed. For example, if it exists an important object exists it will be highlighted and remembered if it is drawn in the vignettes. 
Third, the number of characters in a story is less abstract if the characters are drawn and identified in the vignettes. Contrary, if the information is only verbal or auditory, it can be confusing who is talking or how many people there are in the story.

Finally, it is also helpful for autistic individuals that all the important information (both visual and verbal) is given at once. A faux pas has a high verbal weight, however if the images are presented at the same time of the verbal information, visual information enables the participants to follow the stories and improve their comprehension.

\subsubsection{Analyse the differences between Moral Transgressions and Faux Pas tasks for both autistic and NT participants (Study 4).}

The agent's morality understanding is complicated due to the mental states involved for construing a correct judgement. The mental states attributed to the character (intention) often do not coincide with the final outcome. Autistic people have been shown to predominantly committed errors when they judge a person because they used to base their judgement on the outcomes, more than the intentions (Abell et al., 2000; Baron-Cohen et al., 1985, 2000; Bowler, 1992; Castelli et al., 2002; Margoni \& Surian, 2016; Surian \& Leslie, 1999).

In Study 4, only mixed tasks were used, due to the ASD group better understanding these tasks in Study 3. The tasks contemplated were designed to test participants in cases of unambiguous valences (Moral Transgressions task) and ambiguous valences (Faux Pas test).

The main findings of Study 4 revealed that autistic and NT children were able to performed similarly in both Moral Transgression and Faux Pas task, and they obtained very similar scores in the judgements of intention, agent, action and outcome. Therefore, autistic children were capable of distinguishing between deliberate and accidental intentionality, which would be the main distinction for making appropriate judgements of an agent (as previous studies; e.g., Grant et al., 2005).

However, subtle differences were found between groups. When Moral Transgressions stories were more related to harming the victim directly (versus impersonal damage, such as stealing an object; in Study 4 it was called object as a mediator), stories were considered more complex, and significant differences were found between ASD and NT groups. This personal harming implies understanding mental states of the two characters: the perpetrator and the victim. Therefore, regardless, the scenarios presented are based on ambiguous or unambiguous valences, to resolve them properly, all the mental states information has to be understood. 
An important part of Study 4 was focused on judging the intention and morality of the agent, act and outcome. Although autistic children understood the intention, they judged the agent as a 'bad person' even when the intention was understood as accidental. As commented in Buon et al. (2013), Moran et al. (2011) and Zalla et al., 2009, autistic children rated the agent of an accidental transgression as more malicious or with intention. It was also observed that non-significant differences were found between the morality of the agent in Moral Transgressions and Faux Pas test in the autistic group. Namely, autistic children judged in the same way the agent that causes the damage in the same way when his / her act was deliberate or accidental. Contrary, in the NT group, these significant differences did exist: the agent of Moral Transgression stories were judged more severely than in the Faux Pas stories.

Thereby, the agent in Faux Pas test was judged as bad in Faux Pas stories by autistic children. This judgement, although is quite subtle, could be helpful in understanding these minimum differences in the complex mentalistic information processing of autistic individuals. Some explanations can be analysed from this finding:

a) ToM impairments are affecting the judgements: 'the agent is bad because the outcome is bad'.

Following this affirmation, as studied by Margoni \& Surian (2016), autistic children would seem to have developed the understanding of moral judgments - as unambiguous cases - although, possibly this would be masked by the reduced presence of mentalistic aspects in this type of tasks, for their preferential attention to external variables that are not conclusive (such as crying), or for the non-ambiguity in the valences of intention and results of the Moral Transgression stories.

\section{b) The difficulty to inhibit the information from one path (or perspective) and integrate the information from the other path.}

Following this affirmation, autistic individuals could have some problems inhibiting the information of the path that they know as observers, and integrating the information of the other path, based on speaker's knowledge / belief. Thereby, autistic children in this study could fail in these perspective's changes.

Therefore, although it was expected some significant differences between groups, NT and autistic children reasoned similar. Subtle differences were related to the difficulties of autistic individuals in advanced ToM tasks and in their inflexibility for inhibiting some thoughts and consequently, misinterpreting the false beliefs or the intentions of the agents. 


\subsection{General Discussions and Conclusions Summary}

In summary, there are some important findings in this thesis which could contribute to improving the understanding of the comprehension of the ambiguous and unambiguous cases based on ToM capacity in autistic children. This importance lies on the future implications that this research may have in the autistic, professional and scientific community. Key findings from this research are highlighted below:

a) The importance of visual information when advanced ToM tasks are presented.

Scenarios of purely verbal information when presenting a complex mental situation seem to be difficult. In fact, verbal information is necessary in stories where an important part is based on dialogues, prosody and narration. However, if at this verbal information is added some visual aids (i.e., images or vignettes), Study 3 corroborated that the understanding increases (as corroborated by Study 3). As detailed in 5.1.2.3 Modality of presentation influence on complex mentalistic reasoning, the presence of images when the stories are read and even when the questions are answered can reduce the imagination loads of the task.

Visual information is necessary for autistic individuals, as they can focus on more relevant details for the story comprehension, such as the face of the characters, emotions, important elements or the identification of the characters. A mix of both verbal and visual information facilitates the participants to identify the relevant elements of the story for its better comprehension.

b) The importance of controlling the burden on WM in advanced ToM tasks.

As scenarios in advanced ToM tasks are WM demanding, WM should be controlled for in some way. The influence of WM in the Faux Pas test in autistic participants only is detailed in Study 3 and in the section 5.1.2.2 Working Memory influence. WM was controlled by using the images and auditory reminders while the questions were being answered by the participants. Despite this, regardless of the modality and how the WM was controlled (i.e., images or verbal reminders), WM explained the variability in Faux Pas responses, solely in the ASD group. These findings may be related to the different information processing of autistic individuals, who may attend to small elements rather than comprehend the bigger picture (WCC). WCC theory supports the hypothesis that 
WM loads may be greater for autistic people and such as, they may fail to remember the complete story, attending only to some sentences or details. It should be noted that better measures of WM controlling should be considered, as being WM an important factor for autistic individuals that may affect comprehension of advanced ToM tasks. (This will be addressed further in the 5.3 Limitations and Implications section).

c) The importance of mental states when reasoning about morality.

Mental states generate physical events. Theses physical events can generate mental states when situations are judged or evaluated. In this way, from mental states to mental reasoning, some information can be lost, mental states misinterpreted or intention inappropriately distinguished. Further, autistic children may judge some situations based on everyday life, such as the tasks presented (specifically in Faux Pas), assigning more blame to the agent-speaker of the faux pas. In the open and forced-choice questions of different studies of this thesis, autistic children judged the agent of the accidental action as more malicious or with intention. Autistic children appear to focus more on the bad outcome and less on the lack of negative intent. However, autistic children seemed to recognise the intention when it was compared between ambiguous and unambiguous cases in Study 4. Possibly, the difficulty to process the mentalistic information (i.e., false belief, lack of awareness, intention) as well as the difficulty to inhibit the knowledge from two perspectives, are sufficient reasons to explain the complexity of a moral transgression and a faux pas for an autistic individual. 


\subsection{Limitations and Implications}

\subsubsection{Limitations}

Along with the main findings of this study, it is also important to consider the limitations in the design of the tasks. This can also help to construct of a formal conclusion for explaining the subtle differences found between groups.

Firstly, in relation to the Theoretical Framework, the work presented here adopted a grounded theory approach as well as empirical results of children with and without autism. As a result, some models were proposed in the Introduction section and Studies aimed to explain how our mind can interpret others' behaviors from different perspectives, theoretically. The models based on mentalistic aspects of this work were in some cases re-created from previous studies. To contemplate the ToM in these models can make the interpretation and understanding of how we process everyday information more complex. However, complexity is inevitable when we try to understand how our mind works.

Secondly, regarding the sample size used, 62 children took part in the majority of studies. As the first contact of the children and their families were through previous advice of professionals and teachers, at least, 20 children who took part in the study did not fulfill the established criteria. Some participants were not included for further examination due to their IQ $(<80)$ or because they failed the first-order FB task. Some of those disqualified autistic participants were included in a restructured group in Study 2 (IQ range from 70 to 85), which was not originally planned for.

A report of all the participants included and excluded from the studies was written for their families and school, including their scores, implications and possible intervention. This delayed all the work and research for a few months.

Although at first glance, the final sample is average size, generalisation of the findings require careful interpretation, especially when we refer to some conditions, such as 'autistic individuals', a highly heterogeneous group.

Thirdly, in relation to the materials used and more concretely in morality, more moral situations could have been analysed in the present thesis. For example, failed or attempted cases, moral dilemmas as personal and impersonal, or moral scenarios with different severity: damage a property / harm a person. Following the structure of Figure 2-3, deliberate, accidental, 
failed and benign situations could be studied for knowing the relation, differences and difficulties between these cases of different intentions, control of the action and outcomes. In the same way, due to the novelty of Moral Transgression and Faux Pas tasks created and adapted by the research group, it should be contemplated other advanced ToM tasks. A good choice would have been the well-known Strange Stories (Happé, 1994a), for comparing these classical stories to the results of the novel scenarios. In total, a limited number of stories were used and more everyday situations usual for children could have been created, as the stories based on everyday life are scarce.

In this sense, more modalities of presentation could be considered, such as a video-tape format, which seems closer to real-life situations. Gestures, glances and body language could have been contemplated in the above-mentioned modality.

Fourthly, related to the variables analysed, several questions were asked (forced-choice and open responses). However, other variables could be contemplated with a different format. In relation to moral aspects, variables such as mixed emotions, culpability, punishment or alternative acts could be interesting to further investigate to understand more about the judgements that children can make. The same questions could be analysed in accidental or failed situations, due to the interest in discovering differences when the cases are ambiguous. Also, different ranks could be tested, for example, it could have been interesting to collect responses using multiple choice questions or scaled questions (Likert scale).

Finally, regarding the results, further study is required to clarify some relationships and influences were mentioned in this work. In particular, WM showed a relevant prediction for autistic children in all the modalities (with a similar $\mathrm{R}^{2}$ ), although the WM load was different in each modality. In the Visual Faux Pas stories, the WM was more controlled, due to the images being shown while the child answered the questions. In the Verbal Faux Pas stories, verbal information was not placed in front of the participant, only some auditory reminders were used in the questions. This calls for further research. One possible future analysis could be to consider another modality which includes all the information on one screen: vignettes, text, auditory narration, dialogs and questions. In this modality, WM would be better controlled. A future study could also analyse verbal modality with auditory stimuli without reminders, to a complete-mixed modality with all the information presented at once. 


\subsubsection{Implications}

This study provides practical implications for autistic children and adolescents, and for the professionals and families that work with them. Firstly, results indicated how beneficial the visual information can be for comprehending affective aspects and important details (the faces of the characters, emotions, relevant elements...), when verbal stories are presented: a mix between images and narration or dialogs can facilitate the participants in identifying the important elements of the scenario. Similarly, the presence of images when verbal information is read or heard can reduce the imagination loads of the task and facilitate participants reasoning. Secondly, findings encourage researchers and professionals to design future tools and materials that contemplate WM and attentional controlling, with some reminders, to enable the participants to better follow the stories properly. As explained, a task which includes all the information on one screen / paper can be beneficial for autistic children by having all the information available at the same time, and the WM load would be better controlled. Thirdly, the figures used in the Theoretical Framework to break deliberate and accidental situations into aspects play an important role in the comprehension in terms of ToM, but also for creating new activities or stories based on this empirical approach. Fourthly, these studies have demonstrated that results were similar when we compared autistic and NT children. However, the subtle differences found can shed some light for paving the way to understand how autistic individuals process the information. Results should not be understood as 'pass' or 'fail', the focus should be on the need to strengthen those aspects that autistic people have advantage and clarify how to compensate when they experience difficulty. Finally, in order to apply the finding to real life, we simply need to observe a school playground during a break to detect interpersonal relationships, and to see that one of the major sources of conflict is a mistaken interpretation of intention. Now, let's imagine that in this scenario there are autistic children, who may have problems with understanding others' intentions. If, on the one hand, autistic individuals misinterpret the other child's good intention as negative, they will probably lose opportunities to make friends or be unable to keep friends. Conversely, misinterpreting a negative intention as a good one could be dangerous for them, and make them vulnerable to bullying. For this social reason, the present doctoral thesis tried to focus on these difficulties - often subtle difficulties and which go unnoticed in social contexts - that autistic people usually encounter in their daily lives, associated with intention and morality. Knowing in what aspects autistic individuals experience difficulty would shed some light on finding out how to guide intervention for having a positive impact on their quality of life. In fact, improving experiences for autistic individuals through better design, can be beneficial for everyone. 
Capítulo 6 Referencias / Chapter 6 References 
Abell, F., Happe, F., \& Frith, U. (2000). Do triangles play tricks? Attribution of mental states to animated shapes in normal and abnormal development. Cognitive Development, 15(1), 1-16.

Alicke, M. D. (2000). Culpable control and the psychology of blame. Psychological bulletin, 126(4), 556. doi: 10.1037/0033-2909.126.4.556

Almeida, R. A., Dickinson, J. E., Maybery, M. T., Badcock, J. C., \& Badcock, D. R. (2010). Visual search performance in the autism spectrum II: The radial frequency search task with additional segmentation cues. Neuropsychologia, 48, 4117-4124. doi: 10.1016/j.neuropsychologia.2010.10.009

American Psychiatric Association. (2013). DSM-5. American Psychiatric Publishing.

Amit, E., Algom, D., \& Trope, Y. (2009). Distance-dependent processing of pictures and words. Journal of Experimental Psychology: General, 138(3), 400. doi: $10.1037 / \mathrm{a} 0015835$

Amit, E., \& Greene, J. D. (2012). You see, the ends don't justify the means: Visual imagery and moral judgment. Psychological science, 23(8), 861-868. doi: $10.1177 / 0956797611434965$

Apperly, I. A., Samson, D., \& Humphreys, G. W. (2005). Domain-specificity and theory of mind: Evaluating neuropsychological evidence. Trends in Cognitive Sciences, 9, 573 577. doi: 10.1016/j.tics.2005.10.004

Apperly, I. A., Samson, D., Chiavarino, C., \& Humphreys, G. W. (2004). Frontal and temporo-parietal lobe contributions to theory of mind: Neuropsychological evidence from a false-belief task with reduced language and executive demands. Journal of Cognitive Neuroscience, 16, 1773-1784. doi: 10.1162/0898929042947928

Armsby, R. E. (1971). A reexamination of the development of moral judgments in children. Child development, 1241-1248. doi: 10.2307/1127807

Astington, J. W. (2001). The Future of Theory of Mind Research: Understanding Motivational States, the Role of Language, and Real-World Consequences. Child Development, 72(3), 685-687. doi: 10.1111/1467-8624.00305

Astington, J. W., Harris, P. L., \& Olson, D. R. (Eds.). (1988). Developing Theories of Mind. Cambridge: Cambridge University Press. 
Autism-Europe (2018). Prevalence rate of autism. Brussels, Belgium: Autism-Europe. Retrieved from: http://www.autismeurope.org/about-autism/prevalence-rate-ofautism/

Baddeley, A. (1992). Working memory. Science, 255(5044), 556-559. doi: 10.1126/science. 1736359

Baird, J. A., \& Astington, J. W. (2004). The role of mental state understanding in the development of moral cognition and moral action. New Directions for Child and Adolescent Development, 2(103), 37-49. doi: 10.1002/cd.96

Baker, C. L., Saxe, R., \& Tenenbaum, J. B. (2009). Action understanding as inverse planning. Cognition, 113(3), 329-349.

Banerjee, R., \& Watling, D. (2005). Children's understanding of faux pas. Hellenic Journal of Psychology, 2, 27-45.

Banerjee, R., Watling, D., \& Caputi, M. (2011). Peer relations and the understanding of faux pas: Longitudinal evidence for bidirectional associations. Child development, 82(6), 1887-1905. doi: 10.1111/j.1467-8624.2011.01669.x

Baron-Cohen, S. (1995). The eye direction detector (EDD) and the shared attention mechanism (SAM): Two cases for evolutionary psychology. In Portions of this paper were presented at the Society for Research in Child Development Conference, New Orleans, Mar 1993; the British Psychological Society, Welsh Branch, "Faces" Conference, U Wales Coll of Cardiff, Sep 1993; and the British Society for the Philosophy of Science" Roots of Joint Reference" Conference, U Bristol, Nov $1993 .$. Lawrence Erlbaum Associates, Inc.

Baron-Cohen, S. (1997). How to build a baby that can read minds: Cognitive mechanisms in mindreading. The maladapted mind: Classic readings in evolutionary psychopathology, 207-239.

Baron-Cohen, S., Jolliffe, T., Mortimore, C., \& Robertson, M. (1997). Another advanced test of theory of mind: Evidence from very high functioning adults with autism or Asperger Syndrome. Journal of Child Psychology and Psychiatry, 38(7), 813-822. doi: 10.1111/j.1469-7610.1997.tb01599.x

Baron-Cohen, S., Leslie, A. M., \& Frith, U. (1985). Does the autistic child have a "theory of mind"? Cognition, 21, 37-46. doi: 10.1016/0010-0277(85)90022-8 
Baron-Cohen, S., O'Riordan, M., Stone, V., Jones, R., \& Plaisted, K. (1999). Recognition of faux pas by normally developing children and children with Asperger syndrome or high-functioning autism. Journal of Autism and Developmental Disorders 29(5), 407418.

Bishop, D. V. M., \& Norbury, C. F. (2002). Exploring the borderlands of autistic disorder and specific language impairment: A study using standardized diagnostic instruments. Journal of Child Psychology and Psychiatry, 43(7), 917-929. doi: 10.1111/14697610.00114

Blair, R. J. R. (1996). Brief report: Morality in the autistic child. Journal of autism and developmental disorders, 26(5), 571-579.

Blaser, E., Eglington, L., Carter, A. S., \& Kaldy, Z. (2014). Pupillometry reveals a mechanism for the autism spectrum disorder (ASD) advantage in visual tasks. Scientific Reports, 4, 4301. doi: 10.1038/srep04301

Bowler, D. M. (1992). “Theory of Mind” in Asperger's Syndrome. Journal of Child Psychology and Psychiatry, 33(5), 877-893.

Bretherton, I. (1991). Intentional communication and the development of an understanding of mind. Children's theories of mind: Mental states and social understanding, 49-75.

Brewer, R., Marsh, A., Catmur, C., Cardinale, E. M., Stoycos, S., Cook, R., \& Bird, G. (2015). The impact of autism spectrum disorder and alexithymia on judgments of moral acceptability. Journal of Abnormal Psychology, 124(3), 589-95. doi: 10.1037/abn0000076

Buon, M., Dupoux, E., Jacob, P., Chaste, P., Leboyer, M., \& Zalla, T. (2013). The role of causal and intentional judgments in moral reasoning in individuals with high functioning autism. Journal of autism and developmental disorders, 43(2), 458-470. doi: $10.1007 / \mathrm{s} 10803-012-1588-7$

Callaghan, T., Rochat, P., Lillard, A., Claux, M. L., Odden, H., Itakura, S., \& Singh, S. (2005). Synchrony in the onset of mental-state reasoning evidence from five cultures. Psychological Science, 16(5), 378-384. doi: 10.1111/j.09567976.2005.01544.x

Castelli, F., Frith, C., Happé, F., \& Frith, U. (2002). Autism, Asperger syndrome and brain mechanisms for the attribution of mental states to animated shapes. Brain, 125(8), 1839-1849. doi: 10.1093/brain/awf189 
Centro para el Control y la Prevención de Enfermedades (2018). Prevalencia del autismo levemente más alta según informe de la Red ADDM de los CDC. GA, USA: $C D C$. Retrieved from https://www.cdc.gov/spanish/mediosdecomunicacion/comunicados/p_prevalenciaautismo_042618.html

Chakroff, A., \& Young, L. (2015). How the mind matters for morality. AJOB Neuroscience, 6(3), 43-48.

Chandler, M. J., Sokol, B. W., \& Hallett, D. (2001). Moral responsibility and the interpretive turn: Children's changing conceptions of truth and rightness. Intentions and intentionality: Foundations of social cognition, 345-365.

Charman, T., Pickles, A., Simonoff, E., Chandler, S., Loucas, T. \& Baird, G. (2011) IQ in children with autism spectrum disorders: data from the Special Needs and Autism Project (SNAP). Psychological Medicine: A Journal of Research in Psychiatry and the Allied Sciences 41(3): 619-627. doi: 10.1017/S0033291710000991

Cohen, M. J., \& Sloan, D. L. (2007). Visual supports for people with autism: A guide for parents and professionals. Bethesda, MD: Woodbine House.

Costanzo, P. R., Coie, J. D., Grumet, J. F., \& Farnill, D. (1973). A reexamination of the effects of intent and consequence on children's moral judgments. Child Development, 154-161.

Cushman, F. (2015). Deconstructing intent to reconstruct morality. Current Opinion in Psychology, 6, 97-103. doi: 10.1016/j.copsyc.2015.06.003

Cushman, F., Dreber, A., Wang, Y., \& Costa, J. (2009). Accidental outcomes guide punishment in a "trembling hand" game. PloS one, 4(8), e6699. doi: 10.1371/journal.pone.0006699.

Cushman, F., Sheketoff, R., Wharton, S., \& Carey, S. (2013). The development of intentbased moral judgment. Cognition, 127(1), 6-21. doi: 10.1016/j.cognition.2012.11.008.

De Villiers, P.A., \& De Villiers, J.G. (2012). Deception dissociates from false belief reasoning in deaf children: Implications for the implicit versus explicit theory of mind distinction. British Journal of Developmental Psychology, 30(1), 188-209. doi: 10.1111/j.2044-835X.2011.02072.x 
DeScioli, P., Asao, K., \& Kurzban, R. (2012). Omissions and byproducts across moral domains. PloS one, 7(10), e46963. doi: 10.1371/journal.pone.0046963

Dettmer, S., Simpson, R. L., Myles, B. S., \& Ganz, J. B. (2000). The use of visual supports to facilitate transitions of students with autism. Focus on Autism and Other Developmental Disabilities, 15(3), 163-169. doi: 10.1177/108835760001500307

Duncan, A. W., \& Bishop, S. L. (2015). Understanding the gap between cognitive abilities and daily living skills in adolescents with autism spectrum disorders with average intelligence. Autism, 19(1), 64-72. doi: 10.1177/1362361313510068

Durrleman, S., \& Franck, J. (2015). Exploring links between language and cognition in autism spectrum disorders: Complement sentences, false belief, and executive functioning. Journal of Communication Disorders, 54, 15-31. doi:10.1016/j.jcomdis.2014.12.001

Eaves, L. C., \& Ho, H. H. (1997). School placement and academic achievement in children with autistic spectrum disorders. Journal of Developmental and Physical Disabilities, 9(4), 277-291.

Eddy, C. M., Mitchell, I. J., Beck, S. R., Cavanna, A. E., \& Rickards, H. E. (2010). Impaired comprehension of nonliteral language in Tourette syndrome. Cognitive and Behavioral Neurology, 23(3), 178-184. doi: 10.1097/WNN.0b013e3181e61cb7

Elkind, D., \& Dabek, R. F. (1977). Personal injury and property damage in the moral judgments of children. Child Development, 518-522. doi: 10.2307/1128648

Farmer, C. (2013). Leiter International Performance Scale-Revised (Leiter-R). In F. R. Volkmar, Encyclopedia of autism spectrum disorders, 1732-1735. New York: Springer.

Fisher, N., Happé, F. \& Dunn, J. (2005). The relationship between vocabulary, grammar and false belief task performance in children with Autistic Spectrum Disorders and children with moderate learning difficulties. Journal of Child Psychology and Psychiatry, 46 (4), 409-419. doi: 10.1111/j.1469-7610.2004.00371.x

Frith, C., \& Frith, U. (2005). Theory of mind. Current Biology, 15(17), R644-R645. doi: 10.1016/j.cub.2005.08.041

Frith, U. (1989). Autism: Explaining the enigma. Oxford: Basil Blackwell. 
Garcia-Molina, I. (en prensa/in press). Preferencias terminológicas acerca del autismo según participantes de un MOOC sobre inclusión educativa. Psicología, Conocimiento y Sociedad.

Garcia-Molina, I., Clemente Estevan, R. A., \& Andrés-Roqueta, C. (2014). Intervenció en Teoria de la Ment d'Alt Nivell. Més enllà de "Sally i Anne" Anàlisi d'un cas. Fòrum de Recerca (19), 481-489.

Garcia-Molina, I., Clemente-Estevan, R. A., Andrés-Roqueta, C., \& Rodríguez, L. (2016). Intervención mediante historias complejas de teoría de la mente. Meteduras de pata, juicios morales e ironías [Intervention using advanced stories of theory of mind. Faux pas, moral judgments, and ironies]. Castellón, Spain: Universitat Jaume I, Psique. doi: 10.6035/Psique.2016.17

Girard, D. \& Garcia-Molina, I. (2018). Theory of Mind and intelligence. The importance of visual stimulus in autism. INSAR Annual Meeting, Rotterdam, Netherlands.

Goldman, A. I. (2012). Theory of mind. The Oxford handbook of philosophy of cognitive science, 402-424. doi: 10.1093/acprof:osob1/9780199874187.003.0002

González-Gadea, M. L., Baez, S., Torralva, T., Castellanos, F. X., Rattazzi, A., Bein, V., Rogg, K., Manes, F. \& Ibanez, A. (2013). Cognitive variability in adults with ADHD and AS: Disentangling the roles of executive functions and social cognition. Research in Developmental Disabilities, 34, 817-830. doi: 10.1016/j.ridd.2012.11.009

Gopnik, A., \& Astington, J. W. (1988). Children's understanding of representational change and its relation to the understanding of false belief and the appearance-reality distinction. Child development, 26-37.

Grant, C. M., Boucher, J., Riggs, K. J., \& Grayson, A. (2005). Moral understanding in children with autism. Autism, 9(3), 317-331. doi: 10.1177/136236130505541

Greene, J. D., Sommerville, R. B., Nystrom, L. E., Darley, J. M., \& Cohen, J. D. (2001). An fMRI investigation of emotional engagement in moral judgment. Science, 293(5537), 2105-2108. doi: 10.1126/science. 1062872

Greene, J. D., Nystrom, L. E., Engell, A. D., Darley, J. M., \& Cohen, J. D. (2004). The neural bases of cognitive conflict and control in moral judgment. Neuron, 44(2), 389-400. doi: 10.1016/j.neuron.2004.09.027 
Gregory, C., Lough, S., Stone, V., Erzinclioglu, S., Martin, L., Baron-Cohen, S., \& Hodges, J. R. (2002). Theory of mind in patients with frontal variant frontotemporal dementia and Alzheimer's disease: Theoretical and practical implications. Brain, 125(4), 752764.

Grundy, D. (2011). Friend or fake? Mate crimes and people with learning disabilities. Journal of Learning Disabilities and Offending Behaviour, 2(4), 167-169.

Guinea, A. Tirapu-Ustárroz, J. \& Pollán, M. (2007). Teoría de la mente en la esquizofrenia. Análisis y Modificación de Conducta, 33(148).

Haidt, J. (2007). The new synthesis in moral psychology. Science, 316(5827), 998-1002. doi: $10.1126 /$ science. 1137651

Happé, F. G. (1994a). An advanced test of theory of mind: Understanding of story characters' thoughts and feelings by able autistic, mentally handicapped, and normal children and adults. Journal of Autism and Developmental Disorders, 24(2), 129-154. doi: 10.1007/BF02172093

Happé, F. G. (1994b). Wechsler IQ profile and theory of mind in autism: A research note. Journal of Child Psychology and Psychiatry, 35(8), 1461-1471. doi: 10.1111/j.1469-7610.1994.tb01287.x

Happé, F. G. (1995). The role of age and verbal ability in the theory of mind task performance of subjects with autism. Child development, 66(3), 843-855. doi: $10.2307 / 1131954$

Happé, F., \& Frith, U. (2006). The weak coherence account: Detail-focused cognitive style in autism spectrum disorders. Journal of Autism and Developmental Disorders, 36(1), 525. doi: 10.1007/s10803-005-0039-0

Hartshorne, H., \& May, M. A. (1930). A summary of the work of the character education inquiry. Religious Education, 25(7), 607-619. doi: 10.1080/0034408300250702

Hauser, M. (2006). Moral minds: How nature designed our universal sense of right and wrong. Ecco/HarperCollins Publishers.

Hayes, G. R., Hirano, S., Marcu, G., Monibi, M., Nguyen, D. H., \& Yeganyan, M. (2010). Interactive visual supports for children with autism. Personal and Ubiquitous Computing, 14(7), 663-680. doi: 10.1007/s00779-010-0294-8 
Heider, F. (1958). The psychology of interpersonal relation. New York: Wiley. doi: $10.1037 / 10628-000$

Helwig, C. C., Hildebrandt, C., \& Turiel, E. (1995). Children's judgments about psychological harm in social context. Child development, 66(6), 1680-1693.

Helwig, C. C., Zelazo, P. D., \& Wilson, M. (2001). Children's judgments of psychological harm in normal and noncanonical situations. Child development, 72(1), 66-81.

Hughes, C., \& Leekam, S. (2004). What are the links between theory of mind and social relations? Review, reflections and new directions for studies of typical and atypical development. Social development, 13(4), 590-619.

Imamoğlu, E. O. (1975). Children's awareness and usage of intention cues. Child Development, 39-45.

Inbar, Y., Pizarro, D. A., \& Cushman, F. (2012). Benefiting from misfortune: When harmless actions are judged to be morally blameworthy. Personality and Social Psychology Bulletin, 38(1), 52-62.

Jarrold, C., Gilchrist, I. D., \& Bender, A. (2005). Embedded figures detection in autism and typical development: Preliminary evidence of a double dissociation in relationships with visual search. Developmental Science, 8(4), 344-351. doi: 10.1111/j.14677687.2005.00422.x

Johnston, S., Nelson, C., Evans, J., \& Palazolo, K. (2003). The use of visual supports in teaching young children with autism spectrum disorder to initiate interactions. Augmentative and Alternative Communication, 19(2), 86-103. doi: 10.1080/0743461031000112016

Joseph, R. M., Keehn, B., Connolly, C., Wolfe, J. M., \& Horowitz, T. S. (2009). Why is visual search superior in autism spectrum disorder? Developmental Science, 12, 10831096. doi: 10.1111/j.1467-7687.2009.00855.x

Juhos, C., Quelhas, A. C., \& Byrne, R. M. (2015). Reasoning about intentions: Counterexamples to reasons for actions. Journal of Experimental Psychology: Learning, Memory, and Cognition, 41(1), 55.

Kahane, G. (24 de junio, 2010). A Universal Moral Code? [Practical Ethics]. Recuperado de http://blog.practicalethics.ox.ac.uk/2010/06/a-universal-moral-code/_(acceso 21 de marzo). 
Kenny, L., Hattersley, C., Molins, B., Buckley, C., Povey, C., \& Pellicano, E. (2016). Which terms should be used to describe autism? Perspectives from the UK autism community. Autism, 20(4), 442-462. doi: 10.1177/1362361315588200

Kiley Hamlin, J., Ullman, T., Tenenbaum, J., Goodman, N., \& Baker, C. (2013). The mentalistic basis of core social cognition: Experiments in preverbal infants and a computational model. Developmental science, 16(2), 209-226. doi:

$10.1111 /$ desc. 12017

Killen, M., Mulvey, K. L., Richardson, C., Jampol, N., \& Woodward, A. (2011). The accidental transgressor: Morally-relevant theory of mind. Cognition, 119(2), 197-215. doi: 10.1016/j.cognition.2011.01.006

Kleinman, J., Marciano, P. L., \& Ault, R. L. (2001). Advanced theory of mind in highfunctioning adults with autism. Journal of Autism and Developmental Disorders, 31, 29-36.

Kline, P. (1991). Intelligence: The psychometric view. New York, NY: Routledge.

Knobe, J. (2005). Theory of mind and moral cognition: Exploring the connections. Trends in Cognitive Sciences, 9, 357-359. doi: 10.1016/j.tics.2005.06.011

Koster-Hale, J., Saxe, R., Dungan, J., \& Young, L. L. (2013). Decoding moral judgments from neural representations of intentions. Proceedings of the National Academy of Sciences, 201207992. doi: 10.1073/pnas. 1207992110

Lagnado, D. A., \& Channon, S. (2008). Judgments of cause and blame: The effects of intentionality and foreseeability. Cognition, 108(3), 754-770. doi:

10.1016/j.cognition.2008.06.009

Leslie, A. M., Knobe, J., \& Cohen, A. (2006). Acting intentionally and the side-effect effect: Theory of mind and moral judgment. Psychological science, 17(5), 421-427. doi: $10.1111 / \mathrm{j} .1467-9280.2006 .01722$

Leslie, A. M., Mallon, R., \& DiCorcia, J. A. (2006). Transgressors, victims, and cry babies: Is basic moral judgment spared in autism?. Social neuroscience, 1(3-4), 270-283. doi:10.1080/17470910600992197

Li, X., Wang, K., Wang, F., Tao, Q., Xie, Y., \& Cheng, Q. (2013). Aging of theory of mind: The influence of educational level and cognitive processing. International Journal of Psychology, 48(4), 715-727. doi: 10.1080/00207594.2012.673724 
Liberman, N., \& Trope, Y. (2008). The psychology of transcending the here and now. Science, 322(5905), 1201-1205. doi: 10.1126/science.1161958

Lin, I. F., Shirama, A., Kato, N., \& Kashino, M. (2017). The singular nature of auditory and visual scene analysis in autism. Philosophical Transactions of the Royal Society of London, Series B, Biological Sciences, 372(1714). doi: 10.1098/rstb.2016.0115

Liu, D., Wellman, H. M., Tardif, T., \& Sabbagh, M. A. (2008). Theory of mind development in Chinese children: a meta-analysis of false-belief understanding across cultures and languages. Developmental psychology, 44(2), 523. doi: 10.1037/0012-1649.44.2.523

Lord, C., Rutter, M., DiLavore, P. C., \& Risi, S. (2008). Autism diagnostic observation schedule: ADOS manual. Los Angeles: Western Psychological Services.

Lord, C., Rutter, M., \& Le Couteur, A. (1994). Autism Diagnostic Interview-Revised: a revised version of a diagnostic interview for caregivers of individuals with possible pervasive developmental disorders. Journal of autism and developmental disorders, 24(5), 659-685. doi: 10.1007/BF02172145

Loveland, K. A., Pearson, D. A., Tunali-Kotoski, B., Ortegon, J., \& Gibbs, M. C. (2001). Judgments of social appropriateness by children and adolescents with autism. Journal of Autism and Developmental Disorders, 31(4), 367-376.

Malle, B. F. (1999). How people explain behavior: A new theoretical framework. Personality and social psychology review, 3(1), 23-48. doi: 10.1207/s15327957pspr0301_2

Malle, B. F. (2004). How the Mind Explains Behavior: Folk Explanations. Meaning and Social Interaction MIT Press, Cambridge.

Malle, B. F., Guglielmo, S., \& Monroe, A. E. (2014). A theory of blame. Psychological Inquiry, 25(2), 147-186.

Malle, B., \& Knobe, J. (1997). The folk concept of intentional action. Journal of Experimental Social Psychology 33: 101-121.

Maoz, H., Tsviban, L., Gvirts, H. Z., Shamay-Tsoory, S. G., Levkovitz, Y., Watemberg, N., \& Bloch, Y. (2014). Stimulants improve theory of mind in children with attention deficit/hyperactivity disorder. Journal of Psychopharmacology, 28(3), 212-219. doi: $10.1177 / 0269881113492030$ 
Margoni, F., \& Surian, L. (2016). Mental State Understanding and Moral Judgment in children with Autistic Spectrum Disorder. Frontiers in Psychology, 7, 1478. doi: doi.org/10.3389/fpsyg.2016.01478

Margoni, F., \& Surian, L. (2017). Children's intention-based moral judgments of helping agents. Cognitive Development, 41, 46-64. doi: 10.1016/j.cogdev.2016.12.001

Martin, J. W., \& Cushman, F. (2016). The adaptive logic of moral luck. The Blackwell companion to experimental philosophy, 190-202. doi: 10.1002/9781118661666.ch12

Mayes, S. D., \& Calhoun, S. L. (2003). Analysis of WISC-III, Stanford-Binet:IV, and academic achievement test scores in children with autism. Journal of Autism and Developmental Disorders, 33, 329-341. doi: 10.1023/A:1024462719081.

Mikhail, J. (2007). Universal moral grammar: Theory, evidence and the future. Trends in Cognitive Sciences, 11, 143-52. doi:10.1016/j.tics.2006.12.007

Mikhail, J. (2011). Elements of Moral Cognition: Rawls' Linguistic Analogy and the Cognitive Science of Moral and Legal Judgment. Cambridge University Press. doi: 10.1017/CBO9780511780578

Milligan, K., Astington, J. W., \& Dack, L. A. (2007). Language and theory of mind: metaanalysis of the relation between language ability and false-belief understanding. Child development, 78(2), 622-646. doi: 10.1111/j.1467-8624.2007.01018.x

Minshew, N. J., \& Goldstein, G. (1998). Autism as a disorder of complex information processing. Mental Retardation and Developmental Disabilities Research Reviews, $4(2), 129-136$.

Moore, A. B., Clark, B. A., \& Kane, M. J. (2008). Who shalt not kill? Individual differences in working memory capacity, executive control, and moral judgment. Psychological science, 19(6), 549-557. doi: 10.1111/j.1467-9280.2008.02122.x

Moran III, J. D., \& O'Brien, G. (1983). The development of intention-based moral judgments in three-and four-year-old children. The Journal of genetic psychology, 143(2), 175179. doi: 10.1080/00221325.1983.10533549

Moran, J. M., Young, L. L., Saxe, R., Lee, S. M., O'Young, D., Mavros, P. L., \& Gabrieli, J. D. (2011). Impaired theory of mind for moral judgment in high-functioning autism. Proceedings of the National Academy of Sciences, 108(7), 2688-2692. doi: 10.1073/pnas. 1011734108 
Nobes, G. Panagiotaki, G., \& Pawson, C. (2009). The influence of negligence, intention and outcome on children's moral judgments. Journal of Experimental Child Psychology, 104, 382-397. doi: 10.1016/j.jecp.2009.08.001

Nobes, G., Panagiotaki, G., \& Bartholomew, K. J. (2016). The influence of intention, outcome and question-wording on children's and adults' moral judgments. Cognition, 157, 190-204. doi: 10.1016/j.cognition.2016.08.019

Nucci, L. (1987). Synthesis of research on moral development. Educational Leadership, 44(5), 86-92.

Ozonoff, S. (1997). Components of executive function in autism and other disorders. In J. Russell (Ed.), Autism as an executive disorder (pp. 179-211). New York: Oxford University Press. doi: 10.1093/med:psych/9780198523499.003.0006

Ozonoff, S., Pennington, B. F., \& Rogers, S. J. (1991). Executive function deficits in highfunctioning autistic individuals: relationship to theory of mind. Journal of child Psychology and Psychiatry, 32(7), 1081-1105.

Patil, I., Melsbach, J., Hennig-Fast, K., \& Silani, G. (2016). Divergent roles of autistic and alexithymic traits in utilitarian moral judgments in adults with autism. Scientific Reports, 6, 23637. doi: 10.1038/srep23637

Pearson, R. A. M., \& Pillow, B. H. (2016). Children's and adults' understanding of faux pas and insults. Journal of Educational and Developmental Psychology, 6(2), 14. doi: 10.5539/jedp.v6n2p14

Pedreño, C., Pousa, E., Navarro, J. B., Pàmias, M., \& Obiols, J. E. (2017). Exploring the components of advanced theory of mind in autism spectrum disorder. Journal of Autism and Developmental Disorders, 47(8), 2401-2409. doi: 10.1007/s10803-0173156-7

Perner, J. (1999). Theory of mind. Developmental psychology: Achievements and prospects, 205-230.

Perner, J., Leekam, S.R., \& Wimmer (1987). Three-year-olds' difficulty with false belief: The case for a conceptual dèficit. British Journal of Developmental Psychology, 5(2), 125-137. doi: 10.1111/j.2044-835X.1987.tb01048.x

Pettit, D., \& Knobe, J. (2009). The pervasive impact of moral judgment. Mind \& Language, 24(5), 586-604. doi: 10.1111/j.1468-0017.2009.01375.x 
Piaget, J. (1932/1965). The moral judgment of the child. Trans. M. Gabain. New York: Free Press.

Pierce, K., Glad, K. S., \& Schreibman, L. (1997). Social perception in children with autism: An attentional deficit? Journal of Autism and Developmental Disorders, 27, 265-282.

Premack, D. (1990). The infant's theory of self-propelled objects. Cognition, 36(1), 1-16. doi: 10.1016/0010-0277(90)90051-K

Premack, D., \& Woodruff, G. (1978). Does the chimpanzee have a theory of mind? Behavioral and Brain Sciences, 1, 512-526. doi: 10.1017/S0140525X00076512

Rogé, B., \& Mullet, E. (2011). Blame and forgiveness judgements among children, adolescents and adults with autism. Autism, 15(6), 702-712. doi: $10.1177 / 1362361310394219$

Rogers, J., Viding, E., Blar, J., Frith, U., and Happé, F. (2006). Autism spectrum disorder and psychopathy: shared cognitive underpinnings or double hit? Psychol. Med. 36, 17891798. doi: $10.1017 / \mathrm{S} 0033291706008853$

Russell, J. (1997). How executive disorders can bring about an inadequate 'theory of mind'. In J. Russell (Ed.), Autism as an executive disorder (pp. 256-304). New York: Oxford University Press. doi: 10.1093/med:psych/9780198523499.003.0008

Rutter, M., Bailey, A., \& Lord, C. (2003). The social communication questionnaire: Manual. Western Psychological Services.

Rutter, M., Le Couteur, A., \& Lord, C. (2003). ADI-R: The Autism Diagnostic InterviewRevised. Los Angeles, CA: Western Psychological Services.

Ryan, J. J. (1981). Clinical utility of a WISC-R short form. Journal of clinical psychology, 37(2), 389-391.

Sattler, J. M. (1992). Assessment of children: WISC-III and WPPSI-R supplement. San Diego: Jerome M. Sattler.

Saxe, R., \& Young, L. (2013). Theory of Mind: How brains think about thoughts. The Oxford Handbook of Cognitive Neuroscience, 2, 204-213. doi:

10.1093/oxfordhb/9780199988709.013.0013 
Schick, B., De Villiers, P., De Villiers, J., \& Hoffmeister, R. (2007). Language and theory of mind: A study of deaf children. Child development, 78(2), 376-396. doi 10.1111/j.1467-8624.2007.01004.x

Shah, A., \& Frith, U. (1993). Why do autistic individuals show superior performance on the block design task? Journal of Child Psychology and Psychiatry, 34, 1351-1364. doi: 10.1111/j.1469-7610.1993.tb02095.x

Shamay-Tsoory, S. G., Harari, H., Aharon-Peretz, J., \& Levkovitz, Y. (2010). The role of the orbitofrontal cortex in affective theory of mind deficits in criminal offenders with psychopathic tendencies. Cortex, 46(5), 668-77. doi: 10.1016/j.cortex.2009.04.008

Shamay-Tsoory, S. G., Shur, S., Barcai-Goodman, L., Medlovich, S., Harari, H., \& Levkovitz, Y. (2007). Dissociation of cognitive from affective components of theory of mind in schizophrenia. Psychiatry Research, 149, 11-23. doi: 10.1016/j.psychres.2005.10.018

Shirama, A., Kato, N., \& Kashino, M. (2017). When do individuals with autism spectrum disorder show superiority in visual search? Autism, 21(8), 942-951. doi: $10.1177 / 1362361316656943$

Shulman, C., Guberman, A., Shiling, N., \& Bauminger, N. (2012). Moral and social reasoning in autism spectrum disorders. Journal of autism and developmental disorders, 42(7), 1364-1376.

Smetana, J. G. (1981). Preschool children's conceptions of moral and social rules. Child development, 1333-1336. doi: 10.2307/1129527

Smetana, J. G. (1983). Social-cognitive development: Domain distinctions and coordinations. Developmental Review, 3(2), 131-147. doi: 10.1016/02732297(83)90027-8

Smetana, J. G. (1985). Preschool children's conceptions of transgressions: Effects of varying moral and conventional domain-related attributes. Developmental Psychology, 21(1), 18. doi: 10.1037/0012-1649.21.1.18

Smetana, J. G. (1989). Toddlers' social interactions in the context of moral and conventional transgressions in the home. Developmental Psychology, 25, 499-508.

Smetana, J. (2006). Social-cognitive domain theory: Consistencies and variations in children's moral and social judgments. In M. Killen \& J. Smetana (Eds.), Handbook of moral development (pp. 119 -153). Mahwah, NJ: Erlbaum. 
Smetana, J. G., \& Braeges, J. L. (1990). The development of toddlers' moral and conventional judgments. Merrill-Palmer Quarterly 36(3), 329-346.

Smetana, J. G., Jambon, M., Conry-Murray, C., \& Sturge-Apple, M. L. (2012). Reciprocal associations between young children's developing moral judgments and theory of mind. Developmental Psychology, 48(4), 1144.

Sotillo, M., \& Rivière, A. (2001). Cuando los niños usan las palabras para engañar: La mentira como instrumento al servicio del desarrollo de las habilidades de inferencia mentalista [When children use words to deceive: Lying as an instrument for developing mentalistic inference abilities]. Infancia y Aprendizaje, 24(3), 291-305. doi: $10.1174 / 021037001316949239$

Sousa, P. (2009). On testing the 'moral law'. Mind \& Language, 24(2), 209-234. doi: $10.1111 / \mathrm{j} .1468-0017.2008 .01360 . \mathrm{x}$

Spek, A. A., Scholte, E. M., \& Van Berckelaer-Onnes, I. A. (2010). Theory of mind in adults with HFA and Asperger syndrome. Journal of Autism and Developmental Disorders, 40(3), 280-289. doi: 10.1007/s10803-009-0860-y

Spence, S. A., Hunter, M. D., Farrow, T. F., Green, R. D., Leung, D. H., Hughes, C. J., \& Ganesan, V. (2004). A cognitive neurobiological account of deception: evidence from functional neuroimaging. Philosophical Transactions of the Royal Society B: Biological Sciences, 359(1451), 1755.

Strauss, E., Sherman, E. M., \& Spreen, O. (2006). A compendium of neuropsychological tests: Administration, norms, and commentary. American Chemical Society.

Stich, S., Fessler, D. M., \& Kelly, D. (2009). On the morality of harm: A response to Sousa, Holbrook and Piazza. Cognition, 113(1), 93-97. doi: 10.1016/j.cognition.2009.06.013

Stone, V. E., Baron-Cohen, S., Calder, A., Keane, J., \& Young, A. (2003). Acquired theory of mind impairments in individuals with bilateral amygdala lesions. Neuropsychologia, 41(2), 209-220. doi: 10.1016/S0028-3932(02)00151-3

Stone, V. E., Baron-Cohen, S., \& Knight, R. T. (1998). Frontal lobe contributions to theory of mind. Journal of Cognitive Neuroscience, 10, 640-656. doi: $10.1162 / 089892998562942$

Surber, C. F. (1977). Development processes in social inference: Averaging of intentions and consequences in moral judgment. Developmental Psychology, 13(6), 654. doi: 10.1037/0012-1649.13.6.654 
Surian, L., \& Leslie, A. M. (1999). Competence and performance in false belief understanding: A comparison of autistic and normal 3-year-old children. British Journal of Developmental Psychology, 17(1), 141-155.

Talwar, V., Zwaigenbaum, L., Goulden, K. J., Manji, S., Loomes, C., \& Rasmussen, C. (2012). Lie-telling behavior in children with autism and its relation to false-belief understanding. Focus on Autism and Other Developmental Disabilities, 27(2), 122129. doi: $10.1177 / 1088357612441828$

Talwar, V., \& Lee, K. (2008). Social and cognitive correlates of children's lying behavior. Child development, 79(4), 866-881. doi: 10.1111/j.1467-8624.2008.01164.x

Thomas, P. (2011). 'Mate crime': ridicule, hostility and targeted attacks against disabled people. Disability \& Society, 26(1), 107-111. doi: 10.1080/09687599.2011.532590

Tomasello, M. (1995). Joint attention as social cognition. In C. Moore and P. Dunham (Eds.), Joint attention: Its origins and role in development, 103130. NY \& London: Psychology Press.

Tomasello, M., Carpenter, M., Call, J., Behne, T., \& Moll, H. (2005). In search of the uniquely human. Behavioral and brain sciences, 28(5), 721-727. doi: $10.1017 / \mathrm{S} 0140525 \mathrm{X} 05540123$

Trope, Y., \& Liberman, N. (2010). Construal-level theory of psychological distance. Psychological review, 117(2), 440. doi: 10.1037/a0018963

Turiel, E. (1978). Social regulations and domains of social concepts. New Directions for Child and Adolescent Development, 1978(1), 45-74.

Turiel, E. (1983). The development of social knowledge: Morality and convention. Cambridge University Press.

Turiel, E. (2006). Thought, emotions, and social interactional processes in moral development. In M. Killen and J. Smetana (Eds.), Handbook of moral development (pp. 25-54). Psychology Press.

Wainryb, C., Brehl, B. A., Matwin, S., Sokol, B. W., \& Hammond, S. (2005). Being hurt and hurting others: Children's narrative accounts and moral judgments of their own interpersonal conflicts. Monographs of the society for research in child development, i-122. 
Walsh, C. R., \& Byrne, R. M. (2007). How people think “if only...” about reasons for actions. Thinking \& Reasoning, 13(4), 461-483.

Wang, Y., \& Su, Y. (2006). Theory of mind in old adults: The performance on Happé's stories and faux pas stories. Psychologia, 49(4), 228-237.

Wechsler, D. (1991). WISC-III: Wechsler Intelligence Scale for Children - Third Edition. San Antonio, TX: Psychological Corporation.

Wechsler, D. (2005). WISC-IV: Wechsler intelligence scale for children, 4th Edition. Madrid: TEA Ediciones.

Weiner, B. (1995). Judgments of responsibility: A foundation for a theory of social conduct. Guilford Press.

Weisberg, D. S., and Leslie, A. M. (2012). The role of victims' emotions in preschoolers' moral judgments. Rev. Philos. Psychol. 3, 439-455. doi: 10.1007/s13164-012-0101-8

Wellman, H. M., Cross, D., \& Watson, J. (2001). Meta-analysis of theory-of-mind development: The truth about false belief. Child development, 72(3), 655-684.

Wellman, H. M., \& Lagattuta, K. H. (2000). Developing understandings of mind. En S. Baron-Cohen, H. Tager-Flusberg \& D. J. Cohen (Eds.), Understanding other minds: Perspectives from developmental cognitive neuroscience (2nd ed.) 21-49. Oxford, UK: Oxford University Press.

Wellman, H. M., \& Liu, D. (2004). Scaling of theory-of-mind tasks. Child development, 75(2), 523-541. doi: 10.1111/j.1467-8624.2004.00691.x

Wimmer, H., \& Perner, J. (1983). Beliefs about beliefs: Representation and constraining function of wrong beliefs in young children's understanding of deception. Cognition, 13(1), 103-128. doi: 10.1016/0010-0277(83)90004-5

Wiseman, R. (1995). The megalab truth test. Nature, 373(6513), 391.

Young, L., Cushman, F., Hauser, M., \& Saxe, R. (2007). The neural basis of the interaction between theory of mind and moral judgment. Proceedings of the National Academy of Sciences, 104(20), 8235-8240. doi: 10.1073/Pnas.0701408104.

Young, L., \& Saxe, R. (2009). Innocent intentions: A correlation between forgiveness for accidental harm and neural activity. Neuropsychologia, 47, 2065-2072. doi: 10.1016/j.neuropsychologia.2009.03.020. 
Young, L., \& Tsoi, L. (2013). When mental states matter, when they don't, and what that means for morality. Social and Personality Psychology Compass, 7(8), 585-604. doi: $10.1111 / \mathrm{spc} 3.12044$

Yuill, N., \& Perner, J. (1988). Intentionality and knowledge in children's judgments of actor's responsibility and recipient's emotional reaction. Developmental Psychology, 24(3), 358. doi: 10.1037/0012-1649.24.3.358

Yuill, N. (1984). Young children's coordination of motive and outcome in judgements of satisfaction and morality. British Journal of Developmental Psychology, 2(1), 73-81. doi: 10.1111/j.2044-835X.1984.tb00536.x

Zalla, T., Barlassina, L., Buon, M., \& Leboyer, M. (2011). Moral judgment in adults with autism spectrum disorders. Cognition 121, 115-126. doi:

10.1016/j.cognition.2011.06.004

Zalla, T., \& Leboyer, M. (2011). Judgment of intentionality and moral evaluation in individuals with high functioning autism. Review of Philosophy and Psychology, 2(4), 681-698. doi: 10.1016/j.cognition.2011.06.004

Zalla, T., Sav, A. M., Stopin, A., Ahade, S., \& Leboyer, M. (2009). Faux pas detection and intentional action in Asperger Syndrome. A replication on a French sample. Journal of Autism and Developmental Disorders, 39(2), 373-382. doi: 10.1007/s10803-0080634-y

Zelazo, P.D, Helwig, C.C, \& Lau, A. (1996). Intention, act, and outcome in behavioral prediction and moral judgment. Child Development, 67, 2478-2492.

doi: $10.2307 / 1131635$ 
Capítulo 7 Apéndices / Appendix 


\subsection{Apéndice 0. Permisos para la administración de las tareas (TEA y NT)}

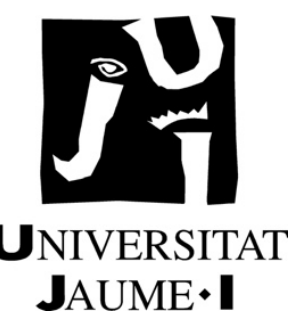

A LA ATENCIÓN DE:

Padre/Madre de:

C. P.

Los miembros del Servicio de Atención Psicológica Infantil (S.A.P.I) del Departamento de Psicología Evolutiva y de la Educación, de la Universidad Jaime I: solicitamos su permiso para poder examinar a su hijo/a diagnosticado de Asperger o TEA nivel 1, el próximo curso 2015-2016.

Las sesiones con su hijo/a serían individuales, con un tiempo estimado de 45 minutos repartidos en tres sesiones.

Este estudio se inscribe dentro de una investigación para una tesis doctoral, y cuenta con el permiso de la Dirección Territorial de Cultura, Educación y Deporte, de la Universitat Jaume I y del Centro. Nuestro trabajo, por tanto, está sujeto a los cánones éticos y morales propios de un trabajo de este tipo. En este sentido, estamos a su disposición (e-mail de contacto) para explicarles cualquier cuestión que suscite su interés. Reciba un cordial saludo.

Castellón, de de 20

Dra. Rosa Ana Clemente, Coordinadora del Proyecto de Investigación

$\mathrm{PhD}$ Irene Garcia Molina e-mail de contacto: imolina@uji.es

\section{(Devolver a la Universidad firmado)}

Doy mi autorización para que el alumno:

Del curso:

SI PARTICIPE EN ESTE ESTUDIO

(FIRMA)

Padre / Madre / Tutor 


$$
\text { C. P. }
$$

Los miembros del Servicio de Atención Psicológica Infantil (S.A.P.I) del Departamento de Psicología Evolutiva y de la Educación, de la Universidad Jaime I: solicitamos su permiso para poder examinar a su hijo/a, por pertenecer al grupo clase de un compañero diagnosticado de Asperger o TEA nivel 1, el próximo curso 2015-2016. Las tareas comprenderán actividades relacionadas con habilidades sociales y cognitivas. Posteriormente, se les adjuntará un informe con las puntuaciones más destacadas del análisis de los datos. Las sesiones con su hijo/a serían individuales, con un tiempo estimado de 45 minutos repartidos en tres sesiones.

Este estudio se inscribe dentro de una investigación para una tesis doctoral, y cuenta con el permiso de la Dirección Territorial de Cultura, Educación y Deporte, y de la Universidad Jaume I, así como del Centro Escolar. Nuestro trabajo, por tanto, está sujeto a los cánones éticos y morales propios de un trabajo de este tipo. En este sentido, estamos a su disposición (e-mail de contacto) para explicarles cualquier cuestión que suscite su interés. Reciba un cordial saludo.

Castellón, de de 20

Dra. Rosa Ana Clemente, Coordinadora del Proyecto de Investigación

$\mathrm{PhD}$ Irene Garcia Molina e-mail de contacto: imolina@uji.es

(Devolver firmado)

Doy mi autorización para que el alumno:

Del curso:

SI PARTICIPE EN ESTE ESTUDIO

(FIRMA)

Padre / Madre / Tutor 


\subsection{Apéndice 1. Historias visuales de metedura de pata / Visual Faux Pas stories}

De Garcia-Molina et al. (2016) / From Garcia-Molina et al. (2016).

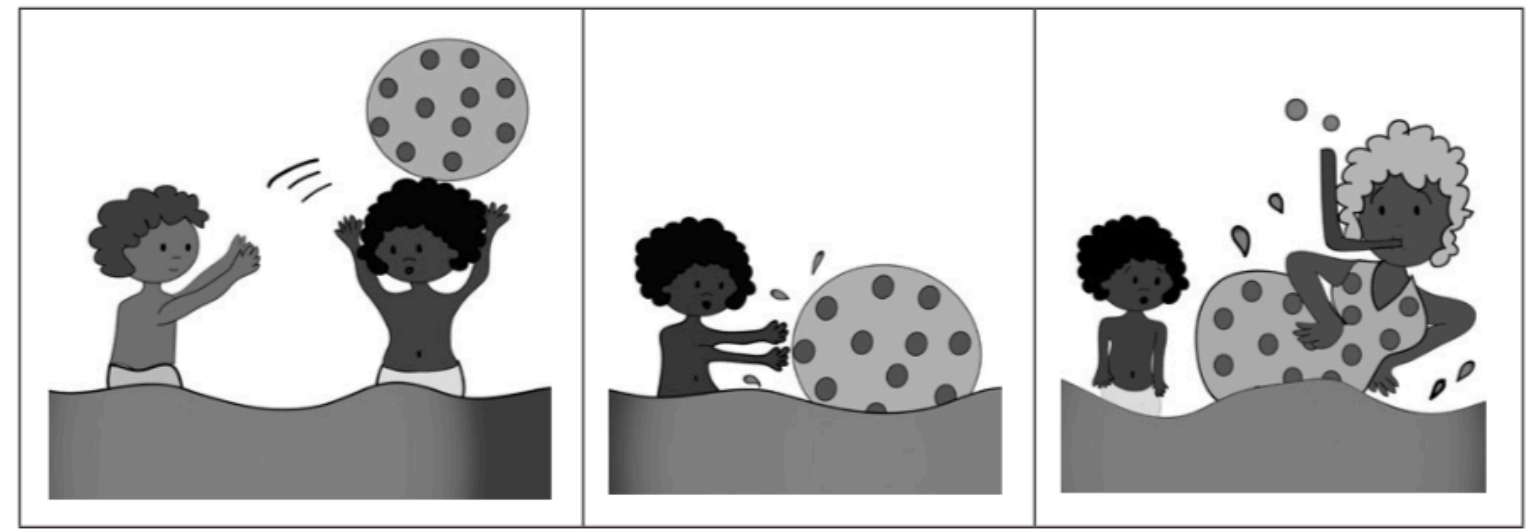

Figure 7-1. Historia de la pelota / The ball story

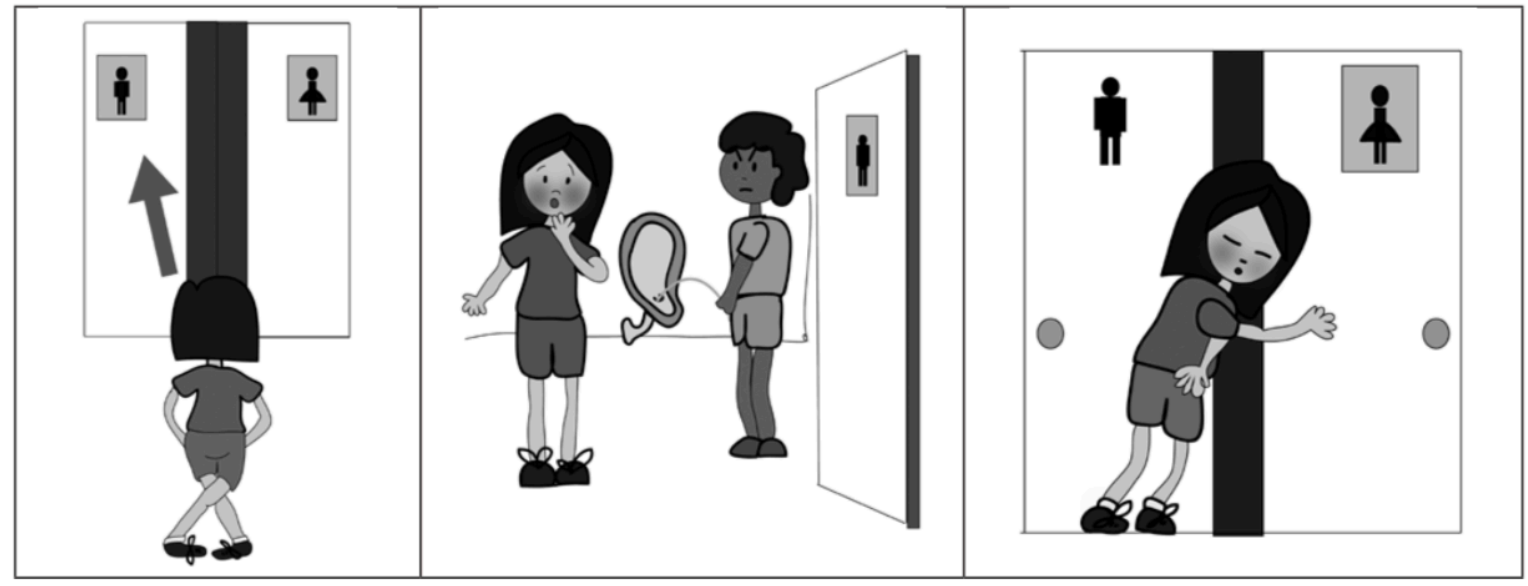

Figure 7-2. Historia del baño / The WC story

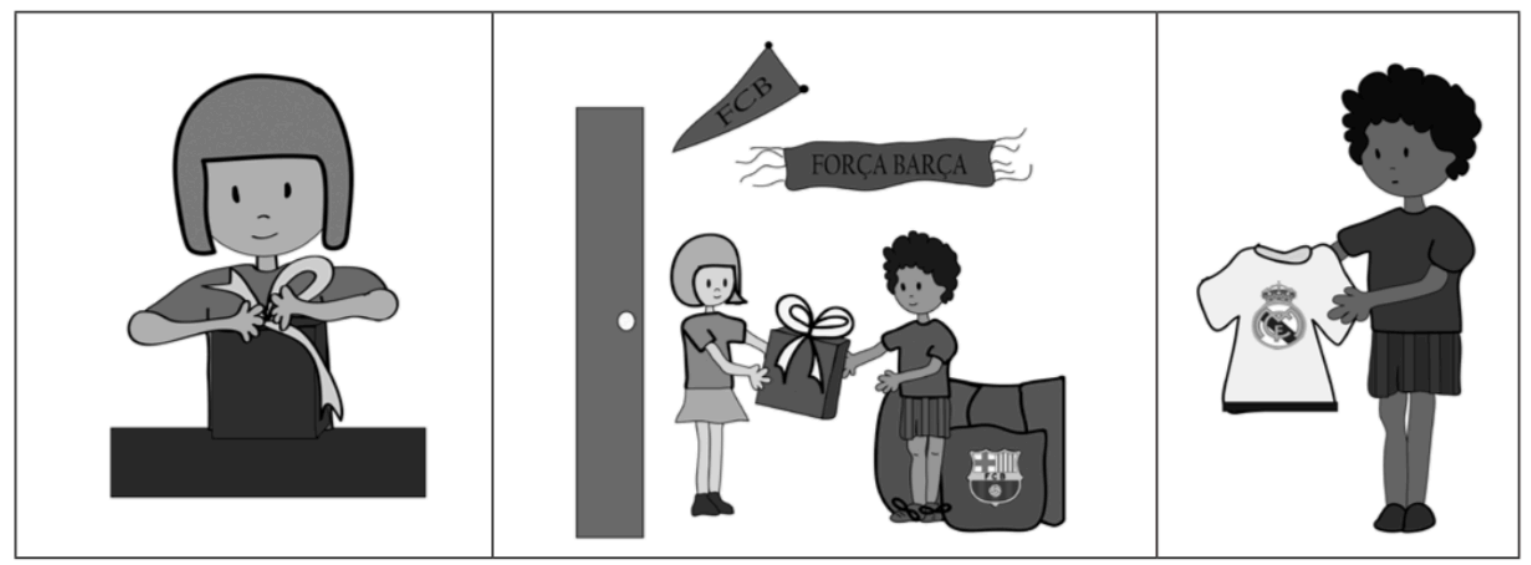

Figure 7-3. Historia del regalo / The present story 


\subsection{Apéndice 2. Historias verbales de metedura de pata / Verbal Faux Pas stories}

“Cumpleaños". Era el cumpleaños de Olga, y su novio Joan, le estaba organizando una fiesta sorpresa. Ana estaba ayudándole. Entonces, Joan le recordó a Ana: (Voz de Joan) «Y recuerda Ana, que Olga no debe saber nada de lo de su fiesta de cumpleaños sorpresa para mañana». «Sí, sí, Joan. No te preocupes». Unas horas más tarde... Voz de Ana: «Hola Olga, ¿cómo estás?... Bueno, tengo prisa. ¡Nos vemos mañana!»Voz de Olga: - «¿Mañana?». Voz de Ana: - «Sí, en tu fiesta de cumple. Nada, te dejo que voy muy apurada».

"Birthday". Olga's boyfriend, Joan, was having a surprise party for Olga's birthday. Joan invited her friend Ana. Ana was helping him. Then, Joan said: (Voice of Joan) «Ana, remember that Olga should not know anything about her surprise party for tomorrow». «Yes, yes, Joan. Do not worry". A few hours later ... Voice of Ana: «Hello Olga, how are you? ... Well, I'm in a hurry. See you tomorrow! » Voz de Olga: - «Tomorrow? ». Voz de Ana: - "Yes, at your party. Well, I have to go!».

"Examen". Se encontraron en el patio de la escuela Lorenzo y Marta, y Lorenzo le preguntó a Marta: «Hola Marta, ¿qué tal el examen?». Voz de Marta: «Fatal, he sacado un 3 sobre 10». Voz de Lorenzo: «Ay, pobre... Mira, ahí está Helena, vamos a preguntarle... Hola Helena, ¿qué tal tu examen?»Voz de Helena: «Buah, genial, un 10 ¡es que tienes que ser muy tonto para no aprobar un examen tan fácil!».

“Exam". Lorenzo and Marta met in the school, and Lorenzo asked Marta: «Hi Marta, how was the exam? » Voice of Marta: «Very bad, I've got a 3 out of 10». Lorenzo's voice: «Oh, poor Marta... Look, there's Helena, let's ask her ... Hello Helena, how's your exam?» Voice of Helena: «Buah, great, excellent, 10! You have to be very stupid to not pass such an easy test!» 
"Melena". En la cola del supermercado había dos chicos mirando a la persona que estaba delante. Esa persona era muy alta y tenía una melena larguísima. Un amigo, le dijo al otro: «Llevo diez minutos mirándola. Mírala, está ahí, ¿la ves? ¡Qué melena tan larga, qué piernas tan esbeltas! Es altísima. Uf, me acabo de enamorar, tengo que ir a hablar con ella. Es la mujer de mi vida». Unos segundos más tarde... «Disculpa, guapa...»Voz de hombre muy grave: «¿Sí? ¿Querías algo?».

"Hair". In the supermarket queue there were two guys looking at the person who was in front of them. That person was very tall and had very long hair. A friend told the other: «I've been watching her for ten minutes. Look at her, she is there, can you see her? What a long hair, what slender legs! She is very tall. I just fell in love with her, I have to talk to her. She is the woman of my life ». A few seconds later ... «Excuse me, beautiful ...»Very serious man's voice: «Sorry? Did you want something?» 


\subsection{Apéndice 3. Historias mixtas de metedura de pata / Mixed Faux Pas stories}

De Baron-Cohen et al. (1999) / From Baron-Cohen et al. (1999).

“Pastel de manzana”. Inés ayudó a su mamá a hacer un pastel de manzana para su tío, que vino a visitarlas. Ella lo llevó hasta su tío y le dijo: «Lo hice solo para ti». «Mmm», se le ve buena pinta... — respondió el tío Tomás, «Me encantan los pasteles, el único que no me gusta nada, nada, ¡es el de manzana!».

“Apple pie". Inés helped her mum make an apple pie for her uncle when he came to visit. She carried it out of the kitchen. «I made it just for you», said Inés. «Mmm», replied Uncle Tomás, «That looks lovely. I really love pies, except for apple, of course! »

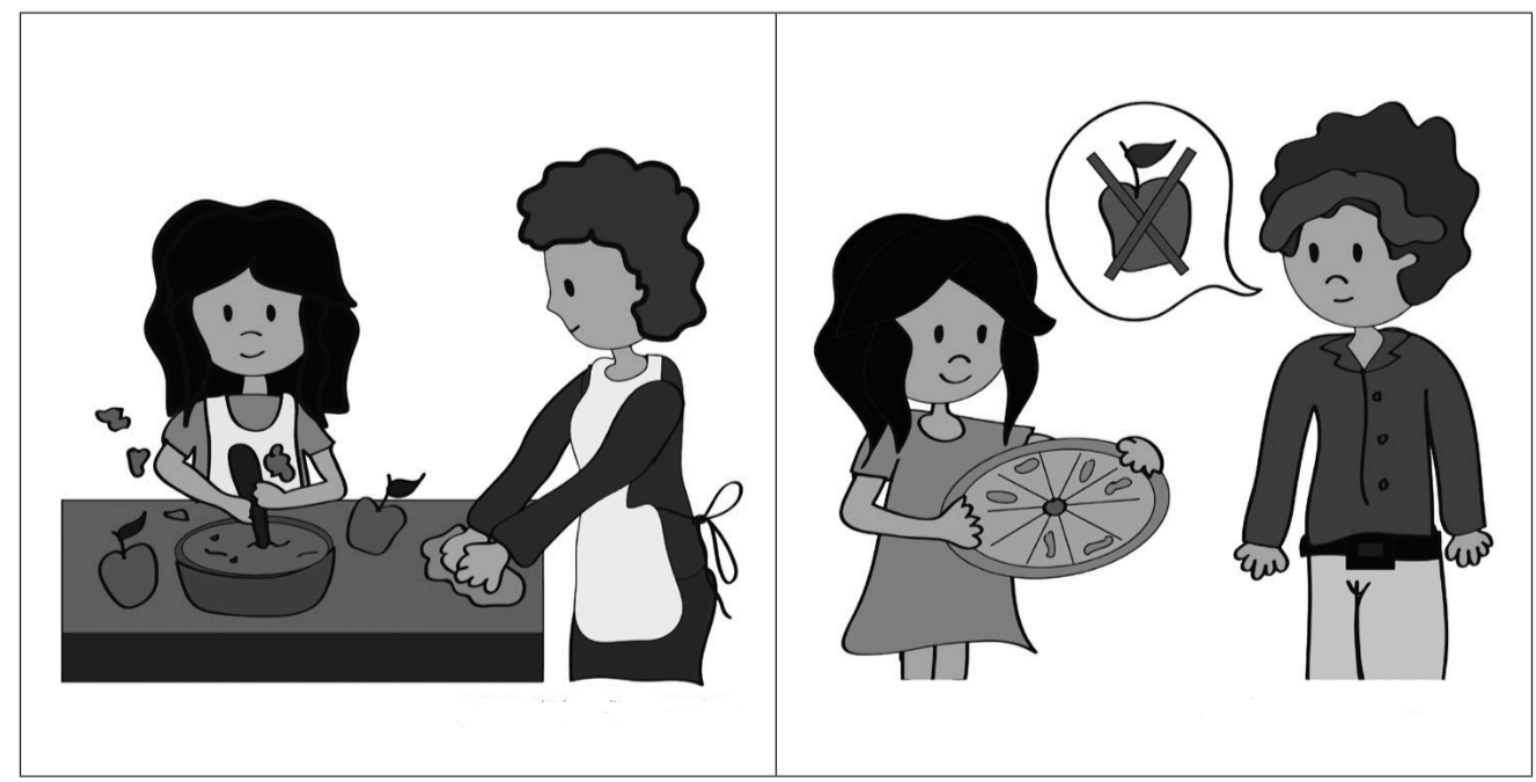

Figure 7-4. Historia del pastel de manzana / Apple pie story

“EI avión”. Ismael le compró a Judit un avión de juguete para su cumpleaños. Unos meses más tarde, estaban jugando juntos, cuando accidentalmente Ismael dejó caer el avión y rompió un ala. «No te preocupes», dijo Judit, «De todos modos, nunca me gustó. Alguien me lo regaló por mi cumpleaños». 
"The plane". Ismael bought Judit a toy airplane for her birthday. A few months later, they were playing together with that same plane, and Ismael accidentally dropped it. Judit said to him: «Do not worry, Ismael. I never liked it anyway. Somebody gave it to me for my birthday».

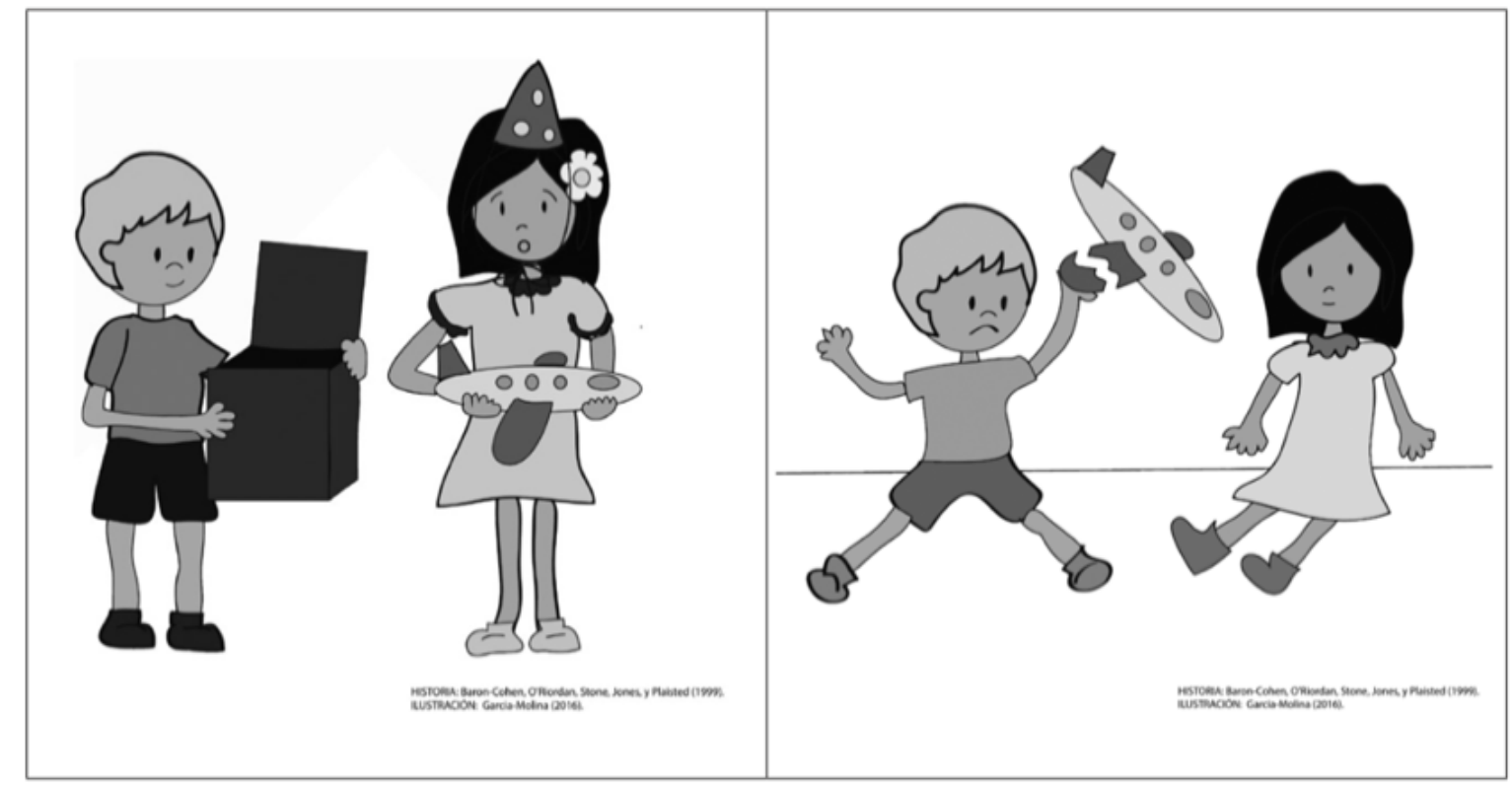

Figure 7-5. Historia del avión / The plane story

“Cubículos”. Irene estaba en uno de los cubículos de los baños de la escuela. Teresa y Lara estaban en los lavabos cercanos. Teresa dijo: «¿Conoces a la chica nueva de clase?». «Sí, se llama Irene. La verdad, parece algo extraña»—respondió Lara. Entonces, Irene, salió de los cubículos. Y Lara dijo: «¡Oh!, hola, Irene, ¿vienes a jugar con nosotras?».

"Cubicles". Irene was in one of the cubicles in the toilets at school. Teresa and Lara were at the sinks nearby. Teresa said «Do you know that new girl in the class?», «Yes, her name is Irene. Doesn't she look really weird!» — replied Lara. Irene then came out of the cubicles. Teresa said «Oh, hello Irene, do you want to come and play rounders?». 


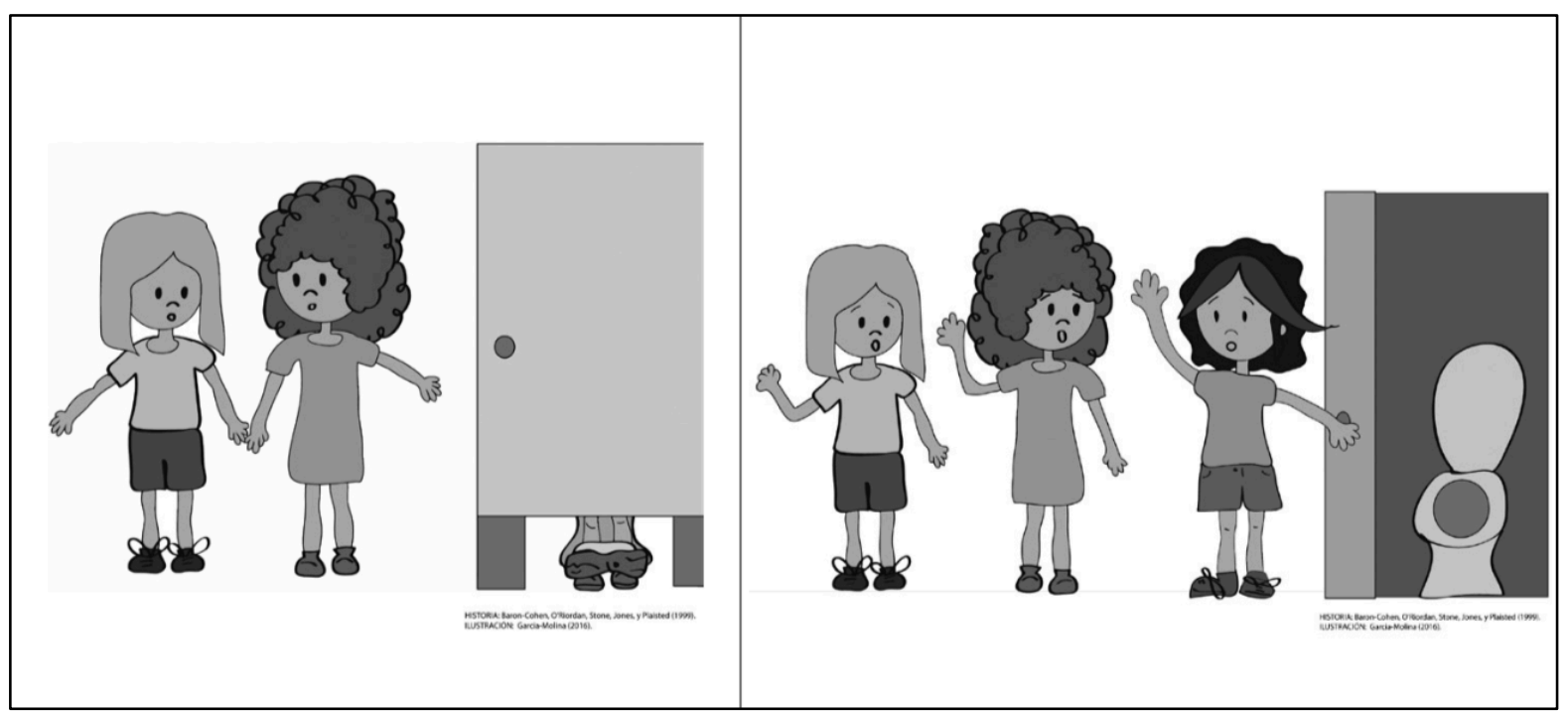

Figure 7-6. Historia de los cubículos / Cubicles story

“Cocinera". Laia era nueva en la escuela. Le dijo a su nuevo amigo Daniel: «Mi mamá es la cocinera del comedor en esta escuela». Entonces Clara se acercó a ellos y dijo: «Uf, odio la comida del comedor, y a la cocinera, ¡es horrible!».

"Dinner lady". Laia had just started at a new school. He said to her new friend, Daniel, «My Mum is a dinner lady at this school». Then Clara came over and said, «I hate dinner ladies. They're horrible!».

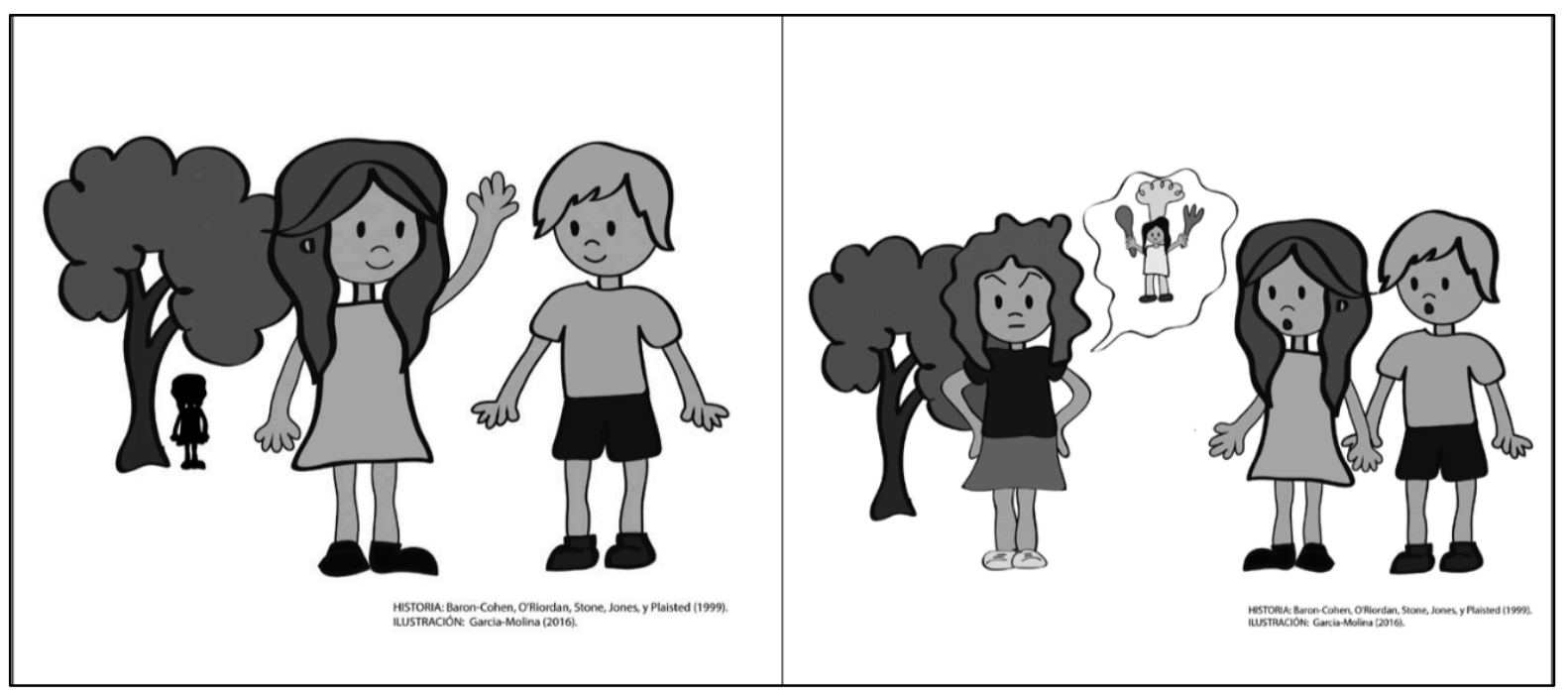

Figure 7-7. Historia de la cocinera / Dinner lady story 


\title{
7.4 Apéndice 4. Historias verbales morales / Verbal moral stories.
}

\author{
Del Estudio 1 / From Study 1
}

Dog story. Child: «Well, Bobby, we are going to go to the park, but before, let me eat a little bit of this fabulous chocolate cake!» (The dog barks. Door opening sound). Mommy: «Gee! Who ate the whole cake that I prepared for tonight's party?» Child: «Mmm... It was Bobby, mommy. Bobby ate all the cake! » Mommy: «Naughty dog, Bobby, naughty dog! Tonight, you are not going to sleep in the house!» (The dog cries. Raining sound.)

Car story. (Car sound). Melissa: «Oh, no... Oliver! I left my keys inside the car, and we are late... Now, what could I do, Oliver? » Oliver: «Wait a moment, Melissa!» (Glass crashing. Car alarm very loud) Oliver: «Here you have the keys» Melissa: «But... Oliver, what did you do!?»

Sandwich story. Fred: «Hello, Dominique! Wow, that sandwich looks great! And how hungry I am... Dominique: «Do you want a bit? Take a bite! I am going for a drink and I'll come back. (Someone's eating noise). Dominique: «But, but, Fred! I told you a bite! You ate the whole sandwich!» 


\subsection{Apéndice 5. Historias mixtas morales / Mixed moral stories}

De Garcia-Molina et al. (2016) / From Garcia-Molina et al. (2016)

“Yo-yo". Este es Luis y esta es Iris. Luis tiene un yoyo y está jugando con él porque es la hora del patio. Iris va a la clase de al lado, y se lo pide para jugar un poco. Suena el timbre, y todos los niños tienen que entrar en clase. Y Luis también. Pero Iris se esconde el yoyo en su mochila para jugar más tarde en su casa.

"Yo-yo". This is Luis and this is Iris. Luis has a yo-yo and he is playing with it because it is playtime. Iris is his classmate, and asks him to play a little. The bell rings, and all children have to go. Also, Luis. But Iris hides the yo-yo in her school bag to play later at home.

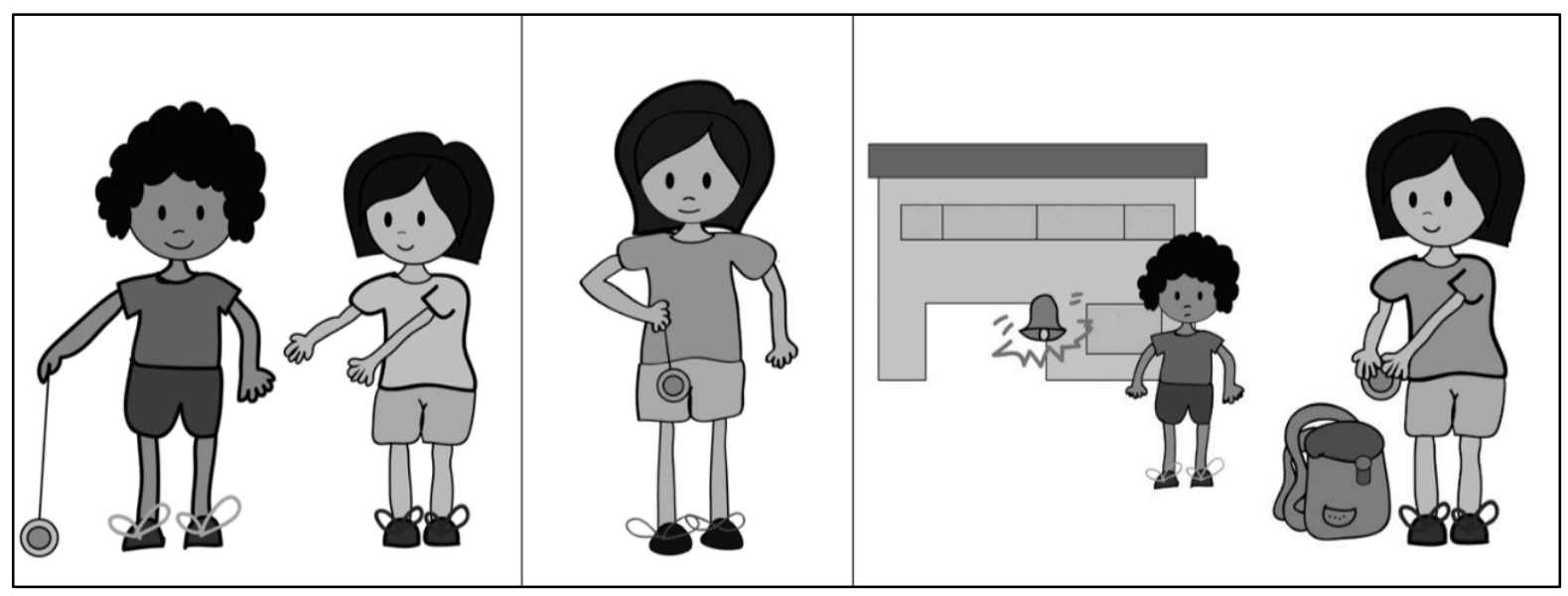

Figure 7-8. Historia del yo-yo / Yo-yo story

“Cola”. Estas niñas están en una fiesta de cumpleaños. Estas niñas son Júlia, Neus y Paula. Es la hora de repartir los helados, y las niñas se ponen en fila. Cuando van a darle el helado a Júlia, Neus se cuela, la aparta y coge el helado. Y Neus se va contenta a comerse su helado.

“Queue”. These girls are at a birthday party. They are Júlia, Neus and Paula. It's time to pick up an ice cream, and the girls line up. When it is the Júlia's turn, Neus jumps the queue, moves Júlia away, and takes the ice cream. Neus is happy to eat her ice cream. 


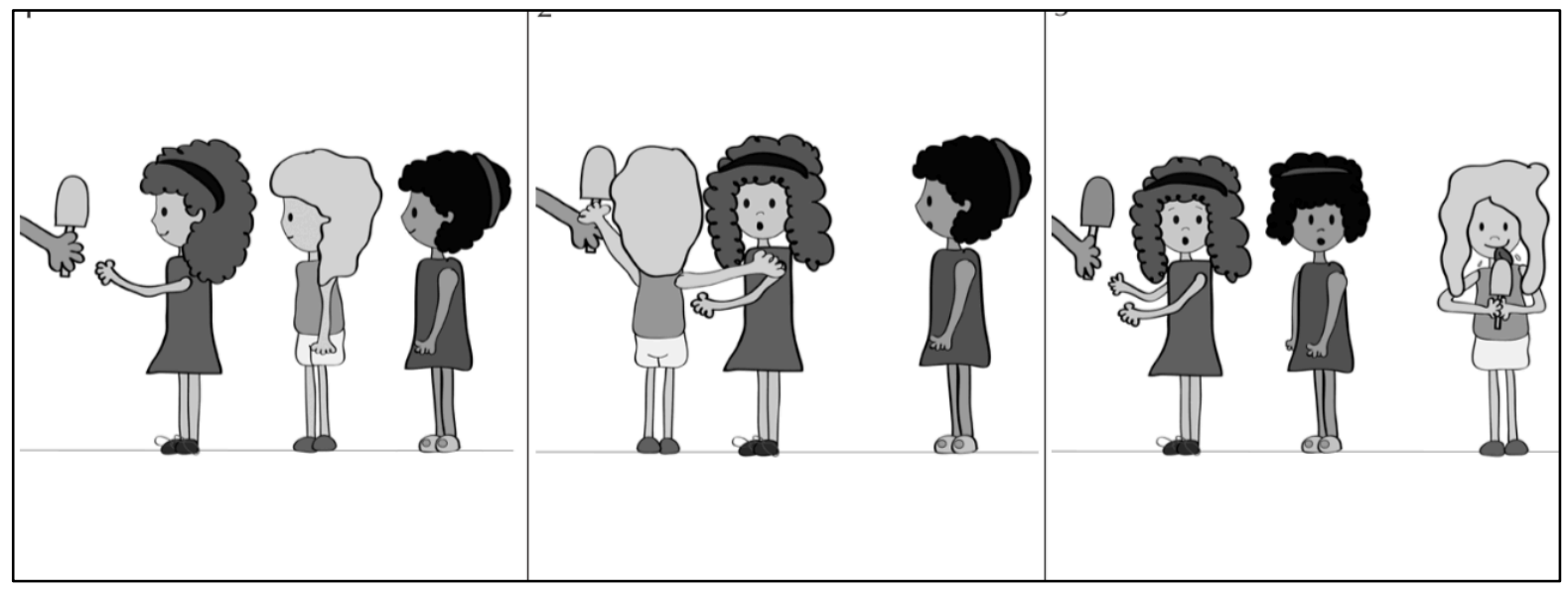

Figure 7-9. Historia de la cola / Queue story

“Globos". Es el cumpleaños de Lola y Adrián, pero toda la clase ha decidido ir al cumple de Lola, y Adrián decide hacer su fiesta otro día. Adrián llega el primero a casa de Lola, y Lola le pide ayuda para colgar los globos. Cuando ya están todos colgados, Lola se va a por el pastel, y Adrián coge una aguja y estalla todos los globos.

"Balloons". It's Lola and Adrián's birthday, but the whole class has decided to go to Lola's birthday, and Adrián decides to have his party another day. Adrián arrives first at Lola's house, and Lola asks him for help to hang the balloons. When the task is done, Lola goes for the cake, and Adrián takes a needle and explodes all the balloons.

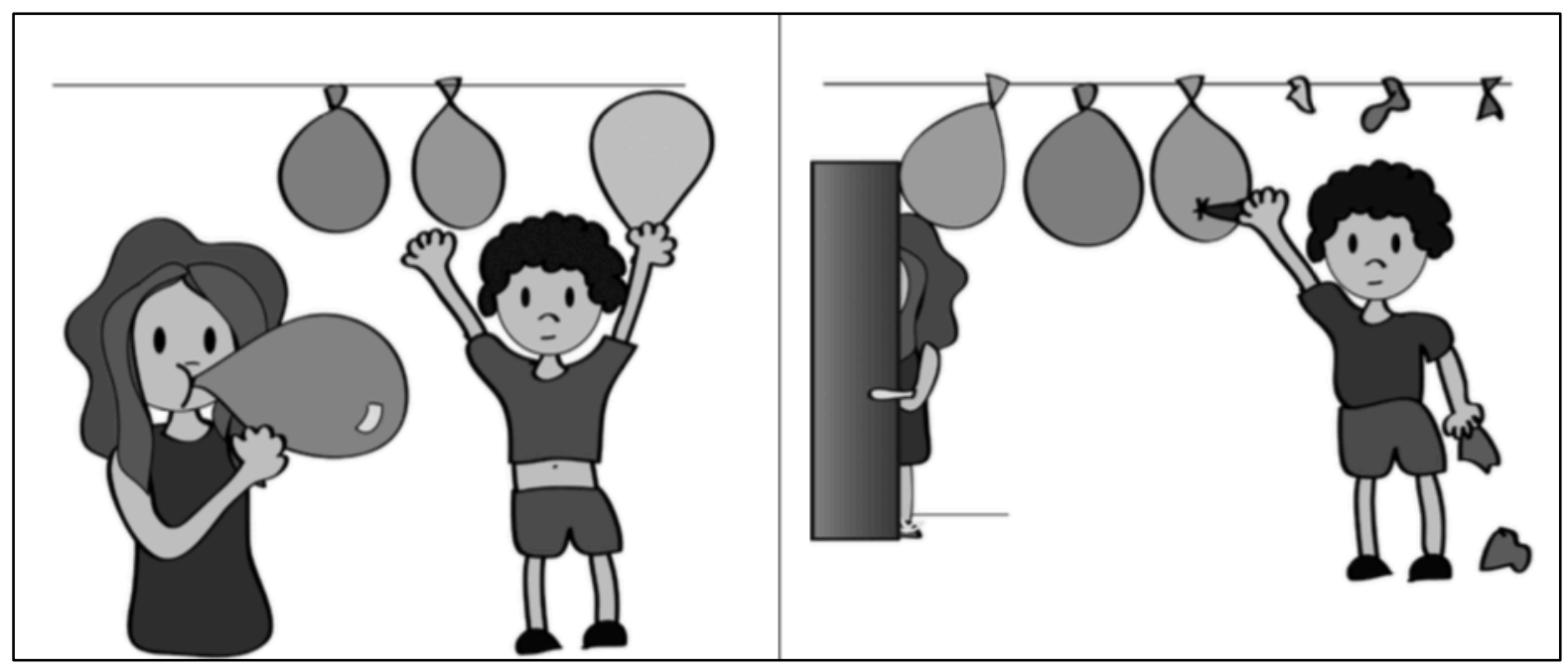

Figure 7-10. Historia de los globos / Ballons story 
“Sopa". Este es Diego, que quiere ver sus dibujos favoritos, pero su madre tiene que irse al médico y le pide que le preparare una sopa a su hermanita Luna. Así que, Diego cocina la sopa. A Luna le encanta la sopa, y quiere probarla enseguida. Diego ve como coge la cuchara, pero se calla y no le avisa de que la sopa aun quema, y su hermanita se quema la lengua.

"Soup". This is Diego, who wants to watch to his favorite cartoons, but his mother has to go to the doctor and asks him to prepare a soup for his little sister Luna. So, Diego cooks the soup. Luna loves soup and she wants to try it right away. Diego sees how Luna takes the spoon, but he keeps quiet and does not let her know that the soup is still burning, and his little sister burns her tongue.

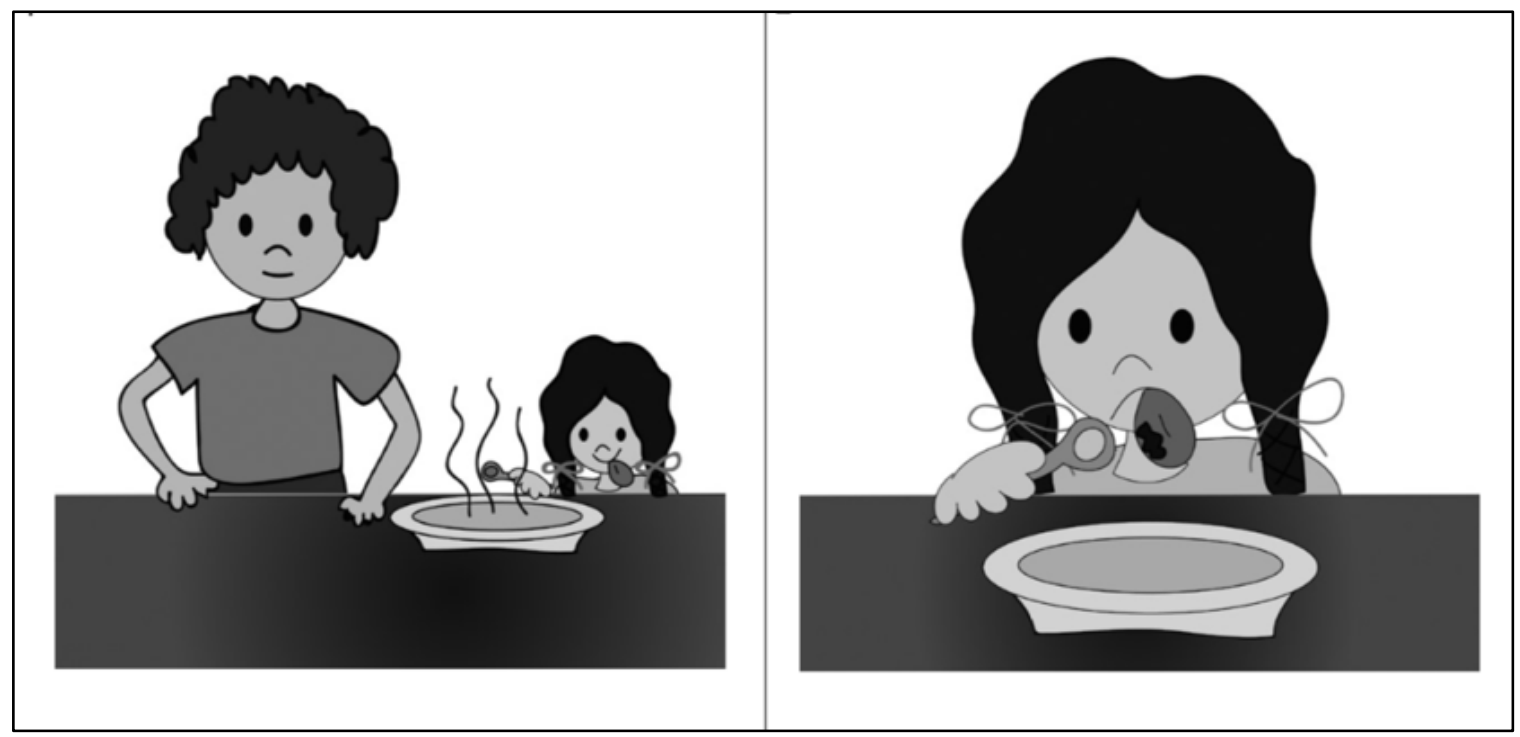

Figure 7-11. Historia de la sopa / Soup story 


\subsection{Apéndice 6. Historia de Falsa Creencia no verbal / Non-verbal False Belief story.}

Tarea de Falsa Creencia de primer orden de contenido inesperado (no-verbal). Basada en las viñetas de De Villiers y De Villiers (2012), extraída de Schick et al. (2007) / First order falsebelief task of unexpected content (non-verbal). It was based on De Villiers and De Villiers (2012) vignettes, from Schick et al. (2007).

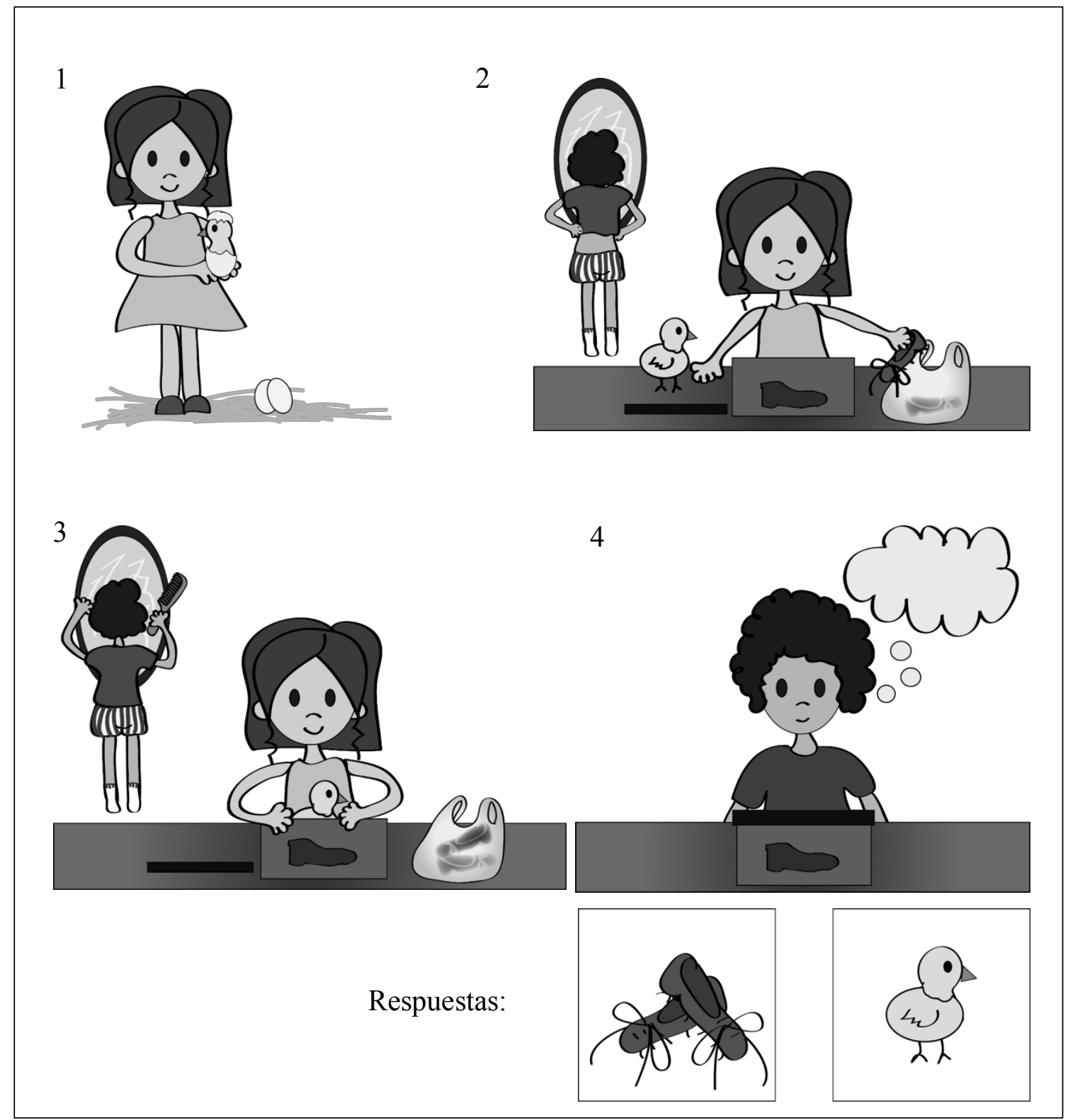

Figura 7-12. Historia de Falsa Creencia no-verbal / Non-verbal False Belief story 